
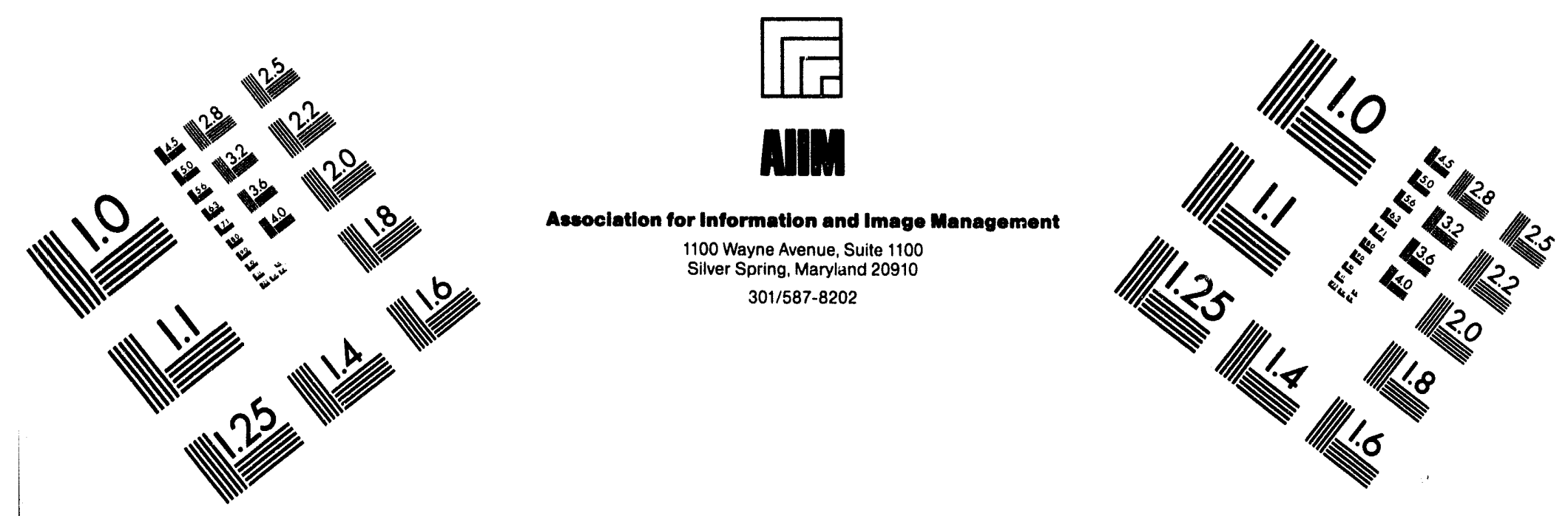

Centimeter

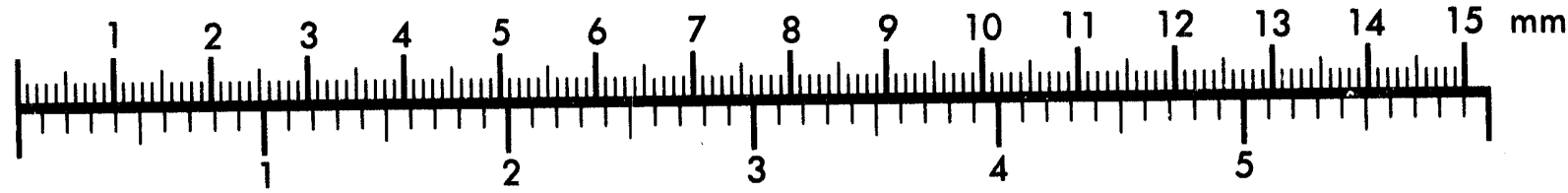

Inches
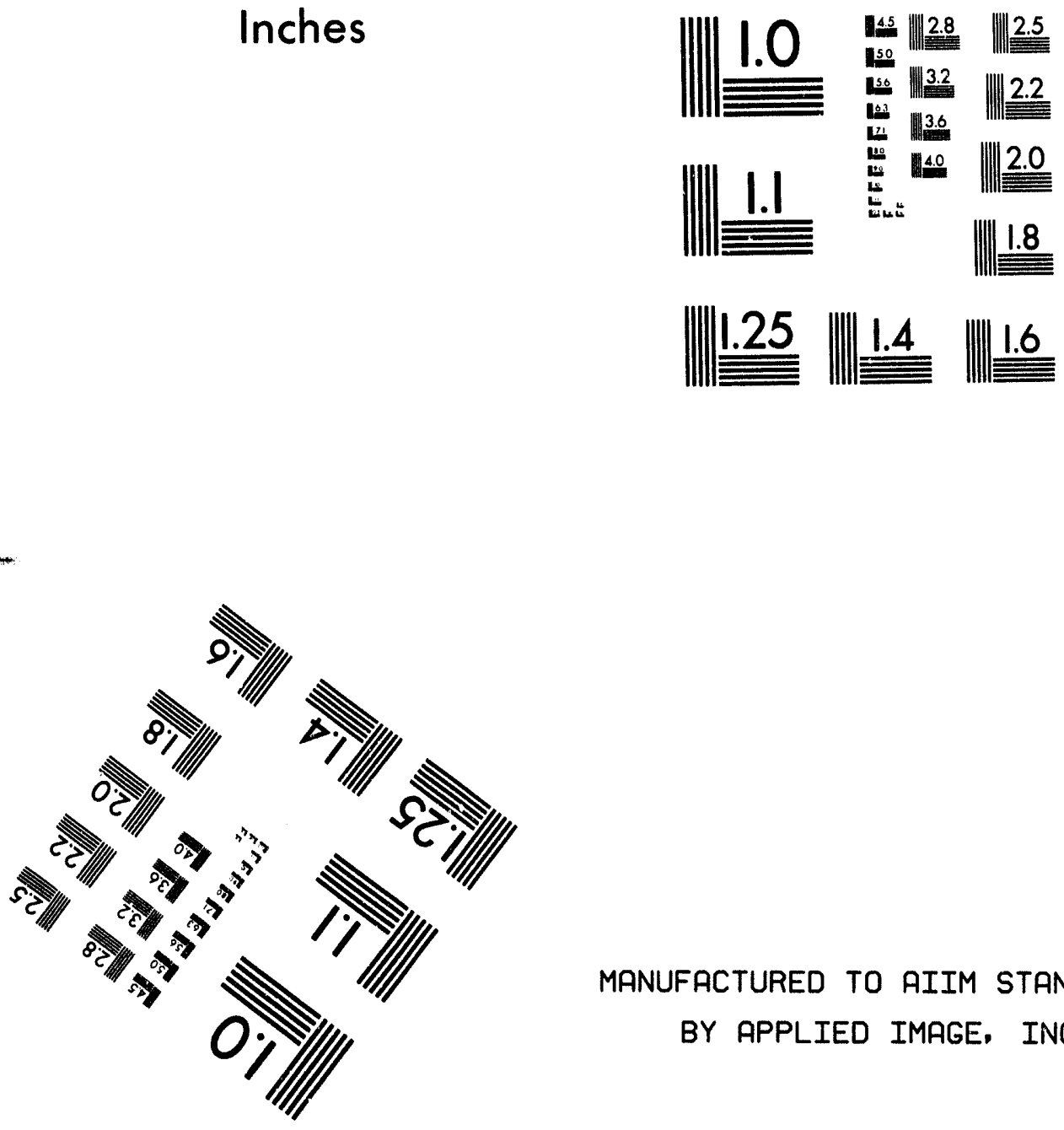

MANUFACTURED TO AIIM STANDARDS

BY APPLIED IMAGE, INC.

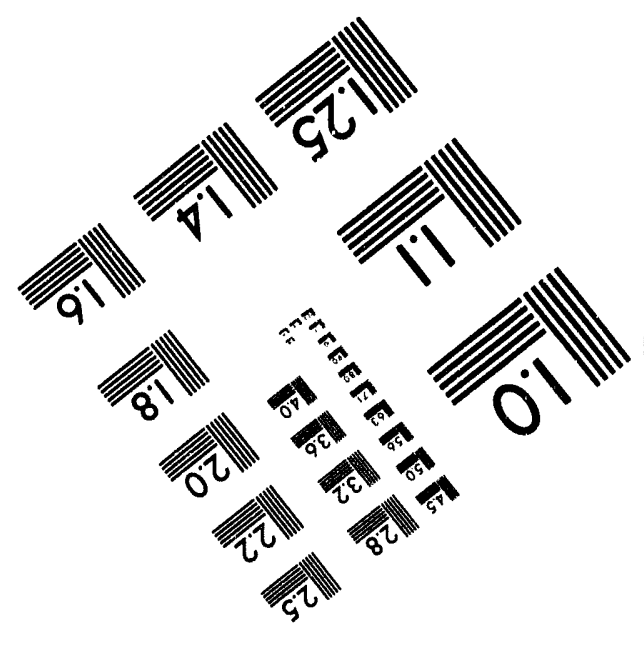



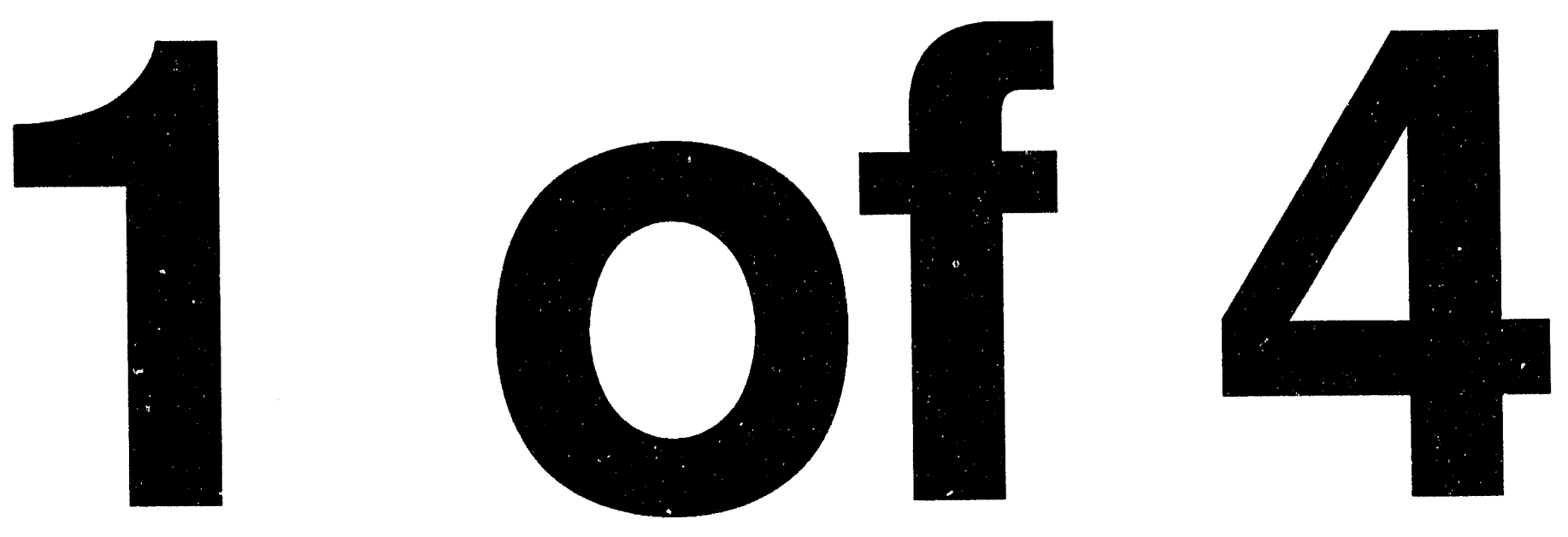
Decontamination Systems Information and Research Program

\section{RECEIVED JUL 181994 OSTI}

\section{Quarterly Report \\ October - December 1993}

February 1994

Work Performed Under Contract No.: DE-FC21-92MC29467

U.S. Department of Energy Office of Environmental Management Office of Technology Development Washington, DC
For

U.S. Department of Energy Office of Fossil Energy

Morgantown Energy Technology Center Morgantown, West Virginia

\section{By}

West Virginia University

National Research Center for Coal and Energy Morgantown, West Virginia 


\section{DISCLAIMER}

This report was prepared as an account of work sponsored by an agency of the United States Government. Neither the United States Govemment nor any agency thereof, nor any of their employees, makes any warranty, express or implied, or assumes any legal liability or responsibility for the accuracy, completeness, or usefulness of any information, apparatus, product, or process disclosed, or represents that its use would not infringe privately owned rights. Reference herein to any specific conmercial product, process, or service by trade name, trademark, manufacturer, or otherwise does not necessarily constitute or imply its endorsement, recommendation, or favoring by the United States Government or any agency thereof. The views and opinions of authors expressed herein do not necessarily state or reflect those of the United States Government or any agency thereof.

This report has been reproduced directly from the best available copy.

Available to DOE and DOE contractors from the Office of Scientific and Technical Information, 175 Oak Ridge Tumpike, Oak Ridge, TN 37831; prices available at (6J5) 576-8401.

Available to the public from the National Technical Infomation Service, U.S. Department of Commerce, 5285 Port Royal Road, Springfield, VA 22161; whothe orders accepted at (703) 487-4650. 


\title{
Decontamination Systems Information and Research Program
}

\author{
Quarterly Report \\ October - December 1993
}

\section{Work Performed Under Contract No.: DE-FC21-92MC29467}

\section{U.S. Department of Energy Office of Environmental Management Office of Technology Development 1000 Independence Avenue Washington, DC 20585}

For

U.S. Department of Energy

Office of Fossil Energy Morgantown Energy Technology Center P.O. Box 880 Morgantown, West Virginia 26507.0880

By

W «st Virginia University

National Research Center for Coal and Energy

P. O. Box 6064

Morgantown, West Virginia 26506

February 1994 


\section{ADMINISTRATIVE SUMMARY}

West Virginia University (WVU) and the U. S. DOE Morgantown Energy Technology Center (METC) entered into a Cooperative Agreement on August 29, 1992 entitled "Decontamination Systems Information and Research Programs" (DOE Instrument No. : DEFC21-92MC29467). Stipulated within the Agreement is the requirement that WVU submit to METC a series of Technical Progress Reports on a quarterly basis. This report comprises the third Quarterly Technical Progress Report for Year 1 of the Agreement. This report reflects the progress and/or efforts performed on the sixteen (16) technical projects encompassed by the Year 1 Agreement for the period of October 1 through December 31, 1993.

\section{A. Administrative Action}

A Cooperative Agreement was established between WVU and METC in the Fall of 1992. The original Annual Research Plan consisted of six (6) WVU Research projects. NEPA approval for these projects were received by WVU in Mid-March 1993. At the request of METC a presentation, of these six projects, was scheduled for late May 1993, at which time comments and criticisms on these projects were noted.

On June 29, 1993, proposed Projects for the 1994 Annual Plan were submitted to METC. These projects were evaluated for suitability and approved for development. They are included in this quarter.

\section{B. BROIECT VARIANCES, ACCOMPUSHMINTS AND PROBLEMS}

Experimental work has commenced upon receipt of the NEPA approval. However, projects involving laboratory experimentation may have some delay in meeting their time lines due to alignment of work force, equipment and supply procurements. 


\section{A Systematic Assessment of the State of Hazardous Waste Clean-up Technologies \\ (Project MC-1)}

Quarterly Report for Period

for Period October 1 through December 31, 1993

\section{Work Performed Under Contract \\ No.: DE-FC21-92MC29467}

\section{For}

U.S. Department of Energy

Office of Fossil Energy

Morgantown Energy Technology Center

Morgantown, West Virginia

\section{By}

M. T. Berg, Department of Industrial Engineering

B. E. Reed, Department of Civil Engineering M. Gabr, Department of Civil Engineering West Virginia University

Mograntown, West Virginia 


\subsection{EXECUTIVE SUMMARY}

It is estimated that over 3700 hazardous waste sites are under the jurisdiction of the Department of Energy (DOE). Over the next 30 years, the Department of Energy (DOE) is committed to bringing all its facilities into compliance with applicable Federal, State, and local environmental laws and regulations. To perform this clean-up effort in the most efficient manner at each site will require that DOE managers have access to all available information on pertinent technologies; i.e., to aid in maximum technology transfer. The purpose of this effort is to systematically develop a database of those currently available and emerging clean-up technologies.

The development of a database of those currently available and emerging clean-up technologies is to be done in several phases: 1) A systems approach, 2) data collection, and 3) software development. Although the project officially started October 1, 1992, our award did not arrive until December, 1992. Thus, our main effort in the first quarter was 1) the recruitment of graduate research assistants, 2) the organization of project responsibilities, and 3) the procurement of software. In the second quarter we have 1) began an initial screening of DOE hazardous waste sites; 2) developed a conceptual model to classify DOE hazardous waste problems, and 3) developed an initial formulation of the structure (or fields) of the database. In the third quarter a user interface was developed to input and retrieve data from the database. This user interface was coded in FOXPRO 2.5 for DOS. Using the conceptual model and the structure of the database developed in quarter II, the user interface allows a user to input, search, retrieve and print records from the database via a menu driven system.

\subsection{BACKGROUND}

It is estimated that over 3700 hazardous waste sites are under the jurisdiction of the Department of Energy (DOE). These sites were primarily generated from 45 years worth of environmental pollution from the design and manufacture of nuclear materials and weapons, and contain numerous types of wastes including: 1) volatile, low-volatile and nonvolatile organics, 2) radionuclides (e.g., uranium, plutonium and cesium), 3) nonradioactive heavy metals (e.g., chromium, nickel, and lead), and 4) toxic chemicals. These contaminants affect several media including soils (saturated and unsaturated), groundwater, vegetation, and air. Numerous and diverse DOE hazardous waste sites can be enumerated from soils contaminated by organics such as trichloroethylene (TCE) and perchloroethylene (PCE) at the Savannah River site to biota and vegetation contaminated by radionuclides such as radiocesium and radiostrontium at the Oak Ridge site.

Over the next 30 years, the Department of Energy (DOE) is committed to bringing all its facilities into compliance with applicable Federal, State, and local environmental laws and regulations. This clean-up task is quite complex involving numerous sites cuntaining various radioactive, organic and inorganic contaminants. To perform this clean-up effort in the most efficient manner at each site will require that DOE managers have access to all available information on pertinent technologies; i.e., to aid in maximum technology transfer. The purpose of this effort is to systematically develop a database of those currently available and emerging clean-up technologies. 
The construction of a database of clean-up technologies requires a systematic development of those steps necessary to achieve clean-up objectives. These steps and associated technology groupings are given in Figure 1.

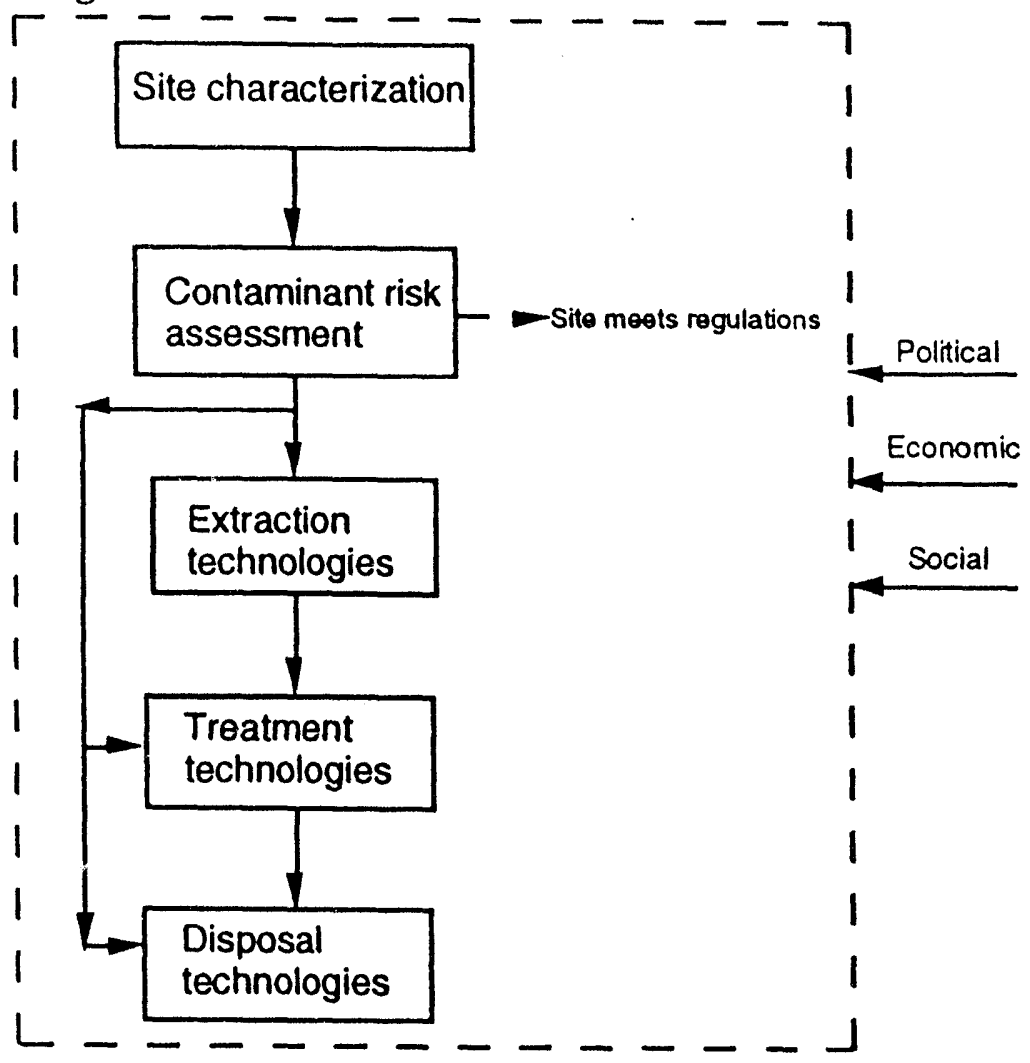

Figure 1: Division of technologies based on primary steps necessary for clean-up

The initial step in assessing a potential hazardous waste site is the characterization and identification of the type and extent of the contamination. In this characterization step technologies are required both for in-situ and ex-situ assessment of contamination levels. Once site characterization is performed, the risks posed by the contamination must be assessed. This step necessitates the use of mathematical models to predict contaminant fate and subsequent impact on local populations. Assuming that a risk exists, technologies must then be examined (or developed) to either extract the contaminant from the fostering media for treatment and/or disposal, treat the contaminant in-situ, or directly dispose of the contaminant. Furthermore, at each step in the clean-up process decisions regarding technology choices must be made within the existing political, economic, and social climate.

Using the conceptual approach given in Figure 1, a systematic assessment of available and emerging technologies in each area will be developed. This will be accomplished for each area by the development of a database of both current and emerging technologies. An example to illustrate the concept of such a database is given in Figure 2. In this simple example, the user of the database may be interested in investigating the available technologies for cleaning up a soil contaminated by dioxin (TCDD). In this case, information regarding soil extraction and treatment procedures is organized such that the user can extract pertinent clean-up information. 


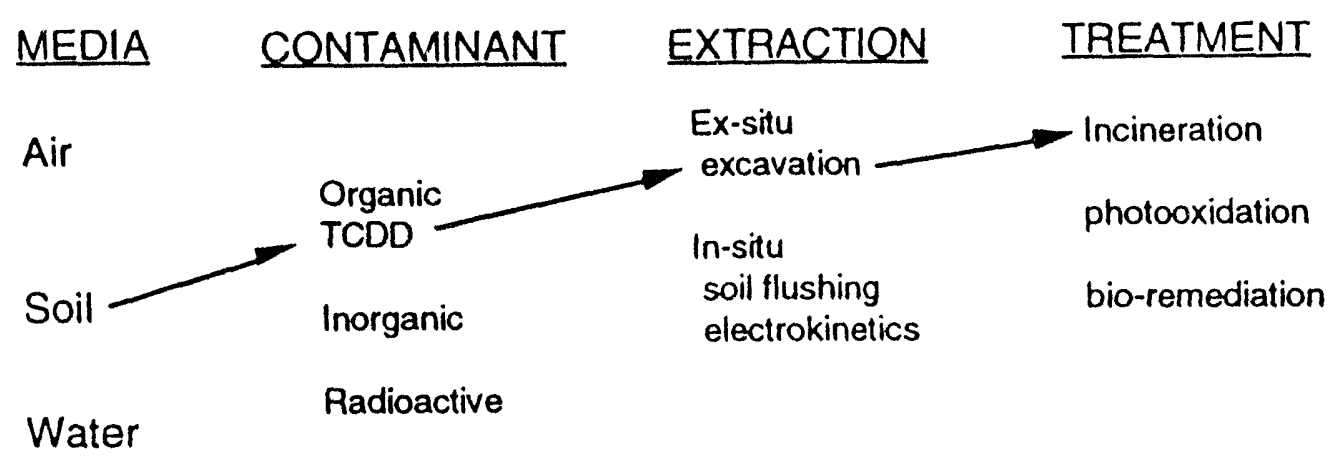

Figure 2: Conceptual view of database construction in which the arrows indicate one path explored for the clean-up of 2,3,7,8-Tetrachlorodibenzo-p-dioxin (TCDD)

It is envisioned in the initial phase of development that a DOE manager via a computer terminal will have access to information in the database via a query system. This system will allow the user to access the different available technologies for each step in the clean-up process, and extract pertinent information on how to proceed and obtain more detailed information. A natural result of such a systematic assessment is the discovery of where clean-up capabilities are lacking. Thus, as the process proceeds, a database will be compiled of those clean-up processes that are in need of further research.

\subsection{METHODOLOGY}

The development of a database of hazardous waste technologies will be carried out in several phases:

Phase I: A systems approach. This phase involves a systematic development of the components to be included in the database. The approach will drive the development of the computer database. The following sub-tasks are to be performed:

1. An initial screening of DOE hazardous waste sites;

2. The development of a conceptual model to classify DOE hazardous waste problems;

3. An initial formulation of the structure (or fields) of the database.

Phase II: Data collection. In this phase a complete review of the current clean-up technologies will be done. This review will be driven by the database structure developed in phase I (sub-task 3), and will be accomplished through a comprehensive literature review, discussions with clean-up experts, and possible hazardous waste site visitations. 
Phase III: Software development. In parallel with the data collection phase of this work, a computer based database is to be developed. This includes a data storage/retrieval system along with user friendly access software. Several main sub-tasks are to be performed including:

1. Development of a user friendly front end (i.e., user interface);

2. Development of the structure of the database;

3. Input of the technologies collected in the data collection phase;

4. Software verification and testing.

\subsection{RESULTS AND DISCUSSION}

Although the project officially started October 1, 1992, our award did not arrive until December, 1992. Thus, our main effort in the first quarter was 1) the recruitment of graduate research assistants, 2) the organization of project responsibilities, and 3) the procurement of software. As of January, 1992 we have recruited several graduate students who will function both to aid in the collection of data (phase II) and to work on software development. We have also organized project responsibilities toward the collection of data. All faculty will be responsible for the collection of data on characterization, extraction, treatment and disposal technologies in their field of expertise. Such data will be reported to the P.I. for integration into the database. We have also selected and ordered database software for the project. We have selected the relational database software FOX PRO 2.5 as our development package. This software will be run on an Intel 486 based computer.

In quarter II we have 1) began an initial screening of DOE hazardous waste sites, 2) developed a conceptual model to classify DOE hazardous waste problems, and 3) developed an initial formulation of the structure (or fields) of the database. An initial screening of DOE hazardous waste sites was initiated using the DOE Environmental Restoration and Waste Management Five Year Plan ${ }^{+}$. This report, however, gives only very general information on contaminated DOE sites. For example, information is given on the clean-up of volatile organics in saturated soils at the DOE's Savannah River site. The levels and extent of contamination are not given. We are in the process of searching for site specific data on the extent and level of contamination at specific sites.

In order to identify remediation technologies applicable to specific DOE sites, we have developed a conceptual model to classify DOE hazardous waste problems. This entails first the development of a general classification of hazardous waste problems. This general classification is simply a division of contamination problems based on 1) the media in which the pollutant resides, and 2) the chemical characteristics of the pollutants. This division is illustrated by the matrix given in Figure 3 in which the bold boxes indicate a class of technologies used to remediate sites with the given pollutant/media characteristics. Thus, for each step in the remediation process (i.e., characterization, extraction, and treatment) there exists sets of potential technologies that can address the specific problems. The choice of this division was based on

- USDOE, 1991. Environmental Restoration and Waste Management Five Year Plan. DOE/S-0090P. 
the behavior of pollutants in the environment. Pollutant behavior is driven by both its physical/chemical haracteristics and the media in which it resides (i.e., pollutant fate and transport are driven by media pollutant interactions along with pollutant characteristics). These fate and transport characteristics will drive the potential technologies that will be applicable for remediation. For example, pollutants which reside in soils and are tightly bound, hydrophobic, and not susceptible to bioremediation (e.g., dioxins) can be remediated using similar approaches. In this case, this class of pollutants is typically excavated and treated ex-situ via thermal or chemical technologies for organics or possible immobilization for inorganics.

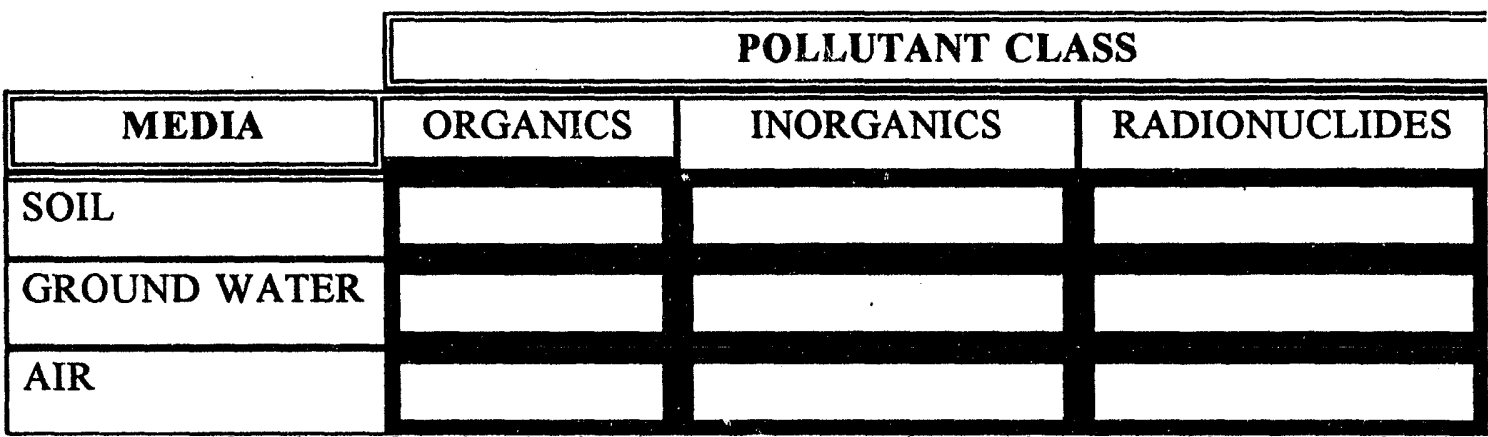

Figure 3: Classification of technologies into groups used to address combinations of different pollutants with different media.

Within each pollutant class we have further grouped chemicals with similar physical and/or chemical characteristics; i.e., groups of chemicals which tend to have similar behavior in the environment. These groups are given in Table $1^{+++}$. In a similar fashion the media in which the pollutant resides can be subdivided based on its affect on pollutant behavior. In Table 2 we list several factors affecting pollutant behavior in soil, groundwater, and air (Note: this is not intented to be a complete list of factors). Thus, by developing classifications for both the pollutant and the respective media it resides in, we are reducing the subset of remediation technologies that need be explored for a particular circumstance.

For specific hazardous waste problems (i.e., pollutants in specific media) we envision candidate alternative remediation strategies. These alternative remediation technologies themselves can be categorized based on the processes used in remediation. We have categorized remediation technologies into four general classes:

1) physical/chemical

2) thermal

3) biological, and

4) immobilization.

${ }_{+}^{+}$EPA, 1989. Superfund Treatability Clearinghouse Abstracts. EPA/540/2-89/001. 
Within each technology class there exists several specific remediation technologies which can be applied at each stage of the remediation process (i.e., characterization, extraction, treatment and disposal). Physical/chemical technologies include such processes as dehalogenation, air stripping, and chemical sorption; thermal technologies include such processes as vitrification, incineration and fluidized bed combustion; biological technologies include aerobic and anaerobic decomposition; immobilization includes such processes as cement solidification and carbonate immobilization.

Table 1

Grouping of Pollutants based on behavior in the environment.

\begin{tabular}{|l|c|}
\hline \multicolumn{1}{|c|}{ Pollutant Class } & Example \\
\hline \hline Halogenated non-polar aromatics & Chlorobenzene \\
\hline PCB's, halogenated dioxins and furans & $\begin{array}{c}\text { Tetrachlorodibenzo- } \\
\text { p-dicxin }\end{array}$ \\
\hline Halogenated phenols, cresols, amines, and other polar aromatics & Tetrachlorophenol \\
\hline Halogenated aliphatic compounds & Vinyl chloride \\
\hline Halogenated cyclic aliphatics, ethers, esters, and keytones & Toxaphene \\
\hline Nitrated aromatic and aliphatic compounds & Trinitrotoluene \\
\hline Heterocyclics and simple non-halogenated aromatics Benzene & Naphthalene \\
\hline Polynuclear aromatics & Methanol \\
\hline Other polar non-halogenated organic compounds & Iron \\
\hline Non-volatile metals & Mercury \\
\hline Volatile metals & Sulfate \\
\hline Other inorganics & Methyl Propane \\
\hline Other organics & Cs-137 \\
\hline Radionuclides & \\
\hline
\end{tabular}


Table 2

Division of media with several factors affecting pollutant behavior in the environment.

\begin{tabular}{|c|l|}
\hline Media & \multicolumn{1}{|c|}{ Factor Affecting Transport } \\
\hline \hline SOIL & Texture (\%sand,\%silt,\%clay) \\
\hline & Grain size distribution \\
\hline & $\mathrm{pH}$ \\
\hline & Hydraulic conductivity \\
\hline & Organic content \\
\hline & Moisture content \\
\hline & Temperature \\
\hline & Biota \\
\hline $\begin{array}{c}\text { Cation exchange } \\
\text { capacity }\end{array}$ & Bulk density \\
\hline GROUND WATER & Dissolved organic content \\
\hline & Colloidal particals \\
\hline & Dissolved oxygen content \\
\hline & Temperature \\
\hline & Biota \\
\hline & pH \\
\hline & Pressure \\
\hline & Temperature \\
\hline AIR & Humidity \\
\hline & \\
\hline
\end{tabular}

Based on the classification schemes for pollutants, media, and remediation technologies described above, we have developed an initial structure for the database. This structure is illustrated in Figure 4. To date we have created the structure for five relational databases. These databases will contain specific information regarding pollutants and the alternative technologies available for remediation. In conjunction to these databases, a User interface, search routines and a report generator will be developed. The User interface will be used both in the data gathering phase in which the data is input in each database (i.e., the development of input screens) along with User interface during execution of search routines. The search routines will entail programs that generate a "best" match of site specific data on the pollutant and the media it resides in to potential remediation technologies. Finally, report generation routines will be developed to extract information from the database. We are currently in the data gathering phase of the project with only minimal development of screens for User input of information. 


\begin{tabular}{|c|c|}
\hline $\begin{array}{l}\text { RELATIONAL DATABASES } \\
\text {-Pollutant classification } \\
\text {-Data on inorganic/radionuclides } \\
\text {-Data on organics } \\
\text {-Technology classification } \\
\text {-Technolov demonstration }\end{array}$ & $\begin{array}{l}\text { SOFTWARE DEVELOPMENT } \\
\text {-User interface } \\
\text {-Search routines } \\
\text {-Report generation }\end{array}$ \\
\hline
\end{tabular}

Figure 4: Components of database

Tables 3-7 show the initial structure (or fields) of the relational databases given in Figure 4. Tables 3-5 represent the structure used to collect data on specific inorganic, organic and radioactive pollutants. First, a classification of all pollutants is accomplished by the database represented by Table 3. The groups (i.e., field 2) are assigned based on the classification scheme developed previous (see Table 1). Tables 4-5 then tabulate specific data regarding each pollutant. This data gives information regarding the physical and chemical characteristics of each pollutant that are important for assessing pollutant behavior in different media. Such data includes a pollutants volatility, solubility, molecular weight, etc. Tables 6-7 give the organization for database construction of remediation technologies. Two databases of remediation technologies are to be constructed: 1) a general classification of technologies used to address contaminated sies (Table 6), and 2) a database of technologies already demonstrated at the laboratory, bench or field scale.

Table 3

Division of Pollutants into Classes

\begin{tabular}{|c|l|l|}
\hline Field & Field Name & Description \\
\hline \hline 1 & NUM & Pollutant class number (e.g., P01,P02, etc.) \\
\hline 2 & GROUP & Name of Pollutant Class identified by NUM \\
\hline 3 & CLASS & General class: organic/inorganic/radionuclide \\
\hline 4 & POLLUTANT & Specific pollutant \\
\hline
\end{tabular}


Table 4

Physical and chemical data for specific inorganic and radioactive pollutants

\begin{tabular}{|c|l|l|}
\hline Field & \multicolumn{1}{|c|}{ Field Name } & Description \\
\hline \hline 1 & POLLUTANT & Specific pollutant \\
\hline 2 & NUM & Pollutant class number (e.g., P01,P02, etc.) \\
\hline 3 & REDOX_NUM & Oxidation number \\
\hline 4 & BOIL_PT & Boiling point $\left(\mathrm{C}^{0}\right)$ \\
\hline 5 & MELT_PT & Melting point $\left(\mathrm{C}^{0}\right)$ \\
\hline 6 & DENSITY & Density of pollutant $(\mathrm{g} / \mathrm{ml})$ \\
\hline 7 & SOLUBILITY & Solubility of pollutant $(\mathrm{g} / 100 \mathrm{ml})$ \\
\hline 8 & DIFFUSION & Diffusion coefficient $\left(\mathrm{Cm}^{2} / \mathrm{sec}\right)$ \\
\hline 9 & GIBBS & Standard state free energy $(\mathrm{kcal} / \mathrm{g}$-mole) \\
\hline 10 & VAPOR_PRESS & Vapor pressure (atm) \\
\hline 11 & HENRY_CON & Henry's law constant $\left(\mathrm{atm}-\mathrm{m}^{3} / \mathrm{mole}\right)$ \\
\hline 12 & HALF_LIFE & Half life for radionuclide (year) \\
\hline 13 & DAUGHTER & Daughter produced by decay of radionuclide \\
\hline 14 & BIO_EFFECT & Description of biological effect of pollutant \\
\hline
\end{tabular}


Table 5

Physical and chemical data for specific organic pollutants

\begin{tabular}{|c|c|c|}
\hline Field & Field Name & Description \\
\hline 1 & POLLUTANT & Specific pollutant \\
\hline 2 & NUM & Pollutant class number (e.g., P01,P02, etc.) \\
\hline 3 & MOL_WT & Molecular weight (g/mole) \\
\hline 4 & BOIL_PT & Boiling point $\left(\mathrm{C}^{0}\right)$ \\
\hline 5 & MELT_PT & Melting point $\left(\mathrm{C}^{0}\right)$ \\
\hline 6 & DENSITY & Density of pollutant $(\mathrm{g} / \mathrm{ml})$ \\
\hline 7 & SOLUBILITY & Solubility of pollutant $(\mathrm{g} / 100 \mathrm{ml})$ \\
\hline 8 & DIFFUSION & Diffusion coefficient $\left(\mathrm{Cm}^{2} / \mathrm{sec}\right)$ \\
\hline 9 & GIBBS & Standard state free energy (kcal/g-mole) \\
\hline 10 & VAPOR_PRESS & Vapor pressure (atm) \\
\hline 11 & HENRY_CON & Henry's law constant (atm- $\mathrm{m}^{3} / \mathrm{mole}$ ) \\
\hline 12 & LOG_OCT_H2 & Log of octanol to water partition coefficient \\
\hline 13 & PHOTODEG & Ability of organic to photo degrade (Yes/No) \\
\hline 14 & BIODEGRAD & Ability of organic to biodegrade (Yes/No) \\
\hline 15 & BIO_AGENTS & Specific biological agents found to degrade \\
\hline 16 & POLAR & Is organic polar (Yes/No) \\
\hline 17 & BIO_EFFECT & Description of biological effect of pollutant \\
\hline
\end{tabular}

Table 6

Classification of Technologies used to remediate contaminated sites

\begin{tabular}{|c|l|l|}
\hline Field & \multicolumn{1}{|c|}{ Field Name } & Description \\
\hline \hline 1 & REMED_PHAS & $\begin{array}{l}\text { Phase of remediation: } \\
\text { Characterization/Extraction/Treatment/Disposal }\end{array}$ \\
\hline 2 & TECH_GROUP & $\begin{array}{l}\text { Technology group: Physical- } \\
\text { Chemical/Biological/Thermal/Immobilization }\end{array}$ \\
\hline 3 & PROCESS & Specific process used under TECH_GROUP \\
\hline 4 & MEDIA & Process Media: Soil/Ground water/ Air \\
\hline 5 & CONTAM_GRP & Pollutant class number (e.g., P01,P02, etc.) \\
\hline 6 & REF & References in which technology is described \\
\hline
\end{tabular}


Table 7

Compilation of technologies that have been demonstarted at laboratory, bench or field scale

\begin{tabular}{|c|l|l|}
\hline Field & Field Name & \multicolumn{1}{|c|}{ Description } \\
\hline \hline 1 & TECHNOLOGY & Title of technology \\
\hline 2 & DEVELOPER & Developer of technoiogy \\
\hline 3 & MEDIA & Process Media: Soil/Ground water/ Air \\
\hline 4 & DOMAIN & Remediation domain: In-situ/Ex-situ \\
\hline 5 & SCALE & Scale of application: Lab/Bench/Field \\
\hline 6 & DESCRIPTION & Description of technology \\
\hline 7 & APP_WASTE & General class of applicable waste \\
\hline 8 & SPEC_WASTE & Specific waste in which technology is applied \\
\hline 9 & WAST_CLASS & Pollutant class number (e.g., P01,P02, etc.) \\
\hline 10 & TECH_GROUP & Technology group: Physical- \\
& & Chemical/Biological/Thermal/Immobilization \\
\hline 11 & PROCESS & Specific process used under TECH_GROUP \\
\hline 12 & SITE & Site description (if any) \\
\hline 13 & STATUS & Status of work \\
\hline 14 & CONTACTS & Specific persons to contact \\
\hline 15 & DOCUMENTS & Publications of work \\
\hline 16 & COST & Cost data \\
\hline 17 & REF & Reference in which information was obtained \\
\hline
\end{tabular}

In the third quarter, we used the conceptual model and database structure to develop a user interface. The user interface is a set of routines coded in FOXPRO 2.5 that allows a user to input, retrieve, search and print any record in the database via a menu driven system. The development of a user interface is essential for efficient use of the database along with the protection of data. The code developed to interface between the user and the database is given in the attached Appendix A. In conjunction with software development, we have continued gathering data to input into the database. We are currently gathering and inputing data on general descriptions of each technology (as given in Table 6). Furthermore, we are in the process of assessing the available information via external databases (e.g., ATTIC, PROTEC, etc.). We hope to reduce data collection tasks by downloading available information from existing databases. 
In the fourth quarter, we have continued both the development of software, and the data collection phase. The first version of user interface software in FOXPRO 2.5 has been completed. The initial introductory screen is given in Figure 5. This screen welcomes the user and designates the five main menu options as ADD, EDIT, SEARCH, EXIT and HELP. If the user chooses the ADD option from the main menu, a list of databases appears. The five accessible databases include organic pollutants (ORGANIC), inorganic pollutants (INORGANIC), radionuclide pollutants (RADIONUCLIDE), remediation technologies (TECHNOLOGY), and developed/demonstrated technologies (DEVELOP). The first three contain relevant chemical data on organic and inorganic pollutants. The TECHNOLOGY database gives general information on remediation technologies, and the DEVELOP database provides demonstrations of the technologies and site-specific information. Once the user selects a database, the program immediately provides a blank record in which to enter data.

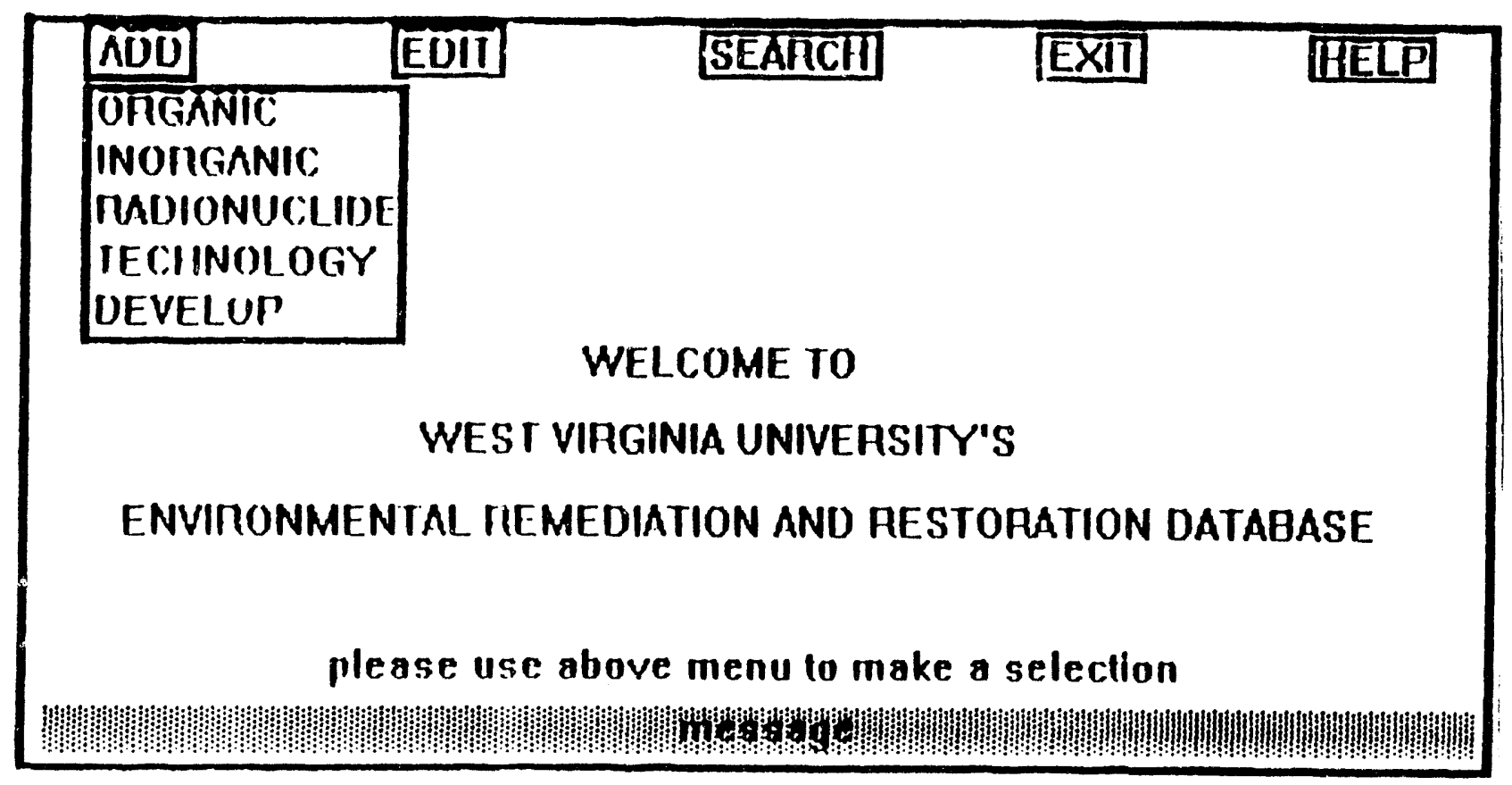

Figure 5. The Introductory Screen to the User Interface Software

The EDiT option from the main menu povides the same database choices. However, once a selection is made, a second screen appears which requests search criteria. The push button MAINMENU at the bottom of this screen will return to the main menu. If the SEARCH button is activated, the program will then use the specified criteria to locate the record and display it on a third screen. 
The display screen allows the user to browse the record but not make any changes. It then provides four alternatives: EDIT, DELETE, CANCEL, and PRINT RECORD. CANCEL will return to the search criteria input screen. DELETE will request double confirmation and then erase the displayed record. PRINT RECORD will allow the user to print the record to the screen or to the printer. The program controlling this print option calls a specific report (an .RPT file exists for each of the five databases). The program then transfers the field information from the appropriate database into the report format and sends the report to the requested destination. Finally, EDIT will bring the displayed record up on data entry screens where it can be edited.

On every data entry screen (purple screens) in both the ADD and EDIT mode, there are three common features. First, there are regions for entering the information. We found that certain programming features are best suited for certain data types. For example, when only one choice is to be selected from a few options, push buttons are convenient. However, check boxes are more suitable if multiple selections are possible. We also used checkboxes to activate AT...EDIT regions in which to edit memo fields. The second feature available is the HELP menu in the upper right hand corner of the screens. When this menu is activated, a program is called which will define any field for the user. It also provides the width and type of the field. At the conclusion of the inquiry, the program returns to the previous screen. Finally, at the bottom of each data entry screen, there are SAVE and CANCEL push buttons. If the user chooses SAVE, the changes to the record will be saved in the appropriate database. If CANCEL is chosen, the changes wil! be disregarded. The CANCEL option is also helpful if the user inadveriuntly selects the wrong database.

The programs for ADD and EDIT were designed to create a user-friendly interface which would allow input into the appropriate database without exposing the database format or fields. This approach lends itself to good quality control of the entered data. Programming was also done in such a way that any user error can be easily corrected, and there is always a CANCEL option. Furthermore, a great deal of consideration was taken into the color, type, and organization of the screens. While there is still some programming to be done, we feel that the database is currently at a level in which data entry will be convenient and efficient.

Extensive programming has been done to complete the ADD and EDIT options allowing the user to input, retrieve and edit data. Currently, the SEARCH option is disabled. The first version of the user interface code was tested by several users (i.e., individuals not participating in the development of the code). Feedback from these tests was used to improve the user interface.

The data collection phase continued with the collection of data for the technology database (i.e., Table 6). We have completed general and technical descriptions of biological and thermal remediation technologies. The general description provides the reader with easily understood information about a technology's process and concepts. This section is written at a level so that a person who is not an environmental engineer can get a basic idea of where and how the environmental technology can be applied. The technical description gives more detailed information on the process and theory behind the technology. The theory includes any important chemical equations or formulas that indicate how the technology works. This section also discusses factors that influence technology performance and limitations. Examples of these 
descriptions are found in Appendix B. We have also begun collecting information on immobilization and chemical remediation technologies.

\subsection{CONCLUSION}

The resources necessary for the development of a computer database of hazardous waste technologies have been assembled. In the second quarter, we have systematically developed the structure of the database. This structure was driven by the necessity to relate the physical and chemical data of specific hazardous waste sites to specific remediation technologies. We have created the databases (i.e., developed the fields) using Microsoft FOXPRO. We are in the data collection phase of the project. To date, we have entered into the database 296 specific pollutants, and 58 classes of technologies. At this time we are in the process of obtaining specific data on the pollutants entered (i.e., filling out Tables 5 and 6), and obtaining more information regarding specific technology classes (i.e., filling out Table 7). We have also begun entering data on technology demonstrations on the laboratory, bench and field scale. In the third quarter, we expended a great deal of effort on the development of a user interface. The user interface will allow the access and storage of information via a menu driven system. Once tested this system shall allow any user (not necessarily familiar with FOXPRO) to interface with the database including inputing, searching and printing database records. In the fourth quarter, we continued the development of user interface software including refinements to the code based on feedback from external reviewers. We have completed an initial collection of data for the technology database on both biological and thermal remediation and treatment. In this quarter, we have continued software and data collection efforts. It is critical that in the next quarter we re-assess the objectives of this project and how this objective fits in with the goals at DOE. 
APPENDIX A

User Interface Code in FOXPRO 2.5 
MAIN menu program: Runs main menu and passes control to

selected function.

MEMORY VARIABLES :

done: logical var done $=. T$. stops executing of program

PUBLIC done

done $=. F$.

* Run main menu definition

DO c: \metc\database \prog\maindef.prg

DO WHILE (.NOT. done)

SET TALK OFF

* Turn off FOXPRO menu

SET SYSMENU OFF

* Display logo

ACTIVATE WINDOW name

(1) 13,34 SAY "WELCOME TO" COLOR GR+/B

@ 15,26 SAY "WEST VIRGINIA UNIVERSITY'S" COLOR GR+/B

@ 17,14 SAY "ENVIRONMENTAL REMEDIATION AND RESTORATION DATABASE" COLOR GR $+/ B$

@ 9,14 SAY "(Please use the above menu to make a selection.)"

* Run the main menu until done is set to true.

ACTIVATE MENU main

ENDDO

*

* Function to control execution of main menu

PROCEDURE control

PARAMETER choice, bar

*Find the choice made: EDIT/DEL, ADD, SEARCH, QUIT

DO CASE

* Stop execution of the program

CASE choice = 'quit'

done $=. \mathrm{T}$.

* reset FOXPRO environment

CLEAR READ ALL

CLEAR WINDOW ALL

SET ESCAPE ON

SET TALK ON

CLOSE DATABASES

* Add a record to the database

CASE choice $=$ 'add'

DO editrec WITH bar, .T.;

IN $c: \backslash$ metc $\backslash$ database $\backslash$ prog $\backslash$ editrec.prg

* Edit existing data in the database

CASE choice = 'edit'

* Initialize memory variables used in editmenu

srchstring = SPACE (30)

DEACTIVATE WINDOW name

* Pass control to editmenu

DO editmenu WITH bar, srchstring;

IN $c: \backslash$ metcldatabase \prog $\backslash$ editmenu.prg

ACTIVFTE WINDOW name

(13,34 SAY "WELCOME TO" COLOR GR+/B

@ 15,26 SAY "WEST VIRGINIA UNIVERSITY'S" COLOR GR+/B

(a) 17,14 SAY "ENVIRONMENTAL REMEDIATION AND RESTORATION DATABASE";

STYLE 'BI' COLOR GR+/B

(9 9,14 SAY "(Please use the above menu to make a selection.)"

CASE choice = 'search'

WAIT WINDOW 'MENU CHOICE CURRENTLY DISABLED'

CASE choice = 'help'

WAIT WINDOW 'MENU CHOICE CURRENTLY DISABLED'

ENDCASE 


\section{RETURN}


* Define main menu for hazardous waste technology database

* Define a window and display initial greeting CLEAR

DEFINE WINDOW name FROM 0,0 TO 25,79 PANEL SHADOW;

COLOR SCHEME 1

* Define the menu used to select action

DEFINE MENU main IN WINDOW name

$\star$

DEFINE PAD addpad OF main PROMPT 'ADD' AT 0,3 COLOR SCHEME 6

DEFINE PAD editpad OF main PROMPT 'EDIT' AT 0,18 COLOR SCHEME 6

DEFINE PAD srchpad OF main PROMPT 'SEARCH' AT 0,33 COLOR SCHEME 6

DEFINE PAD endit OF main PROMPT 'EXIT' AT 0,48 COLOR SCHEME 6

DEFINE PAD helppad OF main PROMPT 'HELP' AT 0,63 COLOR SCHEME 6

*

ON PAD addpad OF main ACTIVATE POPUP add

ON PAD editpad OF main ACTIVATE POPUP edit

ON PAD srchpad OF main ACTIVATE POPUP search

ON PAD endit OF main ACTIVATE POPUP exit

ON PAD helppad OF main ACTIVATE POPUP help

*

* Define ADD popup/menu

$\star$

DEFINE POPUF add FROM 1,3 TN WINDOW name COLOR SCHEME 6

DEFINE BAR 1 OF add PROMPT 'ORGANIC';

MESSAGE 'Add an organic pollutant to database' COLOR , , , GR+/BR+

DEFINE BAR 2 OF add PROMPT 'INORGANIC';

MESSAGE 'Add an inorganic pollutant to' database' COLOR, , , GR+/BR+

DEFINE BAR 3 OF add PROMPT 'RADIONUCLIDE' ;

MESSAGE 'Add a radionuclide pollutant to database' COLOR, , , GR+/BR+

DEFINE BAR 4 OF add PROMPT 'TECHNOLOGY';

MESSAGE 'Add a general technology to database' COLOR , , , GR+/BR+

DEFINE BAR 5 OF add PROMPT 'DEVELOP';

MESSAGE 'Add a developed/demonstrated technology to database';

COLOR , , , GR+/BR+

ON SELECTION POPUP add DO control WITH 'add', BAR()

$\star$

* Define EDIT/DELETE popup/menu

DEFINE POPUP edit FROM 1,18 IN WINDOW name COLOR SCHEME 6

DEFINE BAR 1 OF edit PROMPT 'ORGANIC';

MESSAGE 'Delete/edit organic pollutant in database' COLOR, , , GR+/BR+

DEFINE BAR 2 OF edit PROMPT 'INORGANIC';

MESSAGE 'Delete/edit an inorganic pollutant to database' COLOR, , , GR+/BR+

DEFINE BAR 3 OF edit PROMPT 'RADIONUCLIDE';

MESSAGE 'Delete/edit a radionuclide pollutant to database' COLOR, , , GR+/BR+

DEFINE BAR 4 OF edit PROMPT 'TECHNOLOGY';

MESSAGE 'Delete/edit a general technology in database' COLOR, , , GR+/BR+

DEFINE BAR 5 OF edit PROMPT 'DEVELOP';

MESSAGE 'Delete/edit a developed/demonstrated technology in database';

COLOR , , , GR+/BR+

ON SELECTION POPUP edit DO control WITH 'edit', BAR()

*

$\star$

* Define search popup/menu

DEFINE POPUP search FROM 1,33 IN WINDOW name COLOR SCHEME 6

DEFINE BAR 1 OF searCh PROMPT 'SEARCH';

MESSAGE 'Search routines to be developed....' COLOR , , , GR+/BR+

$\star$

ON SELECTION POPUP search DO control WITH 'search', BAR() 
Define EXIT selection

DEFINE POPUP exit FROM 1,48 IN WINDOW name COLOR SCHEME 6 DEFINE BAR 1 OF exit PROMPT 'EXIT';

MESSAGE 'End database session' COLOR , , , GR+/BR+

JN SELECTION POPUP exit DO control WITH 'quit', 0

* Define HELP popup/menu

*

DEFINE POPUP helP FROM 1,63 IN WINDOW name COLOR SCHEME 6 DEFINE BAR 1 OF helP PROMPT 'HELP';

MESSAGE 'HELP routines to be developed....' COLOR , , ,GR+/BR+

JN SELECTION POPUP help DO control WITH 'help', BAR()

$\star$

RETURN 


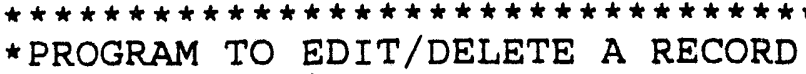 \\ PROCEDURE editmenU \\ PARAMETERS mchoice, srchstring}

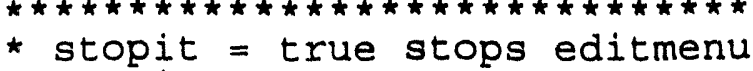

stopit $=$. . .

* Mem var for search/mainmenu

runchoice $=1$

DEFINE WINDOW editsearch FROM 0,0 TO 24,79 COLOR SCHEME 1

* Define POPUP for field choices for organic/inorganic database

DEFINE POPUP efield1 COLOR SCHEME 5

DEFINE BAR 1 OF efield1 PROMPT 'Pollutant

DEFINE BAR 2 OF efieldl PROMPT 'Chem. Abstract \#'

$\star$

* Define POPuP for field choices technology database

DEFINE POPUP efield2 COLOR SCHEME 5

DEFINE BAR 1 OF efield2 PROMPT 'Technology'

DEFINE BAR 2 OF efield2 PROMPT 'Subclass

$\star$

* Define popup for field choices for develop database

DEFINE POPUP efield3 COLOR SCHEME 5

DEFINE BAR 1 OF efield3 PROMPT 'Tech. Name'

DEFINE BAR 2 OF efield3 PROMPT 'Technology'

DEFINE BAR 3 OF efield3 PROMPT 'Developer'

DEFINE BAR 4 OF efield3 PROMPT 'Site

* Execute edit menu until user chooses to go back to main menu.

DO WHILE (.NOT. StOpit)

* Test in is a boolean variabie that determines if user

* has indexed on a field

test in $=. F$.

* FChoice is the field that the user chooses

fchoice=space (15)

* hold $=$ true means searchstring has been chosen

hold $=. F$.

* Set up a screen to get info from user

ACTIVATE WINDOW editsearch

Q 3,22 SAY "Input search character string:"

(a 7,8 SAY "ChOose search criterion and press ENTER or double-click mouse:"

(a) 16,29 SAY "Selections made:"

DO CASE

* Set up search screen for organic database.

CASE mchoice $=1$

(a) 1,14 SAY "SEARCH FOR RECORD IN ORGANIC POLLUTANT DATABASE" COLOR GR+/B

(a) 17,10 TO 20,65

(18, 11 SAY "SEARCH STRING:"

(19), 11 SAY "SEARCH CRITERION:"

* If value is to be held, reprint value in box.

IF hold $=$.T.

(1) 4,22 SAY srchstring

(a) 18,30 SAY srchstring

* Otherwise, get the new value and print in box.

ELSE

@ 4,22 GET srchstring VALID dispitem(18,30, srchstring)

ENDIF

(a) 8,25 GET fchoice PICTURE "@\&N" POPUP efieldl;

SIZE 6,25 VALID tindex (@test_in)

(a) 22,29 GET runchoice FUNCTION ${ }^{-1} * \mathrm{H}$ SEARCH; MAINMENU ';

COLOR , , , GR+/R

READ CYCLE 
* Set up search screen for inorganic/radionuclide database.

CASE (mchoice $=2$. or . mchoice $=3$ )

@ 1,13 SAY "SEARCH FOR RECORD IN INORGANIC/RADIONUCLIDE DATABASE" COLOR $\mathrm{GR}+/ \mathrm{B}$

(a) 17,10 TO 20,65

(18) 18, 11 SAY "SEARCH STRING:"

(4) 19,11 SAY "SEARCH CRITERION:"

* If value is to be held, reprint value in box.

IF hold $=$. T.

(1) 4,22 SAY srchstring

(a) 18,30 SAY srchstring

* Otherwise, get the new value and print in box.

ELSE

(1) 4,22 GET srchstring VALID dispitem(18,30, srchstring)

ENDIF

@ 8,25 GET Echoice PICTURE "@\&N" POPUP efieldl;

SIZE 6,25 VALID tindex (@test in)

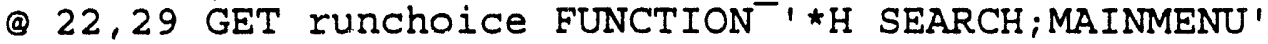

READ CYCLE

* Set up search screen for technology database.

CASE mchoice $=4$

Q 1,17 SAY "SEARCH FOR RECORD IN TECHNOLOGY DATABASE" COLOR GR+/B

* Define Popup for field choices

(a) 17,10 TO 20,65

(1) 18,11 SAY "SEARCH STRING:"

@ 19, II SAY "SEARCH CRITERION:"

* If value is to be held, reprint value in box.

If hold $=$.T.

(@) 4,22 SAY srchstring

Q 18,30 SAY srchstring

* Otherwise, get the new value and print in box.

ELSE

(1) 4,22 GET srchstring VALID dispitem(18,30, srchstring) ENDIF

@ 8,25 GET fchoice PICTURE "@\&N" POPUP efield2;

SIZE 6,25 VALID tindex(@test_in)

(1) 22,29 GET runchoice FUNCTION ${ }^{-1} * \mathrm{H}$ SEARCH; MAINMENU'

READ CYCLE

* Set up search screen for develop database.

CASE mchoice $=5$

@ 1,19 SAY "SEARCH FOR RECORD IN DEVELOP DATABASE" COLOR GR+/B

* Read runchoice=goto mainmenu or run search

(Q) 17,10 TO 20,65

(18) 18,11 SAY "SEARCH STRING:"

(1) 19,11 SAY "SEARCH CRITERION:"

* If value is to be held, reprint value in box.

IF hold $=$. T.

(1) 4,22 SAY srchstring

(a) 18,30 SAY srchstring

* Otherwise, get the new value and print in box.

ELSE

@ 4,22 GET srchstring VALID dispitem(18,30, srchstring)

ENDIF

(9) 8,25 GET fchoice PICTURE "@\&N" POPUP efield3;

SIZE 6,25 VALID tindex(@test in)

(a) 22,29 GET runchoice FUNCTION ${ }^{-1} \star^{H}$ SEARCH; MAINMENU'

ENDCASE

READ CYCLE

* Convert field choices in POPUP to match field choices in database files. DO CASE

CASE fchoice $=$ 'Chem. Abstract \#' fchoice = 'CAS NUM'

CASE fchoice $=$ 'Tech. Name' 
* If the user chooses the search button, control is passed

* to the search routine.

IF (runchoice = 1)

* If there is a search criterion selected do the search

IF (test in = .T.)

DEACTIVATE WINDOW editsearch

DO search WITH mchoice, fchoice, srchstring

* Otherwise display a message and go back to editmenu.

ELSE

* If the user forgets to enter a field to search on, a screen

* appears to instruct the user to do so.

WAIT WINDOW 'YOU MUST CHOOSE A SEARCH CRITERION. PresS ENTER tO continue.'

* Hold is first initialized as .F.

* However, if the user does not enter a field on the first screen in

* editmenu, hold becomes.T. so that the value of the searchstring

* is not lost.

hold $=. T$.

ENDIF

* Otherwise, return to the mainmenu

ELSE

DEACTIVATE WINDOW editsearch

* stopit $=. T$.

EXIT

ENDIF

ENDDO

RETURN

Function testindex determines if a user has indexed on a field

Prints fchoice in the "Selections Made" box and returns true

if a user has indexed on a field

FUNCTION tindex

PARAMETER test in

test in $=$.T.

@ 19,30 SAY f́choice

RETURN test in

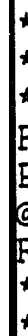

* Function dispitem prints the value of the srchstring in the

"Selections Made" box

FUNCTION dispitem

PARAMETER $x, y$, item

Q $x, y$ SAY item

RETURN

$\star$

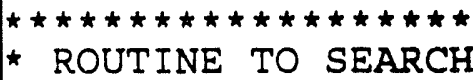

PROCEDURE SEarCh

PARAMETERS mchoice, fchoice, srchstring

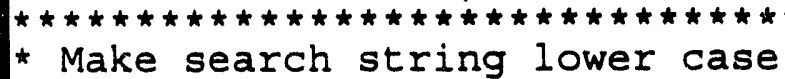

srchstring = ALLTRIM (LOWER (srchstring))

DEFINE WINDOW dispsrch FROM 0,0 TO 24,79 COLOR SCHEME 5

CLEAR

ACTIVATE WINDOW dispsrch

* Find nearest record

SET NEAR ON

* Open the specified database

DO CASE

CASE mchoice $=1$

USE $C: \backslash M E T C \backslash D A T A B A S E \backslash D A T A \backslash O R G A N \_C H . D B F$ 
CASE (mchoice $=2$.OR. mchoice $=3$ )

USE C: \METC\DATABASE\DATA IINOR_RAD.DBF

CASE mchoice $=4$

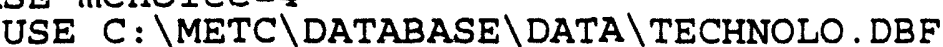

CASE mChoice $=5$

USE $C: \backslash M E T C \backslash D A T A B A S E \backslash D A T A \backslash D E V E L O P . D B F$

ENDCASE

* Choice index to order database

SET ORDER TO TAG \&fchoice

SEEK srchstring

* If the string is found, then display the record

IF (FOUND() .OR. (.NOT. EOF()))

DEACTIVATE WINDOW dispsrch

DO disprec WITH mchoice

* If the string is not found, message will appear, and control

* is passed to editmenu

ELSE

* If the search string is not found a screen appears and informs the user.

WAIT WINDOW 'search string not found! Press ENTER to continue.' DEACTIVATE WINDOW dispsrch

* Reassign search string as a character value before passing

* control back to editmenu.

srchstring = SPACE $(30)$

ENDIF

RETURN

SET EXACT ON 
* PROCEDURE TO EDIT RECORD

PROCEDURE editreC

PARAMETERS mchoice, addopt, recnum

$\star \star \star \star \star \star \star \star \star \star \star \star \star \star \star ~$
appblank $=$. F

continue $=. T$.

whichelp $=0$

whatmemo $=0$

okcancel $=0$

sacancel $=0$

screen $=1$

change $1=0$

change $2=0$

pushd $=0$

pushtg $=0$

pushsc $=0$

bioopt $=0$

check $=0$

picks $=0$

pickw $=0$

picka $=0$

pickc $=0$

picke $=0$

pickt $=0$

pickd $=0$

picalph $=0$

picbeta $=0$

picgamm $=0$

checkba $=0$

checkbe $=0$

checkr $=0$

ymedia = SPACE $(6)$

Ypollut $=$ SPACE $(7)$

DEFINE WINDOW record FROM 0,0 TO 24,79 COLOR SCHEME 5 ACTIVATE WINDOW record

* Define help popup

DEFINE MENU fhelp IN WINDOW record

DEFINE PAD helppad OF thelp PROMPT 'HELP' AT 0,70

ON PAD helppad OF thelp ACTIVATE POPUP helpl

DEFINE POPUP helP1 FROM 1,67 IN WINDOW record COLOR SCHEME 1

DEFINE BAR 1 OF helpl PROMPT 'Field Definitions' COLOR SCHEME 10

DEFINE BAR 2 OF help1 PROMPT 'Cancel' COLOR SCHEME 10

ON SELECTION POPUP helpl DO helpit WITH BAR(), 1

* Open database which user wants if add was chosen in mainmenu

* and scatter the data to empty memory variables.

IF addopt $=$. T.

DO CASE

CASE mchoice $=1$

USE C: \METC\DATABASE\DATA \ORGAN CH.DBF

CASE (mchoice $=2$. OR. mchoice $=\overline{3}$ )

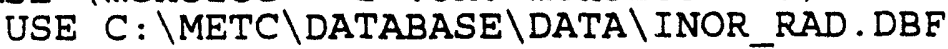

CASE mchoice $=4$

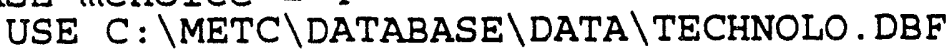

CASE mChOice $=5$

ENDCASE

USE C: \METC\DATABASE\DATA \DEVELOP.DBF

GOTO BOTTOM

SCATTER MEMO MEMVAR BLANK

* Otherwise, scatter the data to the existing memory variables ELSE

SCATTER MEMO MEMVAR 


\section{ENDIF}

* This segment will print the current contents of the field * on the designated screen.

DO CASE

* What to do with checkboxes in radionuclide database.

CASE mchoice $=3$

* Write the current contents of the specified field on the

* screen beside the checkboxes.

IF m.emission_1 = 'alpha'

$\mathrm{m}$. picalph $=-1$

(Q) 18,32 SAY m.emission_1

ENDIF

IF m.emission_2 = 'beta'

m. picbeta $=-1$

(19,32 SAY m.emission_2

ENDIF

IF m.emission 3 = 'gamma'

m. picgamm $=-1$

(a) 20,32 SAY m.emission_3

ENDIF

* What to do with push buttons and checkboxes in

* technology database.

CASE mchoice $=4$

* Write the current contents of the specified field on the

* screen above the push buttons.

(1) 7,12 SAY m.domain

(a) 5,30 SAY m.tech group

* Write the current contents of the specified field on the

* screen beside the checkboxes.

IF m.medial = 'soil'

m.picks $=1$

(1) 10,34 SAY m.medial

ENDIF

IF m.media2 = 'water'

$\mathrm{m} \cdot \mathrm{pickw}=1$

(a) 11,34 SAY m.media2

ENDIF

IF m. media $3=$ 'air'

m. picka $=1$

@ 12,34 SAY m.media3

ENDIF

IF m.rem phase l = 'characterization'

$\mathrm{m} \cdot \mathrm{pick} \overline{\mathrm{c}}=1$

(a) 14,50 SAY m.rem phase1

ENDIF

IF m.rem_phase 2 = 'extraction'

m.picke $=1$

(a) 15,50 SAY m.rem phase2

ENDIF

IF m.rem phase 3 = 'treatment '

m.pick $\bar{E}^{=}=1$

(@) 16,50 SAY m.rem_phase3

ENDIF

IF $m$.rem phase4 = 'disposal $'$

m. pick $\bar{d}=1$ ENDIF

(a) 17,50 SAY m.rem_phase4

\section{SNDCASE}

CTIVATE MENU thelp NOWAIT

Display record to edit or add

DO CASE

* Setup screen to edit/add a record in the organic database. CASE mchoice $=1$ 
DO testmemo WITH mchoice, checkba, checkbe, checkr, $0,0,0$

DO testmem2 WITH mchoj.ce

* Display appropriate heading to add/edit organic database

IF addopt $=$.T. ELSE

(1,24 SAY "ADD A RFCORD TO ORGANIC DATABASE" COLOR GR+/B

ENDIF

(@ 3,4 SAY "POLLUTANT:" GET m.pollitant VALID lcase(@m.pollutant)

(1) 4,4 SAY "CAS \#:" GET m.cas num VALID lcase (@m.cas num)

(1) 5,4 SAY "CLASS \# :" GET m.pōll num VALID lcase (@m.p̄oll num)

@ 6,4 SAY "MOLECULAR WEIGHT (amu) : " GET m.mol_wt FUNCTIŌN "@Z" PICTURE

"\#\#\#\#\#\#\#.\#\#\#"

(9 7,4 SAY "BOILING POINT (C): " GET m.boil_pt FUNCTION "@Z" PICTURE

"\#\#\#\#\#\#.\#\#\#"

@ 8,4 SAY "MELTING POINT (C): " GET m.melt_pt FUNCTION "@Z" PICTURE

"\#\#\#\#\#\#.\#\#\#"

(@ 9,4 SAY "VAPOR PRESSURE (Pa):" GET m.vapor pres FUNCTION "@Z" PICTURE "\#\#\#\#\#\#\#\#.\#\#"

@ 10,4 SAY "HENRY'S CONSTANT (Pa-m^3/mol):" GET m.henry_con FUNCTION "@Z" PICTURE "\#\#\#.\#\#\#\#\#\#"

( 11,4 SAY "SOLUBILITY (mg/L):" GET m.solubility FUNCTION "@Z" PICTURE

"\#\#\#\#.\#\#\#\#\#\#\#\#\#\#\#\#\#\#" 12,4 SAY "SOLUBILITY PH:" GET m.sol ph FUNCTION "@Z" PICTURE "\#\#.\#\#"

@ 13,4 SAY "LOG OCTONOL TO WATER PARTITION COEFF:" GET m.log_oct_h2 FUNCTION

"@Z" PICTURE "\#\#\#\#\#\#.\#\#\#\#\#"

(@ 14,4 SAY "IT CAN PHOTODEGRADE (T/F):" GET m.photodeg

@ 15,4 SAY "IT CAN BIODEGRADE (T/F):" GET m.biodegrad

(a) 16,4 SAY "IT IS POLAR (T/F): " GET m.polar

(Q 18,17 SAY "(Check desired box for further information.) " COLOR GR+/B

* Draw a box around check box choices.

(a) 19,4 TO 21,72

* Create check boxes for memo fields.

@ 20,6 GET m. checkba PICTURE '@*C BIOLOGICAL AGENTS';

VALID dispmemo ('bio_agents', addopt)

( 20,32 . GET m. checkbe-PICTURE ' @*C BIOLOGICAL EFFECT';

VALID dispmemo ('bio effect', addopt)

@ 20,58 GET m.checkr PICTURE ' @*C REFERENCE' ;

VALID dispmemo ('reference', addopt)

(a) 22,31 GET okCancel FUNCTION ' $\star_{H}$ SAVE; CANCEL' COLOR SCHEME 1

(@ 17,4 SAY" (Check desired button for further information.)" COLOR GR+/B

(a) 18,4 TO 22,35

(19) 19,6 GET whatmemo FUNCTION;

$\star$ VN BIOLOGICAL AGENTS; BIOLOGICAL EFFECT; REFERENCE' ;

VALID dismemo (1, whatmemo, addopt)

* Create push buttons to save the record or exit the screen.

(a) 22,55 GET okCancel FUNCTION ' ${ }^{\circ} \mathrm{H}$ SAVE; CANCEL' COLOR SCHEME 1

READ CYCLE

* Setup screen to edit/add a record in the inorganic database.

CASE mchoice $=2$

* Display appropriate heading to add/edit inorganic database

IF addopt $=$.T.

(ब) 1,21 SAY "ADD A RECORD TO INORGANIC DATABASE" COLOR GR+/B

ELSE

(1, 1,21 SAY "EDIT A RECORD IN INORGANIC DATABASE" COLOR GR+/B

ENDIF 3,4 SAY "POLLUTANT:" GET m.pollutant VALID lcase(@m.pollutant)

@ 3,4 SAY "POLLUTANT:" GET m. POI VALID lcase (@m.cas_num)

@ 5,4 SAY "CLASS \#:" GET m.pōll num VALID I case(@m.poll_num)

@ 6,4 SAY "VALENCE OF POLLUTANT:" GET m.redox_num;

VALID Icase (@m. redox num)

@ 7,4 SAY "MOLECULAR WEIGHT (amu):" GET m.mol_wt FUNCTION "@Z" PICTURE

"\#\#\#\#\#\#\#\#.\#\#\#"

( 8,4 SAY "BOILING POINT (C):" GET m.boil_pt FUNCTION "@Z" PICTURE

"\#\#\#\#\#\#\#\#" 
(a 9,4 SAY "MELTING POINT (C):" GET m.melt_pt FUNCTION "@Z" PICTURE "\#\#\#\#\#.\#\#\#"

(10,4 SAY "DENSITY $(\mathrm{g} / \mathrm{L}):$ : GET m.density FUNCTION "@Z" PICTURE "\#\#\#\#\#.\#\#\# (1) 11,4 SAY "SOLUBILITY (mg/L):" GET m. solubility FUNCTION "@Z" PICTURE "\#\#\#\# . \#\#\#\#\#\#\#\#\#\#\#\#\#\#"

(1) 12,4 SAY "SOLUBILITY PH:" GET m.sol ph FUNCTION "@Z" PICTURE "\#\#.\#\#" (1) 13,4 SAY "DIFFUSION $\left(\mathrm{cm}^{*} 2 / \mathrm{sec}\right)$ : "GET m.diffusion FUNCTION "@Z" PICTURE "\#\#\#\#\#\#.\#\#\#"

(1) 14,4 SAY "VAPOR PRESSURE (Pa):" GET m.vapor_pres FUNCTION "@Z" PICTURE "\#\#\#\#\#\#\#\#.\#\#"

(@ 15,4 SAY "HENRY'S CONSTANT (Pa-m^3/mol):" GET m.henrY_con FUNCTION "@Z" PICTURE "\#\#.\#\#\#\#\#\#\#"

(18) 18,16 SAY "(Check desired box for further information.)" COLOR GR+/B

* Draw a box around check box choices.

(19) 19,16 TO 21,59

* Create check boxes for memo fields.

( 20,18 GET m.check PICTURE '@*C BIOLOGICAL EFFECT';

VALID dispmemo ('bio effect', addopt)

(1) 20,45 GET m.check PICTURE ' @*C REFERENCE';

VALID dispmemo ('reference', addopt)

* Create push buttons to save the record or exit the screen.

(4) 22,30 GET okCancel FUNCTION $1 *$ H SAVE; CANCEL' COLOR SCHEME 1

READ CYCLE

* Setup screen to edit/add a record in the inor_rad database.

CASE mchoice = 3

$\star$ Display appropriate heading to add/edit radionuclide database

IF addopt $=. T$. ELSE

(a) 0,19 SAY "ADD A RECORD TO RADIONUCLIDE DATABASE" COLOR GR+/B

@ 0,19 SAY "EDIT A RECORD IN RADIONUCLIDE DATABASE" COLOR GR+/B ENDIF

@ 2,2 SAY "POLLUTANT:" GET m.pollutant VALID lcase(@m.pollutant)

@ 3,2 SAY "CAS \#:" GET m.cas_num VALID 1 case(@m.cas_num)

@ 4,2 SAY "CLASS \#: " GET m.pōII num VALID lcase(@m.poll_num)

@ 4,42 SAY "VALENCE OF POLLUTANT:" GET m.redox_num; VALID Icase (@m. redox num)

@ 5,2 SAY "MOLECULAR WEIGHT (amu):" GET m.mol_wt FUNCTION "@Z" PICTURE

"\#\#\#\#\#\#\#\#.\#\#\#"

(@) 5,42 SAY "DENSITY ( $/ \mathrm{L}):$ : GET m.density FUNCTION "@Z" PICTURE "\#\#\#\#\#.\#\#" @ 6,2 SAY "BOILING POINT (c) : " GET m.boil_pt FUNCTION "@Z" PICTURE "\#\#\#\#\#\#.\#\#"

@ 6,42 SAY "MELTING POINT (C):" GET m.melt_pt FUNCTION "@Z" PICTURE "\#\#\#\#\#\#.\#\#\#"

(@ 7,2 SAY "DIFFUSION ( $\left.\mathrm{cm}^{\wedge} 2 / \mathrm{sec}\right): "$ GET m.diffusion FUNCTION "@Z" PICTURE "\#\#\#\#\#\#.\#\#\#"

@ 7,42 SAY "VAPOR PRESSURE (Pa):" GET m.vapor_pres FUNCTION "@Z" PICTURE "\#\#\#\#\#\#\#\#.\#\#"

(9) 8,2 SAY "HALF LIFE (yrs): " GET m.half_life FUNCTION "@Z" PICTURE

"\#\#\#\#\#\#\#\#\#\#\#.\#\#\#\#\#\#\#\#"

@ 8,42 SAY "DAUGHTER:" GET m.daughter VALID l case (@m.daughter)

@ 9,2 SAY "SOLUBILITY (mg/L): " GET m. solubility FUNCTION "@Z" PICTURE "\#\#\#\#.\#\#\#\#\#\#\#\#\#\#\#\#\#\#\#"

@ 9,42 SAY "SOLUBILITY PH: " GET m. sol ph FUNCTION "@Z" PICTURE "\#\#.\#\#"

@ 10,2 SAY "HENRY'S CONSTANT $\left(\mathrm{Pa}-\mathrm{m}^{\wedge} 3 / \mathrm{mol}\right):$ " GET m.henry_con FUNCTION "@Z"

PICTURE "\#\#.\#\#\#\#\#\#\#\#"

(a) 12,2 SAY "(Check all types of emission.)" COLOR GR+/B

* Draw a box and create emission checkboxes.

(1) 13,3 TO 17,14

(14,18 SAY "EMISSION 1:"

(4) 14,4 GET m.picalph PICTURE '@*C ALPHA'; VALID place (14,30,picalph, 'emission_1')

(1) 15,18 SAY "EMISSION 2:"

@ 15,4 GET m.picbeta PICTURE '@*C BETA'; VALID place (15,30,picbeta,'emission_2')

(16 16,18 SAY "EMISSION 3:" 
(9) 16,4 GET m.picgamm PICTURE ' @*C GAMMA';

VALID place $(16,30$, picgamm, 'emission $31 ;$

(9) 14,42 SAY "ENERGY 1 (mev): " GET m.eñergY_1 FUNCTION "@Z" PICTURE "\#\#\#\#\#.\#\#"

@ 15,42 SAY "ENERGY 2 (mev):" GET m.energY_2 FUNCTION "@Z" PICTURE

"\#\#\#\#\#.\#\#"

@ 16,4 ? SAY "ENERGY 3 (mev) :" GET m.energY_3 FUNCTION "@Z" PICTURE

"\#\#\#\#\# .\#\#"

* Draw a box around check box choices.

(a) 18,16 SAY "(Check desired box for further information.) " COLOR GR+/B

(4) 19,16 TO 21,60

* Create check boxes for memo fields.

( 120,18 GET m.check PICTURE '@*C BIOLOGICAL EFFECT';

VALID dispmemo ('bio effect', addopt)

@ 20,46 GET m.check PICTURE ' @*C REFERENCE';

VALID dispmemo ('reference', addopt)

* Create push buttons to save the record or exit the screen.

@ 22,31 GET okCancel FUNCTION ' $*$ H SAVE; CANCEL' COLOR SCHEME 1

READ CYCLE

* Setup screen to edit/add a record in the technology database.

CASE mchoice $=4$

* Display appropriate heading to add/edit technology database

IF addopt $=$. T.

ELSE

@ 0,20 SAY "ADD A RECORD TO TECHNOLOGY DATABASE" COLOR GR+/B

ENDIF

@ 0,20 SAY "EDIT A RECORD IN TECHNOLOGY DATABASE" COLOR GR+/B

@ 2,4 SAY "TECHNOLOGY:" GET m.technology VALID lcase (@m.technology)

(a) 3,4 SAY "SUB-CLASS:" GET m. sub class VALID lcase (@m.sub_class)

(1) 4, 12 SAY "(Choose the correct Eechnology group and domain.)" COLOR GR+/B

( 5,4 SAY "GENERAL TECHNOLOGY GROUP:"

(1) 5,50 SAY "BIOLOGICAL TYPE:" COLOR B/BG

(1) 5,67 SAY m.tech_oxi

( 6,6 GET m.pusht $\bar{g}$ FUNCTION;

' $\star$ HN BIOLOGICAL ; THERMAL; IMMOBILIZATION ; PHYSICAL/CHEMICAL ; UNKNOWN' ;

VALID push $(5,30$, pushtg, 'tech_group')

(1) 7,4 SAY "DOMAIN:"

@ 8,12 GET m.pushd FUNCTION '*HN IN-SITU;EX-SITU;UNKNOWN';

VALID push $(7,12$, pushd, 'domain')

(a) 9,4 SAY "MEDIA:"

(9,11 SAY "(Check all applicable media.)" COLOR GR+/B

(10) 10,25 SAY 'MEDIA $1:{ }^{\prime}$

(1) 11,25 SAY 'MEDIA 2:'

(1) 12,25 SAY 'MEDIA $3: '$

(1) 10,12 GET m.Picks PICTURE '@*C SOIL';

VALID place $(10,34$, picks, 'medial')

( 11,12 GET m.pickw PICTURE '@*C WATER';

VALID place (11,34, pickw, 'media2')

@ 12,12 GET m.picka PICTURE '@*C AIR';

VALID place (12,34,picka, 'media3')

(1) 13,4 SAY "REMEDIATION PHASE:"

@ 13,23 SAY "(Check all appropriate remediation phases.)" COLOR GR+/B

(1) 14,36 SAY "REM. PHASE 1:"

(1) 15,36 SAY "REM. PHASE 2:"

(16, 16 SAY "REM. PHASE $3: "$

(3) 17,36 SAY "REM. PHASE 4:"

(14,12 GET m.PickC PICTURE '@*C CHARACTERIZATION';

VALID place $(14,50$, pickc, 'rem phasel')

@ 15,12 GET m.picke PICTURE ' @ *C EXTRACTION';

VALID place $(15,50$, picke, 'rem phase2')

(16) 16,12 GET m.pickt PICTURE '@‡C TREATMENT';

VALID place $(16,50$, pickt, 'rem phase 3')

(1) 17,12 GET m.pickd PICTURE ' ब*C DISPOSAL';

VALID place $(17,50$, pickd, 'rem phase4')

* Draw a box around check box choices. 
(18,16 SAY "(Check desired box for further information.)" COLOR GR+/B

(19) 19,4 TO 21,74

* Create check boxes for memo fields.

(1) 20,6 GET m.check PICTURE '@*C DESCRIPTION';

VALID dispmemo('descript', addopt)

(1) 20,31 GET m.check PICTURE ' @*C REFERENCE' ;

VALID dispmemo ('reference', addopt)

@ 20,55 GET $m$. check PICTURE ' @*C POLLUTANT INFO';

VALID addextra ('ypollut',m.poll key)

* Create push buttons to save the-record or exit the screen.

(a) 22,31 GET okCancel FUNCTION $1{ }^{*} \mathrm{H}$ SAVE; CANCEL' COLOR SCHEME 1

READ CYCLE

* Setup screen to edit/add a record in the develop database.

CASE mchoice $=5$

* This DO WHILE loop allows the user to go back and forth between

* two screens when in the develop database.

DO WHILE continue

DO CASE

CASE screen $=1$

* Define and activate the first screen of the develop record.

DEFINE WINDOW record FROM 0,0 TO 24,79 COLOR SCHEME 5

ACTIVATE WINDOW record

* Define help popup

DEFINE MENU thelp2 IN WINDOW record

DEFINE PAD helppad OF thelp2 PROMPT 'HELP' AT 0,70

ON PAD helppad OF fhelp2 ACTIVATE POPUP help2

DEFINE POPUP help2 FROM 1,67 IN WINDOW record COLOR SCHEME 1

DEFINE BAR 1. OF help2 PROMPT 'Field Definitions' COLOR SCHEME 10

DEFINE BAR 2 OF help2 PROMPT 'Cancel' COLOR SCHEME 10

ON SELECTION POPUP help2 DO helpit WITH BAR(),2

ACTIVATE MENU fhelp2 NOWAIT

* Write the current contents of the specified field on the

* screen above the push buttons.

(1) 9,39 SAY m.tech group

(1) 17,12 SAY m.domāin

(a) 9, il SAY m.scale

* Display appropriate heading to add/edit develop database

IF addopt $=$.T.

(a) 0,16 SAY "ADD A RECORD TO DEVELOP DATABASE (Screen 1)" COLOR GR+/B ELSE

(Q) 0,15 SAY "EDIT A RECORD IN DEVELOP DATABASE (Screen 1)" COLOR $\mathrm{GR}+/ \mathrm{B}$

ENDIF

(a) 2,4 SAY "COMPANY'S NAME FOR TECHNOLOGY:"

(1) 3,16 GET m.tech name VALID lcase(@m.tech_name)

@ 4,4 SAY "TECHNOLOGY:" GET m. technology;

VALID Icase (@m.technology)

@ 5,4 SAY "DEVEIOPER:" GET m.developer VALID lcase(@m.developer)

( 6, 4 SAY "SITE:" GET m.site VALID lcase(@m.site)

(1) 7,10 SAY;

"(Choose the correct scale, domain, and technology group.)" COLOR $\mathrm{GR}+/ \mathrm{B}$

@ 9,4 SAY "SCALE:"

* Draw a box around scale push buttons and define choices.

(a) 10,6 TO 16,16

(1) 11,7 GET m.pushsc FUNCTION 1 *VN BENCH; LAB; PILOT;FIELD;UNKNOWN ' ; VALID push $(9,11$, pushsc, 'scale')

@ 17,4 SAY "DOMAIN:"

* Draw a box around domain push buttons and define choices.

(1) 18,6 TO 22,16

( 19,7 GET m.pushd FUNCTION '*VN IN-SITU;EX-SITU;UNKNOWN' ; 
VALID push $(17,12$, pushd, 'domain')

(a) 9,21 SAY "TECHNOLOGY GROUP:"

* Draw a box around tech group push buttons and define choices.

(1) 10,23 TO 16,46

(a) 11,24 GET m.pushtg FUNCTION;

' *VN BIOLOGICAL ; THERMAL; IMMOB ILIZATION ; PHYSICAL/CHEMICAL ; UNKNOWN ' ;

VALID push $(9,39$, pushtg, 'tech group')

(1) 11,50 SAY "BIOLOGICAL TYPE:" COLOR B/BG

@ 11,67 SAY m.tech_oxi

* Enable user to continue to the next screen.

(8) 22,60 GET change1 PICTURE ' @*CT NEXT SCREEN' COLOR $\ldots \ldots \ldots, \ldots / B G$

* Create push buttons to save the record or exit the screen.

(Q) 22,30 GET okcancel PICTURE '@ \$H SAVE;CANCEL' COLOR SCHEME 1

READ CYCLE

* If the "NEXT SCREEN" checkbox is chosen, change the

* value of the screen to 2 (second screen), and

* reinitialize the value of the checkbox to 0 .

IF change $1=1$

screen $=2$

ENDIF

change $1=0$

CASE screen $=2$

check $=0$

$\star$ Define and activate the second screen of the develop record.

DEFINE WINDOW devscreen FROM 0,0 TO 24,79 COLOR SCHEME 5

ACTIVATE WINDOW devscreen

* Define help popup for second develop screen

DEFINE MENU thelp3 IN WINDOW devscreen

DEFINE PAD helppad OF thelp3 PROMPT 'HELP' AT 0,70

ON PAD helppad OF fhelp3 ACTIVATE POPUP help3

DEFINE POPUP help3 FROM 1,67 IN WINDOW devSCreen COLOR SCHEME 1

DEFINE BAR 1 OF help3 PROMPT 'Fields Definitions' COLOR SCHEME 10

DEFINE BAR 2 OF help3 PROMPT 'Cancel' COLOR SCHEME 10

ON SELECTION POPUP help3 DO helpit WITH BAR(),3

ACTIVATE MENU fhelp3 NOWAIT

* Write the current contents of the specified field on the

* screen beside the checkboxes.

IF m.medial = 'soil'

m.picks $=1$

(a) 5,34 SAY m.medial

ENDIF

IF m.media2 = 'water'

$\mathrm{m} \cdot \mathrm{pickw}=1$

(9) 6,34 SAY m.media2

ENDIF

IF m.media3 = 'air'

m. picka $=1$

(a) 7,34 SAY m.media3

ENDIF

IF $m$.rem phasel = 'characterization'

m.pick $\bar{c}=1$

(1) 11,50 SAY m.rem phasel

ENDIF

IF m.rem_phase2 = 'extraction'

m. pick $\bar{e}=1$

(a) 12,50 SAY m.rem phase2

ENDIF

IF $m$.rem phase 3 = 'treatment'

m. pick $\bar{E}=1$

(1) 13,50 SAY m.rem_phase3

ENDIF

IF $m$.rem_phase4 ='disposal' 
$m \cdot$ pickd $=1$

@ 14,50 SAY m.rem_phase4

ENDIF

* Display appropriate heading to add/edit develop database

IF addopt $=$. T . (8) 0,16 SAY "ADD A RECORD TO DEVELOP DATABASE (Screen 2)" COLOR GR $+/ E$ ELSE

@ 0,15 SAY "EDIT A RECORD IN DEVELOP DATABASE (Screen 2)" COLOR $G R+/ B$

ENDIF

(@ 3,4 SAY "MEDIA:"

@ 3,11 SAY "(Check all applicable media.)" COLOR GR+/B

(1) 5,25 SAY 'MEDIA 1:'

Q 6,25 SAY 'MEDIA $2: 1$

(1) 7,25 SAY 'MEDIA $3: 1$

(1) 5,12 GET m.picks PICTURE '@*C SOIL';

VALID place $(5,34$, picks, 'medial')

(1) 6,12 GET m.PICkW PICTURE ' $@ *$ C WATER';

VALID place $(6,34$, pickw, 'media2')

(1) 7,12 GET m.picka PICTURE '@*C AIR'; VALID place $(7,34$, picka, 'media3')

(a) 9,4 SAY "REMEDIATION PHASE:"

@ 9,23 SAY "(Check all appropriate remediation phases.)" COLOR GR+/B

(1) 11,36 SAY "REM. PHASE 1:"

(Q 12,36 SAY "REM. PHASE 2:"

(13) 13,36 SAY "REM. PHASE 3:"

(14) 14,36 SAY "REM. PHASE 4:"

(1) 11,12 GET m.PICKC PICTURE '@*C CHARACTERIZATION';

VALID place $(11,50$, pickc, 'rem phase1')

(12,12 GET m.picke PICTURE ' @ ॠC EXTRACTION';

VALID place ( 12,50 , picke, 'rem phase2')

(1) 13,12 GET m.pickt PICTURE '@ ॠC TREATMENT';

VALID place $(13,50$, pickt, 'rem phase 3')

(14 14,12 GET m.Pickd PICTURE ' @ \#C DISPOSAL';

VALID place (14,50, pickd, 'rem phase4')

* Draw a box around check box choices.

(1) 17,17 SÂ" "(Check desired box for further information.) " COLOR GR+/B

(@ 18,4 TO 21,73

* Create check boxes for memo fields.

@ 19,5 GET m.check PICTURE ' @*C DESCRIPTION'; VALID dispmemo ('descript', addopt)

( 20,5 GET m.check PICTURE '@*C DOCUMENTS';

VALID dispmemo ('documents', addopt)

(a) 19,24 GET m.check PICTURE '@*C STATUS';

VALID dispmemo ('status', addopt)

(a) 20,24 GET m.check PICTURE '@*C COST';

VALID dispmemo ('cost', addopt)

(1) 19,38 GET $m$. check PICTURE ' $₫ * C$ CONTACTS';

VALID dispmemo ('contacts', addopt)

@ 20,38 GET m. check PICTURE ' @*C REFERENCE';

VALID dispmemo ('reference', addopt)

( 19,55 GET m.check PICTURE ' @ ${ }^{\circ}$ MEDIA INFO';

VALID addextra ('ymedia',m.media key)

( 20,55 GET m.check PICTURE '@*C DOLLUTANT INFO';

VALID addextra ('ypollut', m.poll key)

( 22,54 GET change 2 PICTURE $@$ @ *CT PREVIOUS SCREEN

* Create push buttons to save or exit the screen.

( 22,30 GET OkCancel FUNCTION '*H SAVE;CANCEL' COLOR SCHEME 1

READ CYCLE

* If the "PREVIOUS SCREEN" checkbox is chosen, change the

* value of the screen to 1 (first screen), and

* reinitialize the value of the checkbox to 0.

IF change $2=1$

screen $=1$ 


\section{ENDCASE}

\section{ENDIF}

change $2=0$

* If at any time in either screen SAVE or CANCEL are chosen,

* exit the DO WHILE loop and continue the program.

IF (okcancel = 1). OR. (okcancel $=2$ ) EXIT

ENDIF

ENDDO

ENDCASE

* Pass control depending on which push button is chosen

* If SAVE is chosen, transfer the memory variables to the database IF okcancel = 1

IF (appblank = .F.). AND. (addopt = .T.)

APPEND BLANK

ENDIF

GATHER MEMVAR

ELSE

* If SAVE is chosen and then CANCEL, only save the values given

* to media key and poll key.

IF sacancel $\equiv 1$

DO CASE

CASE mChoice $=4$

GATHER MEMVAR FIELDS poll_key

CASE MChOICE $=5$

ENDCASE

GATHER MEMVAR FIELDS media_key,poll_key

ENDIF

ENDIF

* If CANCEL is chosen or after SAVE choice is executed,

* deactivate window and pass control

DEACTIVATE WINDOW record

DEACTIVATE WINDOW devscreen

* If in add mode, pass control to mainmenu

IF addopt $=$. $T$.

CLOSE DATABASES

RETURN

* If in edit mode, pass control to editmenu

ELSE

* When returning control to editmenu clear the searchstring. srchstring $=$ SFACE $(30)$ RETURN

ENDIF

* This function can be used with any of the fields where there are specific choices given in push buttons.

* This function will display the choice made on the screen.

FUNCTION push

PARAMETERS $x, y$, push, fldname

* $x$ and $y$ are the coordinates where the choice will be written.

* Push determines which button was pushed.

* Fldname is the name of the field which is being edited.

* When a button is pushed, determine which field is being used.

DO CASE

* Assign a value to the field domain depending on which

* button was chosen.

CASE fldname = 'domain'

DO CASE

CASE push $=1$

m. domain $=$ 'in-situ' 
CASE push $=2$

m.domain $=$ 'ex-situ'

CASE push $=3$

ENDCASE

m. domain $=$

Assign a value to the field tech_group depending on which

button was chosen.

CASE Eldname = 'tech group'

DO CASE

CASE push $=1$

m.tech group = 'biological

* If biological is chosen, display a window to ask which

* type of technology it is.

DO CASE

* Place the window at certain coordinates depending on

* whether you are in technology or develop database.

CASE mchoice $=4$ DEFINE WINDOW trY FROM 8,42 TO 13,79 COLOR SCHEME 8

CASE mChoice $=5$

ENDCASE

DEFINE WINDOW trY FROM 14,42 TO 19,79 COLOR SCHEME 8

ACTIVATE WINDOW trY

(a) 0,1 SAY "Choose biological technology type:" COLOR B/BG

Q 1,13 GET m.bioOpt FUNCTION ' *VT AEROBIC; ANAEROBIC; COMBINED'

READ

* Write the choice into the database under field tech_oxi.

DO CASE

CASE bioopt $=1$ m.tech oxi = 'aerobic

CASE biōpt $=2$

m.tech oxi = 'anaerobic'

CASE bioōpt $=3$

\section{ENDCASE}

m.tech_oxi = 'combined'

DEACTIVATE WINDOW trY

* If any other tech group is chosen, clear the tech_oxi field.

CASE push $=2$

m.tech-group $=$ 'thermal

m.tech oxi =

CASE push $=3$

m.tech_group $=$ 'immobilization

m.tech oxi =

CASE push $=4$

m.tech group = 'physical/chemical'

m. tech-oxi = '

CASE push $=5$

$m$. tech group $=$

m. tech_oxi = '

ENDCASE

* Regardless of the tech group, print the contents of

* tech oxi on the screen beside the BIOLOGICAL TYPE heading.

* The Iocation is different for the technology and develop database.

DO CASE

CASE inchoice $=4$

(1) 5,67 SAY m.tech_oxi

CASE mchoice $=5$

ENDCASE

(a) 11,67 SAY m.tech_oxi

* Assign a value to the field scale depending on which

button was chosen.

CASE Eldname $=$ 'scale'

DO CASE

CASE push $=1$ 


\section{ENDCASE}

m.scale $=$ 'bench'

CASE push $=2$

m. scale $=1 \mathrm{ab}$

CASE push $=3$

m.scale $=$ 'pilot'

CASE push $=4$

m.scale $=$ 'Eield'

CASE push $=5$

\section{ENDCASE}

m.scale $=$ '

* Display the choice made at the specified coordinates.

@ $x, y$ SAY m.\&fldname

* Reassign push to 0 .

push $=0$

RETURN

* This function can be used with any of the fields where there are specific choices given in check boxes.

* This function will confirm that the user has chosen a check box

* by placing the contents of the field on the screen.

FUNCTION place

PARAMETER $x, y, p i c k$, fieldnm

* $x$ and $y$ are the coordinates where the choice will be written.

* Pick determines whether the checkbox was clicked or unclicked.

* Fieldnm is the name of the field which is being edited.

* If a checkbox is unclicked pick $=0$. Assign blanks to the field.

IF pick $=0$

DO CASE

CASE mchoice $=3$

m. \&fieldnm $=$

CASE mchoice $=4$

m.\&fieldnm $=$

CASE mchoice $=5$

ENDCASE m. \&fieldnm $=1$

* If a checkbox is clicked, pick = 1. Assign the choice to the field.

ELSE

DO CASE

CASE fieldnm = 'emission_1'

m.emission 1 = 'alpha'

CASE fieldnm = 'emission 2 '

m.emission $2=$ 'beta'

CASE fieldnm ${ }^{-}=$'emission $3 '$

m.emission $3=$ 'gamma'

CASE fieldnm $=$ 'medial'

m. medial = 'soil

CASE fieldnm = 'media2'

m.media2 = 'water'

CASE fieldnm = 'media3'

m.media $3=$ 'air'

CASE fieldnm = 'rem phase 1 '

m.rem phase 1 = 'characterization'

CASE $\mathrm{fieldnm}=$ ' rem phase 2 '

m. rem phase 2 ='extraction'

CASE fieldnm = 'rem phase 3 '

m. rem phase $3=$ 'treatment'

CASE fieldnm = 'rem phase4'

ENDCASE

m.rem_phase4 = 'disposal'

ENDIF

* Write the new field contents at the designated coordinates. 
@ $x, y$ SAY m.\&fieldnm
$\star$ Reassign pick to 0.
pick $=0$

RETURN

* This function converts all data entry to lower case.

FUNCTION lcase

PARAMETER string

string = LOWER (string)

RETURN

* This procedure calls another program which defines fields

* for the user.

PROCEDURE helpit

PARAMETERS mchoice, whichelp

DO CASE

CASE mchoice $=1$

DO fieldhel IN $c: \backslash$ metc\database \prog\fieldhel.prg WITH mchoice, whichelp

CASE mchoice $=2$

DO CASE

CASE whichelp $=1$

DEACTIVATE POPUP helpl

CASE whichelp $=2$

DEACTIVATE POPUP help2

CASE whichelp $=3$

DEACTIVATE POPUP help3

CASE whichelp $=4$

DEACTIVATE POPUP help4

CASE whichelp $=5$

DEACTIVATE POPUP help5

CASE whichelp $=6$

ENDCASE

DEACTIVATE POPUP help6

\section{ENDCASE}

RETURN

*

* This function creates a region to add/edit memo fields.

FUNCTION dispmemo

PARAMETERS memname, addopt, check

savecan $=0$

IF addopt $=. T$.

appblank $=. \mathrm{T}$.

ENDIF

APPEND BLANK

${ }^{\star} \mathrm{m}$. check $=0$

GATHER MEMVAR

DEFINE WINDOW dispmemo FROM 0,0 TO 25,79 COLOR SCHEME 7

ACTIVATE WINDOW dispmemo

(@) 1,31 SAY 'MEMO REQUESTED'

* This variable will hold the original contents of the memo field

* so that the changes will be erased from the screen if CANCEL

* is chosen.

mvar $=m$. \&memname

* Create a text editing region and push buttons.

(1) 3,2 EDIT m.\&memname SIZE 18,75 SCROLL COLOR SCHEME 10

(1) 22,32 GET savecan FUNCTION $1 *$ H SAVE; CANCEL'

- READ CYCLE

* The SAVE/CANCEL buttons give the user the option to save

* or disregard the changes to the memo.

DO CASE 
* If the user chooses SAVE, the memo data will be gathered.

CASE savecan $=1$

GATHER MEMVAR FIELDS \&memname MEMO

DEACTIVATE WINDOW dispmemo

* DO testmemo WITH mchoice, checkba, checkbe, checkr, 0, 0,0

* Otherwise, the changes to the memo field will be disregarded.

CASE savecan $=2$

* This will clear the changes which were disregarded from

* the screen.

m. \&memname = mvar

ENDCASE

DEACTIVATE WINDOW dispmemo

\section{RETURN}

* This function will allow the user to add/edit pollutant or

* media information.

FUNCTION addextra

PARAMETER medpol1, key

IF addopt $=. T$.

APPEND BLANK

ENDIF

GATHER MEMVAR

DO CASE

CASE medpoll = 'ymedia'

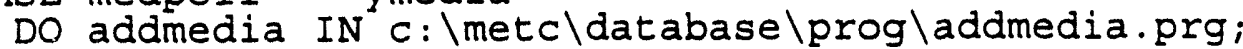
WITH key, sacancel, addopt

CASE medpoll = 'ypollut'

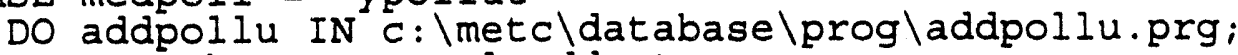

ENDCASE WITH key, sacancel, addopt

RETURN

FUNCTION dismemo

PARAMETERS mchoice, whatmemo, addopt

savecan $=0$

IF addopt $=. T$.

appblank $=. T$.

AEPEND BLANTK

ENDIF

GATHER MEMVAR

DEFINE WINDOW dispmemo FROM 0,0 TO 25,79 COLOR SCHEME 7 ACTIVATE WINDOW dispmemo

@ 1,31 SAY 'MEMO REQUESTED'

DO CASE

CASE mchoice $=1$

DO CASE

CASE whatmemo $=1$

memname $=$ 'bio agents'

CASE whatmemo $=2$

memname $=$ 'bio_effect'

CASE whatmemo $=3$

ENDCASE

memname $=$ 'reference'

\section{ENDCASE}

* Create a text editing region and push buttons.

(a) 3,2 EDIT m. \&memname SIZE 18,75 SCROLL COLOR SCHEME 10

(a) 22,32 GET savecan FUNCTION ' $*$ H SAVE; CANCEL'

READ CYCLE 
* This variable will hold the original contents of the memo field * so that the changes will be erased from the screen if CANCEL

* is chosen.

mvar $=$ m.\&memname

* The SAVE/CANCEL buttons give the user the option to save

* or disregard the changes to the memo.

DO CASE

* If the user chooses SAVE, the memo data will be gathered.

CASE savecan $=1$

GATHER MEMVAR FIELDS \&memname MEMO

DEACTIVATE WINDOW dispmemo

DO testmem2 WITH mchoice

* Otherwise, the changes to the memo field will be disregarded.

CASE savecan $=2$

* This will clear the changes which were disregarded from

* the screen.

m. \&memname = mvar

DEACTIVATE WINDOW dispmemo

ENDCASE

RETURN 
* PROCEDURE TO DELETE A RECORD

PROCEDURE delrec

PARAMETERS mchoice

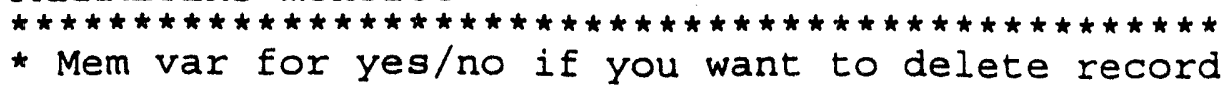

yesno $=1$

$\operatorname{delm}=0$

delp $=0$

delitp $=. T$.

delitm $=. T$.

DEFINE WINDOW delete FROM 7,31 TO 14,49 PANEL SHADOW;

COLOR SCHEME 8

DEFINE WINDOW really FROM 7,28 TO 14,53 PANEL SHADOW;

COLOR SCHEME 1

ACTIVATE WINDOW delete

* Get user input yes/no

(1) 1,2 SAY "ARE YOU SURE?"

@ 3,3 GET Yesno FUNCTION '*H YES;NO'

READ CYCLE

* If the YES button is chosen ...

$I F$ yesno $=1$

* Check again to make sure user wants to delete record

DEACTIVATE WINDOW delete

ACTIVATE WINDOW really

* Get user confirmation

(1) 1,2 SAY "ARE YOU REALLY SURE?"

@ 3,7 GET Yesno FUNCTION $1 * \mathrm{H}$ YES;NO'

READ CYCLE

* If user chooses yes delete record; otherwise return

* to edit menu

* If the YES button is chosen again, delete the record.

IF yesno $=1$

DO CASE

CASE mchoice $=4$

IF poll key $=0$

$\operatorname{delit} \bar{p}=. F$.

ELSE

delp = poll_key

ENDIF

CASE mchoice $=5$

IF poll_key $=0$

delit $\bar{p}=. F$.

ELSE delp = poll_key

ENDIF

IF media key $=0$

delitm ${ }^{-}=$.F.

ELSE

delm = media_key

ENDCASE

ENDIF

DELETE

PACK

IF delitp $=. T$.

SELECT 8

USE C: \METC \DATABASE\DATA \POLLUTANT.DBF

SET ORDER TO TAG POII_kEY

SEEK delp

DELETE

ENDIF

PACK

IF delitm $=. T$.

SELECT 9

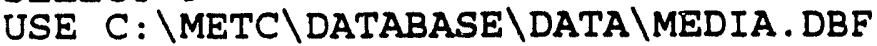




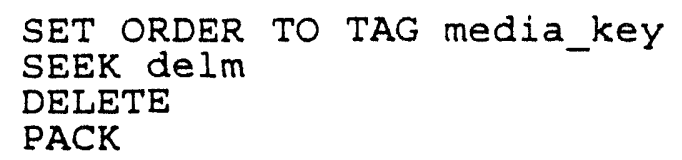




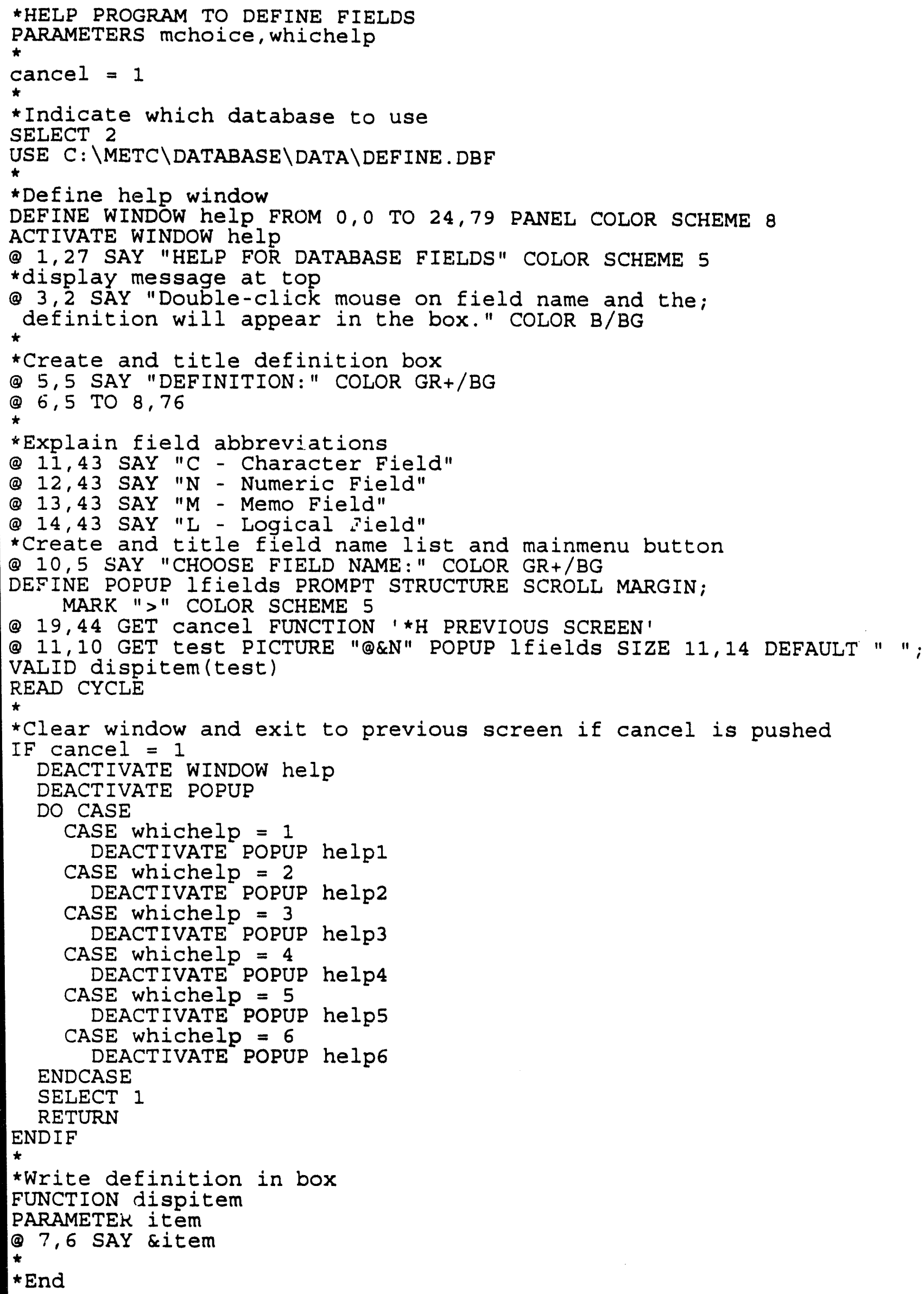




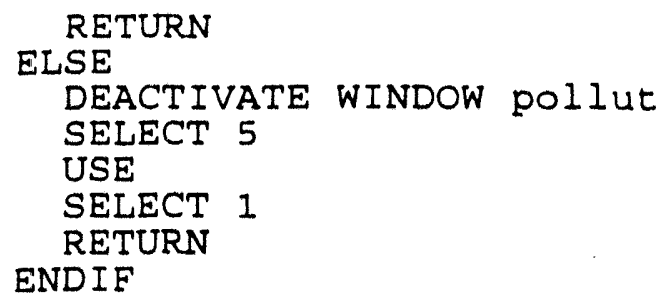




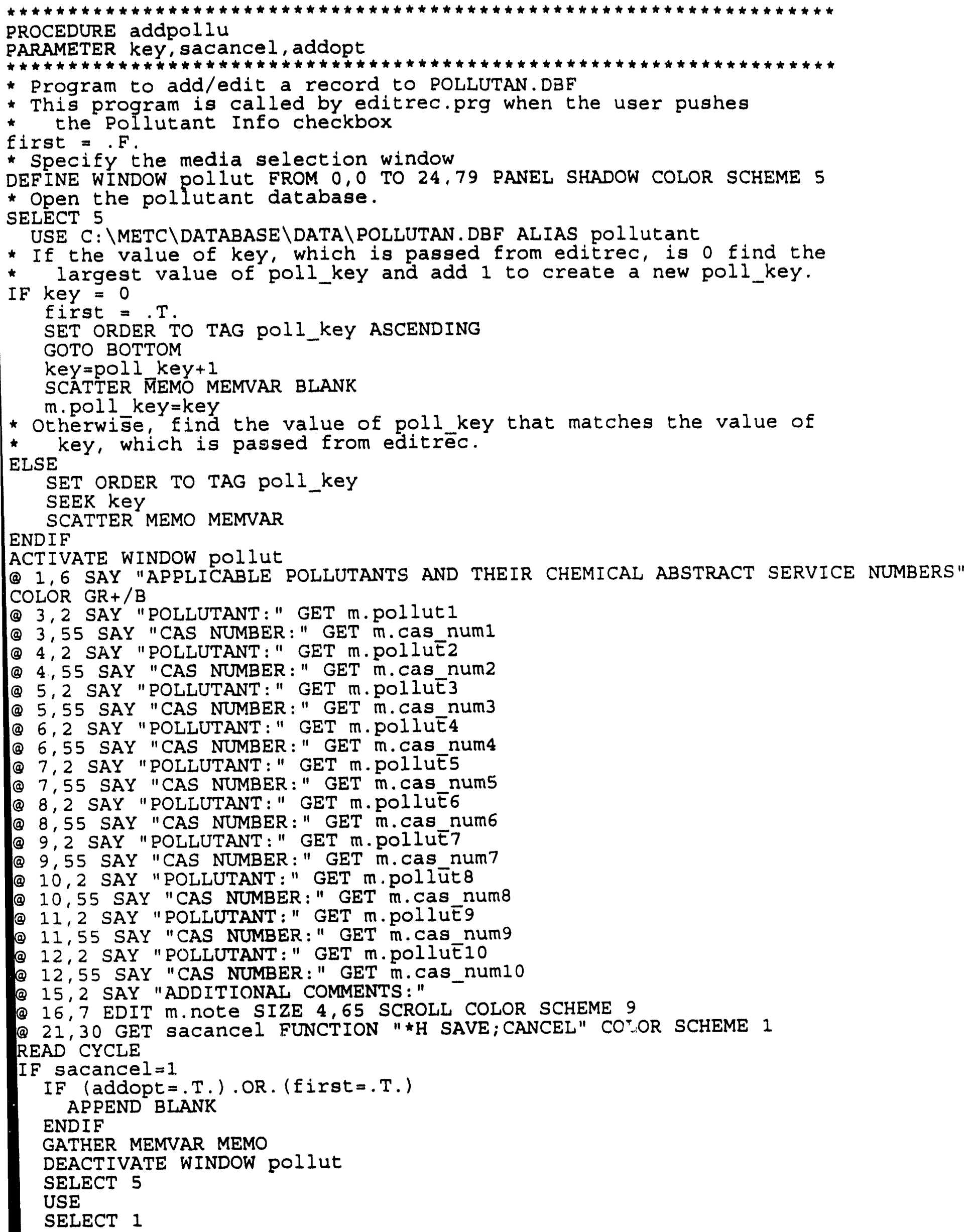


* screen in addmedia.

IF sacancel=1

* Put "air" into media3.

m. mediathree= "air"

IF (addopt $=$.T. .). OR. (Eirst $=$. T.)

APPEND BLANK

ENDIF

GATHER MEMVAR

DEACTIVATE WINDOW addair

SELECT 4

USE

SELECT 1

RETURN

* Otherwise, return to the first screen in addmedia.

ELSE

* IF CANCEL is chosen, reassign the field contents to

* their original value which was held in the

* memory variables.

m.a pressure = mapress

$\mathrm{m} \cdot \mathrm{a}^{-}$flow $=$maflow

m. $a^{-}$temp $=$matemp

m.a_humidity = mahum

DEACTIVATE WINDOW addair

SELECT 4

USE

SELECT 1

RETURN

ENDIF

ENDCASE

* If the PREVIOUS RECORD button has been chosen, return to the calling

* program(addmedia), and then return to the last screen displayed

* in editrec.

ELSE

DEACTIVATE WINDOW mediasel

SELECT 4

USE

SELECT 1

RETURN

ENDIF

* Display new choice for popup selection on popup

FUNCTION schoice

SHOW GETS

RETURN 
* Otherwise, return to the first screen in addmedia.

ELSE

* IF CANCEL is chosen, reassign the field contents to

* their original value which was held in the

* memory variables.

m. w org cont = mworg

$\mathrm{m} \cdot \mathrm{w}^{-}$colloid $=$mwcoll

$m \cdot w_{-O X Y}$ cont $=$ mwoxy

$m \cdot w$ depEh $=$ mwdepth

$m \cdot w-E l O w=m w f l O w$

$m \cdot w^{-}$temp $=$mwtemp

$\mathrm{m} \cdot \mathrm{w}_{-} \mathrm{ph}=\mathrm{mwph}$

\section{DEACTIVATE WINDOW addwater}

SELECT 4

USE

SELECT 1

RETURN

ENDIE

* If medianair is selected

CASE mediabut $=3$

DEACTIVATE WINDOW mediasel

* Specify the air window

DEFINE WINDOW addair FROM 0,0 TO 24,79 COLOR SCHEME 5

ACTIVATE WINDOW addair

* Store original field contents in memory variables so that the

* changes will be erased from the screen if CANCEL is chosen. mapress $=m \cdot a$ pressure

maflow $=m \cdot a$ Elow

matemp $=m \cdot a^{-}$temp

mahum $=m \cdot a_{-}$humidity

* Define help popup

DEFINE MENU thelp6 IN WINDOW addair

DEFINE PAD helppad OF fhelp6 PROMPT 'HELP' AT 0,70

ON PAD helppad OF fhelp6 ACTIVATE POPUP help6

DEFINE POPUP help6 FROM 1,67 IN WINDOW record COLOR SCHEME 1

DEFINE BAR 1 OF help6 PROMPT 'Field Definitions' COLOR SCHEME 10

DEFINE BAR 2 OF help6 PROMPT 'Cancel' COLOR SCHEME 10

ON SELECTION POPUP help6 DO helpit WITH BAR(), 6

ACTIVATE MENU fhelp6 NOWAIT

* Mem var for save/cancel

sacancel $=0$

* Store air data to variables

IF addopt $=. T$.

ELSE

(1) 1,26 SAY "ADD AIR MEDIA INFORMATION" COLOR GR+/B

ENDIF

@ 1,26 SAY "EDIT AIR MEDIA INFORMATION" COLOR GR+/B

@ 3,4 SAY "MEDIA \#3: AIR"

@ 4,4 SAY "AIR ABSOLUTE PRESSURE(Pa):" GET m.a_pressure FUNCTION "@Z"

PICTURE "\#\#\#\#\#\#.\#\#\#"

(9) 5,4 SAY "AIR VOLUMETRIC FLOW RATE $\left(m^{\wedge} 3 / \mathrm{hr}\right)$ : " GET m.a_flow FUNCTION "@Z"

PICTURE "\#\#\#\#\#\#.\#\#\#"

(1) 6,4 SAY "AIR TEMPERATURE(deg C):" GET m.a_temp FUNCTION "@Z" PICTURE "\#\#\#.\#\#"

@ 7,4 SAY "AIR HUMIDITY $(\xi):$ " GET m.a humidity FUNCTION "@Z" PICTURE "\#\#\#.\#\#"

(1) 11,21 SAY "(Check box for further information.)" COLOR GR+/B

(a) 12,23 TO 14,54

@ 13,24 GET m.check PICTURE "@*C AIR ADDITIONAL INFORMATION";

VALIn dispmemo ('a comment', addopt)

(1) 19,31 GET sacanceI FUNCTION ' $\star H$ SAVE; CANCEL' COLOR SCHEME 1

READ CYCLE

* If save is chosen, save the record and return to the first 
DEFINE WINDOW addwater FROM 0,0 TO 24,79 COLOR SCHEME 5 ACTIVATE WINDOW addwater

* Store original field contents in memory variables so that the

* changes will be erased from the screen if CANCEL is chosen. mworg $=m \cdot w$ org cont

mwCOIL $=m \cdot \bar{w}$ coIloid

mwoxy $=m \cdot w_{-}$oxy_cont

mwdepth $=m \cdot$. w dēpth

MWElOW $=m \cdot w$ ElOW

mwtemp $=m \cdot w$ temp

$\mathrm{mwph}=\mathrm{m} \cdot \mathrm{w}$. $\mathrm{ph}$

* Define help popup

DEFINE MENU fhelp5 IN WINDOW addwater

DEFINE PAD helppad OF fhelp5 PROMPT 'HELP' AT 0,70

ON PAD helppad OF thelp5 ACTIVATE POPUP help5

DEFINE POPUP help5 FROM 1,67 IN WINDOW record COLOR SCHEME 1

DEFINE BAR 1 OF help5 PROMPT 'Field Definitions' COLOR SCHEME 10

DEFINE BAR 2 OF help5 PROMPT 'Cancel' COLOR SCHEME 10

ON SELECTION POPUP help5 DO helpit WITH BAR(), 5

ACTIVATE MENU Ehelp5 NOWAIT

* Mem var for save/cancel

sacancel $=0$

IF addopt $=$. T.

(a) 1,25 SAY "ADD WATER MEDIA INFORMATION" COLOR GR+/B

ELSE

(Q 1,25 SAY "EDIT WATER MEDIA INFORMATION" COLOR GR+/B

ENDIF

(1) 3,4 SAY "MEDIA \#2: WATER"

@ 4,4 SAY "WATER ORGANIC CONTENT(mg/L):" GET m.w_org_cont FUNCTION "@Z"

PICTURE "\#\#.\#\#"

(\$) 5,4 SAY "WATER COLLOIDAL CONTENT (mg/L):" GET m.w_colloid FUNCTION "@Z" PICTURE "\#\#\#\#.\#\#\#\#\#\#\#\#\#\#"

( 6, 4 SAY "DISSOIVED OXYGEN CONTENT(mg/L):" GET m.w_OXY_cont FUNCTION "@Z"

PICTURE "\#\#.\#\#"

@ 7,4 SAY "DEPTH TO WATER TABLE $(\mathrm{m}):$ " GET m.w_depth FUNCTION "@Z" PICTURE

"\#\#\#.\#\#\#"

@ 8,4 SAY "VOLUMETRIC FLOW RATE $\left(\mathrm{m}^{\wedge} 3 / \mathrm{hr}\right): "$ GET m.w_flow FUNCTION "@Z" PICTURE

@\#\#\#\#\#.\#\#\#" 9,4 SAY "WATER TEMPERATURE (deg C) : " GET m.w_temp FUNCTION "@Z" PICTURE

"\#\#\#.\#\#"

@ 10,4 SAY "WATER pH:" GET m.w_ph FUNCTION "@Z" PICTURE "\#\#.\#\#"

@ 13,21 SAY "(Check box for further information.) " COLOR GR+/B

(14) 14,22 TO 17,55

(15,23 GET m.check PICTURE "@*C WATER BIOTA DESCRIPTION";

VALID dispmemo ('w_biota', addopt)

(1) 16,23 GET m.check-PICTURE "Q*C WATER ADDITIONAL INFORMATION";

VALID dispmemo ('w comment', addopt)

( 20,31 GET sacanceI FUNCTION ' ${ }^{\circ}$ H SAVE; CANCEL' COLOR SCHEME 1

READ CYCLE

* If save is chosen, save the record and return to the first

* screen in addmedia.

IF sacancel $=1$

* Put "water" into media2.

m. mediatwo = "water"

IF $($ addopt $=$. T.). OR. (Eirst.- I. $)$

APPEND BLANK

ENDIF

GATHER MEMVAR

DEACTIVATE WINDOW addwater

SELECT 4

USE

SELECT 1

RETURN 

"\#\#.\#\#" "13,4 SAY "SOIL TEMPERATURE (deg C):" GET m.s_temp FUNCTION "@Z" PICTURE @ 14,4 SAY "BULK DENSITY $\left(\mathrm{kg} / \mathrm{m}^{\wedge} 3\right):$ " GET m.s_bulk_den FUNCTION "@Z" PICTURE

@ 15,4 SAY "CATION EXCHANGE CAPACITY(meq/g):" GET m.s_cat_exch FUNCTION "@Z" PICTURE "\#\#\#\#\#\#.\#\#\#"

(17,21 SAY "(Check box for further information.)" COLOR GR+/B

(4) 18,22 TO 21,55

(@ 19,23 GET m. check PICTURE "@*C SOIL BIOTA DESCRIPTION";

VALID dispmemo ('s_biota', addopt)

(8) 20,23 GET m.check-PICTURE "Q*C SOIL ADDITIONAL INFORMATION"; VALID dispmemo ('s_comment', addopt)

@ 5,55 GET SOiltext-PICTURE "@ " UNKNOWN ;CLAY;SILTY CLAY;SANDY CLAY; ;

CLAY LOAM; SIILTY CLAY LOAM; SANDY CLAY LOAM; SANDY LOAM; SILT LOAM; LOAM; ;

LOAMY SAND; SAND; SILT" WHEN schoice()

* Create push buttons to save or exit the screen.

(a) 22,31 GET sacancel FUNCTION $1 *$ H SAVE; CANCEL' COLOR SCHEME 1

READ CYCLE

* Selection from Popup is copied into s_texture

m.s texture =LOWER (soiltext)

* IE save is chosen, save the record and return to the first

* screen in addmedia.

IF sacancel $=1$

* Put "soil" into medial.

m. mediaone $=$ "soil"

IF $($ addopt $=. T.) \cdot O R$. (first $=. T$.

APPEND BLANK

ENDIF

GATHER MEMVAR

DEACTIVATE WINDOW addsoil

SELECT 4

USE

SELECT 1

RETURN

* Otherwise, return to the first screen in addmedia.

ELSE

* IF CANCEL is chosen, reassign the field contents to

* their original value which was held in the

* memory variables.

m.s_texture = mstext

m.s_sand_per = msand

m.s_silt_per = msilt

m.s_clay_per = mclay

$\mathrm{m} \cdot \mathrm{s}$-ph $=-\mathrm{msph}$

m.s_hydr_con = mshydr

m.s sorg cont $=$ msorg

$\mathrm{m} \cdot \mathrm{s}$ h2o-cont $=\mathrm{msh} 20$

$\mathrm{m}$. $\mathrm{s}$ volume $=\mathrm{msvol}$

m.s temp = mstemp

$\mathrm{m} . \mathrm{s}$ bulk den $=\mathrm{msbulk}$

$\mathrm{m} \cdot \mathrm{s}_{\text {_cat_exch }}=\mathrm{mscat}$

DEACTIVATE WINDOW addsoil

SELECT 4

USE

SELECT 1

RETURN

ENDIF

* If media=water is selected

CASE mediabut $=2$

DEACTIVATE WINDOW mediasel

* Specify the water window 
- If mediasosil is selected CASE mediabut $=1$

DEACTIVATE WINDOW mediasel

* Specify the soil window

ACTIVATE WINDOW addsoil

* Store original field contents in memory variables so that the

* changes will be erased from the screen if CANCEL is chosen. mstext $=$ m.s texture

msand $=m \cdot s$ sand_per

msilt $=m \cdot s^{-}$silt per

mclay $=$ m.s.clay_per

$\mathrm{msph}=\mathrm{m} \cdot \mathrm{s} \overline{\mathrm{p} h}$

mshydr $=$ m.s hydr_con

msorg $=\mathrm{m}$.s örg cönt

msh $20=m \cdot s$ h $20^{-c o n t}$

msvol = m.s volüme

mstemp $=m \cdot \bar{s}$ temp

msbulk = m.s bulk den

mscat $=$ m.s $\bar{c} a t$ exch

* Mem var for save/cancel

sacancel $=0$

* Initialize POPUP variable

soiltext=SPACE (15)

* If the value of s_texture is blank, have UNKNOWN appear in

* th popup.

IF m.s texture =

STORE "UNKNOWN "TO soiltext

* Otherwise, have the value it has been given appear in the popup.

ELSE

upcase = UPPER (m.s texture)

STORE upcase TO soİlext

ENDIF

* Define help popup

DEFINE MENU Ehelp4 IN WINDOW addsoil

DEFINE PAD helppad OF fhelp4 PROMPT 'HELP' AT 0,70

ON PAD helppad OF thelp4 ACTIVATE POPUP help4

DEFINE POPUP help4 FROM 1,67 IN WINDOW record COLOR SCHEME 1

DEFINE BAR 1 OF help4 PROMPT 'Field Definitions' COLOR SCHEME 10

DEFINE BAR 2 OF help4 PROMPT 'Cancel' COLOR SCHEME 10

ON SEIECTION POPUP help4 DO helpit WITH BAR(), 4

ACTIVATE MENU Ehelp4 NOWAIT

IF addopt $=$.T.

(a) 1,26 SAY "ADD SOIL MEDIA INFORMATION" COLOR GR+/B

ELSE

@ 1,26 SAY "EDIT SOIL MEDIA INFORMATION" COLOR GR+/B

ENDIF

@ 3,4 SAY "MEDIA \#1: SOIL"

* Caption over POPUP box

( 4,55 SAY "CHOOSE SOIL TEXTURE:" COLOR B/BG

* Selection from POPUP is placed next to "SOIL TEXTURE: "

(1) 4,4 SAY "SOIL TEXTURE:" GET soiltext

(9) 5,4 SAY "PERCENT SAND $(z):$ " GET m.s_sand_per FUNCTION "@Z" PICTURE "\#\#.\#\#"

(@ 6,4 SAY "PERCENT SILT $(\gamma):$ " GET m.s_silt_per FUNCTION "@Z" PICTURE

"\#\#.\#\#"

( 7,4 SAY "PERCENT CLAY $(q):$ " GET m.s_clay_per FUNCTION "@Z" PICTURE

"\#\#.\#\#"

@ 8,4 SAY "SOIL pH:" GET m.s ph FUNCTION "@Z" PICTURE "\#\#.\#\#"

(1) 9,4 SAY "HYDRAULIC CONDUCTIVITY $(\mathrm{cm} / \mathrm{s})$ : " GET m.s_hydr_con FUNCTION "@Z" PICTURE "\#\#\#\#\#\#.\#\#\#\#\#\#\#\#\#\#"

@ 10,4 SAY "ORGANIC CONTENT( $(\xi)$ : GET m.s_org_cont FUNCTION "@Z" PICTURE "\#\#.\#\#"

(1) 11,4 SAY "MOISTURE CONTENT(\%):" GET m.s_h2o_cont FUNCTION "@Z" PICTURE 


\section{PROCEDURE addmedia}

PARAMETER key, sacancel, addopt

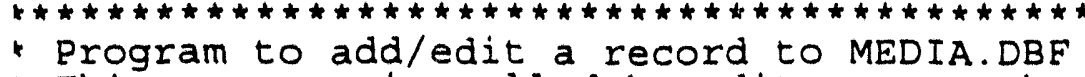

- This program is called by editrec.prg when the user pushes the Media Info checkbox

nedloop $=. T$.

irst $=$. F.

- Mem var for continue/previous record and media selection buttons :ontpr $=0$

nediabut $=1$

- Specify the media selection window.

JEFINE WINDOW mediasel FROM 0,0 TO 24,79 COLOR SCHEME 5

- Open the media database.

¿ELECT 4

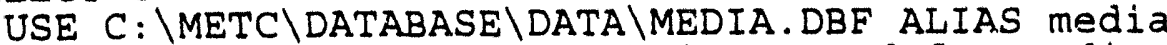

- If the value of key, which is passed from editrec, is 0 find the

- largest value of media_key and add 1 to create a new media_key.

[F key $=0$

Eirst $=$. T.

SET ORDER TO TAG media_keY ASCENDING

GOTO BOTTOM

key =media key+1

SCATTER MEMO MEMVAR BLANK

m. media key $=$ key

- Otherwise, find the value of media key that matches the value of LSE key, which is passed from editrec.

SET ORDER TO TAG media_key

SEEK KEY

SCATTER MEMO MEMVAR

NDIF

This do while loop allows the user to move back and forth between the two screens in addmedia.

DO WHILE medloop

ELECT 4

USE C: $\backslash$ METC $\backslash$ DATABASE \DATA \MEDIA.DBE ALIAS media

CTIVATE WINDOW mediasel

5,5 SAY "SELECT A MEDIA TYPE IN ORDER TO SUPPLY DETAILED MEDIA INFORMATION" OLOR GR+/B

8,28 TO 12,46 COLOR SCHEME 1

9,30 GET mediabut FUNCTION $1 * R$ SOIL MEDIA; WATER MEDIA;AIR MEDIA'

Define set of pushbuttons to save/cancel

@ 14,15 SAY "Push CONTINUE to add media data; push PREVIOUS RECORD to exit" 15,23 GET ContPr FUNCTION $1{ }^{H}$ CONTINUE; PREVIOUS RECORD';

VALID edmedia(contpr, mediabut) COLOR SCHEME 1

EAD CYCLE

If the PREVIOUS RECORD button is chosen, exit the do while loop

and return to the last screen displayed in editrec.

$F$ contpr $=2$

EXIT

NDIF

NDDO

ETURN

This function is the second screen in addmedia.

UNCTION edmedia

ARAMETERS contpr, mediabut, changeback

EFINE WINDOW addsoil FROM 0,0 TO 24,79 COLOR SCHEME 5

If the CONTINUE button is chosen, display the second screen

in addmedia.

heck $=0$

F contpr $=1$

DO CASE 
* Mem var for edit/delete/previous menu

editdel = 0

printit $=0$

DEFINE WINDOW display FROM 0,0 TO 24,79 COLOR SCHEME 1

* This do while loop allows the user to go back and forth between

* the displayed record and either the first screen in editrec or

the first screen in printrpt.

DO WHILE contin

ACTIVATE WINDOW display

DO CASE

* Display organic pollutant record

CASE mchoice $=1$

(a) 0,23 SAY "RECORD FROM ORGANIC DATABASE"

(1) 3,4 SAY "POLLUTANT:" GET pollutant DISABLE

@ 4,4 SAY "CAS \#:" GET cas_num DISABLE

(a) 5,4 SAY "CLASS \#:" GET pōll num DISABLE

@ 6,4 SAY "MOLECULAR WEIGHT (ämu): " GET mol wt FUNCTION "@Z" DISABLE

@ 7,4 SAY "BOILING POINT (C): " GET boIl pt FUNCTION "@Z" DISABLE

@ 8,4 SAY "MELTING POINT (C):" GET melt_pt FUNCTION "@Z" DISABLE

@ 9,4 SAY "VAPOR PRESSURE (Pa):" GET vap̃or pres FUNCTION "@Z" DISABLE

(1) 10,4 SAY "HENRY'S CONSTANT (Pa-m^3/mol): "GET henrY_COn FUNCTION "@Z"

DISABLE

@ 11,4 SAY "SOLUBILITY (mg/L):" GET solubility FUNCTION "@Z" DISABLE

(12,4 SAY "SOLUBILITY PH:" GET sol ph FUNCTION "@Z" DISABLE

(1) 13,4 SAY "LOG OCTONOL TO WATER PARTITION COEFF:" GET' IOg_OCt_h2 FUNCTION "@Z" DISABLE

(1) 14,4 SAY "IT CAN PHOTODEGRADE (T/E):" GET photodeg DISABLE

(1) 15,4 SAY "IT CAN BIODEGRADE (T/F):" GET biodegrad DISABLE

( ) 16,4 SAY "IT IS POLAR (T/F):" GET polar DISABLE

* Define set of push buttons to edit/delete/previous menu

(Q) 20,22 GET editdel EUNCTION '*H EDIT;DELETE; PREVIOUS MENU'

(9) 22,30 GET printit FUNCTION '*H PRINT RECORD' COLOR SCHEME 7

READ CYCLE

* Display inorganic pollutant record

CASE mchoice $=2$

@ 0,22 SAY "RECORD FROM INORGANIC DATABASE"

@ 3,4 SAY "POLLUTANT:" GET pollutant DISABLE

(4) 4, 4 SAY "CAS \#:" GET cas_num DISABLE

@ 5,4 SAY "CLASS \#:" GET pŌII num DISABLE

(1) 6,4 SAY "VALENCE OF POLLUTANTT:" GET redoX_num DISABLE

@ 7,4 SAY "MOLECULAR WEIGHT (amu): " GET moI-wt EUNCTION "@Z" DISABLE

@ 8,4 SAY "BOILING POINT (C): ".GET boil pt FUNCTION "@Z". DISABLE

@ 9,4 SAY "MELTING POINT (C):" "GET melt_pt FUNCTION "@Z" DISABLE

@ 10,4 SAY "DENSITY (g/L):" GET density FUNCTION "@Z" DISABLE

@ 11,4 SAY "SOLUBILITY (mg/L): " GET solubility FUNCTION "@Z" DISABLE

@ 12,4 SAY "SOLUBILITY PH:" GET sol ph FUNCTION "@Z" DISABLE

(a) 13,4 SAY "DIFFUSION (cmi2/sec): " GET diffusion FUNCTION "@Z" DISABLE

@ 14,4 SAY "VAPOR PRESSURE (Pa):" GET vapor pres FUNCTION "@Z" DISABLE

@ 15,4 SAY "HENRY's CONSTANT (Pa-m^3/mol):" GET henrY_CON FUNCTION "@Z"

DISABLE

* Define set of push buttons to edit/delete/previous menu

(1) 20,22 GET editdel FUNCTION $1 \star^{*}$ EDIT;DELETE; PREVIOUS MENU'

@ 22,30 GET printit FUNCTION $1 *$ H PRINT RECORD' COLOR SCHEME 7

READ CYCLE

* Display radionuclide record

CASE mchoice $=3$

( ) 0,21 SAY "RECORD FROM RADIONUCLIDE DATABASE"

@ 2,4 SAY "POLLUTANT:" GET pollutant DISABLE

@ 3,4 SAY "CAS \#:" GET CaB_num DISABLE 
( 4,4 SAY "CLASS \#:" GET poll nUm DISABLE

@ 5,4 SAY "VALENCE OF POLLUTANTT:" GET redOX num DISABLE

(a) 6,4 SAY "MOLECULAR WEIGHT (amu): " GET mol-wt FUNCTION "@Z" DISABLE

(a 7,4 SAY "BOILING POINT (C):" GET boil pt FUNCTION "@Z" DISABLE

(a) 8,4 SAY "MELTING POINT (C):" GET melt pt FUNCTION "@Z" DISABLE

(1) 9,4 SAY "DENSITY $(\mathrm{g} / \mathrm{L})$ : " GET density FUNCTION "@Z" DISABLE

@ 10,4 SAY "SOLUBILITY (mg/L): " GET SOlubility FUNCTION "@Z" DISABLE

@ 11,4 SAY "SOLUBILITY PH:" GET SOI Ph FUNCTION "@Z" DISABLE

@ 12,4 SAY "DIFFUSION $\left(\mathrm{cm}^{*} 2 / \mathrm{sec}\right):$ " GET diffusion FUNCTION "@Z" DISABLE

@ 13,4 SAY "VAPOR PRESSURE (Pa):" GET vapor_pres FUNCTION "@Z" DISABLE

@ 14,4 SAY "HENRY'S CONSTANT $\left(\mathrm{Pa}-\mathrm{m}^{\wedge} 3 / \mathrm{mol}\right):$ "GET henry_con FUNCTION "@Z"

\section{DISABLE}

@ 15,4 SAY

(16) 16,4 SAY

@ 17,4 SAY

"HALF LIFE (yrs):" GET half life FUNCTION "@Z" DISABLE

"DAUGHTER:" GET daughter DIS̄ABLE

(8) 18,4 SAY

"EMISSION 1:" GET emission 1 DISABLE

(a) 19,4 SAY

"EMISSION 2:" GET emission-2 DISABLE

@ 17,30 SAY

"EMISSION 3:" GET emission-3 DISABLE

@ 18,30 SAY "ENERGY 2 (mev) :" GET energY_2 FUNCTION "@Z" DISABLE

"ENERGY 1 (mev) : " GET energy_ I FUNCTION "@Z" DISABLE

* Define set of push buttons to edit/delete/previous menu

@ 22,22 GET editdel FUNCTION $1 *$ H EDIT;DELETE; PREVIOUS MENU'

@ 22,62 GET printit FUNCTION $1 *$ H PRINT RECORD' COLOR SCHEME 7

READ CYCLE

* Display technology record

CASE mchoice $=4$

(Q) 0,21 SAY "RECORD FROM TECHNOLOGY DATABASE"

(1) 3,4 SAY "TECHNOLOGY:" GET technolOgY DISABLE

(a) 4,4 SAY "SUB-CLASS:" GET sub Class DISABLE

@ 5,4 SAY "TECHNOLOGY GROUP:" GET tech_group DISABLE

@ 6,7 SAY "OXIDATION TYPE (for biological technologies only):" GET tech_oxi

DISABLE

@ 7,4 SAY "DOMAIN:" GET domain DISABLE

@ 8,4 SAY "MEDIA I:" GET medial DISABLE

@ 9,4 SAY "MEDIA 2:" GET media2 DISABLE

(10,4 SAY "MEDIA 3 : " GET media3 DISABLE

@ 11,4 SAY "REMEDIATION PHASE 1:" GET rem_phaseI DISABLE

@ 12,4 SAY "REMEDIATION PHASE 2:" GET rem_phase2 DISABLE

@ 13,4 SAY "REMEDIATION PHASE 3:" GET rem_phase3 DISABLE

@ 14,4 SAY "REMEDIATION PHASE 4:" GET rem_phase4 DISABLE

* Define set of push buttons to edit/deleEe/previous menu

@ 20,22 GET editdel FUNCTION $1 \star_{\mathrm{H}}$ EDIT;DELETE; PREVIOUS MENU'

@ 22,30 GET printit FUNCTION $1 *$ H PRINT RECORD' COLOR SCHEME 7

READ CYCLE

* Display develop record

CASE mchoice $=5$

(a) 0,22 SAY "RECORD FROM DEVELOP DATABASE"

@ 3,4 SAY "COMPANY'S NAME FOR TECHNOLOGY:" GET tech_name DISABLE

( 4,4 SAY "TECHNOLOGY:" GET techNOIOgY DISABLE

(@) 5,4 SAY "DEVELOPER:" GET developer DISABLE

@ 6,4 SAY "GENERAL TECHNOLOGY GROUP:" GET tech_group DISABLE

@ 7,7 SAY "OXIDATION TYPE (for biological techñologies only): " GET tech_oxi

\section{DISABLE}

(Q) 8,4 SAY "SITE:" GET site DISABLE

(a) 9,4 SAY "DOMAIN:" GET doma in DISABLE

(10) 10,4 SAY "SCALE:" GET scale DISABLE

(a) 11,4 SAY "MEDIA 1:" GET medial DISABLE

(a) 12,4 SAY "MEDIA $2: "$ GET media2 DISABLE

(a) 13,4 SAY "MEDIA 3:" GET media3 DISABLE

(14) 14,4 SAY "REMEDIATION PHASE 1:" GET rem_phase1 DISABLE

@ 15,4 SAY "REMEDIATION PHASE 2:" GET rem_phase2 DISABLE

(16,4 SAY "REMEDIATION PHASE 3:" GET rem_phase3 DISABLE

@ 17,4 SAY "REMEDIATION PHASE 4:" GET rem_phase4 DISABLE

* Define set of push buttons to edit/delete/previous menu

(1) 20,22 GET editdel FUNCTION $1 *$ H EDIT;DELETE; PREVIOUS MENU'

(1) 22,30 GET printit FUNCTION $1 *$ H PRINT RECORD' COLOR SCHEME 7 


\section{READ CYCLE}

ENDCASE

* Control passes depending on which push button is chosen

DO CASE

* If edit is chosen, control is passed to editrec.

CASE editdel $=1$

DEACTIVATE WINDOW display

DO editrec WITH mchoice, F., recnum IN $C: \backslash M E T C \backslash D A T A B A S E \backslash P R O G \backslash E D I T R E C$. PRG editdel = 0

* If delete is chosen, control is passed to delrec

CASE editdel $=2$

DEACTIVATE WINDOW display

DO delrec WITH mchoice IN $C: \backslash M E T C \backslash D A T A B A S E \backslash P R O G \backslash D E L R E C$. PRG

* If previous menu is chosen, control is passed to editmenu.

CASE editdel = 3

DEACTIVATE WINDOW display

\section{CLOSE DATABASES}

* When returning control to editmenu clear the searchstring. srchstring = SPACE (30)

ENDCASE

* If the PRINT RECORD button is chosen, pass control to the

* printrpt program.

IF printit $=1$

DO C: \METC \DATABASE \PROG \PRINTRPT.PRG WITH mchoice, recnum printit $=0$

ANDIF

$\because$ If the DELETE or PREVIOUS MENU buttons are chosen, exit the do while loop and return to editmenu.

$F($ editdel $=2)$. OR. (editdel $=3$ )

EXIT

:NDIF

iNDDO

:ETURN 
PROCEDURE printrpt

PARAMETERS mchoice, recnum

DEFINE WINDOW ChOices FROM 0,0 TO 24,79 PANEL SHADOW COLOR SCHEME 1 ACTIVATE WINDOW choices

SET TALK OFF

prnchoice $=1$

@ 5,13 SAY"This option allows you to print the record as a report!"

(1) 10,20 SAY"Please select a printing option."

(8) 12,32 GET Prnchoice FUNCTION $1 * \mathrm{~V}$ PRINTER; SCREEN ; CANCEL '

READ CYCLE

DO CASE

CASE prnchoice $=1$

DO printer WITH mchoice, recnum

CASE prnchoice $=2$

DO screen WITH mchoice, recnum

CASE prnchoice $=3$

DEACTIVATE WINDOW choices

ENDCASE

RETURN

*

*

PROCEDURE printer

PARAMETERS mChoice, recnum

STORE RECNO() TO recnum

* This memory variable remains true unless media_key $=0$. getm $=. T$.

* This memory variable remains true unless poll_key $=0$. getp $=. T$.

DO CASE

CASE mchoice $=1$

REPORT FORM C: $\backslash$ METC $\backslash D A T A B A S E \backslash R P T \backslash O R G A N I C . F R X$ TO PRINT RECORD reCnUM NOEJECT

CASE mChoice $=2$

REPORT FORM C: $\backslash$ METC $\backslash D A T A B A S E \backslash R P T \backslash I N O R G A N . F R X$ TO PRINT RECORD recnum NOEJECT

CASE mChoice $=3$

REPORT FORM C: \METC \DATABASE \RPT \RADIONUC.FRX TO PRINT RECORD recnUm NOEJECT

CASE mChOice $=4$

REPORT FORM C: $\backslash$ METC \DATABASE\RPT \TECHRPT.FRX TO PRINT RECORD recnUm NOEJECT

*** ALSO PRINT POLLUTANT INFORMATION ***

* Reassign memory variable to .F. if there is no pollutant info.

IF poll_key =0 getp $\equiv . F$.

* Otherwise, assign the poll_key number to a seek variable.

ELSE

ENDIF

whichp $=$ poll_key

* If there is pollutant information, open pollutan.dbf and

* seek the corresponding record.

IF getp $=. T$.

SELECT 8

USE C $: \backslash M E T C \backslash D A T A B A S E \backslash D A T A \backslash P O L L U T A N$. DBF

SET ORDER TO TAG POII_keY

SEEK whichp

STORE RECNO() TO pOIlute

REPORT FORM C: \METC\DATABASE\RPT\POLLUTE.FRX TO PRINT RECORD pollute NOEJECT

* Close the pollutant database.

SELECT 8

USE 
ENDIF

* Return to the initial work area.

SELECT 1

CASE mchoice $=5$

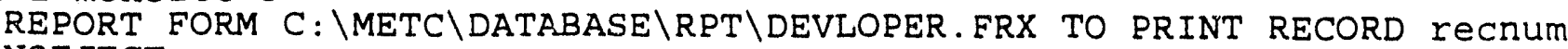
NOEJECT

* Re ALsO PRINT MEDIA INFORMATION $\star * \star$
Reassign memory variable to . F. if there is no media info.

IF media_key $=0$ getm $=-$. $F$.

* Otherwise, assign the media_key number to a seek variable.

ELSE

ENDIF

* If there is media information, open media.dbf and seek

* the corresponding record.

IF getm $=. T$.

SELECT 9

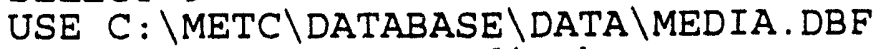

SET ORDER TO TAG media_key

SEEK whichm

STORE RECNO() TO media

* If soil information is provided, print the soil report.

IF mediaone = 'soil'

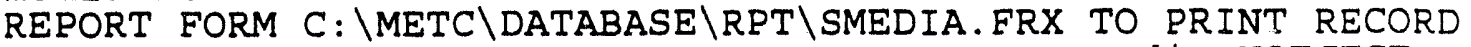

ENDIF

* If water information is provided, print the water report.

IF mediatwo = 'water'

REPORT FORM C: \METC \DATABASE \RPT \WMEDIA.FRX TO PRINT RECORD ENDIF media NOEJECT

* If air information is provided, print the air report.

IF mediathree = 'air'

REPORT FORM C: \METC \DATABASE\RPT \AMEDIA.FRX TO PRINT RECORD ENDIF media NOEJECT

* Close the media database.

SELECT 9

USE

* Return to the initial work area.

FNDIF

SELECT 1

*** ALSO PRINT POLLUTANT INFORMATION * * *

* Reassign memory variable to .F. if there is no pollutant info.

IF poli key $=0$ getp $\equiv . F$.

* Ocherwise, assign the poll_key number to a seek variable. ELSE

whichp $=$ poll_key

ENDIF

* If there is pollutant information, open pollutan.dbf and seek the corresponding record.

IF getp $=. T$.

SELECT 8

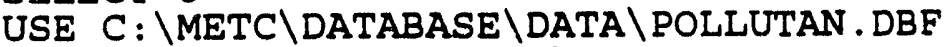

SET ORDER TO TAG pOII_keY

SEEK whichp

STORE RECNO() TO pollute

REPORT FORM C: \METC\DATABASE\RPT\POLLUTE.FRX TO PRINT RECORD 
pollute NOEJECT

* Close the pollutant database.

SELECT 8

USE

ENDIF

turn to the initial work area.

SELECT 1

ENDCASE

DEACTIVATE WINDOW choices

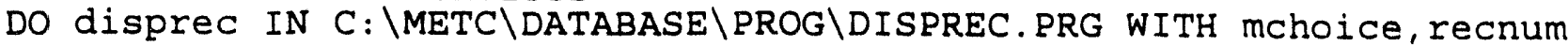
$\star$

PROCEDURE screen

PARAMETERS mchoice, recnum

STORE RECNO() TO recnum

SET TALK OFF

* This memory variable remains true unless media_key $=0$.

jetm $=. \mathrm{T}$.

* This memory variable remains true unless poll_key $=0$.

jetp $=. T$.

DO CASE

CASE mchoice $=1$

REPORT FORM C: \METC\DATABASE\RPT\ORGANIC.FRX RECORD recnUm PREVIEW

CASE mChOice $=2$

REPORT FORM C: $\backslash M E T C \backslash D A T A B A S E \backslash R P T \backslash I N O R G A N . F R X$ RECORD recnUm PREVIEW

CASE MChOice $=3$

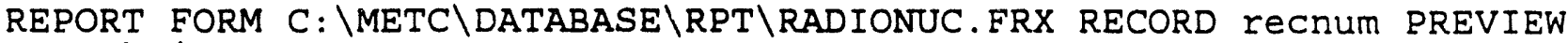

CASE mChOice $=4$

REPORT FORM $C: \backslash M E T C \backslash D A T A B A S E \backslash R P T \backslash T E C H R P T . F R X$ RECORD recnUM PREVIEW

* * * ALSO PRINT POLLUTANT INFORMATION $\star * *$

* Reassign memory variable to .F. if there is no pollutant info.

IF poll key = 0 getp $\equiv . F$.

* Otherwise, assign the poll_key number to a seek variable.

ELSE

ENDIF

whichp $=$ poll key

* If there is pollutant information, open pollutan.dbf and

* seek the corresponding record.

IF getp $=. T$.

SELECT 8

USE C : \METC \DATABASE\DATA \POLLUTAN.DBE

SET ORDER TO TAG pOII keY

SEEK whichp

STORE RECNO() TO pollute

REPORT FORM C: \METC\DATABASE\RPT\POLLUTE.FRX RECORD pollute PREVIEW

* Close the pollutant database.

SELECT 8

USE

* Return to the initial work area.

ENDIF SELECT 1

CASE mchoice $=5$

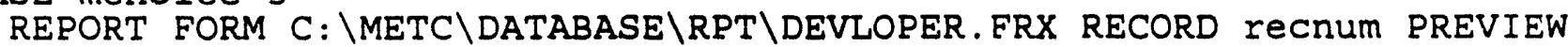

* * * ALSO PRINT MEDIA INFORMATION * * *

* Reassign memory variable to .F. if there is no media info.

IF media key $=0$

getm $=-$. F.

* otherwise, assign the media_key number to a seek variable. ELSE 
ENDIF

whichm = media_key

* If there is media information, open media.dbf and seek

* the corresponding record.

IF getm $=. T$.

SELECT 9

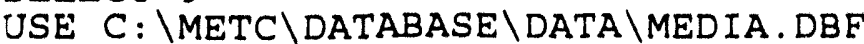

SET ORDER TO TAG media_keY

SEEK whichm

STORE RECNO() TO media

* If soil information is provided, print the soil report.

IF mediaone = 'soil' ENDIF

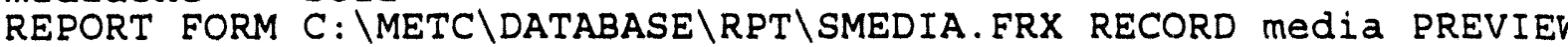

* If water information is provided, print the water report.

IF mediatwo = 'water'

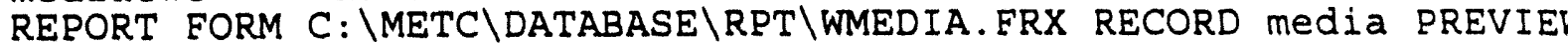
ENDIF

* If air information is provided, print the air report.

IF mediathree = 'air'

REPORT FORM $C: \backslash M E T C \backslash D A T A B A S E \backslash R P T \backslash A M E D I A . F R X$ RECORD media PREVIEV ENDIF

* Close the media database.

SELECT 9

USE

* Return to the initial work area.

ENDIF

SELECT 1

$\star \star \star$
* Reassign memory variable to .F. if there is no pollutant info.

IF poli_key $=0$

getp $\equiv . F$.

* Otherwise, assign the poll_key number to a seek variable.

ELSE ENDIF

whichp = poll_key

* If there is pollutant information, open pollutan.dbf and

* seek the corresponding record.

IF getp $=. T$.

SELECT 8

USE C: $\backslash$ METC \DATABASE \DATA \POLLUTAN. DBF

SET ORDER TO TAG pOII_keY

SEEK whichp

STORE RECNO() TO pollute

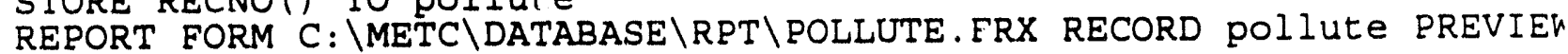

* Close the pollutant database.

SELECT 8

USE

* Return to the initial work area.

ENDIF

SELECT 1

NDCASE

JEACTIVATE WINDOW choices

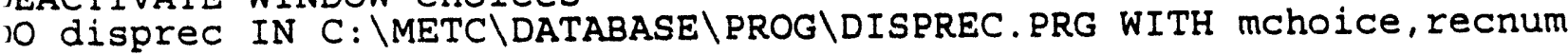




\section{CLOSE DATABASES}

\section{SET TAIK OFF}

\section{CLEAR}

SELECT 1

USE $c: \backslash$ metc $\backslash$ database \data\organ_ch.dbf

SELECT 2

USE $c: \backslash$ metc $\backslash$ database \data\chem_cla.dbf

SELECT 3

USE $c: \backslash$ metc $\backslash$ database \data $\backslash$ inor rad.dbf

SELECT 4

USE $c: \backslash$ metc $\backslash$ database \data\develop. dbf

SELECT 5

USE $c: \backslash$ metc\database\data\technolo.dbE

SELECT 6

USE c: \metc\database\data\media.dbf

SELECT 7

USE c: \metc\database \data\pollutan.dbf

FOR $m$. index $=1$ TO 7

SELECT int (m.index)

GOTO TOP

DO WHILE.T.

IF EOF ()

EXIT

ENDIF

* define memory variable

SCATTER MEMVAR

DO CASE

* go through organic record and make all characters lower case

CASE (m.index $=1$ )

$m \cdot$ pollutant $=$ lower $(m \cdot$ pollutant $)$

m.cas num = lower (m.cas num)

$m \cdot$ polI_num = lower (m.poII_num)

go through chemical class recōrd and make all characters lower case

CASE (m.index $=2$ )

$\mathrm{m}$.group $=$ lower (m.group)

$m \cdot$ poll num $=1$ lower (m.poll num)

m.gen $\bar{c}$ lass $=$ lower $(\mathrm{m} \cdot$ gen class $)$

$\mathrm{m}$. polIutant $=$ lower $(\mathrm{m} \cdot$ polIutant $)$

m. cas num = lower (m.cas_num)

go through inorganic/rad record and make all characters lower case

CASE (m.index $=3$ )

$\mathrm{m}$. pollutant $=$ lower $(\mathrm{m} \cdot$ pollutant $)$

m.cas num = lower (m.cas num)

$\mathrm{m} \cdot$ polI num $=$ lower $(\mathrm{m} \cdot$ poIl num)

$\mathrm{m}$. daughter $=$ lower $(\mathrm{m}$. daughter $)$

m.emission 1 = lower (m.emission 1 )

m.emission $-2=1$ lower (m.emission -2 )

go through devēlop record and make $\bar{a} l 1$ characters lower case

CASE (m. index $=4$ )

$\mathrm{m}$. tech name $=$ lower $(\mathrm{m}$. tech name $)$

$m \cdot$ techñology $=$ lower $(m \cdot$ technology $)$

$m$. developer $=$ lower $(m$.developer $)$

$\mathrm{m}$. tech group = lower (m.tech_group)

$m$. domain = lower (m.domain)

m.scale $=$ lower (m.scale)

$\mathrm{m}$.rem phasel $=$ lower $(\mathrm{m}$.rem phase 1$)$

m.rem phase $2=$ lower (m.rem phase 2$)$

$\mathrm{m}$. rem phase $3=$ lower $(\mathrm{m}$.rem_phase 3$)$

m.sit $\vec{e}=$ lower (m.site)

go through technology record and make all characters lower case

CASE ( $\mathrm{m}$. index $=5$ )

m.tech group $=$ lower (m.tech group)

$\mathrm{m}$. techñology $=$ lower $(\mathrm{m}$. techñology)

m. sub class $=$ lower (m.sub class)

m.medial = lower $(m \cdot m e d i a l)$ 


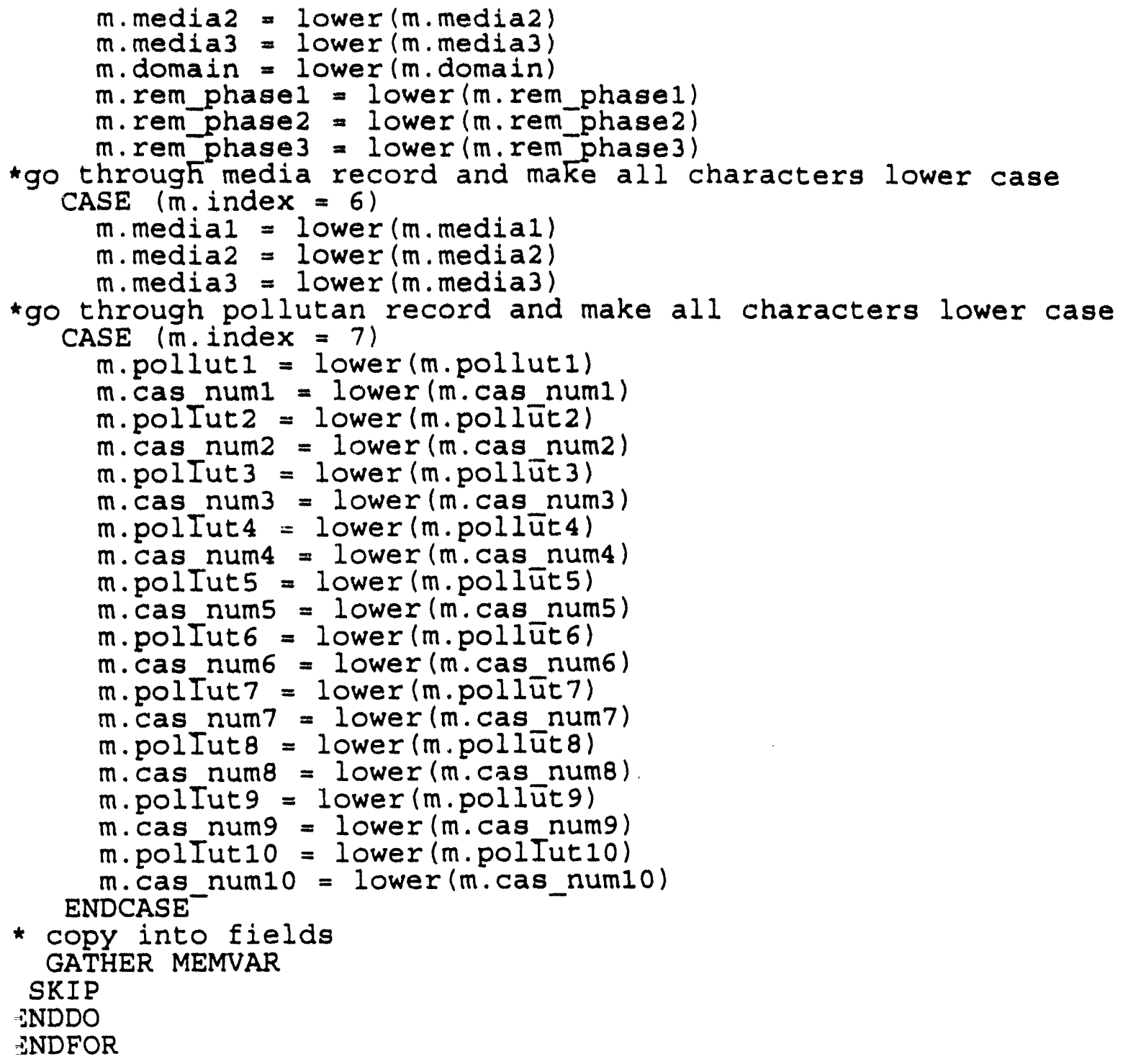


APPENDIX B

Examples From Technology Database 
Techrology:

Technology Group:

Oxidation Type:

sub Class:

Media 1:

Media 2:

Media 3 :

Domain:

Remediation Phase 1:

Remediation Phase 2 :

Remediation Phase 3: treatment

Remediation Phase 4:

Description: water

rotating biological contactor

biological

aerobic

ex-situ

\section{TECHNICAL DESCRIPTION}

The major objective of a rotating biological contactor ( $\mathrm{RBC}$ ) is the removal of soluble organic matter by its conversion to insoluble microbial cells which can be removed by sedimentation, thereby providing an effluent of high quality. Consequently, RBC's can be used as the major means of treatment for both municipal and industrial wastewaters. Like trickling filters, RBC's can also be used to achieve partial removal of soluble organic matter, and thus they find application for the pretreatment of industrial wastewaters as well.

In its simplest form, an RBC consists of parallel circular discs attached perpendicularly to a horizontal shaft which passes through their centers. The entire assembly is placed into a tank with the shaft slightly above the surface of the liquid so that the discs are approximately half immersed. Microorganisms grow on the surface of the discs and rotation of the shaft brings all of them into contact with the liquid allowing them to remove the organic matter from it. Although microbial growth results from this substrate utilization, the rotation of the discs through the liquid provides a constant shear force which causes continual sloughing of the culture, thereby maintaining a more or less constant film thickness. The rotation of the discs also mixes the liquid which keeps the stripped bionass in suspension and allows it to be carried from the reactor by the effluent. Aeration of the culture is accomplished by two mechanisms. As a point on a disc rises above the liquid surface a thin film of liquid remains attached to it and oxygen is 
transferred to that film as it is passed through the air. Rerimmersion of that point returns this highly aerobic liquid to the reactor, thereby increasing its dissolved oxygen content. In addition, a certain amount of air is entrained by the bulk of the liquid due to th eturbulence caused by the rotation of the discs. The mixing within the reactor disperses this air, thereby maintaining a relatively uniform dissolved oxygen concentration.

Although the exact composition of the microbial population on a disc depends upon the type of wastewater being treated and the relative position of the disc in the reactor, in general, the population tends to consist of more filamentous and fewer slime-forming organisms than that found on a trickling filter. Consequently, the sloughed biosolids tend to be relatively dense with good settling characteristics, although like waste activated sludge they are not particularly amenable to vacuum filtration.

As a general rule, full-scale RBC's have a disc diameter of $12 \mathrm{ft}$ and a maximum shaft length of $25 \mathrm{ft}$. Each shaft is driven by a seperate electric motot-gear reducer or by an air drive system. Although the usual rotational speed for $12 \mathrm{ft}$ diameter discs is $1.6 \mathrm{rpm}$, variable speed drives can be used for flexibility. The tankage hosing the discs is generally shallow ( $5 \mathrm{ft}$ ) and may be of either steel or concrete.

\section{REFERENCE}

Lim, Henry C., Grady, C.P. Leslie, Jr. (1980). Biological Wastewater Treatment Theory and Applications. Marcel Dekker, Inc., New York, NY. pp. $755-759$.

\section{References:}

Lim, Henry C., Grady, C.P. Leslie, Jr. (1980). Biological Wastewater Treatment Theory and Applications. Marcel Dekker, Inc., New York, NY. pp. 755-759. 
Technology:

Technology Group:

Jxidation Type:

Sub Class:

Media 1:

Media 2:

water

Media 3 : denitrification

biological

anaerobic

Domain:

ex-situ

Remediation Phase 1:

Remediation Phase 2:

Remediation phase 3: treatment

Remediation Phase 4:

pescription:

TECHNICAL DESCRIPTION

Denitrification is the reduction of nitrate nitrogen as it serves as the terminal hydrogen acceptor for microbial respiration in the absence of molecular oxygen. As such, it is an alternative to the reduction of oxygen, and thus is called anaerobic respiration. The bacteria responsible for denitrification are facultative and utilize the same biochemical pathways during both aerobic and anaerobic respiration. The only major differences are in the enzymes catalyzing the terminal electron transfer and their sites in the electron transport chain. Denitrification can be accomplished by a large number of microbial genera commonly found in wastewater treatment systems, including Achromobacter, Aerobacter, Alcaligenes, Bacillus, Flavobacterium, Micrococcus, Proteus, and Pseudomonas, thereby making the establishment of a denitrifying culture relatively easy.

There are two types of enzyme systems involved with the reduction of nitrate nitrogen: assimilatory and dissimilatory. Assimilatory nitrate reduction converts nitrate nitrogen to ammonia nitrogen for use by the cells in biosynthesis, and functions when nitrate nitrogen is the only form of nitrogen available. Dissimilatory nitrate reduction results in the formation of nitrogen gas from nitrate nitrogen, and is the one responsible for the denitrification of wastewater.

Because dissimilatory nitrate reduction serves as an alternative means of microbial respiration, there has been considerable interest in the influence of oxygen upon the responsible 
enzyme system. Denitrification probably can occur at diminished rates in the presence of oxygen, provided that anoxin conditions had previously exsisted during which enzyme synthesis could occur. One factor complicating the determination of the effects of oxygen upon denitrification in wastewater treatment systems is the flocculent nature of the cultures involved. Because of the large size of microbial floc particles there is likely to be a region in the interior which is devoid of oxygen. Thus denitrification could occur in the interior of the floc even when oxygen was present in the medium. Nevertheless, considering all of the factors known about the synthesis and activity of the enzymes responsible for denitrification, it is generally agreed that the level of dissolved oxygen should approach zero in order to achieve consistently good performance. The perspective from which denitrification must be viewed is just the opposite of that from which most wastewater treatment systems are viewed. In most systems the wastewater contains organic matter which serves as an electron donor and the designer must provide for the addition of the proper quantity of oxygen (the electron acceptor) to allow complete conversion of that organic matter to cell material and carbon dioxide. The objective during the design of a denitrification system, however, is the removal of an electron acceptor (nitrate) and to do this, a sufficient amount of electron donar (organic matter) must be available.

In the denitrification system a single-carbon compound would be an ideal candidate for the electron donar. In addition, if the electron donar is highly reduced, the TbOD per unit mass will be high, thereby minimizing the quantity which must be purchased for addition. The most highly reduced single-carbon compound is methane, but the practicality of its use is questionable. Another highly reduced single-carbon is methonal, which is widely available, of consistent quality, and relatively inexpensive. Furthermore, its yield is low, thereby minimizing the amount required. For all of these reasons, methonal is the compound usually chosenwhen an external. electron donar must be added.

Denitrification reactors fall into two broad categories: slurry reactors and Eixed Eilm reactors. The slurry reactors are quite similar to those used for activated sludge except that the mixing systems are designed to minimize oxygen transfer while maintaining the sludge in suspension. The most effective way of excluding oxygen is to used a closed reactor, but it is not uncommon to see open ones as well. There is a great deal more variety among packed tower reactors. This makes it difficult to establish a general approach to design, although one method looks promising. One aspect of the variation is in the type of media employed, which covers the range from corrugated plastic sheets to fine sand 
grains. Another is in the void spaces, which may be filied with either liuid or nitrogen gas. A third is in the fluid regime, which can range from downward in thin films to upward at a velocity sufficient to fluidize sand particles.

\section{REFERENCE}

Lim, Henry C., Grady, C.P. Leslie, Jr. (1980).

Biological Wastewater Treatment Theory and

Applications. Marcel Dekker, Inc., New York, NY. pp. $888-891$.

\section{References :}

Lim, Henry C., Grady, C.P. Leslie, Jr. (1980).

Biological Wastewater Treatment Theory and

Applications. Marcel Dekker, Inc., New York, NY. pp. $888-891$. 


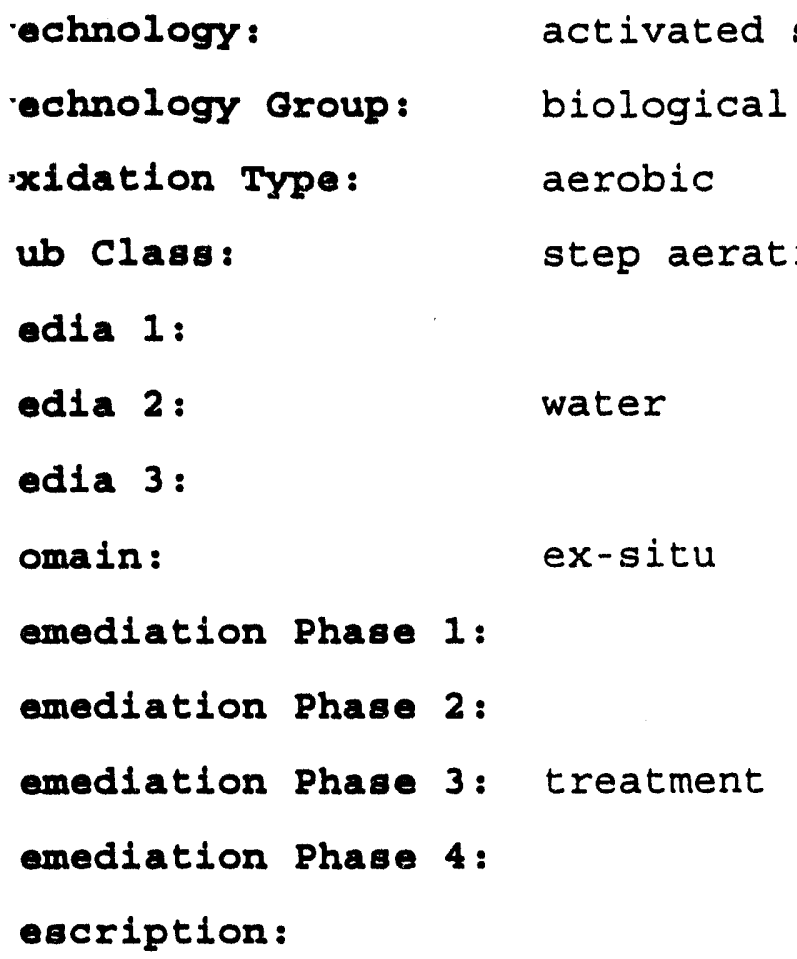

\section{TECHNICAL DESCRIPTION}

A major change in the activated sludge process came in 1942 when Gould, author of "Operating Experiences in New York City," proposed the step aeration process as a means whereby more efficient utilization could be made of the aeration capacity in plants with conventional aeration basins. The influent waste flow is split into several portions which are then fed into the aeration basin at different points, thereby giving a more even distribution of oxygen demand. Gould originally proposed a flow diagram with the initial portion of the aeration basin receiving only concentrated sludge from the final settler. The purpose of this was to regenerate the sludge prior to mixture with the incoming waste. Currently, most flow diagrams which are depicted as SAAS no longer have sludge reaeration, but instead mix the sludge and th einfluent immediately. SAAS was proposed as a way of acheiving high degrees of treatment with short space time although it utilizes MCRT's within the same range as CAS. It also provides considerable operational flexibility and consequently is still in wide use today.

\section{REFERENCE}

Lim, Henry C., Grady, C.P. Leslie, Jr. (1980).

Biological Wastewater Treatment Theory and

Applications. Marcel Dekker, Inc., New York, NY. pp. 623 . 
Lim, Henry C. 'Grady, C.P. Leslie, Jr. (1980).

Biological Wastewater Treatment Theory and

Applications. Marcel Dekker, Inc., New York, NY.

pp. 623. 
Technology:

Technology Group:

Oxidation Type:

Sub Class:

Media 1:

Media 2:

Media 3:

Domain: aerobic digestion

biological

aerobic

Remediation Phase 1:

Remediation Phase 2:

Remediation Phase 3: treatment

Remediation Phase 4:

1escription:

ex-situ

\section{TECHNICAL DESCRIPTION}

Although primary sludges may have appreciable soluble organic matter associated with them, the predominant organic matter in both primary and secondary sludges is insoluble. Consequently, the main objective of aerobic digestion is the

destruction of insoluble organic mateer in an aerobic environment--an activity which is normally performed in three types of reactors: a CSTR, a CSTR with recycle, and a batch reactor. Actually, batch reactors are seldom used in the field, their primary use being to provide design data.

Furthermore, the performance of a CSTR with recycle is the same as that of a simple CSTR as long as the MCRT of the former is equal to the space time of the latter.

Because of the relatively long MCRT's associated with aerobic digestion, a complex microbial community will normally exsist, ranging from bacteria to higher organisms such as nematodes.

There are no special physical characteristics associated with aerobic digesters although many are concrete tanks around 15 feet deep, similar in design to acivated sludge aeration chambers. Many, in fact, are converted activated sludge basins. Since the design is usually based on the assumption of complete mixing the only requirement with respect to reactor configuration is the fulfiliment of that assumption. Mixing and oxygen transfer are accomplished by both bubble and mechanical aeration using air as the oxygen source although pure oxygen has been utilized when it is used in the remainder of the plant. With the exception of pure oxygen systems the tanks are 
usually uncovered. In small plants it is common for the digesters to be operated on a fill-and-draw schedule so that the tanks are equipped with with decant pipes. In large plants, on the other hand, operation is continuous so that the settling basin is usually provided to thicken the treated sludge and separate it from the bulk of the liquid prior to discharge. Aerobic digestion is generally most applicable to secondary sludges such as those generated by the activated sludge and trickling filter processes. Because these sludges are predominantly biological solids the most important reaction will be microbial decay. Aerobic digestion has also been used on primary sludge although for best results the amount of organic material in the sludge should exceed $60 \%$. Generally, however, it has been found to be more economic to use anaerobic digestion on primary sludge even when aerobic digestion is used for the secondary sludge. This is because the large amount of nonmicrobial organic matter present will be convertd to biomass, thereby exerting a large oxygen requirement during aerobic digestion and forming much more residual sludge than would be formed by anaerobic decomposition.

\section{REFERENCE}

Lim, Henry C., Grady, C.P. Leslie, Jr. (1980). Biological Wastewater Treatment Theory and Applications. Marcel Dekker, Inc., New York, NY. pp. 690-691.

\section{References:}

Lim, Henry C., Grady, C.P. Leslie, Jr. (1980).

Biological Wastewater Treatment Theory and Applications. Marcel Dekker, Inc., New York, NY. pp. $690-691$. 
Technology:

Technology Group:

Oxidation Type:

Sub Class:

Media 1:

Media 2 :

Media 3 :

Domain: composting

biological

aerobic

windrow operation

soil

\section{Remediation Phase 1:}

Remediation Phase 2:

Remediation Phase 3: treatment

Remediation Phase 4:

Description:

GENERAL DESCRIPTION

Composting manure and sludge is a process

that involves the "microbial conversion of

biodegradable organic materials into a relatively

stable humus by thermophilic organisms under

controlled conditions." The organisms that are

used are mainly bacteria, actinomycetes, and

fungi.

Composting is usually performed under aerobic conditions, in which atmospheric oxygen is

present. "Aerobic decomposition by microorganisms

converts biodegradable organic matter in manure to

oxidized end products, primarily carbon dioxide

and water." Harmful characteristics that

correspond to the aerobic composting processes are generally constrained to odors in the beginning stages. Aerobic composting usually gives off a product with an "inoffensive odor characterized as musty and sweet."

Many factors influence biological activities and affect the rate of composting. In order for the microbial activities that cause composting to occur moisture is required. Moisture content for "aerobic thermophilic composting should be 40 to 60 percent initially." Composting materials should have voids to allow passage of air, which enhances aeration. Most likely, bulking agents are necessary to increase the porosity of fine-textured materials. Also, aeration is necessary to "(a) support aerobic microbial activity, (b) remove released moisture, and (c) remove excess heat." Adequate aeration is usually provided by one of two methods: (1) with the use of mechanical equipment, the compost pile can be turned and (2) a force-air system, in which "air is either blown or drawn into the compost." 
Furthermore, for the common types of organic wastes a $C: N: P: K$ (Carbon: Nitrogen: Phosphorus: Potassium) ratio of approximately $25: 1: 0.2: 0.8$ is desirable. Also, for the ultimate composting results the $\mathrm{pH}$ should initially be 6.5 to 7.2 . Finally, temperature is the main determinant of the rate of composting. "Thermophilic

temperatures of 130 degrees to 160 degrees

Fahrenheit are commonly achieved, providing pathogen kill and dessication of weed seeds."

\section{TECHNICAL DESCRIPTION}

The windrow process is the most typically used method of composting. In this process organic wastes are stacked into windrows and then periodically turned. "Windrows should be 3 to 5 feet tall and have a base of about 10 to 15 feet. Air movement through the porous composting material should resemble a chimney effect as internal heating occurs." In order to promote aeration mechanical equipment is used to rotate the windrow. "Windrows should be turned frequently at first and then decreased by the end of the first month. A recommended turning frequency is:

$$
\begin{aligned}
& \text { 1st week - } 3 \text { turnings } \\
& \text { 2nd week - } 1-3 \text { turnings } \\
& 3 \text { rd week - } 2 \text { turnings } \\
& 4 \text { th and 5th week - } 1 \text { turning } \\
& 5 \text { th and above - } 0 \text { turning }
\end{aligned}
$$

Temperature should be used to determine the need for turning to stimulate or control heat production."

One month of turning the windrows followed by two months in a curing pile is considered the minimum amount of composting time.

\section{Eferences:}

Sweeten, John M. "Composting Manure and Sludge." 


$\begin{array}{ll}\text { Technology: } & \text { rotary k } \\ \text { Technology Group: } & \text { thermal } \\ \text { Oxidation Type: } & \\ \text { Sub Class: } & \\ \text { Media 1: } & \text { soil } \\ \text { Media 2: } & \text { water } \\ \text { Media 3: } & \text { air } \\ \text { Domain: } & \text { ex-situ }\end{array}$

Remediation Phase 1:

Remediation Phase 2:

Remediation Phase 3: treatment

Remediation Phase 4:

Description:

GENERAL DESCRIPTION

Rotary kiln incinerators are those in which the primary chamber is a rotating cylinder lined with refractory materials. An afterburner is employed downstream from the kiln to complete the destruction of wastes. Rotary kiln incinerators operate with a wide variety of liquid, semisolid(sludge), and solid wastes, which may be burned simultaneously.

A disadvantage of the rotary kiln that may be especially troublesome with hazardous wastes is the need to maintain gastight seals at both ends of the kiln. Leakage of these seals results in fugitive emissions that can cause air pollution problems and hazards to personnel working on the unit. To minimize these problems, rotary kilns are always operated under negative pressure. The rotary kiln is considered to be the most versatile of hazardous waste incinerators.

\section{TECHNICAL DESCRIPTION}

The rotary kiln is a cylindrical, horizontei, refractory-lined shell which is mounted at a slight incline. Rotation of the shell causes mixing of the waste with the combustion air, thus improving combustion efficiency. The length to diameter ratio of the combustion chamber normally varies between $2: 1$ and $10: 1$ and the peripheral speed of rotation is normally in the range of $0.3 \mathrm{~m}$ to $1.5 \mathrm{~m}$ per minute. Combustion temperatures vary according to the characteristics of the material being incinerated but normally range from 810-1,650 degrees Celsius. Residence times vary from several seconds to hours, depending on the waste; gaseous and liquid wastes having shorter dwell times. 
Most rotary kiln installations, particularly those handling hazardous wastes, are equipped with wet scrubber emission controls. Heat recovery equipment is also common. The latter may take the form of heat exchangers to preheat combustion air or of waste heat boilers for steam generation (usually practical only in large installations).

\section{References:}

Manahan, S.E. Hazardous Waste Chemistry, Toxicology and Treatment. Lewis Publishers, Michigan. 1990

Scurlock, A. and Lindsey, A. and Fields, T. and Huber, D. Incineration in Hazardous Waste Management. United States Environmental Protection Agency. 
:echnology:

:echnology Group:

Ixidation Type:

lub clase:

ledia 1:

ledia 2 :

ledia 3 :

omain:

omediation Phase 1:

emediation Phase 2 :

emediation Phase 3: treatment

emediation Phase 4:

escription: water

ex-situ liquid injection

thermal

GENERAL DESCRIPTION

With liquid injection, freely flowing wastes

are atomized by passage through a carefully

designed nozzle. It is important that the

droplets are small enough to allow the waste to

completely vaporize and go through all the

subsequent stages of combustion while they reside

in the high-temperature zones of the incinerator.

Residence times in such incinerators are short, so nozzles especially, as well as other features must be designed for specified waste stream

characteristics such as viscosity. Certain wastes must be preheated. Nonclogging nozzles are available, but all nozzles must be carefully maintained. One of the chief costs is maintenance of refractory walls. Incinerator design is a complex, but advanced field. Many distinguishing design features are currently proprietary; especially nozzle designs and refractory composition.

Injection incinerator designs, especially nozzle design, tend to be waste specific.

However, individual designs exist for the destruction of many different liquid waste mixes: motor and industrial oils, emulsions, solvents, lacquers, and organic chemicals of all kinds including relatively hard to destroy pesticides and chemical warfare agents.

\section{TECHNICAL DESCRIPTION}

Liquid injection combustors can be used to dispose of almost all combustible liquid wastes. High viscosity, with the consequent feeding and cumbustion problems, is the important limiting factor. Liquid injection combustors are of two general types, vertical and horizontal. Normal 
temperatures maintained vary widely; from 650-1650 degrees Celsius. A typical temperature is 870 degrees Celsius. Residence times also vary, from less than $1 / 2$ second to better than one second. Normal heat release rates approximate $225,000 \mathrm{~kg}$. cal/hr-cubic meter, although for special types, heat release rates may be as high as $900,000 \mathrm{~kg}$. cal/hr. -cubic meter.

To increase the rate of vaporization and thus of combustion, the liquid wastes are atomized to present a heat transfer surface area as large as possible. Normally this is done when entering the combustor by mechanical means, by internal mixing nozzles, by two phase nozzles, by two phase nozzles, or by pressure nozzles. Droplet size is less than 40 microns in diameter. If viscosity precludes atomization, heating and mixing or other means may be necessary to reduce apparent

viscosity. A forced draft must also be supplied to the combustion chamber to provide for the necessary mixing and turbulence.

Manahan, S.E. Hazardous Waste Chemistry,

Toxicology and Treatment. Lewis Publishers, Michigan. 1990

Technologies and Management strategies for Hazardous Waste Control. United States Government, Office of Technology Assessment. Washington, D.C.

Scurlock, A. and Lindsey, A. and Fields, T. and Huber, D. Incineration in Hazardous Waste. United States Environmental Protection Agency. 1975 
Technology:

Technology Group:

\section{Oxidation Type:}

sub Class:

Media 1:

Media 2 :

Media 3:

Domain:

Remediation Phase 1:

Remediation Phase 2:

Remediation Phase 3: treatment

Remediation Phase 4:

pescription: fluidized bed combustion

thermal

soil

water

air

ex-situ

GENERAI DESCRIPTION

Fluidized-bed incinerators have a bed of granular solid(such as sand) maintained in a suspended state by injection of air. These devices can be used for Iiquid wastes or solid wastes of relatively uniform particle size. Advantages of fluidized-bed incinerators are excellent mixing, minimal requirement: for excess air, and potential to retain waste gases in the bed material.

Preheating the air injected into the bed reduces its cooling effect and enables incineration of wastes with lower heating values.

Circulating fluidized-bed combustion ( $C B C$ ) is an advanced form of fluidized-bed incinerator that has been adapted to use with mobile units on-site. This system circulates waste and sorbent solids(limestone) through a combustion chamber that is in a loop configuration.

\section{TECHNICAL DESCRIPTION}

Air driven by a blower enters a plenum at the bottom of the combustor and rises vertically through a distibutor plate into a vessel containing a bed of inert granular particles. sand is typically used as the bed material. The upward flow of of air through the sand bed results in a dense turbulent mass which behaves similarly to a liquid. Waste material to be incinerated is injected into the bed where combustion occurs within the fluidizing media. Air passage through the bed produces strong agitation of the bed particles. This promotes rapid and relatively uniform mixing of the injected waste material within the fluidized bed.

The mass of the fluidized bed is large in relation to the injected material. Bed 
temperatures are quite uniform and typically in the 1400-1600 degrees Farenheit range. At these temperatures, heat content of the fluidized bed is approximately 16,000 Btu/Cubic foot thus providing a large heat reservoir. By comparison, the heat capacity of flue gases at similar temperatures is three orders of magnitude less than a fluidized sand bed.

Heat is transferred from the bed into the injected waste materials to be incinerated. Upon reaching ignition temperature (which takes place rapidly) the material combusts and transfers heat back into the bed. Continued bed agitation by the fluidizing air allows larger waste particles to remain suspended until combustion is completed. Residual fines (ash) are carried off the bed by the exhausting flue gases at the top of the combustor. These gases are subsequently processed and/or scrubbed beffore atmospheric discharge. In specifying or designing a fluidized bed combustor, primary factors to be considered are: gas velocity; bed diameter; bed temperature; and, the type and composition of waste to be incinerated.

Manahan, S.E. Hazardous Waste Chemistry, Toxicology and Treatment. Lewis Publishers, Michigan. 1990

Sittig, M. Incineration of Indusdtrial Hazardous Wastes and Sludges. Noyes Data Corporation. New Jersey. 1979 


\section{Technology:}

Technology Group:

Oxidation Type:

\section{Sub Class:}

Media 1:

Media 2 :

Med1a 3:

Domain:

Remediation Phase 1:

Remediation Phase 2:

Remediation Phase 3: treatment

Remediation Phase 4: plasma arc

thermal soil

water

air

ex-situ

pescription:

GENERAL DESCRIPTION

Plasma incinerator systems make use of a plasma of ionized air injected through an electrical arc into which liquid wastes are injected. The extremely high 5,000-15,000 degrees Celsius temperature of the plasma breaks the waste molecules down to their constituent atoms, which remain in the elemental form or recombine to form simple molecules, such as $\mathrm{CO}$ and $\mathrm{HCl}$. The combustible gas product is burned in later stage.

TECHNICAL DESCRIPTION

The plasma torch is a device that utilizes an electrical discharge to change minute quantities of almost any gas into the hottest sustainable flame known-up to 100,000 degrees F. Plasma arc pyrolysis is possible because the "plasma Elame" can be generated in the total absence of oxygen. It is the oxygen(air) required to sustain safe combustion in conventional incinerator plants that makes pyrolysis in incinerators unattainable.

The plasma flame is generated by the following steps:

1.Gas (argon, helium, hydrogen, oxygen, etc.) is introduced in small quantity to create a swirling motion that keeps the plasma flame in the center of the torch, away from metal parts. 2. An electrical discharge is established(plasma arc flame) and maintained between the electrode inside the torch and any electrical conductor.

3. The plasma flame then converts electrical energy into heat energy in an extremely efficent manner.

In the plasma arc reactor, ther ultrahigh arc 


\begin{abstract}
temperature of 100,000 degrees $F$ is sustained to species.A plasma can be produced in several ways, the most common of which is a gaseous electrical discharge. The resultant molecular excitation leads to the formation jof a variety of metastable, atomic, free radical and ionic species.
\end{abstract}

:eferences :

Manahan, S.E. Hazardous Waste Chemistry, Toxicology and Treatment. Lewis Publishers, Michigan. 1990

Kiang, Y. and Metry, A. Hazardous Waste Processing Technology. Ann Arbor Science. 1982 
Technology:

Technology Group:

Oxidation Type:

Sub Class:

Media 1:

Media 2:

Media 3:

Domain:

Remediation Phase 1:

remediation Phase 2:

kemediation Phase 3: treatment

zemediation Phase 4:

sescription: wet air oxidation

thermal

soil

water

ex-situ

GENERAL DESCRIPTION

Wet air oxidation is the aqueous phase

oxidation of dissolved or suspended organic

subsances at elevated temperatures and pressures.

The oxygen required by wet air oxidation reactions

is provided by an oxygen containing gas, usually

air, bubbled through the liquid phase, generally

between 300 and 3000 psig. A significant advantage

of wet air oxidation is the minimal air pollution

problems that it causes. Contaminents tend to stay

in the aqueous phase. Wet air oxidation can be

used to oxidize any material, including inorganics

with a COD value. Wet air oxidation also has been

used in conjuction with biotreatment plant. In

this type of system, only $50 \%$ oxidation is

required. At this degree of oxidation, the solid

residue is approximately $90 \%$ inert. The unoxidized

organic matter is recycled to the inlet of the

treatment plant. A feature of this system is the recycling of nutrients, which can reduce of

eliminate the need to add nitrogen to the waste stream. Wet air oxidation has been used to recover chromium in treatment of sludge from a glue manufacturer. A wet air oxidation system also has been used for recovery of reusable fillers from paper mill sludges.

TECHNICAL DESCRIPTION

Organic compounds and oxidizable inorganic

species can be oxidized by oxygen in aqueous

solution. The source of oxygen usually is air.

Rather extreme conditions of temperature and

pressure are required with a temperature range of

175-327 degrees Celsius and a pressure range of

$2,070-27,700$ kilopascals. The high pressures allow

high concentration of oxygen to be dissolved in 
the water and the high temperatures enable the reaction to occur. As a first step in the initial process, the wastewater and air are brought up to pressure. An initial heating starts the reaction, after which exothermic oxidation reactions in the reaction mixture help to generate heat to keep the reaction going. the length of time that wastes are exposed to oxidation is controlled by reactor residence time. After discharge from the reactor, the treated wastewater is cooled to 35-60 degrees Celsius with incoming air/water mixture (which is preheated in the process) or with with cooling water. Gases are removed in a separator vessel. Hydrocarbon contaminant may be present in these gases. It is removed in part by wet scrubbing used to cool the gases, as well as by adsorption columns and afterburning. The oxidized liquor product may require additonal treatment, such as addition of sulfide to precipitate metals. Wet air oxidation has been applied to the destruction of cyanides in electroplating wastewaters.

\section{eferences:}

Manahan, S.E. Hazardous Waste Chemistry, Toxicology and Treatment. Lewis Publishers, Michigan. 1990 
echnology:

echnology Group:

'xidation Type:

ub Class: activated sludge

biological

aerobic

conventional activated sludge

edia 1:

edia 2 :

water

edia 3 :

omain:

ex-situ

emediation Phase 1:

emediation Phase 2:

emediation Phase 3: treatment

emediation Phase 4:

escription:

TECHNICAL DESCRIPTION

Originally the activated sludge process employed long rectangular aeration basins with the influent and recycle introduced at one end and effluent removed at the other. Consequently this is usually referred to as conventional activated sludge (CAS), although the reactor has also been called a flow-through aeration basin. However, another version makes better use of space in the plant lay-out. The usual practice with CAs was to use air diffusers spaced evenly along one wall of each basin with the same amount of air supplied to each diffuser. The rising bubbles imparted a spiral flow to the liquid thereby minimizing. longitudinal mixing. Although it was felt that this would provide plug flow, tracer studies on full-scale installations have shown the mixing pattern to be equivalent to three to five CSTR's in series. Usually CAS is designed with a MCRT between 3 and 15 days, which with typical domestic sewage gives a reactor space time (V/F) between 4 and 8 hours if the MLSS (activated sludge) concentration is around $2000 \mathrm{mg} / \mathrm{liter}$. The recycle is generally between 10 and $30 \%$ of the influent flow and sludge wastage is from either the settler underflow of the aeration basin. Because of the mixing pattern in the reactor, the reaction environment changes from point to point with the result that more oxygen is needed near the influent end where it is low. Early designs did not take this changing oxygen requirement into account with the result that portions of the basins were oxygen limited, therby hurting process efficiency. 


\section{Technology Report}

\section{REFERENCE}

Lim, Henry C., Grady, C.P. Leslie, Jr. (1980). Biological wastewater Treatment Theory and Applications. Marcel Dekker, Inc., New York, NY. pp. $621-622$.

\section{References :}

Lim, Henry C., Grady, C.P. Leslie, Jr. (1980).

Biological Wastewater Treatment Theory and

Applications. Marcel Dekker, Inc., New York, NY. pp. 621-622. 
Site Remediation Technologies: Drain-Enhanced Soil Flushing (Project MC-2A)

\author{
Quarterly Report \\ October 1 through December 31, 1993
}

\author{
M. A. Gabr \\ J. J. Bowders
}

Work Performed Under Contract No.: DE-FC21-92MC29467

\author{
For \\ U.S. Department of Energy \\ Office of Fossil Energy \\ Morgantown Energy Technology Center \\ Morgantown, West Virginia \\ By \\ West Virginia University \\ Department of Civil Engineering \\ Morgantown, West Virginia
}

February 1994 


\section{TABLE OF CONTENTS}

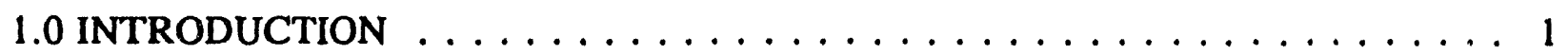

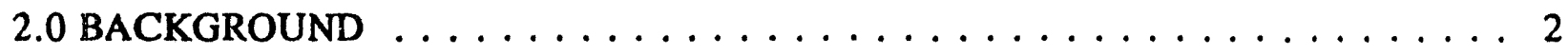

2.1 Soil Flushing as a Remediation Technique $\ldots \ldots \ldots \ldots \ldots \ldots$

2.2 PV Drains and Subsurface Liquid Movement . . . . . . . . . 3

2.3 Examples of PAHs Contaminated Sites $\ldots \ldots \ldots \ldots \ldots \ldots \ldots$

2.4Use of Surfactant to Remove Soil-Bound Organics $\ldots \ldots \ldots \ldots$

2.5 PAH Adsorption and Desorption, Degradation, and Volatilization $\ldots \ldots .7$

2.5.1 Adsorption and Desorption $\ldots \ldots \ldots \ldots \ldots \ldots \ldots$

2.5.2 Biodegradation $\ldots \ldots \ldots \ldots \ldots \ldots \ldots \ldots \ldots$

2.5.3 Volatilization $\ldots \ldots \ldots \ldots \ldots \ldots \ldots \ldots$

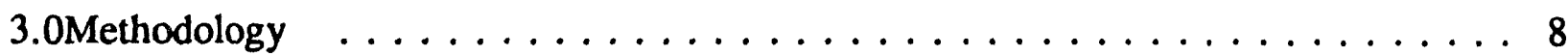

3.1 Laboratory Testing $\ldots \ldots \ldots \ldots \ldots \ldots \ldots \ldots \ldots$

3.1 .1 Batch Isotherms $\ldots \ldots \ldots \ldots \ldots \ldots \ldots \ldots$

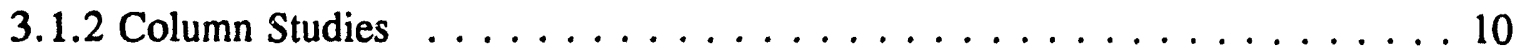

3.1.3 Procedure for Soil Extraction $\ldots \ldots \ldots \ldots \ldots \ldots \ldots \ldots \ldots$

3.1.4Contaminant Recovery Cells $\ldots \ldots \ldots \ldots \ldots \ldots \ldots$

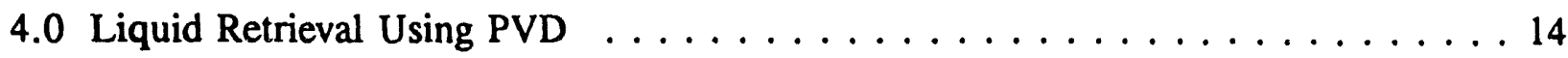

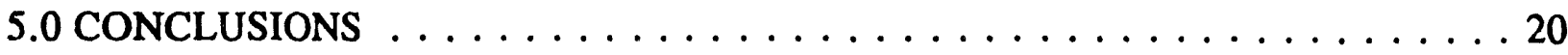




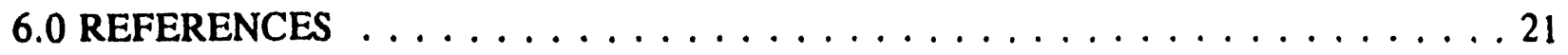

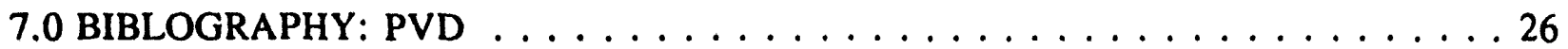

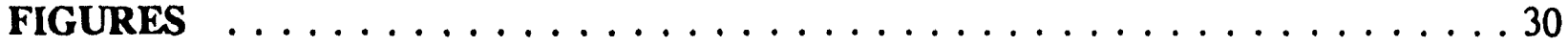




\section{LIST OF TABLES}

Table 1. Characteristics Affecting the Soil Flushing Process $\ldots \ldots \ldots \ldots$

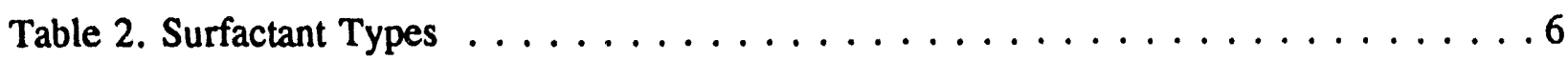

Table 3. Flexible Wall Column Tests on Study Soil $\ldots \ldots \ldots \ldots \ldots \ldots$

Table 4. Contaminant Recovery Cells and Operating Parameters . . . . . . . . . 14 


\section{LIST OF FIGURES}

Figure 1. Schematic of Conventional Soil Flushing Process $\ldots \ldots \ldots 30$

Figure 2. Field Installation Scheme of PVD System $\ldots \ldots \ldots \ldots \ldots$

Figure 3. Proposed Field Installation Scheme for Contaminant Recovery $\ldots \ldots \ldots 32$

Figure 4. Grain Size Distribution of the Study Soil $\ldots \ldots \ldots \ldots$

Figure 5. Soluability of Naphthalene Using SDS Surfactant Solution . . . . . . . . 34

Figure 6. Results of the Flexible Wall Permeability Tests: a) $w$ range of $6 \%-9 \%$ and b) $w$ range

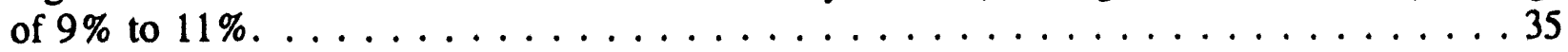

Figure 7. Dry Unit Weight Versus Molding Water Content . . . . . . . . 36

Figure 8. Variation of Permeability as a Function of Molding Water Content . . . . . . 37

Figure 9.Effect of SDS Surfactant on Measured Permeability $\ldots \ldots \ldots 38$

Figure 10. Structuer of Kaolinite Clay Used in This Study . . . . . . . . . . . . 39

Figure 11.Photograph of the Contaminant Recovery Cell $($ CRC) $\ldots \ldots \ldots 40$

Figure 12. Measured In-Place Densities from Sand Cone Tests $\ldots \ldots \ldots \ldots 1$

Figure 13. Modeling Configuration for the PVD $\ldots \ldots \ldots \ldots 4$

Figure 14. Well Pattern and Modeling Configuration $\ldots \ldots \ldots 43$

Figure 15.Relative Contaminant Concnetration as a Function of Time for Different Radial

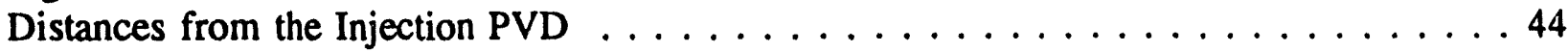




\subsection{INTRODUCTION}

Recently, the United States Environmental Protection Agency (USEPA) evaluated the progress of 112 sites where groundwater extraction was an integral part of containment and/or restoration activities (USEPA 1989a,b,c). The majority of the 112 sites were contaminated with organic compounds. The type and concentration of these organic compounds were found to be extremely diverse. In general, chemicals such as TCE, creosote, and PCBs have been reported as contaminants of concern (USEPA 1989d). In addition to the variety of contaminants and concentrations that prevail at these sites, wide variations in soil type, geological formation, and hydrogeologic conditions exist. A "typical" contaminated soil is difficult to define and remediation measures are usually developed on a site-specific basis.

There are a number of approaches to remediation that are used at contaminated sites. Examples are in-situ bioremediation, vacuum or air stripping, immobilization, and soil washing/flushing. In the case of in-situ bioremediation, organic compound(s) are biochemically degraded within the contaminated subsurface. Air or steam stripping are feasible if the Henry's Law constant of the organic compound(s) is greater than $3 \times 10^{-3} \mathrm{~atm} / \mathrm{m}^{3} \mathrm{~mol}$. In the case of immobilization techniques, contaminants are tightly bounded within a solid matrix that minimizes their migration. Solidification, stabilization, and vitrification are the principal immobilization techniques. Contrary to immobilization, the soil washing/flushing approach promotes contaminant solubilization and migration in the liquid phase so that the contaminants can be easily flushed from the soil matrix. Soil washing refers to ex-situ remediation of soils whrreas soil flushing refers to in-situ extraction of contaminants (i.e., pump and treat technology). Considering all remediation approaches, only soil flushing and stripping remove contaminant(s) from the contaminated zone without excavating the soil. Of these two, soil flushing is the process of investigation in this research proposal.

The effectiveness of the soil flushing process is limited by the extent that the contaminant is extracted from the soil and solubilized in the flushing solution, and the ease with which the flushing solution moves through the contaminated soil. Generally, for fine grained soils, both the extraction of the contaminant from the soil and movement of flushing solution are less than that observed for large grained soils (i.e., sands). Thus, soil flushing is not always effective for remediating contaminated aquifers and the use of "pump and treat systems" on extremely long term basis is often required.

The improvement of flushing solution movement in low permeability soils $\left(10^{-4}\right.$ to $\left.10^{-7} \mathrm{~cm} / \mathrm{s}\right)$ is the subject of this research proposal. Specifically, the use of Prefabricated Vertical Drains (PVD), also referred to as wick drains, to decrease the flow path and travel time between flushing solution injection and extraction points will be investigated. The development of improved flushing agents (e.g., surfactant) is not within the scope of this proposal. Rather, several flushing agents will be selected based on literature and manufacturers' information and their effectiveness in extracting contaminants from soils will be evaluated in the laboratory. The most effective extracting agent(s) will then be employed to demonstrate the feasibility of using PV drains to improve the soil flushing process. 
A creosote contaminated site has been selected as the object of remediation. The chemical composition of creosote is approximately 85 percent polycyclic aromatic hydrocarbons (PAHs), 10 percent phenolic compounds and 5 percent others (N, S, and $\mathrm{O}$ heterocyclic compounds). Thus, a single PAHs will be used as the study contaminant. Naphthalene will be used as the study contaminants.

In summary, the proposed research has as its objective the completion of the following three phases of study:

Phase 1: Quantifying the parameters that govern the release and transport of PAHs by and through the subsurface.

Phase 2: Development of a predictive mathematical model to simulate full-scale operation of PV drain-enhanced soil flushing systems.

Phase 3: Operation of a mesoscale pilot facility to investigate the performance of the PV drainenhanced soil flushing technique and to calibrate the predictive model.

Specific tasks for each phase of the research program are discussed in the Investigative Approach section of this proposal.

\subsection{BACKGROUND}

In this section background information on the conventional soil flushing system, the use of PV drains to improve liquid movement in the subsurface, and how PV drains will be used to improve the soil flushing system, will be presented. Because the focus of this research is on improving the movement and extraction of the flushing solution through the contaminated zone, the literature review will focus on liquid and mass transport in the subsurface. However, a brief discussion on PAHs contaminated sites, and the fate of PAHs in the subsurface (adsorption, desorption, biodegradation, and volatilization) is included.

\subsection{Soil Flushing as a Fenediation Technique}

In soil washing/flushing water is the primary washing fluid; chemical additives are carried by the water to promote contaminant solubilization. Non-ionic and ionic surfactant, acids, and solvents are examples of chemical additives. While soil flushing is the focus of this research, much information can be gained by examining the effectiveness of chemical additives in extracting soil-bound contaminants in the soil washing process. Soil washing refers to the exsitu remediation of soils, whereas soil flushing refers to the in-situ extraction of contaminants. Use of these two methods has been limited. However, of these two methods, there has been a greater amount of study conducted on soil washing systems, especially in Europe (USEPA, 1988a). 
The conventional soil flushing process involves a number of steps. Each step must be considered separately when assessing the overall effectiveness. The four steps are:

1) contaminant solubilization by the wash fluid (surfactant),

2) extraction of the solubilized contaminants from the subsurface,

3) treatment of the spent flushing water, and,

4) disposal/reuse of the treatment residuals.

A general schematic of the first two steps, which defines the scope of this proposed investigation, is shown in Figure 1 (Holden et al. 1989). Conditions adversely affecting the success of soil flushing process are listed in Table 1. Hydrogeologic conditions of the contaminated area are extremely important to the success of the soil flushing process. USEPA (1988b) reported that soil flushing has been used at five Superfund sites for the removal of organics. The permeability of the soils at these sites was relatively high. Currently, the soil flushing option is difficult to implement at sites with low permeabilities.

Table 1. Characteristics Affecting the Soil Flushing Process

\begin{tabular}{||l|l|}
\hline Characteristics & Reason for Potential Impact \\
\hline $\begin{array}{l}\text { Unfavorable Waste Characteristics } \\
\text { Mixed waste types } \\
\text { Variable Waste Composition }\end{array}$ & $\begin{array}{l}\text { Washing solution difficult to formulate } \\
\text { Frequent formulation of washing } \\
\text { solution }\end{array}$ \\
\hline $\begin{array}{l}\text { Unfavorable Soil Characteristics } \\
\text { Variable soil conditions } \\
\text { Low permeability }\end{array}$ & $\begin{array}{l}\text { Inconsistent flushing } \\
\text { Movement of flushing solution } \\
\text { High soil humic content } \\
\text { Soil-solvent interactions }\end{array}$ \\
$\begin{array}{l}\text { Unfavored } \\
\text { Desorption difficult } \\
\text { Difficult recovery of washing solution } \\
\text { Poor treatability of washing solution } \\
\text { Toxicity of washing solution } \\
\text { Reduction of soil permeability }\end{array}$ & $\begin{array}{l}\text { High costs } \\
\text { Replacement of washing solution } \\
\text { Health risks }\end{array}$ \\
\hline
\end{tabular}

Scurce: Technology Screening Guide for Treatment of CERCLA Soils and Sludges, U.S. EPA, 1988.

\subsection{PV Drains and Subsurface Liquid Movement}

Prefabricated vertical (PV) drains, also referred to as wick or strip drains, were originally developed as a substitute for the commonly used sand drains. They have been used extensively in the past for the expedient drainage and consolidation of low permeability soils under surface 
surcharge. The design of prefabricated vertical drains varies according to a specific application. Key parameters usually addressed in the design include:

1. Eyuivalent diameter of the drain which dictates the size of the inflow surface; and,

2. Discharge capacity of the drain .

As described by Anon (1988) PV drains consist of porous geotextiles wrapped around a plastic drainage core 3 to 4 inches wide and one quarter to $3 / 8$ of an inch thick. PV drains are usually spaced in a manner to allow the dissipation of pore pressure generated due to the application of surcharge or hydraulic stresses on the system. They provide a conduit for flow under the induced hydraulic gradient. Typically they are arranged in a triangular or square grid pattern with 3 to 12 feet spacing. Installation rates reported in the literature are on the order of 1-3 feet per second excluding equipment mobilization and set-up time. Figure 2 illustrates the installation process of a typical PV drain configuration in a field situation.

The use of synthetic PV drains has evolved to many applications and several case studies were reported in literature. A theoretical study pertaining to the design and application of vertical drainage systems was conducted by Guido (1986). Recommendations for the selection of design spacing for band-shaped PV drains were examined. A comprehensive research program was carried out by New York State Department of Transportation (Suits et al 1986) to estimate the effectiveness of several types of PV drains. This research program included laboratory and field testing. Results indicated the suitability of using PV drains to accelerate the drainage of a given soil profile.

A successful implementation of PV drains was presented by Mattox (1987). In this project, construction of a $21.7 \mathrm{ft}$ high embankment over weak marsh deposits in Mobile, Alabama, was accomplished through the use of geogrid reinforcing and PV drains. Installation of PV arains was necessary to reduce the time required for settlement of the embankment through accelerated drainage of the subsurface profile. Estimated savings due to the use of the innovative drainage technology was on the order of $\$ 600,000$. In another application, Thacker et al (1988) presented a case in which PV drains were used in the construction of a coal refuse impoundment to allow complete pore pressure dissipation. Field measurements with pneumatic piezometers indicated that the use of PV drains in the drainage scheme was successful. The coal refuse impoundment met prudent engineering criteria with regard to stability even though a large portion of the dam was being built over hydraulically filled fine coal refuse. In a similar application, Saye et al (1988) presented a case in which PV drains, installed at spacing ranging from 3 feet to 5.5 feet, were used in order to facilitate drainage and strength gain of soft highly plastic clay deposited in a cutoff oxbow of the Missouri River.

In general, research work conducted to quantify the performance of the PV drains is mostly directed toward the drainage potential to accelerate the consolidation process, or compression of a soil profile due to expulsion of water. No research specifically addressed the quantity of flow being collected using a PV drainage scheme. Furthermore, no research was conducted to 

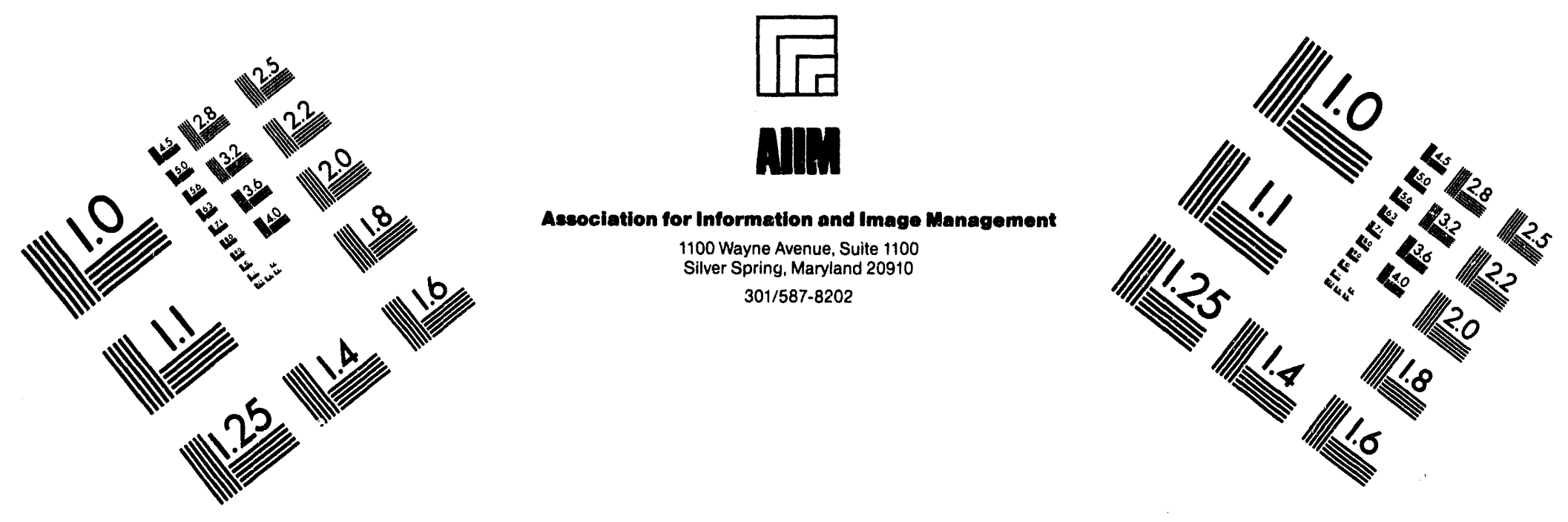

\section{Centimeter}

$\begin{array}{llllllllllllllll}1 & 2 & 3 & 4 & 5 & 6 & 7 & 8 & 9 & 10 & 11 & 12 & 13 & 14 & 15 & \mathrm{~mm}\end{array}$

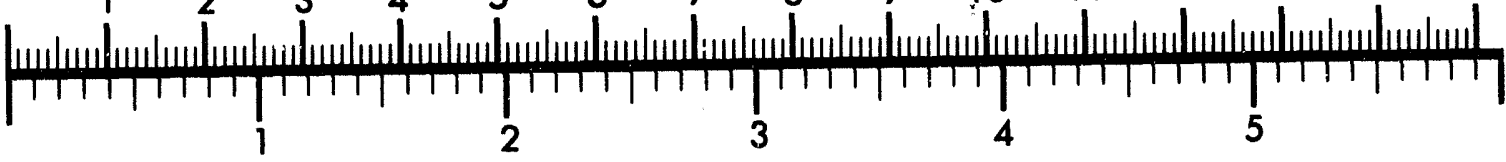
Inches
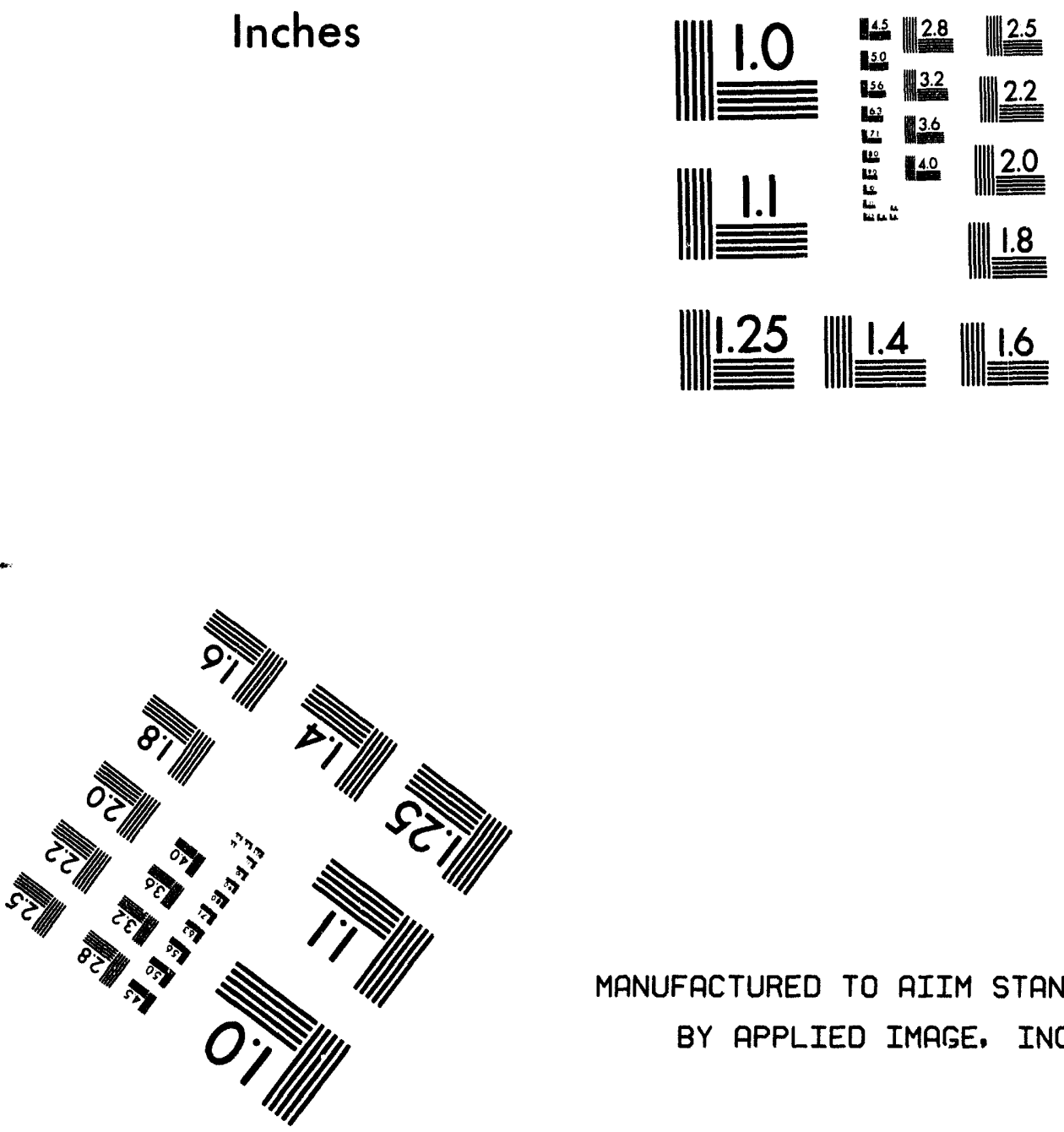

MANUFACTURED TO AIIM STANDARDS BY APPLIED IMAGE, INC.

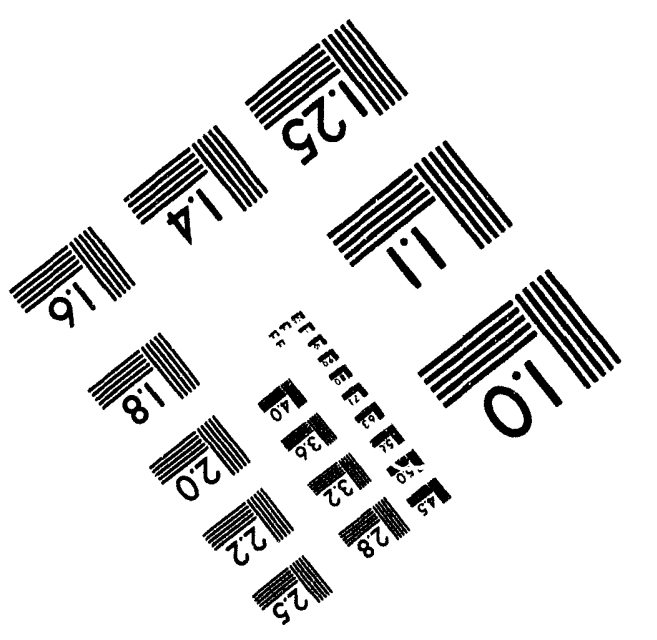



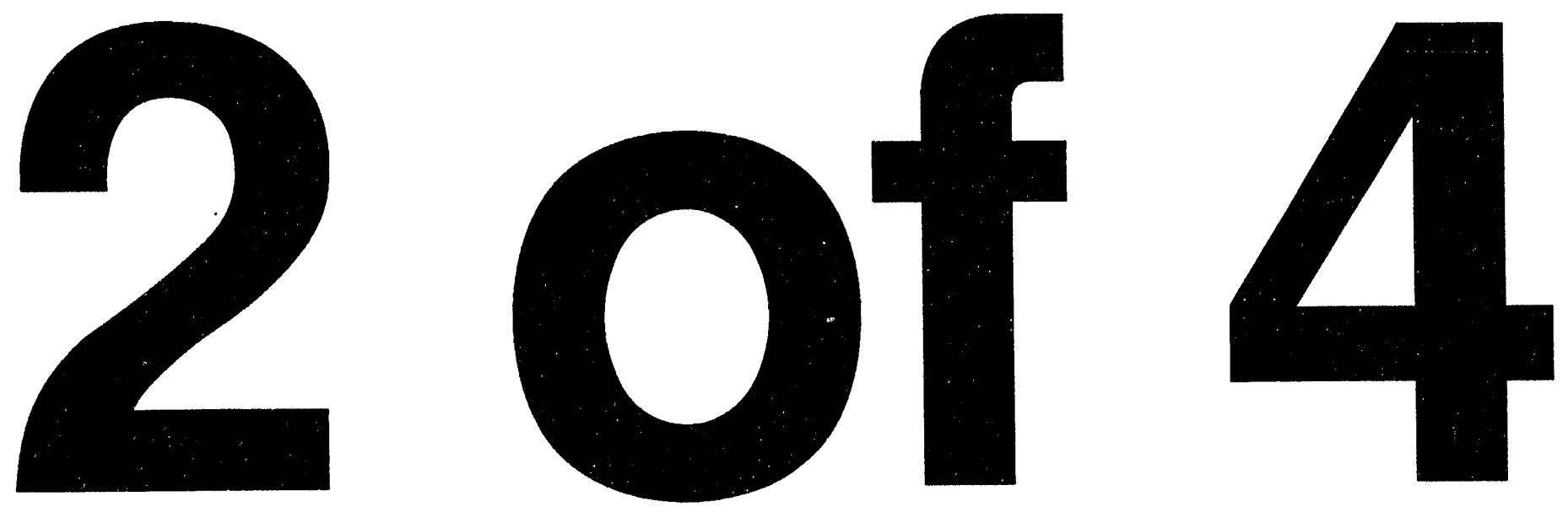
address the potential of using a PVD drainage scheme for the removal of pollutant substances from subsurface soils.

However, some research was conducted on the retrieval of tracers in agricultural applications through the use of subsurface drains. Everts and Kanwar (1990) presented the results of a study in which potassium bromide and calcium nitrate were used as tracers in sprinkler irrigation water. These tracers were applied to a field plot drained with a single subsurface drain line during two irrigations. Drain outflow was measured, and water samples were collected from drain discharge and analyzed for Nitrate $\left(\mathrm{No}^{-3}\right)$ and Bromide $(\mathrm{Br})$ content. Results indicated that, transported on a mass basis, $24 \%$ and $12 \%$ of the bromide and $20 \%$ and $9 \%$ of the nitrate reach the drain, respectively, during the two sprinkler irrigations. Although not directly related to the scope of the proposed work, the results of this study indicates the promising potential of using subsurface drains for collecting subsurface pollutants.

In summary, PV drains shorten the drainage path of the flow and therefore would expedite the soil flushing process. Compared to conventional well fields and drains, the use of PV drains would reduce both labor and material costs. Because the installation process is relatively simple and inexpensive, fluid extraction using this process can prove to be practical and cost-effective. Figure 3 schematically shows a possible arrangement for the use of PVD system for recovery of subsurface pollutants in the field.

\subsection{Examples of PAHs Contaminated Sites}

There are over 2000 wood preserving sites in the United States requiring remediation with over 50 of these sites on the National Priority List (NPL), Stinson et al. (1991). The MacGillis and Gribbs Superfund site in New Brighton, Minnesota, is heavily contaminated with creosote, pentachlorophenol, and fuel oil. PAH concentrations as high as $407 \mathrm{mg} / \mathrm{kg}$ have been reported (Stinson et al. 1991). PAH concentrations of $2,800,240$, and $10 \mathrm{mg} / \mathrm{kg}$ for peat, soils, and sediments, respectively, have been reported at the Pine Street Canal Superfund site in Burlington, Vermont, (Weir and McLane 1991). Christiansen et al. (1991) reported PAH concentration exceeding $1900 \mathrm{mg} / \mathrm{kg}$ at an undisclosed Texas site. PAH concentrations exceeding $3,000 \mathrm{mg} / \mathrm{kg}$ and $20,000 \mathrm{mg} / \mathrm{kg}$ have been reported at the Bayou Bonfouca (Slidell, Louisiana) and the Jennison-Wright (Granite City, Illinois) sites, respectively, as stated by Halloran et al. 1991. In examining contaminated sites, a wide variety of PAHs (and other organic contaminants) are present in widely ranging concentrations. Thus, remediation research must address diverse conditions. However, because the main focus of this research project is the improvement of the liquid movement phenomenon of the soil flushing process, relatively simple PAHs (e.g., naphthalene) will be chosen as the study organics. It is tacitly assumed that the PV drainenhanced soil flushing method will improve the efficiency of the pump and treat process regardless of the specific organic contaminant(s). 


\subsection{Use of Surfactant to Remove Soil-Bound Organics}

Surfactant molecules have both a polar (hydrophilic) and nonpolar (hydrophobic) segment and thus they accumulate at polar-nonpolar interfaces. Above a certain concentration surfactant will form micelles (aggregates). The polar end of the molecule in the micelles is presented to the aqueous phase. The nonpolar end of the surfactant molecule faces inward, away from water molecules. The interior of the surfactant micelles is nonpolar and thus can solubilize nonpolar compounds that are sorbed onto soil particles. A large number of commercial surfactant that are environmentally safe and relatively inexpensive are available. Surfactant types can be categorized as ionic (anionic, cationic), nonionic, and amphoteric. Examples of surfactant and their characteristics are presented in Table 2 (USEPA 1985).

Table 2. Surfactant Types

\begin{tabular}{||l|l|l|l||}
\hline Anionic & Cationic & Non-ionic & Amphoteric \\
\hline $\begin{array}{l}\text { Carboxylic acid } \\
\text { salts }\end{array}$ & Long chain amines & Alcohol ethoxylates & $\mathrm{pH}$ sensitive \\
\hline Sulfonic acid salts & $\begin{array}{l}\text { Quaternary } \\
\text { ammonium salts }\end{array}$ & $\begin{array}{l}\text { Polyoxyethylenated } \\
\text { alkylphenols }\end{array}$ & $\mathrm{pH}$ insensitive \\
\hline
\end{tabular}

The use of surfactant was first investigated by the Texas Research Instituie $(1979,1985)$ as a method of recovering gasoline. Ellis et al. (1985) investigated the use of nonionic surfactant for removal of PCBs, chlorinated phenols, and petroleum hydrocarbons from sols. At surfactant concentrations of 1.5 percent (by volume) over 90 percent of the coniaminants were removed from the soil. The use of surfactant increased removal by an order of magnitude over that observed for a water-only flush. A possible drawback to using nonionic surfactarit in the soil flushing process is the difficulty encountered in separating the surfact from the contaminated groundwater and subsequent surfactant reuse. Nash (1987) investiga id surfartant erihanced soil flushing at the lab and field-scale using soil from the Volk Air Nat wal Guar Base, Wisconsin. Significant removal of organics in the lab-scale systems wa $r \in$, no Kows in the field-scale systems was less than that observed in the rogray. The decrease in organic removal was attributed to the surfactant not penetrating the sni

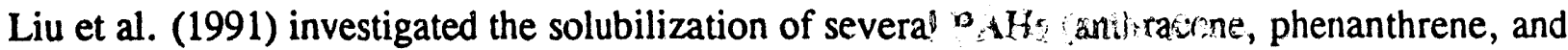
pyrene) by nonionic and anionic surfactant. Wingic surfactant octyl- and nonyl-phenylethoxylates ( 9 to 12 ethoxylate units) were ho sive. A 1 percent (by volume) surfactant dosage was required to achieve 70-90 s. subilization. Fountain and Hodge (1992) reported that several surfactant were able to inswase the solubility of common chlorinated organics (e.g., perchloroethylene (PCE)) by several order of nagnitudes. They also reported that, for PCE, several surfactant lowered interfacial tension between water and PCE to less than 1 dyne/cm causing downward vertical movement of the PCE. Thus, proper selection 
of surfactant mixtures is required if spreading of the contaminant downwards towards uncontaminated soils is to be minimized.

Dworkin et al. (1988) and Kuhn and Piotek (1989) reported favorably on the use of soil flushing for wood preserving contaminated sites. The relationship between the amount of contaminant that is solubilized and the surfactant concentration is approximately linear provided that the surfactant concentration is above the "critical" micelle concentration (CMC) as described by Gannon et al. 1989. At surfactant concentrations below the CMC contaminant solubilization does not occur. Gannon et al. (1989) reported that the CMC may be substantially reduced by the presence of a hydrophobic contaminant. However, Liu et al. (1991) reported that the solubilization of several soil-bound PAHs occurred at surfactant concentrations that were larger than the "clean-water" CMC.

In summary, a great deal of research on the development and use of surfactant to remove organic compounds from soils has been conducted. Based on results from lab-scale studies as well as full scale remediation systems, the use of surfactant in the remediation of contaminated soils appears to be promising. As mentioned previously, the focus of this research is not the development of improved surfactant but rather the improvement of the movement of the flushing solution through the contaminated zone. Thus, surfactant, such as those presented in Table 2 , will be selected based on literature and manufacturers' information. Their effectiveness in extracting contaminants from soils will be determined in the laboratory and the most effective and environmentally safe agents(s) will then be employed in the demonstration of the PV drains enhanced soil flushing process.

\subsection{PAH Adsorption and Desorption, Degradation, and Volatilization}

The fate of contaminants in the subsurface is influenced by the extent with which the contaminant adsorbs and desorbs, degrades, and volatilizes. Each of these topics will be discussed separately. The majority of the review focuses on adsorption and desorption because during the conduct of the proposed research, steps will be taken to minimize degradation and volatilization.

\subsubsection{Adsorption and Desorption}

PAHs, the study contaminant, readily accumulates on soil particles. The concentration of the soil-bound contaminant can be related to the solution concentration through a number of isotherı..s (Freundlich, Langmuir, an' B.E.T). In this study it is assumed that the soil has been contaminated thus, adsorption of the $\mathrm{PhH}$ will not be examined to a large extent. However, the adsorption of the surfactant-PAH onto clean soil will be investigated. It is conceivable that the contaminated flushing solution will pass through areas of the subsurface that are not contaminated and thus, it is possible that readsorption can occur. Desorption or solubilization of the contaminant from the soil in the presence of surfactant will be studied. 


\subsubsection{Biodegradation}

A large number of organic compounds are subject to biochemical degradation. Biological treatment of contaminated sites has been named as the remediation technique in about 15 percent of the Records of Decision (ROD) (Christiansen et al. 1991). Naphthalene, the simplest PAH, is considered relatively degradable with a BOD5/COD ratio of $<0.2$ (USEPA 1985). Christiansen et al. (1991), reported that the half-lives of total and carcinogenic PAHs in a soil-sludge slurry reactor were between 16 and 88 days. Weir and McLane (1991) reported that PAHs of low molecular weight (e.g., naphthalene and 2-methylnaphthalene) were biodegradable in soils, but higher weight PAHs were not. Berg et al. (1991) demonstrated that the composting of PAH contaminated soils was a viable treatment alternative. Total PAH soil concentration was decreased from approximately $300 \mathrm{mg} / \mathrm{kg}$ to less than $50 \mathrm{mg} / \mathrm{kg}$ in 14 weeks. Thus, it is apparent that PAHs, especially those with lower molecular weights, are susceptible to biochemical degradation. Measures to deter or account for biodegradation in laboratory experiments will be implemented.

\subsubsection{Volatilization}

PAHs are classified as semivolatile and generally have a Henry's constant lower than $10^{4}$ $\mathrm{atm} / \mathrm{m}^{3}$ mol. Organic compounds having a Henry's constant greater than $3 \times 10^{-3} \mathrm{~atm} / \mathrm{m}^{3} \mathrm{~mol}$ are candidates for air stripping (Holden et al. 1989). While volatilization of PAHs is possible, transfer of the contaminant to the gas phase is not an efficient process. However, during the conduct of this research, the loss of PAHs from the soil via volatilization will be accounted for by using a controlled experiments environment.

\subsection{METHODOLOGY}

The research program for the current phase consists of column and batch studies to determine appropriate environmental and engineering parameters for the soils, contaminants, and surfactant under consideration. In addition, several pilot scale systems (Contaminant Recovery Cells, CRC) in which the recovery efficiency (hydraulic only) of the PV drains are evaluated for uncontaminated soils.

\subsection{Laboratory Testing}

Laboratory studies are conducted to ascertain fundamental parameters of the soils, contaminant and surfactant. In addition, fundamental parameiers of the recovery system are evaluated. Laboratory work was conducted to evaluate the proper mix of the soil to be used in the testing program. A single polycyclic aromatic hydrocarbon (PAH), in this case naphthalene $\left(\mathrm{C}_{10} \mathrm{H}_{8}\right)$, is chosen as the study contanninant. Preliminary laboratory work is conducted to evaluate the proper mix of the soil to be used in the testing program. Based on the results of compaction tests according to ASTM 698D and permeability tests according to ASTM D5084 a soil blend consisting of $80 / 20$ percent by dry weight sand to kaolinite will be used. A grain size 
distribution for the study soil is shown in Figure 4.

On-going testing program includes performance of batch isotherms and flexible wall soil flushing permeability tests to estimate appropriate environmental and engineering parameters for the soils, contaminants, and surfactant under consideration. In addition, several pilot scale tests using Contaminant Recovery Cells (CRC) are underway. The recovery efficiency of the PVD system will be evaluated from these tests. Analysis to be conducted include naphthalene (by GC method 8100, USEPA, SW-846). Compaction tests according to ASTM 698D and permeability tests according to ASTM D5084 were conducted using blends of kaolinite and Ottawa sand to select the appropriate soil mix for use in the experiments.

\subsubsection{Batch Isotherms}

Batch isotherm studies are performed in order to evaluate the effectiveness of surfactant for contaminant removal from soil surfaces. A testing program was conducted to evaluate the solubilization of $\mathrm{C}_{10} \mathrm{H}_{8}$ using the stidy surfactant. Anionic aqueous solution of sodium dodecylsulfate (SDS) was chosen as the study surfactant. The empirical formula for SDS is as follows:

\section{$\mathrm{H}_{3} \mathrm{C}-\left(\mathrm{CH}_{2}\right)_{10}-\mathrm{CH}_{2} \mathrm{OSO}_{3}^{-} \mathrm{Na}^{+}$}

which has 12 carbon chains, one sulfate anion, and one sodium ion for each SDS molecule. The anionic surfactant is negatively charged which will assist in minimizing sorption to the negatively charged kaolinite particles.

Batch tests were conducted using surfactant concentrations of 0 to $0.5 \mathrm{M}$. The analysis was conducted using an HP Series II Gas Chromatograph (GC) with a capillary column and flame ionized detector. Solubilization as a function of naphthalene concentration is shown in Figure 5. As the SDS molarity increased the solubility of the naphthalene increased with a near linear relationship. This is due to the fact that the surfactant (its molecules are composed of hydrophobic and hydrophilic regions) forms micelles. which solubilize with the hydrophobic naphthalene compound. Naphthalene solubility of $500 \mathrm{mg} / \mathrm{l}$ was measured for SDS concentration of $0.05 \mathrm{Mol}$ and approximately $3300 \mathrm{mg} / \mathrm{l}$ for SDS concentration of $0.5 \mathrm{Mol}$.

Initial tests were conducted to establish the minimum size of soil samples whereby meaningful batch isotherms are obtained. Because of the nature of the test soil (80\% ottawa sand and 20\% kaolinite) and based on the results of initial tests, a minimum weight of $10 \mathrm{gm}$ is needed for representative soil sample. For samples smaller than $10 \mathrm{gm}$ the $80 \%: 20 \%$ representation was not obtained and erroneous isotherms were measured.

Adsorption of naphthalene, in the presence of the SDS surfactant, by both the sand and the kaolinite was assessed. Figure 6 shows a batch equilibrium isotherm for kaolinite mixed with approximately $50 \mathrm{mg} / 1$ of naphthalene in $0.01 \mathrm{Mol}$ SDS solution. Results from this isotherm 
indicated the potential of adsorption of the naphthalene, in $0.01 \mathrm{M}$ SDS solution, to the kaolinite. The acisorption rate was dependant on the mass of the kaolinite specimen. An average coefficient of partition (Kd) was estimated equal to 0.37 for SDS concentration of $0.01 \mathrm{M}$. Similar tests were performed on sand specimens. Virtually no adsorption was detected for the sand specimens.

A suite of tests will be performed using different concentrations of the SDS solution. These tests will be used to provide the mass of Naphthaiene adsorbed to the soil versus the concentration of the surfactant solution. Percent of contaminant removed from the soil as a function of the surfactant concentration will also be evaluated using a second suite of batch isotherm tests. Soils will be contaminated with Naphthalene by dissolving Naphthalene in Methanol, mixing the solution with soil, and allowing Methanol to evaporate. The initial soil/Naphthalene concentration will then be determined.

\subsubsection{Column Studies}

Two series of column studies were conducted. A series was conducted on clean soil with water and with surfactant solution to evaluate the hydraulic properties of the study soil. The clean soil studies were used to obtain permeability characteristics of the soil, both, with water and with surfactant as permeating liquids. The second series was conducted on contaminated soil. The tests on contaminated soil columns were used to ascertain the effectiveness of the SDS surfactant in removing contaminants under a flow type situation and estimate the dispersion/advection characteristics of the system. Sample preparation for the columns study was as follows:

1. Samples were prepared from a mixture of $80 \%$ ottawa sand and $20 \%$ kaolinite.

2. Sieve analyses were conducted as a baseline to check the final integrity of the soil upon completion of a test.

3. Samples were compacted using standard proctor according to ASTM 698D. The samples were prepared at water content range of ion will be done at a water content of approximately $6 \%$ to $11 \%$ to achieve a permeability on the order of $1 \times 10^{-5} \mathrm{~cm} / \mathrm{sec}$.

4. Flow tests are conducted to a duration necessary to provide discharge of minimum of 5-7 pore volumes of permeant fluid.

Figure 7 ( $a$ and $b$ ) shows the variation of permeability as a function of effluent pore volume. The flexible wall permeability test was conducted according to ASTM 5084 standards. The molding moisture content plays a significant role in controlling the density, and therefore the permeability, of the compacted soil. This can be explained by the diffuse dou le layer theory. At low water content, clay particles develop flocculated structures which lead to low compaction density. As the water content is increased, the structure of the soil matrix tend to disperse and high compaction densities are achieved. 
The relationship between compaction density and water content for the study soil is shown in Figure 8. Maximum density of approximately $125 \mathrm{pcf}$ was achieved in a compaction mold using Standard Proctor test and at a moisture content of $9.5 \%$. In addition, densities achieved for the samples prepared in the CRC's are presented in the figure.

The variation of the permeability as measured from the flexible wall test using ASTM 5084 standards, as a function of the molding water content is shown in Figure 9. These tests were performed using water as permeant and clean soil. As the results indicated and as reported by Lambe (1959) soils compacted dry of optimum tend to have high permeability while soils compacted wet of optimum tend to have low permeability. Measured permeabilities for the study soil decreased from $3 \times 10^{-4}$ for water content of $7 \%$ to approximately $2 \times 10^{-5}$ for molding water content of $11 \%$. Table 3 presents the soil parameters for the different column tests.

Table 3. Flexible Wall Column Tests on Study Soil

\begin{tabular}{||l|l|l|l|l|l|l|l||}
\hline Test & $\begin{array}{l}\text { Molding } \\
\text { w(\%) }\end{array}$ & $\mathrm{i}$ & $\begin{array}{l}\sigma 3 \\
(\mathrm{psi})\end{array}$ & $\begin{array}{l}\gamma \mathrm{dry} \\
(\mathrm{pcf})\end{array}$ & $\mathrm{e}$ & $\mathrm{n}$ & $\begin{array}{l}\text { Initial } \\
\text { S(\%) }\end{array}$ \\
\hline $\mathrm{k}-80 / 20-1$ & 7.5 & 30 & 5.5 & 124.6 & 0.33 & 0.25 & 61 \\
\hline $\mathrm{k}-80 / 20-2$ & 9.9 & 30 & 5.5 & 126.0 & 0.31 & 0.24 & 85 \\
\hline $\mathrm{k}-80 / 20-3$ & 11.0 & 12 & 4.0 & 121.9 & 0.36 & 0.26 & 81 \\
\hline $\mathrm{k}-80 / 20-7$ & 8.7 & 12 & 7.0 & 127.3 & 0.30 & 0.23 & 75 \\
\hline $\mathrm{k}-80 / 20-8$ & 6.8 & 12 & 7.0 & 122.2 & 0.35 & 0.26 & 48 \\
\hline $\mathrm{k}-80 / 20-9$ & 8.8 & 12 & 7.0 & 127.4 & 0.30 & 0.23 & 76 \\
\hline $\mathrm{k}-80 / 20-10$ & 9.5 & 12 & 7.0 & 126.0 & 0.31 & 0.24 & 78 \\
\hline $\mathrm{k}-80 / 20-12$ & 10.5 & 12 & 7.0 & 122.7 & 0.35 & 0.26 & 80 \\
\hline $\mathrm{k}-80 / 20-13$ & 10.3 & 12 & 7.0 & 123.7 & 0.34 & 0.25 & 80 \\
\hline
\end{tabular}

$\mathrm{i}=$ hydraulic gradient, $\sigma 3=$ confining pressure, $\mathrm{e}=$ void ratio, $\mathrm{n}=$ porosity, and $\mathrm{S}=$ degree of saturation

Figure 10 shows the effect of surfactant on the permeability characteristics of clean soil. In this case, permeability tests were performed using water as a permeant and then SDS solution with concentrations between 0.0022 and $0.008 \mathrm{Mol}$. The introduction of the SDS solution resulted in reduction of the permeability value. As shown in Figure 10, this reduction was on the order of two orders of magnitude and deserves further investigation.

The study soil contains $20 \%$ clay of the kaolinite species. The kaolinite minerals, common in soils, have a 1:1 silica: alumina 2-sheet structure with alternating silica and octahedral sheets 
as shown schematically in Figure 11. Reduction in the hydraulic conductivity may be due to one or combinations of the following factors:

1. The hydraulic conductivity depends more on the percent of clay particles in the soil mixture. A small percentage of fines can clog the pores of coarse fraction.

2. Some types of negatively charged anions such as sulfate can be attracted to the edges of the clay particles, where broken bonds create net positive charge, and becomes part of the surface. This causes deflocculations and swelling and consequently decrease in hydraulic conductivity.

3. Sulfate compounds such as SDS causes rise in the $\mathrm{pH}$ which results in solutioning of the silica tetrahedral and alumina octahedral layers. precipitation of this material can cause clogging of pores and therefore drop in the hydraulic conductivity.

Sectional grain size distribution on the soil samples was conducted to investigate potential migration of clay particles. The results indicated that no migration of the kaolinite fines took place. The composition of all tested sectional soil specimens was $80 \%$ sand and $20 \%$ kaolinite which is the original composition of the prepared samples.

The reduction in the hydraulic conductivity of the test samples due to the introduction of the surfactant was observed to be reversible. As shown in Figure 12, The hydraulic conductivity of a sample that was tested for approximately 3 months ( 50 pore volumes were circulated through it) was reduced by two orders of magnitude, as compared to values obtained using water as permeant. Then, and as the circulation of flow continued, the hydraulic conductivity values were gradually increased back to values comparable to those obtained using water as permeant. The results shown in Figure 12 are for samples contaminated with naphthalene. For the test with results obtained for 50 pore volumes, the percent removal of naphthalene $\left(\mathrm{C}_{10} \mathrm{H}_{8}\right)$ using the SDS solution was observed to be on the order of $94 \%$.

Possible explanation for this observed reduction in the hydraulic conductivity due to the introduction of the SDS solution is the solutioning of the silica and alumina and the attraction of negatively charged anions such as sulfate to the edges of the clay particles, where broken bonds create net positive charge and become part of the surface. Consequent clogging of the pores and deflocculations and swelling effect can cause the initial decrease in the hydraulic conductivity. Continuous circulation of the permeant leads to the flushing of the pores and therefore reversing the reduction phenomena.

Permeation of sand samples with SDS solution was also conducted. As shown in Figure 13, the results indicated slight to no change in the hydraulic conducivity of the sand samples.

\subsubsection{Procedure for Soil Extraction}

The extractor procedure outlined below is based on EPA Method 3540 Soxhlet Extraction. Due to financial considerations and the scope of this project, minor alterations have been made to 
accommodate the University laboratory.

Materials:

Soxhlet Extractor: $37 \mathrm{~mm} \mathrm{ID,} \mathrm{with} 250 \mathrm{ml}$ boiling flask and Allihn condenser (the cooling fluid will be the laboratory supplied cold water).

Kuderna-Danish Apparatus:

Concentrator Tube $10 \mathrm{ml}$ graduated, with ground glass stopper.

Evaporator Flask: $250 \mathrm{ml}$

Snyder Column: Three ball macro.

Boiling Chips: PTFE, 10/40 mesh.

Water Bath: Temp controlled bath under hood.

Paper Thimbles: $33 \mathrm{~mm} \times 80 \mathrm{~mm}$ cellulose.

Reagents:

Distilled Water: Laboratory supplied.

Sodium Sulfate: Granular Anhydrous.

Methylene Chloride: Pesticide grade.

Methanol: Pesticide grade.

\section{Methods:}

1. Mix $10 \mathrm{~g}$ of sample with $10 \mathrm{~g}$ of anhydrous Sodium Sulfate and place in the extractor thimble.

2. Place $175 \mathrm{ml}$ of Methylene Chloride into a $250 \mathrm{ml}$ boiling flask with boiling chips and attach to the Soxhlet extractor.

3. Attach an Allihn condenser to the extractor and install the cold water lines to the hose barbs.

4. Heat the solvent and allow to extract for $16 \mathrm{hrs}$.

5. After the extract has cooled, place the remaining solvent in to a Kuderna-Danish (K-D) concentrator.

6. Prewet the Snyder column with approx. $1 \mathrm{ml}$ of Methylene Chloride.

7. Place the concentrator in a water bath and allow the extract to evaporate until the volume left is $1 \mathrm{ml}$. The temperature should be adjusted so this takes $15 \mathrm{~min}$.

8. Remove the K-D concentrator from the bath and allow to cool. 
9. Adjust the volume if necessary with Methylene Chloride to $2 \mathrm{ml}$.

\subsubsection{Contaminant Recovery Cells}

Four contaminant recovery cells (CRC's) were constructed and are currently used to assess the efficiency of the PV drain contaminant recovery system. The CRC's include a central section $(0.67 \mathrm{~m} \mathrm{X} 0.67 \mathrm{~m} \mathrm{X}$ $1.0 \mathrm{~m}$ - length/thickness/depth of soil) which is filled with the study soil. An upstream reservoir supply a base inflow into the soil while the downstr $n$ reservoir collect the base outflow. The reservoirs are used to pre-saturate the soil and to apply a constant base flow during water and contaminant recovery tests. For contaminant recovery purposes, PV drains will be installed into the soil. In order to quantify recovery efficiency, single and multiple drains will be used. Two systems of fluid recovery are being evaluated. These include applying a vacuum to the drains and applying a surcharge on the surface of the soil. Both systems provide a driving force for fluid transport from the soil.

Figure 14 shows a photograph of one of the cells. Integrity testing was cunducted to confirm that the cells are leak-proof. Bentofix (clay/geotextile mating) was used as lining material to minimize side wall leakage. Soil samples were compacted in the CRC's and dye was introduced to trace the flow path. No side wall leakage was observed. Saturation of the soil samples in the CRC's took approximately six weeks to complete.

Figure 15 shows the distribution of the in-place density measured using the sand cone test. The uniformity of density distribution increased as the work cre: $N$ gained experience with mixing and preparing the soil samples. At this stage of the research it was decided that a density on the order of $110 \mathrm{pcf}$ is desirable in order to allow the installation of the PVD with minimum disturbance. Therefore, and as shown in Figure 15 , the molding water content was increased in preparing the sample for CRC \#3 in order to achieve the target density of $110 \mathrm{pcf}$. The permeability values measured from the CRC's as a function of time are shown in Figure 16. The permeability values measured from the CRC's show an excellent agreement with the values evaluated from the flexible wall column. The permeability values measured from the CRC's are on the order of $1 \times 10^{-4} \mathrm{~cm} / \mathrm{sec}$ and are plotted in Figure 9 for the sake of comparison with values estimated from the flexible column tests.

Feasbility study of the retrieval system favored the use of vaccum mechanis over the use of surface surcharge load for inducing the required gradient for subsurface liquid retreival. The use of surface surchage load can be achieved by using static weight (such as the use of concerete block or the use of earth fill material) or by using air bags. One disadvnatage of this concept is the possibility that the surcharge system be classified as contaminated material once it is installed on the site. A second disadvantage of the surcharge system is the length of time required to achieve a specfic stress level. In this case, a rather lengthy construction activity will br needed to install the surcharge load. This activity will be taking place in a contaminated site and extensive health and safety condieration must be observed.

Therefore, the present focus for retrieval is on the use of the vacuum system. Several preliminary pilot scale tests using Contaminant Recovery Cells (CRC) and the vacuum system were conducted. The vacuum system was successfully operated. The recovery efficiency for the mass flux was observed to be $100 \%$ using 
the PVD and the vacuum system. On-going work will characterize the efficiency of the vacuum system and the critical design parameters such as drain spacing and the magnitude of vacuum.

\subsection{Liquid Retrieval Using PVD: Model Development}

Analysis techniques for performance evaluation of the PVD focus on the ability to induce consolidation and therefore accelerate the rate of settlement at a given site with soft clay soils. However, the use of PVD for this project is to improve the soil washing process through the efficient retrieval of subsurface liquid in fine grained soils that may contain appreciable amount of cohesionless material. As shown in Figure 17, the installed PVD has a zone of influence with a radius $r_{\text {eq }}$. The governing differential equation for the solute transport assuming full saturation and no decay:

$$
\frac{\partial C}{\partial t}=\frac{1}{r} \frac{\partial}{\partial r}\left(D_{r} r \frac{\partial C}{\partial r}\right)+D_{z} \frac{\partial^{2} C}{\partial z^{2}}-\frac{1}{r} \frac{\partial}{\partial r}\left(\frac{r v_{r} C}{n}\right)-\frac{1}{n} \frac{\partial}{\partial z}\left(v_{z} C\right)-\frac{1}{n} \frac{\partial\left(\rho_{d} K_{d} C\right)}{\partial t}
$$

where:

$\mathrm{C}=$ concentration

$\mathbf{r}=$ equivalent radius

$D_{r}$ and $D_{z}=$ coefficient of vertical and radial dispersion: $f\left(v_{r}\right.$ and $\left.v_{\nu}\right)$

$v_{r}$ and $v_{z}=$ radial velocity and vertical velocity

$k_{d}=$ equilibrium distribution coefficient

$\rho_{b}=$ bulk density

$\mathrm{n}=$ porosity of soil

Using Darcy's Law, the radial velocity of flow can be defined as:

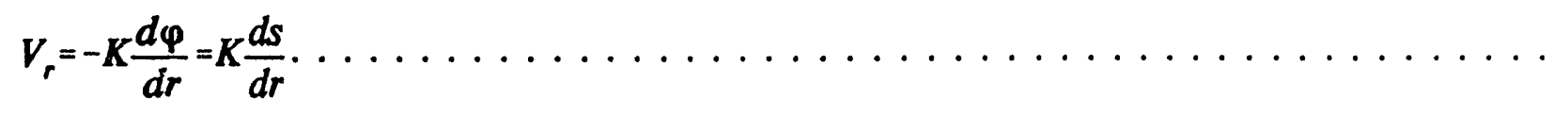

where $\mathrm{K}=$ coefficient of permeability; $\Psi=$ piezometric head, and $s=$ induced stress which in this case provide for the action of the PVDs injection/retrieval and can be expressed as follows:

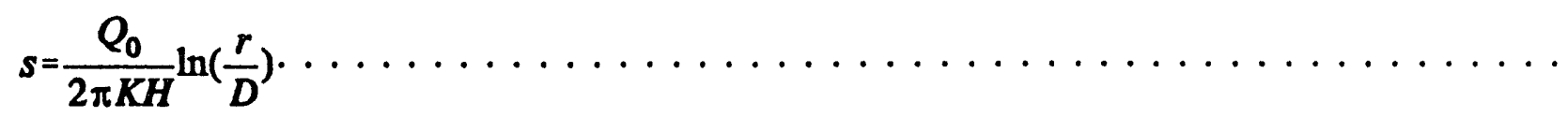

where $\mathrm{H}=$ thickness of aquifer; 
$Q_{0}=$ total discharge of injection well which is assumed equal to retrieval rate;

$\mathrm{D}=$ radial distance between injection and vacuum drains.

Substitute Eq. 3 into Eq.2, the following ewation can be obtained:

$$
y_{r}=\frac{Q_{0}}{2 \pi H r}
$$

Based on the radial velocity, the coefficient of radial dispersion is expressed as:

$$
D_{r}=\alpha_{r} v_{r}=\frac{\alpha_{r} Q_{0}}{2 \pi H r}
$$

where $\alpha_{\mathrm{r}}=$ radial dispersivity. The vertical velocity in this case can be considered as constant (Sudicky, 1982) as is expressed as follows:

$$
D_{z}=\alpha_{z} v_{z}
$$

where $\alpha_{2}=$ vertical dispersivity. Substituting Eqs.4, 5 and 6 into Eq. 1, the following model equation for axisymmetric contaminant transport can be obtained:

$$
\frac{\partial C}{\partial t}=\frac{Q_{0} \alpha_{r}}{2 \pi H r} \frac{\partial^{2} C}{\partial r^{2}}+\alpha_{2} \nu_{z} \frac{\partial^{2} C}{\partial z^{2}}-\frac{Q_{0}}{2 \pi n H r} \frac{\partial C}{\partial r}-\frac{v_{z}}{n} \frac{\partial C}{\partial z}-\frac{1}{n} \frac{\partial\left(\rho_{b} K_{d} C\right)}{\partial t}
$$

Analytical solution of above 2-order and 4-variable partial differential equation is presented as followed. Based on Carribo's solution (1952) for a similar model, C can be expressed as:

$$
C(z, r, t)=C_{z}(z, t) C_{r}(r, t)=C_{2} r_{r}
$$

where $C_{z}$ and $C_{r}$ represent vertical and radial concentration at a given location, respectively. Diffrentiating with respect to time:

$$
\begin{aligned}
& \frac{\partial C}{\partial t}=C_{z}(z, t) \frac{\partial C_{r}(r, t)}{\partial t}+C_{r}(r, t) \frac{\partial C_{z}(z, t)}{\partial t}=C_{z} \frac{\partial C_{r}}{\partial t}+C_{r} \frac{\partial C_{z}}{\partial t} \ldots \ldots \ldots \ldots \ldots \ldots \ldots \ldots \\
& \frac{\partial^{2} C}{\partial r^{2}}=C_{z} \frac{\partial^{2} C_{r}}{\partial r^{2}}
\end{aligned}
$$

Substituting Equations 9 through 13 into Eq.7, the following general equation can be obtained: 


$$
\begin{aligned}
& \frac{\partial^{2} C}{\partial z^{2}}=C_{r} \frac{\partial^{2} C_{z}}{\partial z^{2}} \cdots \\
& \frac{\partial C}{\partial r}=C_{z} \frac{\partial C_{r}}{\partial r} \cdots \\
& \frac{\partial C}{\partial z}=C_{r} \frac{\partial C_{z}}{\partial z} \cdots
\end{aligned}
$$

$$
\left(1+\frac{\rho_{b} K_{d}}{n}\right)\left(C_{r} \frac{\partial C_{z}}{\partial t}+C_{z} \frac{\partial C_{r}}{\partial t}\right)=\frac{Q_{0} \alpha_{r}}{2 \pi H r} C_{z} \frac{\partial^{2} C_{r}}{\partial r^{2}}+\alpha_{z} \nu_{z} C_{r} \frac{\partial^{2} C_{z}}{\partial z^{2}}-\frac{Q_{0} C_{z} \frac{\partial C_{r}}{\partial r}}{2 \pi n H r}-\frac{\nu_{z}}{n} C_{r} \frac{\partial C_{2}}{\partial z}
$$

$$
C_{r}\left(\frac{\partial C_{z}}{\partial t}-\frac{\alpha_{z} \nu_{z} n}{n+\rho_{b} K_{d}} \frac{\partial^{2} C_{z}}{\partial z^{2}}+\frac{\nu_{z} \frac{\partial C_{z}}{\partial z}}{n+\rho_{b} K_{d}}=C_{z}\left(\frac{-\partial C_{r}}{\partial t}+\frac{Q_{0} \alpha n \frac{\partial^{2} C_{r}}{\partial r^{2}}}{\left(n+\rho_{b} K_{d}\right) 2 \pi H r}-\frac{Q_{0} \frac{\partial C_{r}}{\partial r}}{\left(n+\rho_{b} K_{d}\right) 2 \pi H r^{\prime}}\right.\right.
$$

Equation (15) can be separated to two equations representing vertical and radial conditions as follows::

$$
\frac{\partial C_{z}}{\partial t}=\frac{\alpha_{z} v_{z} n}{n+\rho_{b} K_{d}} \frac{\partial^{2} C_{z}}{\partial z^{2}}-\frac{v_{z}}{n+\rho_{b} K_{d}} \frac{\partial C_{z}}{\partial z}
$$

with initial and boundary conditions as follows: 
$C_{z}(z, 0)=C_{x 00} \quad z z 0$

$C_{2}(0, t)=0, \quad t>0$

$C_{2}(\propto, t)=0, \quad t>0$

and

$$
\frac{\partial C_{r}}{\partial t}=\frac{\alpha_{r} Q_{0} n}{\left(n+\rho_{b} K_{d}\right) 2 \pi H} \frac{1}{r} \frac{\partial^{2} C_{r}}{\partial r^{2}}-\frac{Q_{0}}{\left(n+\rho_{b} K_{d}\right) 2 \pi H} \frac{1}{r} \frac{\partial C_{r}}{\partial r} \ldots \ldots \ldots \ldots \ldots \ldots \ldots
$$

with initial and boundary conditions as follows:

$$
\begin{aligned}
& C_{r}(r, 0)=C_{r o p} \quad r \geq r_{0} \\
& C_{r}\left(r_{0}, t\right)=0, \quad t>0 \\
& C_{r}(\propto, t)=0, \quad t>0
\end{aligned}
$$

Using Laplace Transform and Laplace Inverse Transform (Marino, 1974a), Eq.18 can be solved utilizing initial and boundary conditions:

$$
C_{z}(z, t)=\frac{C_{z 0}}{2}\left[\operatorname{erf}\left(\frac{z-\frac{v_{z} t}{n+\rho_{b} K_{d}}}{2 \sqrt{\frac{\alpha_{z} v_{z} n t}{n+\rho_{b} K_{d}}}}\right)+\operatorname{erf}\left(\frac{z+\frac{v_{z} t}{n+\rho_{b} K_{d}}}{2 \sqrt{\frac{\alpha_{z} v_{z} n t}{n+\rho_{b} K_{d}}}}\right)\right] .
$$

where

$$
\operatorname{erf}(x)=\frac{2}{\sqrt{\pi}} \int_{0}^{x} e^{-\xi^{2}} d \xi \text {. }
$$

Using Laplace Transform and Laplace Inverse Transformation as described by Marino (1974a) and utilizing initial and boundary conditions, Eq. 18 can be solved as follows:

$C_{r}(r, t)=C_{r d}[1-e r f(h)]$.

where: 


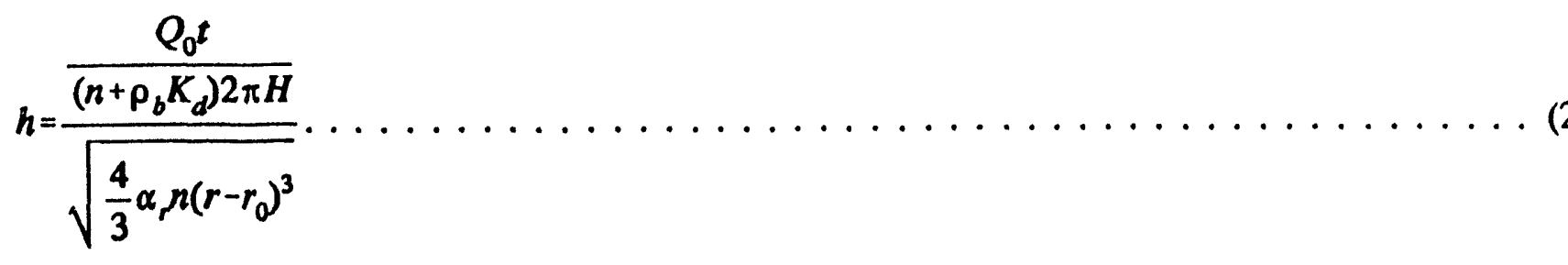

Substiryting Eq.20 and Eq.22 into Eq.8:

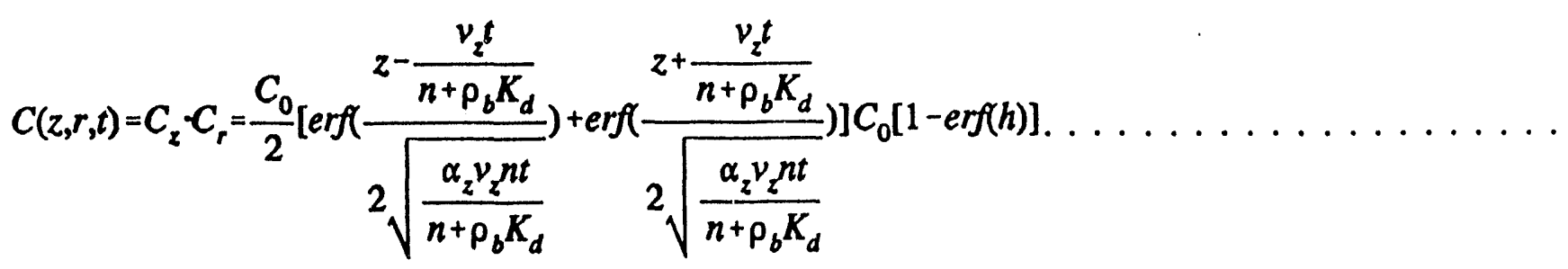

where $\mathrm{C}_{0}=\mathrm{C}_{10} \mathrm{C}_{20} ; \mathrm{h}$ and function erf are expressed by Eqs.23 and 21 . A prelinimary example is given to check the robustness of the solution. The example problem is set-up such that the relative contaminant concentration as a function of time is predicted for different radial distances from the injection well and as function of depth. The modeld configuration is shown in Figure 18. The parameters assumed in this analysis are as follows:

\begin{tabular}{|l|c|}
\hline$n$ & 0.25 \\
\hline$K_{d}$ & $1 \times 10^{3} \mathrm{~m}^{3} / \mathrm{kg}$ \\
\hline$\rho_{b}$ & $2000 \mathrm{~kg} / \mathrm{m}^{3}$ \\
\hline$H$ & $10 \mathrm{~m}$ \\
\hline$\alpha_{r}$ & $5 \mathrm{~m}$ \\
\hline$\alpha_{z}$ & $1 \mathrm{~m}$ \\
\hline$v_{z}$ & $0.5 \mathrm{~m} / \mathrm{h}$ \\
\hline$Q_{0}$ & $50 \mathrm{~m} / \mathrm{h}$ \\
\hline$C_{0}$ & $200 \mathrm{mg} / \mathrm{kg}$ \\
\hline
\end{tabular}

This example is not representative of a particular field or laboratory conditions but is rather used to illustrate the model application. It is realized that a $Q_{0}$ of $50 \mathrm{~m}^{3} / \mathrm{h}$ is rather high value. This example 
will be revised in future report. The predictions utilizing the above model is shown in Figure 19. Approximately $90 \%$ clean-up level was obtained at $3 \mathrm{~m}$ below the ground surface after one day and it took approximately $1 \mathrm{1} / 2$ days to reduce to the ori-inal concentration by $90 \%$ at depth of $7 \mathrm{~m}$ below the ground surface.

The movement of the subsurface liquid for retrieval can be induced using applied surface surcharge or injection and vacuum system. The theoretical model to estimate the maximum discharge $\left(\mathrm{q}_{\max }\right)$ to be recovered from the PVD in tay under surface surcharge is as follows:

$q_{\max }=\pi r_{e q}^{2} \frac{d U}{d e} L \epsilon$

$U=1-\exp ^{-} \frac{2 T_{R}}{f(n)}$

$F(n)=\frac{n^{2}}{\left(n^{2}-1\right)} \ln (n)-\frac{3 n^{2}-1}{4 n^{2}}$

where:

$\mathrm{U}=\quad$ average degree of consolidation

$\epsilon=\quad$ strain due to consolidation

$\mathrm{Cr}=\quad$ coefficient of radial consolidation

$\mathrm{n}=\mathrm{r}_{\mathrm{eq}} / \mathrm{r}_{\mathrm{p}}$

Modeling of the vacuum system can be achieved using the theory of wells. This effort is currently underway and is a part of the ongoing effort on model development.

\subsection{CONCLUSIONS}

In summary, the proposed work is progressing on budget and on time. Literature review was conducted 
to investigate the existing thecries dealing with applications of PVD and their applicability to the soil type to be used in the project. More laboratory tests are planned to estimate the physical and engineering properties of the test soil including Atterberg Limits, grain size distribution and compressibility characteristics. In parallel, columns and the recovery cells are being performed to characterize the mobility of the study contaminant and analyze the contaminant-soil interaction.

Permeability tests using solution of the study surfactant as permeant resulted in reduction on the order of two orders of magnitude in the values of the hydraulic conductivities and deserves further investigation. The study soil contains $20 \%$ clay of the kaolinite species. The kaolinite minerals, common in soils, have a 1:1 silica: alumina 2-sheet structure with alternating silica and octahedral sheets. Reduction in the hydraulic conductivity may be due to one or combinations of the following factors:

1. The hydraulic conductivity depends more on the percent of clay particles in the soil mixture. A small percentage of fines can clog the pores of coarse fraction.

2. Some types of negativeiy charged anions such as sulfate can be attracted to the edges of the clay particles, where broken bonds create net positive charge, and becomes part of the surface. This causes deflocculations and swelling and consequently decrease in hydraulic conductivity.

3. Sulfate ompounds such as SDS causes rise in the $\mathrm{pH}$ which results in solutioning of the silica tetrahedral and alumina octahedral layers. precipitation of this material can cause clogging of pores and therefore drop in the hydraulic conductivity.

More investigation and tests are underway to ascertain the effect of the SDS on the hydraulic behavior of the study soil. Planned tests include:

i. Sectional grain size distribution on the soil samples to investigate potential migration of clay particles.

ii. Sequential permeation with SDS solution and water to investigate whether this reduction in hydraulic conductivity is irreversible.

A contact was established with the NILEX corporation, one of the largest installers of the PVD in the U.S. The Nilex Corporation indicated strong interest in the project and discussion is underway regarding their involvement as industrial partners. In addition to contaminant recovery, results from this research program will indicate the feasibility ff using PVD system for collection and detection of leachate in cases where waste was placed in un «ned areas. Furthermore, the feasibility of using the PVD system to deploy micro-organisms for in situ bioremediation will be postulated from the results of this research.

While major strides in the hazardous waste management field have been made in minimizing the risks associated with the disposal of wastes being generated, hundreds of inactive and/or abandoned hazardous waste sites exist that require remediation. The required remediation approach varies from site to site, depending on the hazardous constituents and site conditions present. The proposed study is an initial step in what will be a continuing effort to develop an in situ method, namely PV drain-enhanced soil 
flushing, to remove PAHs and other organic compounds from the contaminated subsurface. Because there is little or no experience with using PV drains as part of the soil flushing process, the proposed study will provide fundamental and basic information on performance characteristics of the DESF process as a mean of removing contaminants from sites containing fine grained soils.

\subsection{REFERENCES}

Anon (1988) "Wick drains gain acceptance," TRNews (Transportation Research Board) No. 139 Nov-Dec 1988 pp 20-21.

Bear,J. (1967) "A general theory on hydrodynamic dispersion in porous media," I.A.S.H. Symp. On Artificial Recharge And Management Of Aquifers, Haifa, Israel, I.A.S.H., P.N.72, pp7-16.

Bear, J., (1972) Dynamics of Fluids In Porous Media, Elsevier, New York.

Bazaraa, M., and C.M Shetty (1979) "Non-linear Programming: Theory and Applications," Wiley \& Sons, New York, NY.

Berg, J.D., A.S. Eikum, T. Eggen, and H. Selfors (1991). "Cold-Climate Bioremediation: Composting and Groundwater Treatment Near the Artic Circle at a Coke Works", In Proceedings: Hazardous Materials Control/Superfund Conference, 321-325.

Cameron, D.R., and A. Klute (1977) Convective-dispersive Solute Transport with a Combined Equilibrium and Kinetic Adsorption Model. Water Resources Research, Vol. 13, 183-188.

Christiansen, J.A., C.R. Page, and J.P. Perez (1991) " Large-Scale Remediation Projects," In Proceedings: Hazardous Materials Control/Superfund Conference, pp. 127-130.

Carribo (1952) "Dispersion in stratified porous media: analytical solutions," J. of Math. and Physics, Vol.21, No.1.

Dworkin, D., D.J. Messinger, and R.M. Shapote (1988) "In-situ Flushing and Bio Reclamation Technologies at a Cresote-Based Wood Treatment Plant", In Proceedings, 5th National Conference on Hazardous Waste and Hazardous Materials, Las Vegas,Nevada.

Ellis, W.E., J.R. Pa;ne, and G.D. McNabb (1985) "Treatment of Contaminated Soils With Aqueous Surfactants," U.S. EPA Report No. EPA/600/2-85/129

Everts, C. J., and R. S. Kanwar (1990)" Estimating preferential flow to a subsurface drain with tracers," Transactions of the American Society of Agricultural Engineering, Volume 33 No. 2, Mar-Apr, pp 451-457.

Fountain J.C. and D.S. Hodge (1992). "Extraction of Organic Pollutants Using Enhanced Surfactant 
Flooding - Initial Field Test," New York State Center For Hazardous Waste Management.

Gannon, O.K., P. Bibring, K. Raney, J.A. Ward, D.J. Wilson, J.L. Underwood, and K.A. Debelak (1989) "Soil Clean Up by Ir-Situ Surfactant Flushing. III. Laboratory Results", Separation Science Technology, Vol. 24, 1073.

Guido, V. A., and N. M. Ludewig (1986)" Comparative Laboratory Evaluation of Band-Shaped Prefabricated Drains" ASTM Special Technical Publication 892, American Society for Testing Materials, Philadelphia, pp. 642-662.

Halloran, A.R., R. Troast, and D.G. Gilroy (1991) "Solvent Extraction of PAH-Contaminated Soil," In Proceedings: Hazardous Materials Control/Superfund Conference, pp. 354-361.

Holden, T. J., P. Newton, P. Sylvestri, M. Diaz, C. Baker, J.G. Herrmann, and D. Guzman (1989) "How To Select Hazardous Waste Treatment Technologies For Soils and Sludges", Noyes Data Corporation, Park Ridge, New Jersey.

Kuhn, R.C. and K.R. Piotek (1989) "A Site-Specific In Situ Treatment Process Development Program for Wood Preserving Site", R.S. Kerr Technical Assistant Program-Oily Waste, Fate, Transport, Site Characterization, and Remediation, Denver Colorado.

Liu, Z., S. Laha, and R.G. Luthy (1991) "Surfactant Solubilization of Polycyclic Aromatic Hydrocarbons in Soil-Water Suspensions," Water Science Technology Vol. 23,475-485.

Marino, M.A. (1974a) "Models of dispersion in a granular medium," J. of Hydrol., Vol23, pp313-318.

Marino, M.A. (1974b) "Numerical and analytical solutions of dispersion in a finite adsorbing porous medium," Water Res. Bull., Vol.10, 81.

Moench, A.F. and Ogata, A., (1981), "A numerical inversion of the Laplace Transform solution to radial dispersion in a porous medium," Water Resour. Res., Vol.17, No.1, pp250-252.

O'Neil, P. V., (1983), Advanced Engineering Mathematics, Wadsworth Publishing Company, Belmont, CA.

Mattox, R. M. (1987) "Geogrid Reinforcement for Cochrane Bridge Embankment" Geotextiles and Geomembranes VOL. 6, No. 1-3, pp. 225-232.

Nash. J.H. (1987) "Field Studies of I-Situ Soil Washing," U.S. EPA Report No.EPA/600/2- 87/110.

Saye, S. R., C. N. Easton, W.D. Smith, K. H. Nass, and C. C. Ladd (1988) "Experience with Wick drains in highway construction over soft clay - Storz Expressway" Transportation Research Record No. 
11591988 pp. 58-67, D.C.

Stinson, M.K., H.S. Skovronek, and W.D. Ellis (1991) "U.S. EPA SITE Demonstration of Biotrol Soil Washing Process," In Proceedings: Hazardous Materials Control/Superfund Conference, 686-690.

Suits, L. D., R. L. Gemme, and J. J. Masi (1986) "Effectiveness of Prefabricated Drains on Laboratory Consolidation of Remolded Soils" Special Technical Publication 892. American Society for Testing Materials, Philadelphia, pp 663-683.

Sun, N. Z., (1989), Mathematical Modeling of Groundwater Pollution, Geology Publishing Company, Beijing, P.R. China.

Tang, D. H. and Babu, D. K., (1979), "Analytical solution of a velocity dependent dispersion problem," Water Resour. Res., Vol.15, No.6, pp1471-1478

Thacker, B. K., C. R. Ullrich, J. G. Athanasakes, and G. Smith (1988) "Evaluation of a coal refuse impoundment built by the upstream method" Hydraulic Fill Structures, Geotechnical Special Publication No. 21. Fort Collins, CO. pp. 730-749.

Texas Research Institute (1979) "Underground Movement of Gasoline on Groundwater and Enhanced recovery by Surfactants," American Petroleum Institute.

Texas Research Institute (1985) "Test Results of Enhanced Surfactant Gasoline Recovery in Large-Scale Model Aquifer," American Petroleum Institute.

USEPA. (1985). Remedial Action at Waste Disposal Sites, U.S. Environmental Protection Agency, Office of Research and Development, Research Information Center, Cincinnati, OH EPA 625/6-85/006).

USEPA. (1988a). Assessment of International Technologies for Superfund Applications: Technology Review and Trip Report Results. U.S. Environmental Protection Agency, Office of Solid Waste and Emergency Response, Office of Emergency and Remedial Response, u40/2-88/003, Washington, D.C.

USEPA. (1988b). Technology Screening Guide for Treatment of CERCLA Soils and Sludges, U.S. Environmental Protection Agency, Office of Solid Waste and Emergency Response, Office of Emergency and Remedial Response, u40/2-88/004, Washington, D.C.

USEPA. (1989a). Evaluation of Groundwater Extraction Remedies, Volume 1 Summary Report, U.S. Environmental Protection Agency, Office of Emergency and Remedial Response, u40/2-89/054, Washington, D.C.

USEPA.(1989b). Evaluation of Groundwater Extraction Remedies, Volume 2 Case Studies 1-19, U.S. Environmental Protection Agency, Office of Emergency and Remedial Response, u40/2-89/054b, 
Washington, D.C.

USEPA. (1989c). Evaluation of Groundwater Extraction Remedies, Volume 3 General Site Data, Data Base Reports, U.S. Environmental Protection Agency, Office of Emergency and Remedial Response, u40/2-89/054c, Washington, D.C.

USEPA. (1989d). Forum on Innovative Hazardous Waste Treatment Technologies: Domestic and International, Atlanta, GA, U.S. Environmental Protection Agency, Office of Research and Development, u40/2-89/C56, Cincinnati, OH.

Van Genuchten, M. Th. (1981) " Non-Equilibrium Transport Parameters from Miscible Displacement Experiments," US Department of Agriculture, Research Report No. 119, U.S. Salinity Laboratory, Riverside, CA.

Weir, B.A. and C.L. McLane (1991) "Treatment of Coal Tar-Contaminated Soils from a Superfund Site," In Proceedings: Hazardous Materials Control/Superfund Conference, pp. 309- 314.

Wilson, D.J. (1989). Soil Clean Up by in-situ Surfactant Flushing. I. Mathematical Modeling. Separation Science and Technology, Vol. 24, No. 11, 863-892.

Wilson, D.J., and A. N. Clarke (1991) "Soil Clean Up by in-situ Surfactant Flushing. IV. A Two-Component Mathematical Model," Separation Science and Technology, Vol. 26, No. 9, 1177-1194.

Yeh, G. T., and D. S. Ward (1981) "FEMWASTE: A Finite Element Model for Waste Transport Through Saturated-Unsaturated Porous Media," ORNL-5601, Oak Ridge National Laboratory, TN. 


\subsection{BIBLOGRAPHY: PVD}

Barron, R.a. (1948) "Consolidation of Fine-Grained Soils by Drain Wells," Transactions, ASCE, vol. 113, pp. 718-747.

Carroll, R.G. (1983) "Geotextiles Filter Criteria", Transportation research Record 916, pp. 46-53.

Choa, V. (1985) "Preloading and Vertical Drains," Soil Improvement Methods, Proceedings of the Third International Geotechnical Seminar, Nanyang Technological Institute, Singapore, pp. 87-99.

Christopher, B. R. and Holtz, R.D. (1985) Geotextile Engineering Manual, FHWA, Report FHWA-TS86/203, 1044 pp.

Cortlever, N.G. (1983) "Investigation into the Behavior of Plastic Drains in Rotterdam Harbor Sludge", Geotechnics, Holland, BV, Amsterdam, 16 pp.

Fellenius, B.H. and Castonguay, N.G. (1985) "The Efficiency of Band Shaped Drains", Report, National Research Council of Canada, Department of Civil Engineering, University of Ottawa, Canada, pp. 1-54.

Furstenberg, A., Lechowicz, Z., Szymanski, A., and Wolski, W. (1983) "Effectiveness of Vertical Drains in Organic Soils," Proceedings of the Eighth European Conference in Soils Mechanics and Foundation Engineering, Helsinki, Vol. 2, pp. 611-616.

Haliburton, T.A. and Wood, P.D. (1982) "Evaluation of the U.S. Army Corps of Engineers Gradient Ratio Test for Geotextiles", Proceedings 2nd International Conference on Geotextiles, Las Vegas, Vol. I, pp. 97-101.

Hansbo, S. (1979) "Consolidation of Clay by Band-Shaped Prefabricated Drains," Ground Engineering, vol. 12, No. 5, pp. 16-25.

Hansbo, S. (1981) "Consolidation of Fine-Grained Soils by Prefabricated Drains", Proceeding of the Tenth International Conference on Soil Mechanics and Foundation Engineering, Vol. 3 pp. 677-682.

Hansbo, S. (1983a) "How to Evaluate the Properties of Prefabricated Drains", Proceedings of the Eighth European Conference on Soil Mechanics and Foundation Engineering, Helsinski, Vol. 2, pp. 621-626.

Hansbo, S. (1983b) "Techno-Economic Trend of Subsoil Improvement Methods in Foundation Engineering", Special Lectures, Proceedings of the Eighth European Conference on Soil Mechanics and Foundation Engineering, Helsinki, Vol. 3, pp. 1333-1343.

Hoare, D.J. (1982) "Synthetic Fabrics as Soil Filters: A Review", Journal of the Geotechnical 
Engineering Division, ASCE, Vol. 108, No. GT10, pp. 1240-1245.

Holtz, R. D. and Christopher, B.R. (1987) "Characteristics of Prefabricated Drains for Accelerating Consolidation," Proceedings of the Ninth European Conference on Soil Mechanics and Foundation Engineering, Dublin, (to be published).

Holtz, R.D., (1987) "Preloading with Prefabricated Vertical Strip Drains", Proceeding 1st GRI Seminar. Very Soft Soil Stabilization Using High Strength Geosynthetics, Oct 22-23, Drexel University, Phila PA. pp 104-129.

Jamiolkowski, M., Lancellotta, R., and Wolski, W. (1983) "Precompression and Speeding Up Consolidation," General Report, Specialty Session 6, Proceedings of the Eight European Conference on Soil Mechanics and Foundation Engineering, Helsinki, Vol. 3, pp. 1201-1226. Summary of Discussion, pp, 1242-1245.

Johnson, S.J. (1970) "Precompression for Improving Foundation Soils," Journal of the Soil Mechanics and Foundations Division, ASCE, Vol. 96, No. 1, pp. 145-175; also in Placement and Improvement of Soil to Support Structures, ASCE, pp. 53-86.

Kjellman, W. (1948a) "Accelerating Consolidation of Fine-Grained Soils by Means of Cardboard Wicks," Proceedings of the Second International Conference on Soil Mechanics and foundation Engineering, Rotterdam, Vol. 2, Subsection 9d1, pp. 302-305.

Kjellman, W. (1948b) Discussion to "Consolidation of Fine-Grained Soils by Drain Wells,' Transactions, ASCE, Vol. 113, pp. 748-751.

Koerner, R.M. and Bove, J.A. (1983) "In Plane Hydraulic Properties of Geotextiles", Geotechnical Testing Journal, ASTM, Vol. 6, No. 4, pp. 190-195.

Koerner, R.M. (1990) Designing with Geosynthetics, Prentice-Hall, Inc., Englewood Cliffs, NJ, 652 pp. 2nd Ed.

Koerner R.M., Fowler, J., and Lawrence, C.A. (1986) "Soft Soil Stabilization Study for Wilmington Harbor South Dredge Disposal Area", Miscellaneous Paper GL-86-38, USAE Waterways Experiment Station, Vicksburg, MS. 81 pp.

Koerner, R.M. (1984) Construction and Geotechnical Methods in Foundation Engineering, McGrawHill, New York, Chapter 5.3 p 246-256.

Holtz, R.D., (1987) "Preloading with Prefabricated Vertical Strip Drains", Proceeding 1st GRI Seminar, Very Soft Soil Stabilization Using High Strength Geosynthetics, Oct 22-23, Drexel University, Phila PA. pp 104-129.

Kremer, R. (1983) "Discussion to Specialty Session … 6, Proceedings of the Eighth European 
Conference on Soil Mechanics and Foundation Engineering, Helsinki, Vol. 3, pp. 1235-1237.

Kremer, R.H.J., Oostveen, J.P., Van Weele, A.F., De Jager, W.F.J. and Meyvogel, I.J. (1983) "The Quality of Vertical Drainage", Proceedings of the Eighth European Conference on Soil Mechanics and Foundation Engineering, Helsinki, Vol. 2, pp. 721-726.

Lawrence, C.A. and Koerner, R.M. (1988) "Flow Behavior of Kinked Strip Drains", Symposium on Geosynthetics for Soil Improvement, ASCE Spring Convention, Nashville pp. 22-39.

Lawson, C. R. (1982) "Filter Criteria for Geotextiles: Relevance and Use", Journal of the Geotechnical Engineering Division, ASCE, Vol. 108, No. GT10, pp. 1300-1317.

Mitchell, J.K. (1981) "Soil Improvement--State-of-the-Art Report,' Session 12, Proceedings of the Tenth International Conference on Soil Mechanics and Foundation Engineering, Stockholm, Vol. 4, pp. 506-565.

Oostveen, J.P. (1984) "Inspectie van Opgegraven Verticale Drains in Het veld met Aanvullend Laboratoriumonderzoek" Rapport No. 235, Labaoratorium voor Geotechniek, Technical University of Delft, 26. pp.

Rankilor, P.R. (1981) Membranes in Ground Engineering, John Wiley and Sons.

Rixner, J.J., Kraemer, S.R., and Smith, A.D. (1986) "Prefabricated Vertical Drains, Vol. 1: Engineering Guidelines," Federal Highway Administration, Report No. FHWA-RD-86/168, 117 p.

Schober, W. and Teindl, H. (1979) "Filter Criteria for Geotextiles", Proceedings of the 7th European Conference on Soil Mechanics and Foundation Engineering, Brighton, Vol. 2, pp. 121-129.

Suits, D.L. (1986) "New York State Department of Transportation's (NYDOT) Basis for Acceptance and Specification for Prefabricated Wick Drains", Transportation Research Circular 309, pp. 7-9.

Suits, L.D., Gemme, R.L. and Masi, J.J. (1986) "Effectiveness of Prefabricated Drains on Laboratory Consolidation of Remolded Soils", Consolidation of Soils: Testing and Evaluation, ASTM STP 892, pp. 663-683.

TRB (1986) "Shared Experience in Geotechnical Engineering: Wick Drains, " Transportation Research Circular, No. 309, Transportation Research Board, 15 pp.

van de Griend, A.A. (1984) "Vervorming van Kunststof Drains", Veg en Water, No. 1, (Oct.), 7 pp. Veldhuijzen van Zanten, R. (1986) "The Guarantee of the Quality of Vertical Drainage Systems", 
Proceedings of the Third International Conference on Geotextiles, Vienna, Vol. 3, pp. 651-655.

Warren, M.J. (1986) "Impacts of Design and Specification Requirements on Actual Wick Drain Construction", Transportation Research Circular 309, pp. 10-13. 


\section{FIGURES}




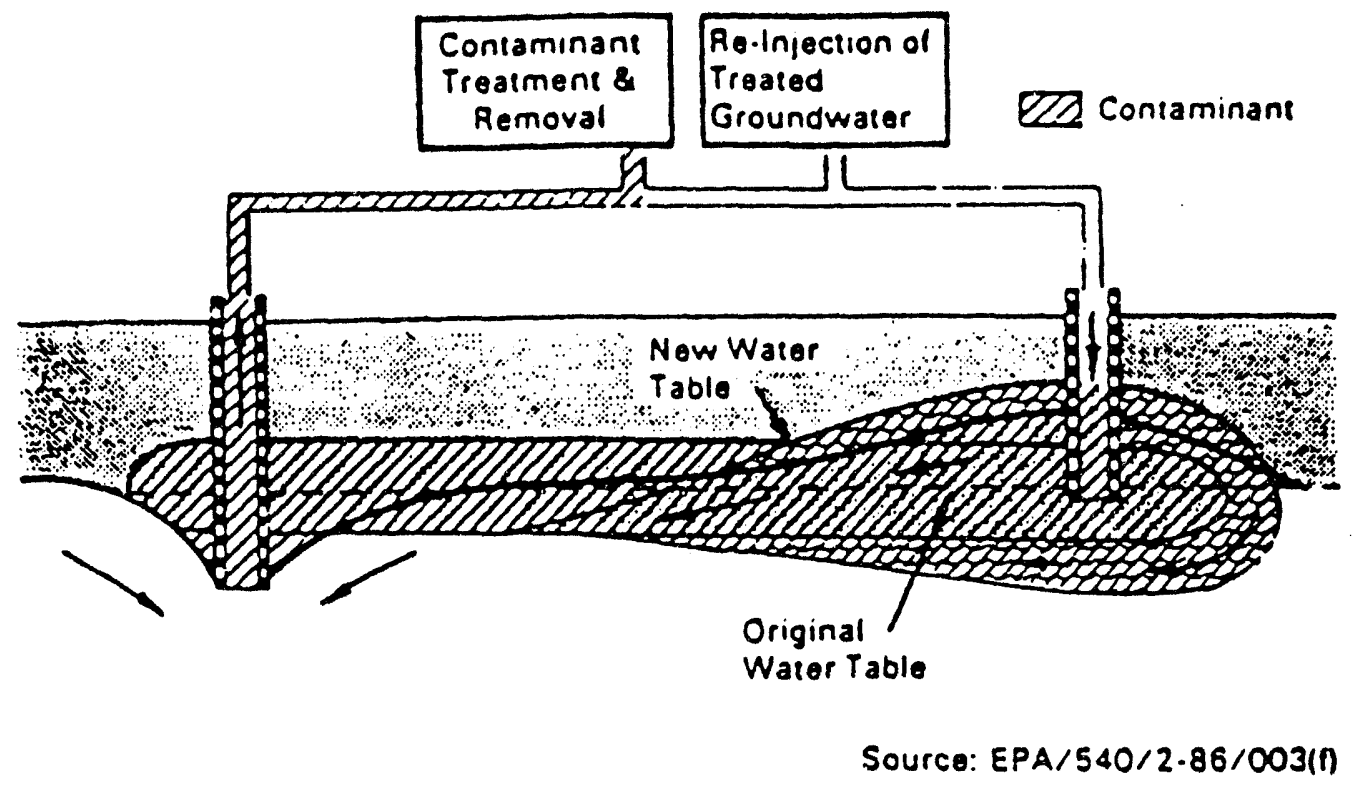

Figure 1. Schematic of Conventional Soil Flushing Process 


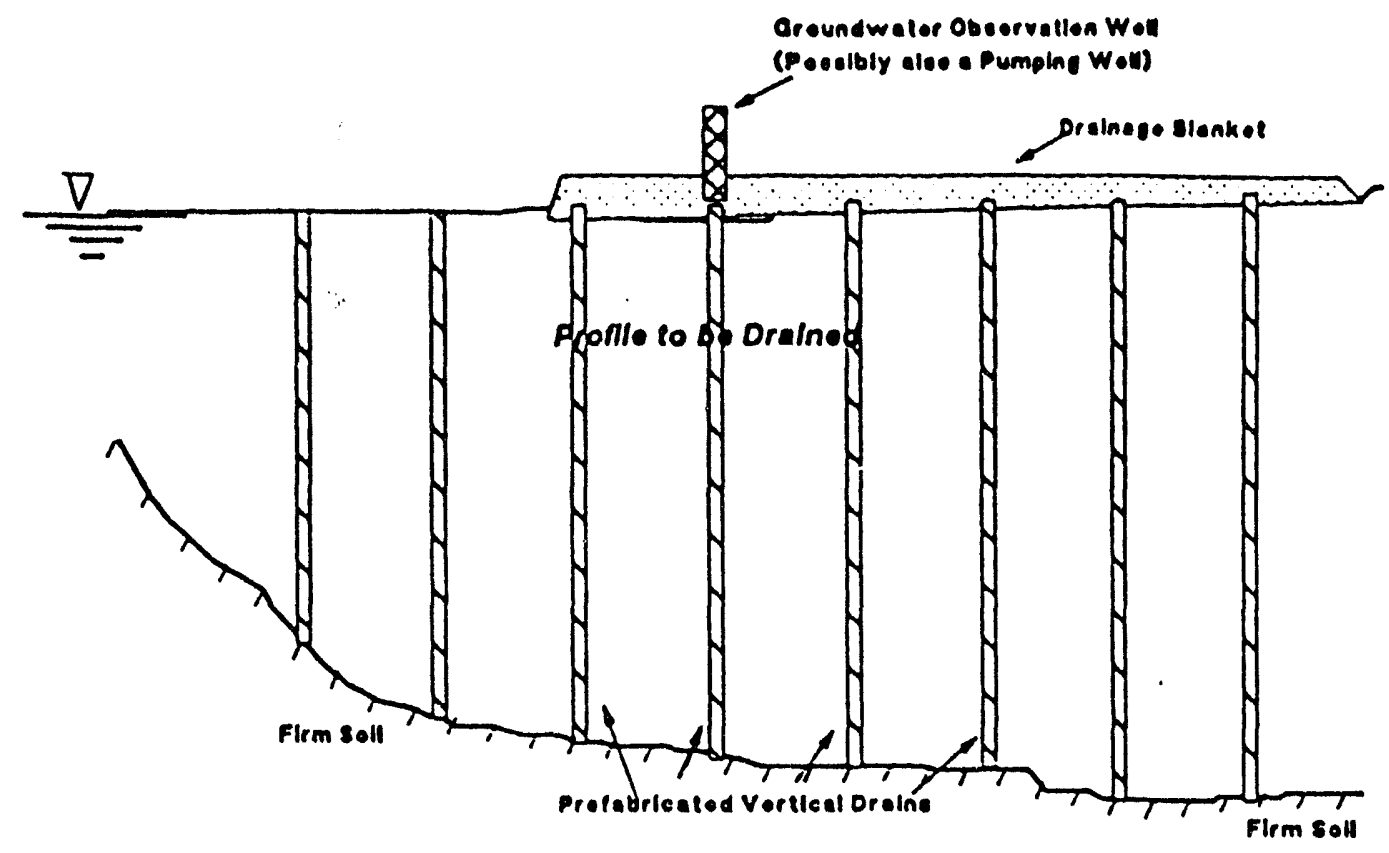

Figure 2. Flold Installation Scheme of PVD System

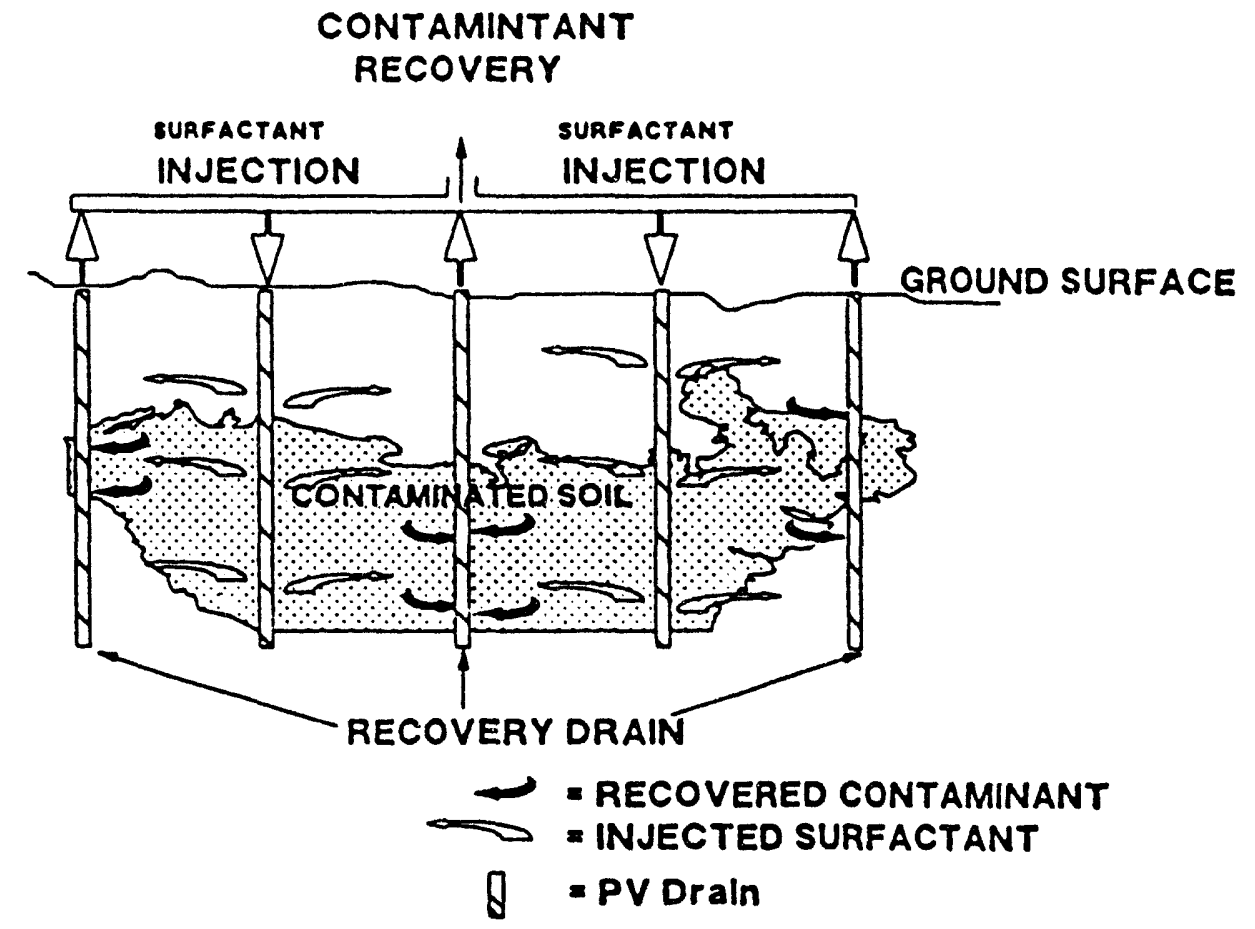

Figure 3. Proposed Fleld Installation Scheme for Contaminant Recovery 


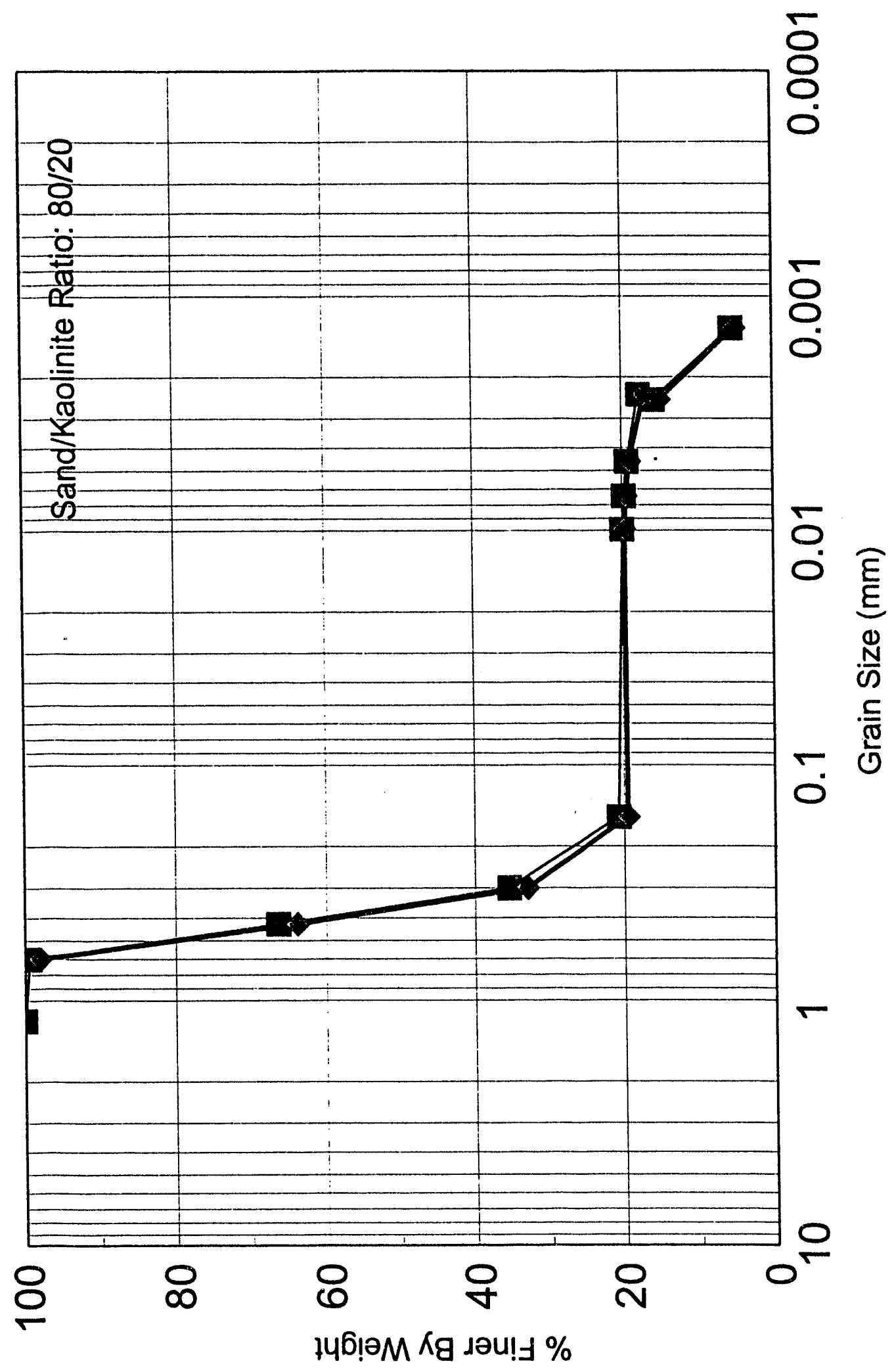

Figure 4. Grain Size Distribution of the Study Soil 


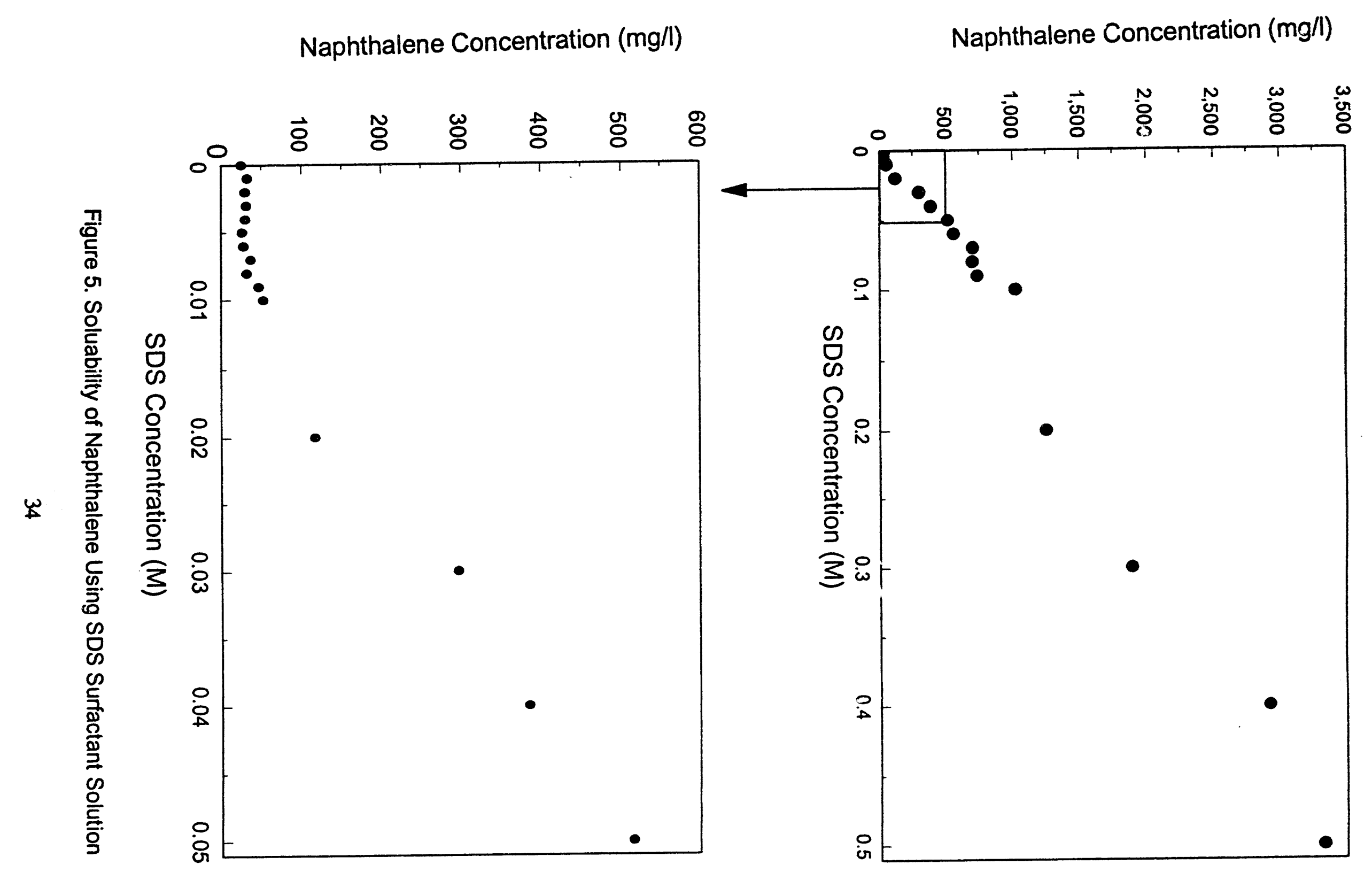




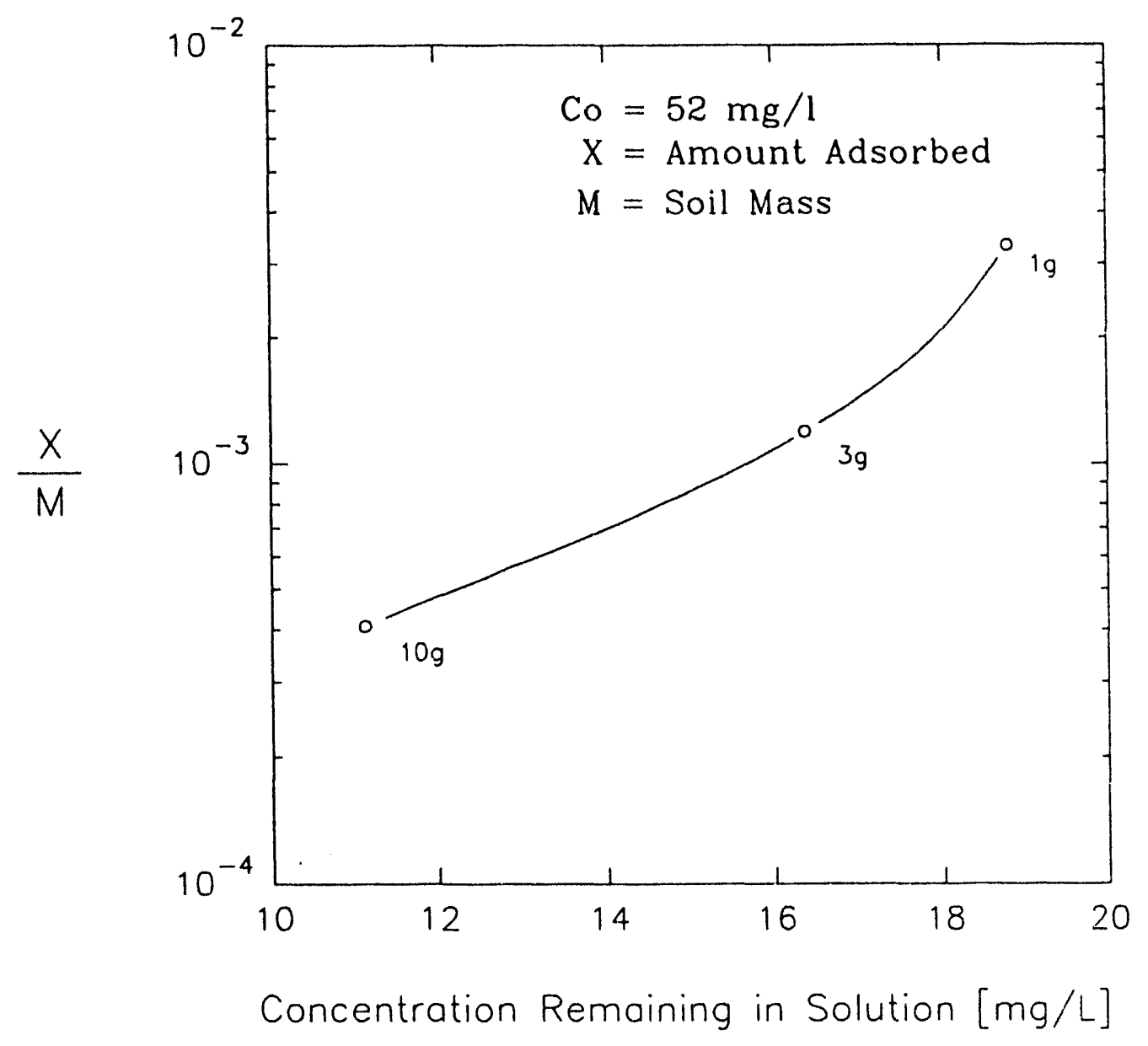

Figure 6. Batch Isotherm for Kaolinite-Naphthalene in 0.01 Mol SDS Solution 


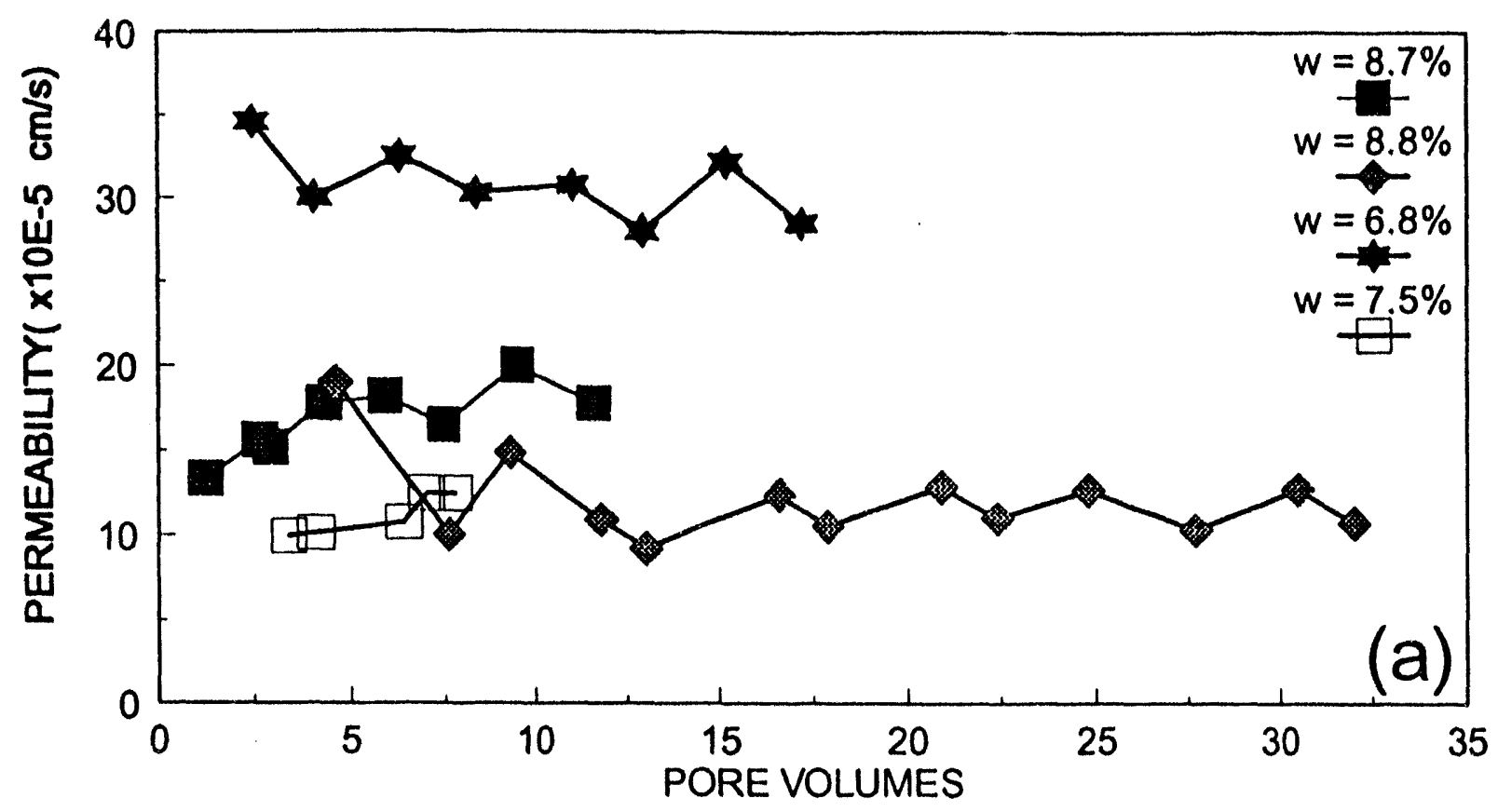

PERMEABILITY vs PORE VOLUMES for $w=6-9 \%$

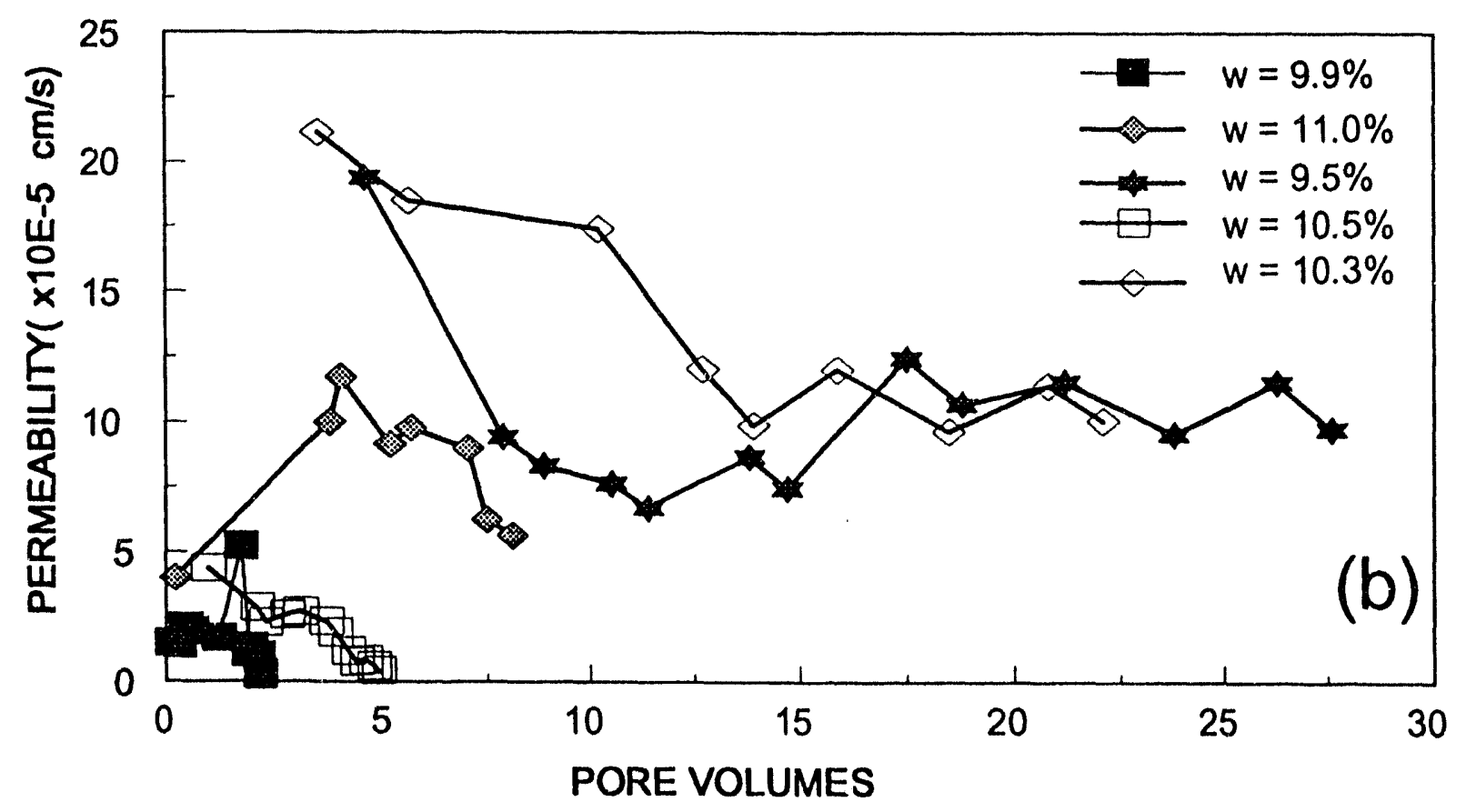

PERMEABILITY vS PORE VOLUMES for $w=9-11 \%$

Figure 7 Results of the Flexible Wall Peremability Tests: a) w range of $6 \%-9 \%$ and b) w range of $9 \%$ to $11 \%$ 


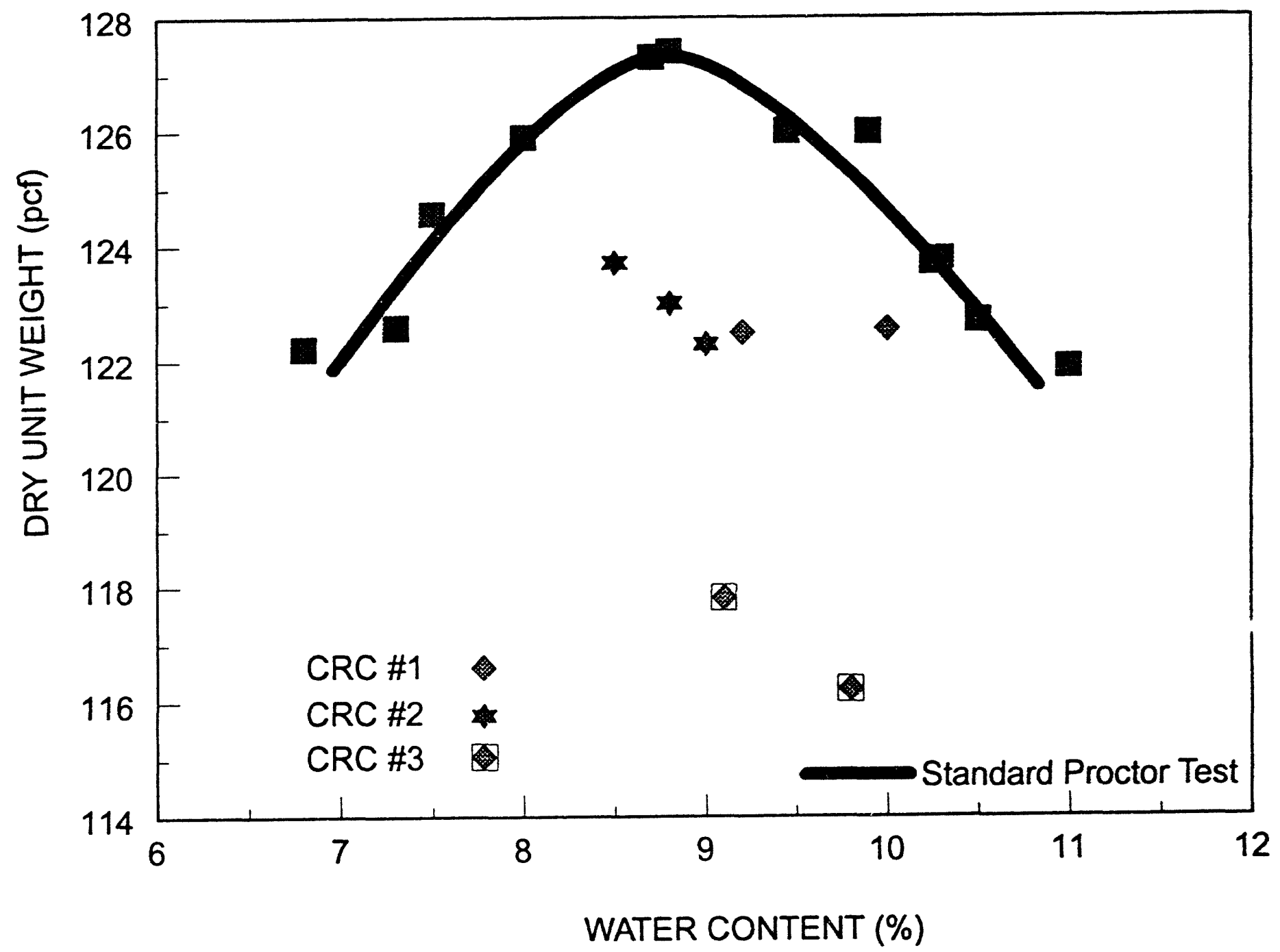

Figure 8..Dry Unit Weight vs Molding Moisture Content 


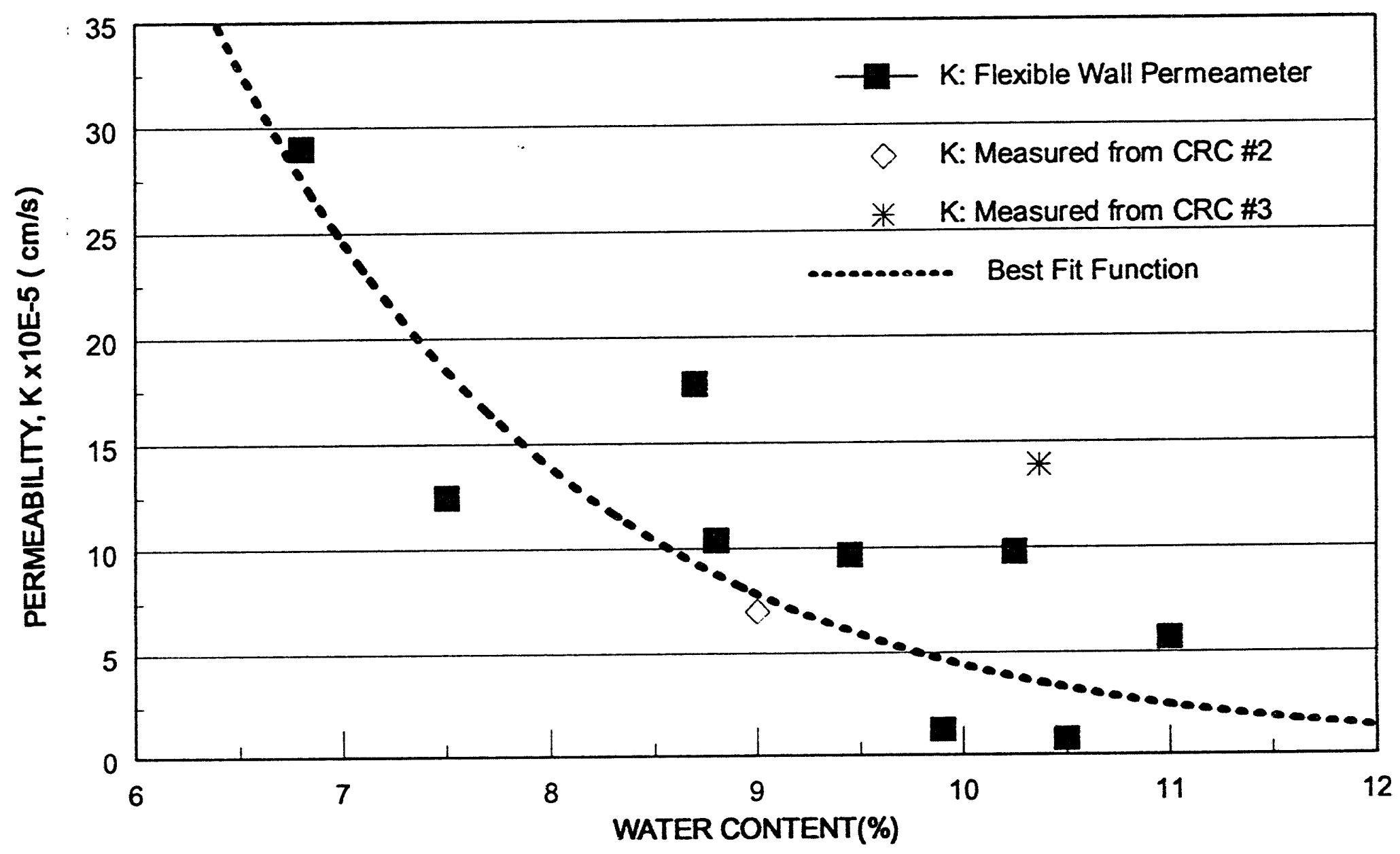

Figure 9. Variation of Peremability as a Function of Molding Water Content (w) 


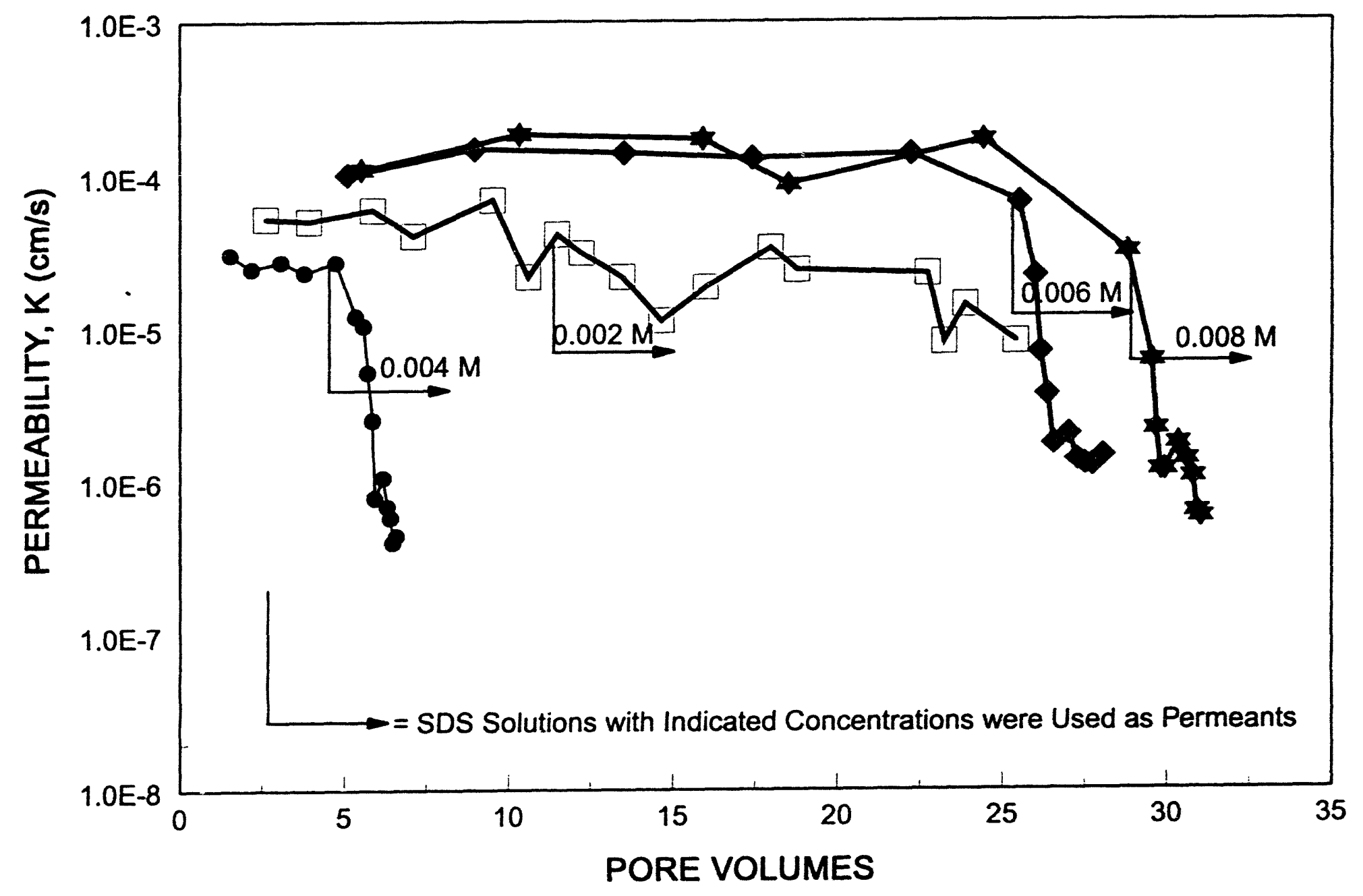

Figure 10. Effect of SDS Surfactant on Measured Permeability 

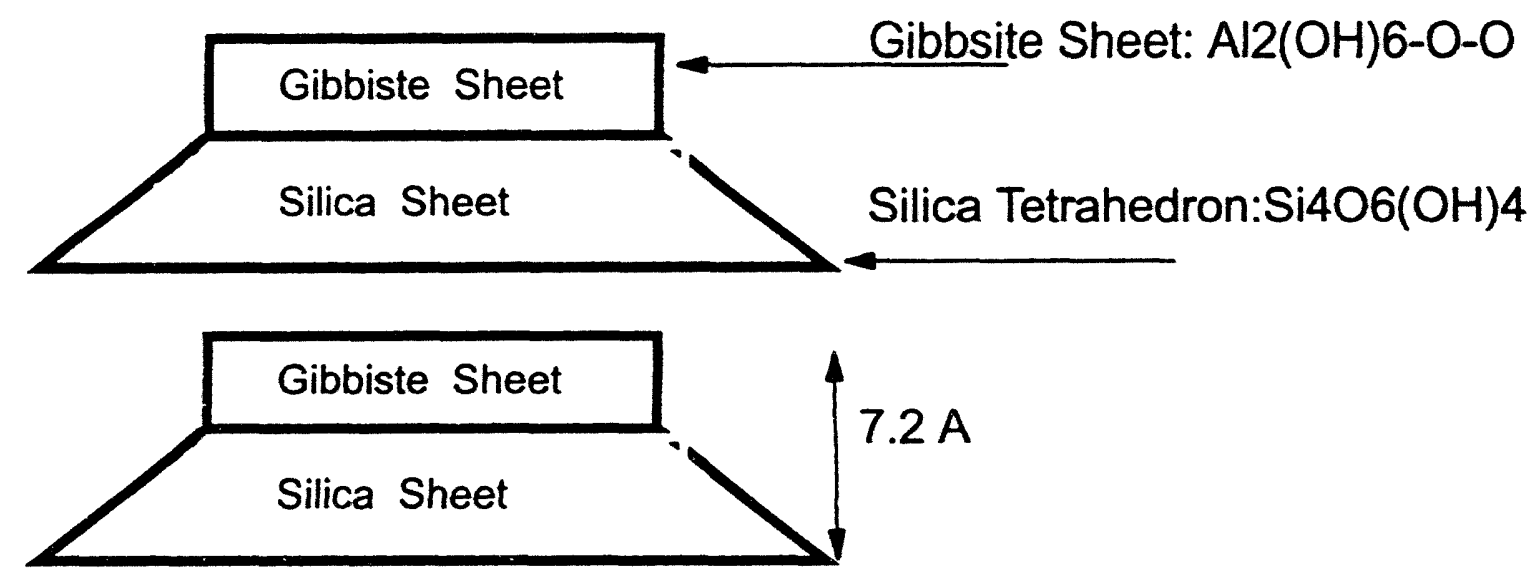

Structural Formula

$(\mathrm{OH})_{8} \mathrm{SI}_{4} \mathrm{AL}_{4} \mathrm{O}_{10}$

Figure IIL Structure of Kaolinite Clay Used in this Study 


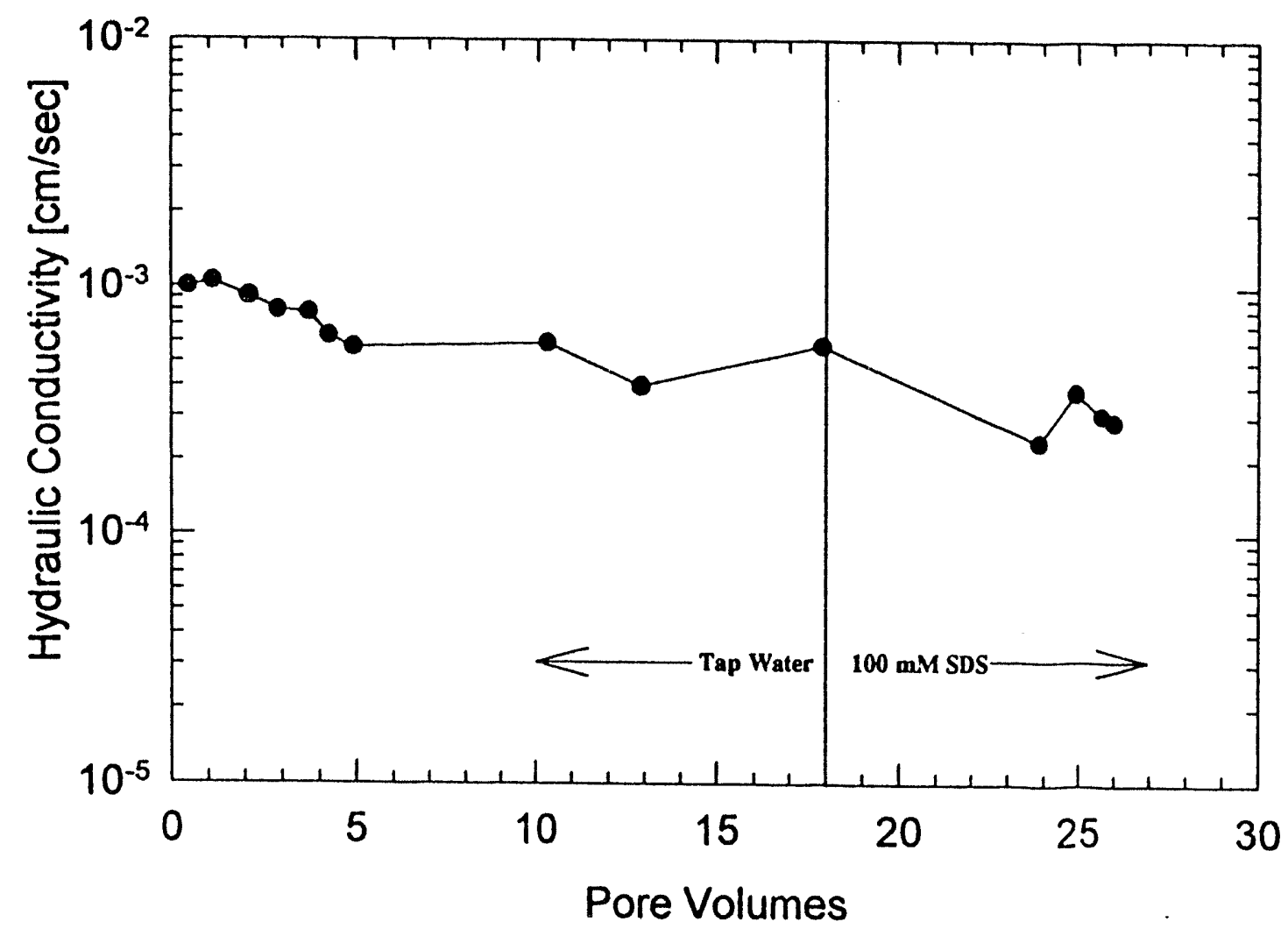

Figure 13. Hydraulic Conductivity as a Function of Permeant Fluis-Ottawa Sand 


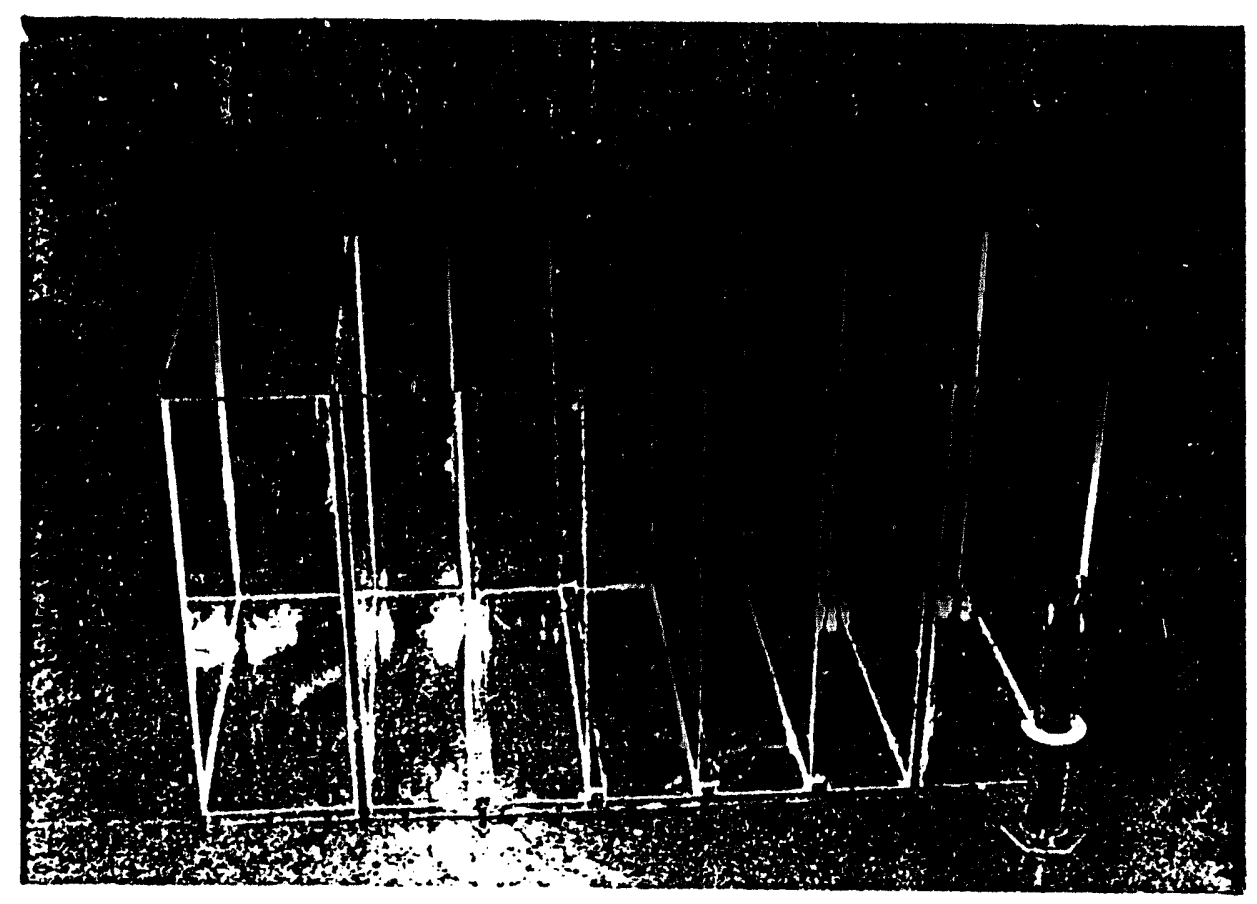

Figure 14. Photograph of the Contaminant Recovery Cell (CRC) 
PLAN

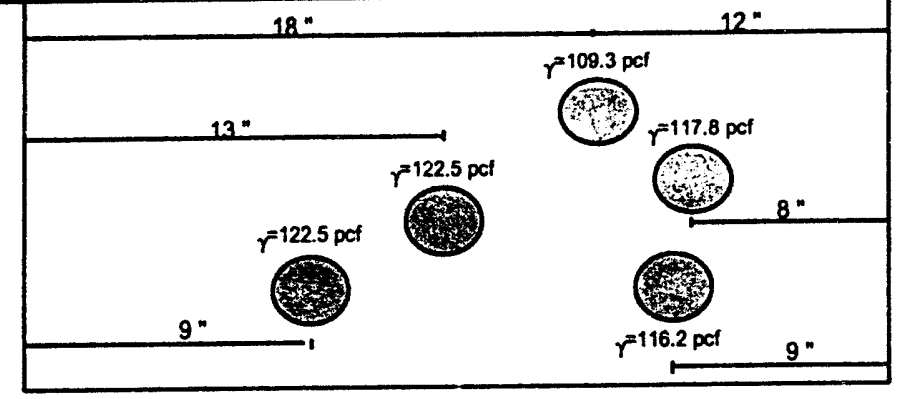

\section{CRC \#1}

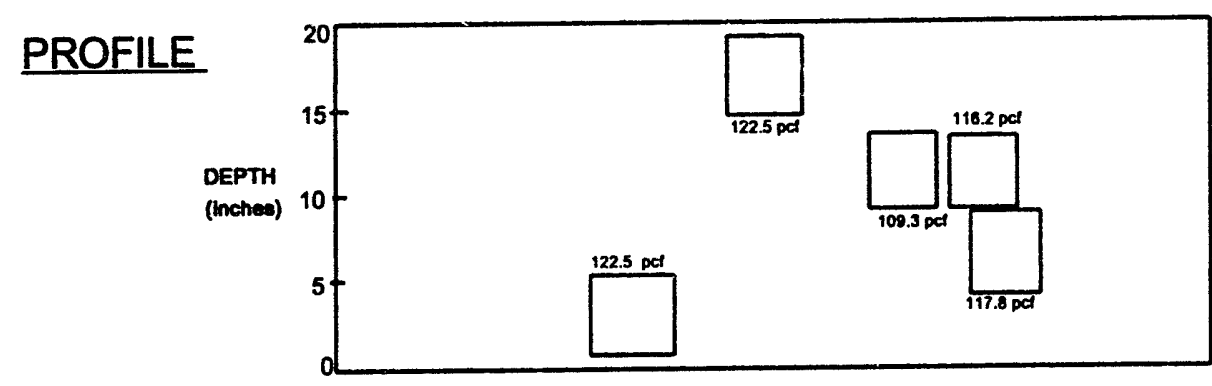

$\pm$ PLAN

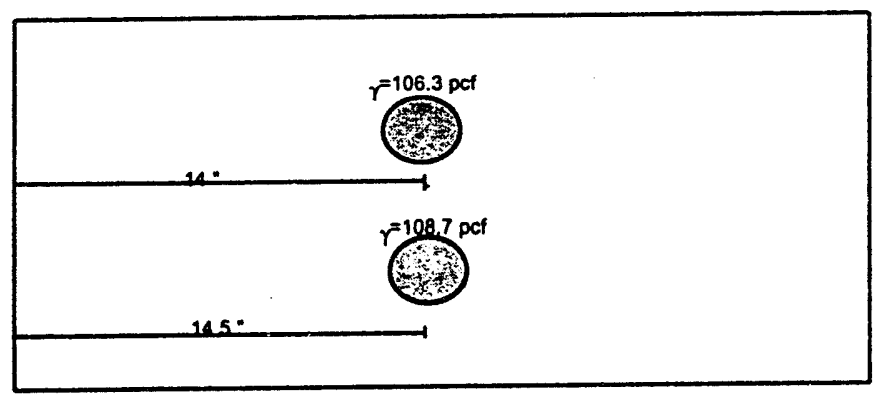

CRC \#3

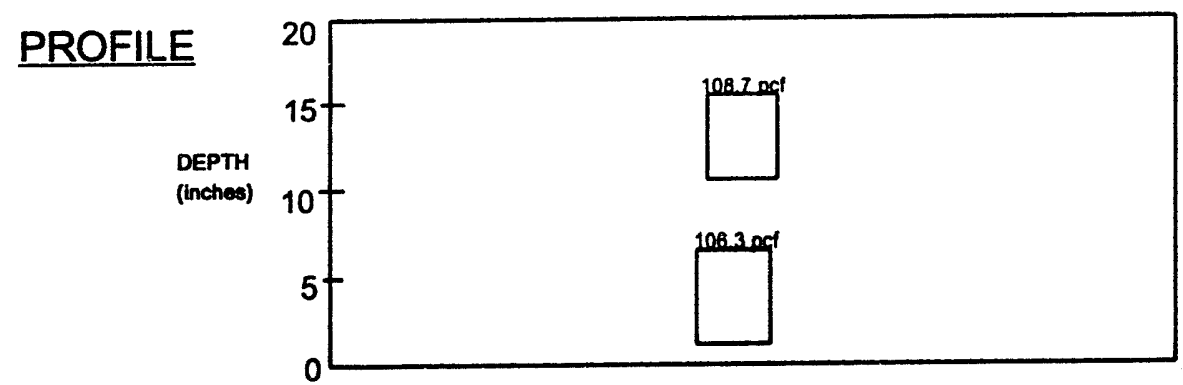

PLAN

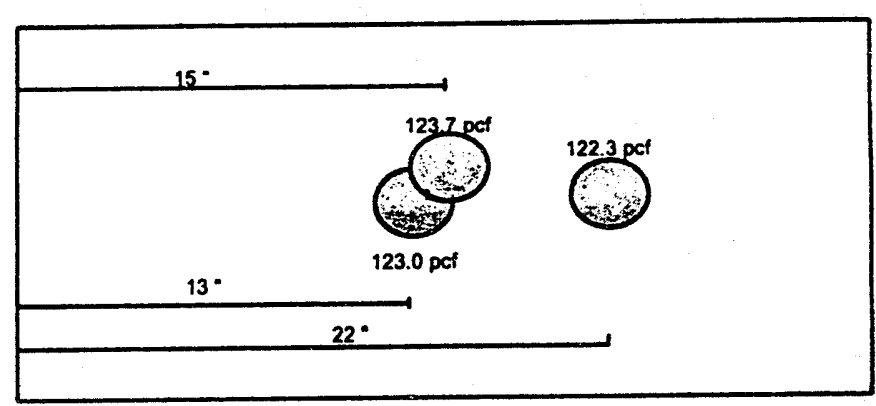

CRC \#2

PROFILE

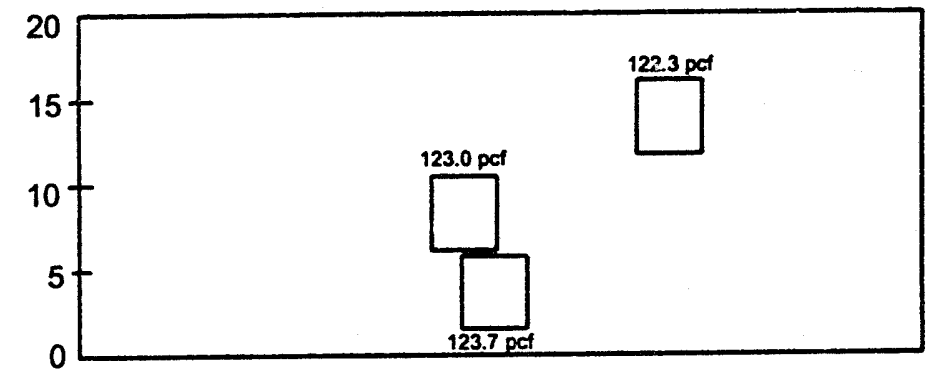

PLAN

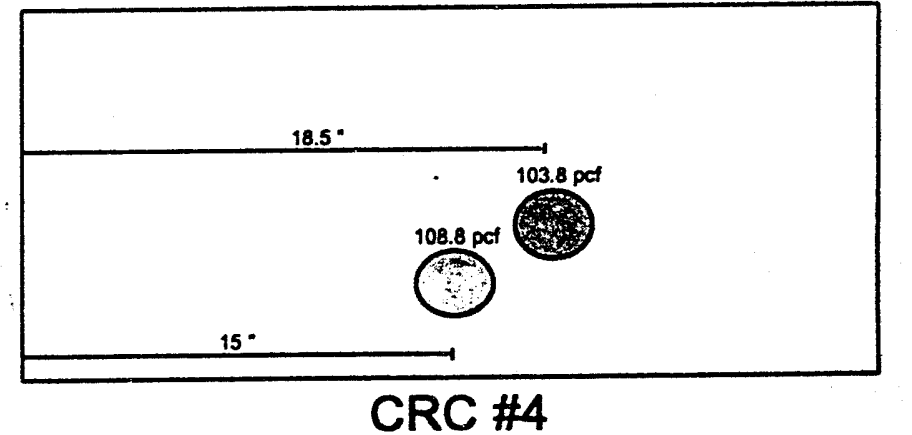

PROFILE

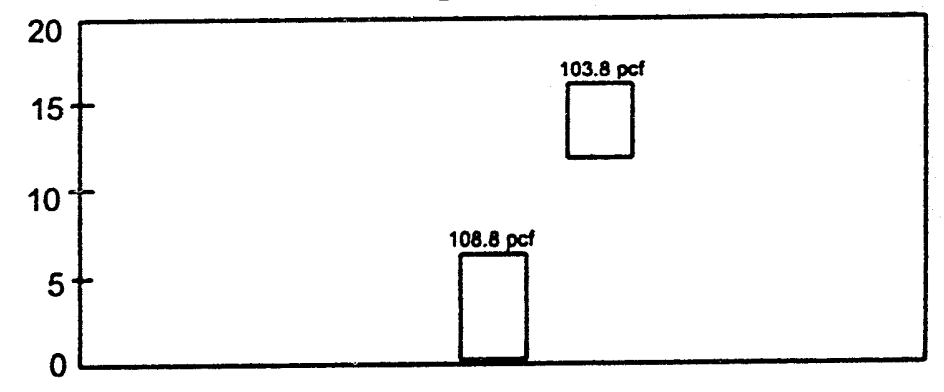

Figure 15. Measured In-Place Densities from Sand Cone Tests 


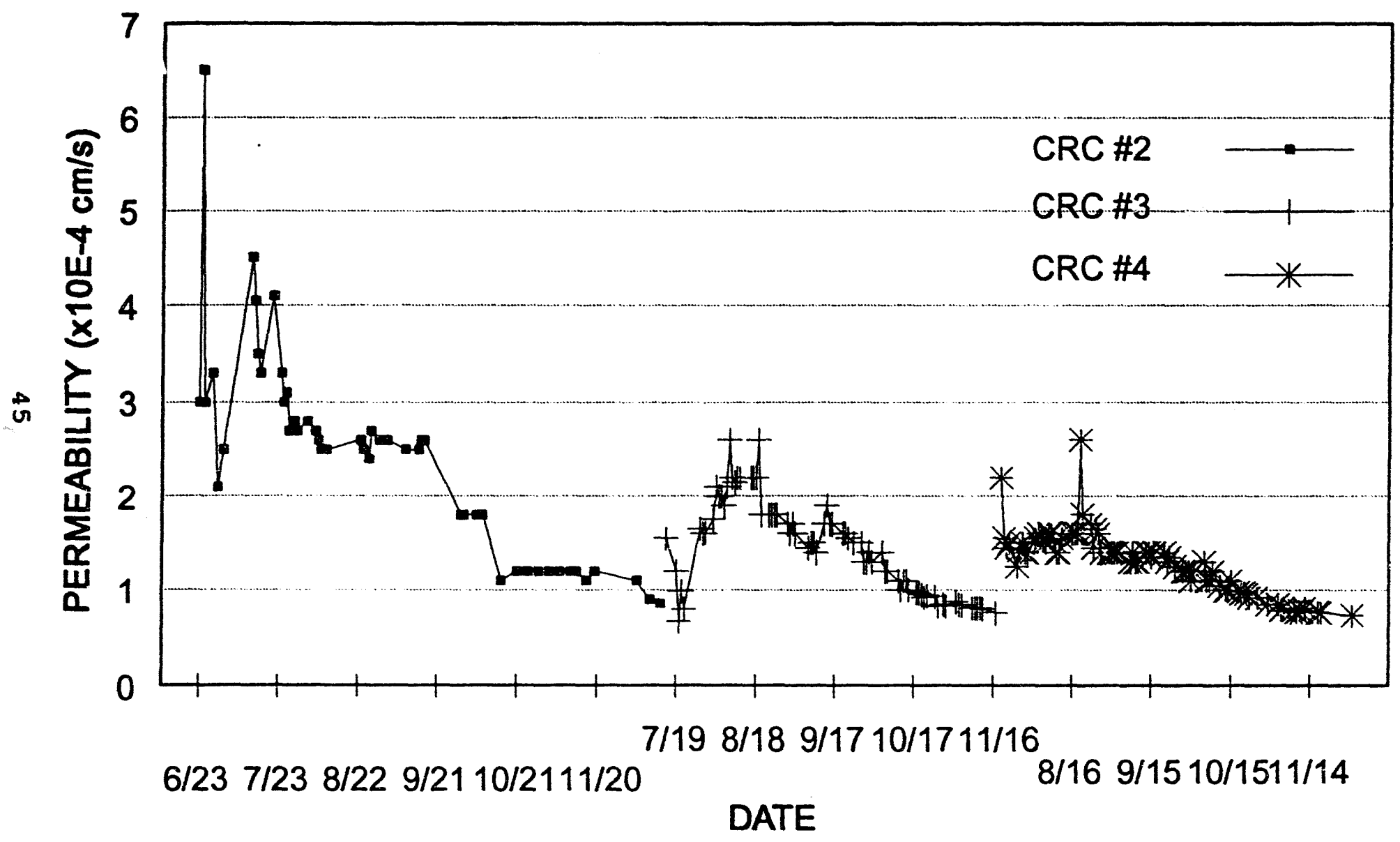

Figure 16. Variation of Hydraulic Conductivity as a function of Time: CRC Tests 


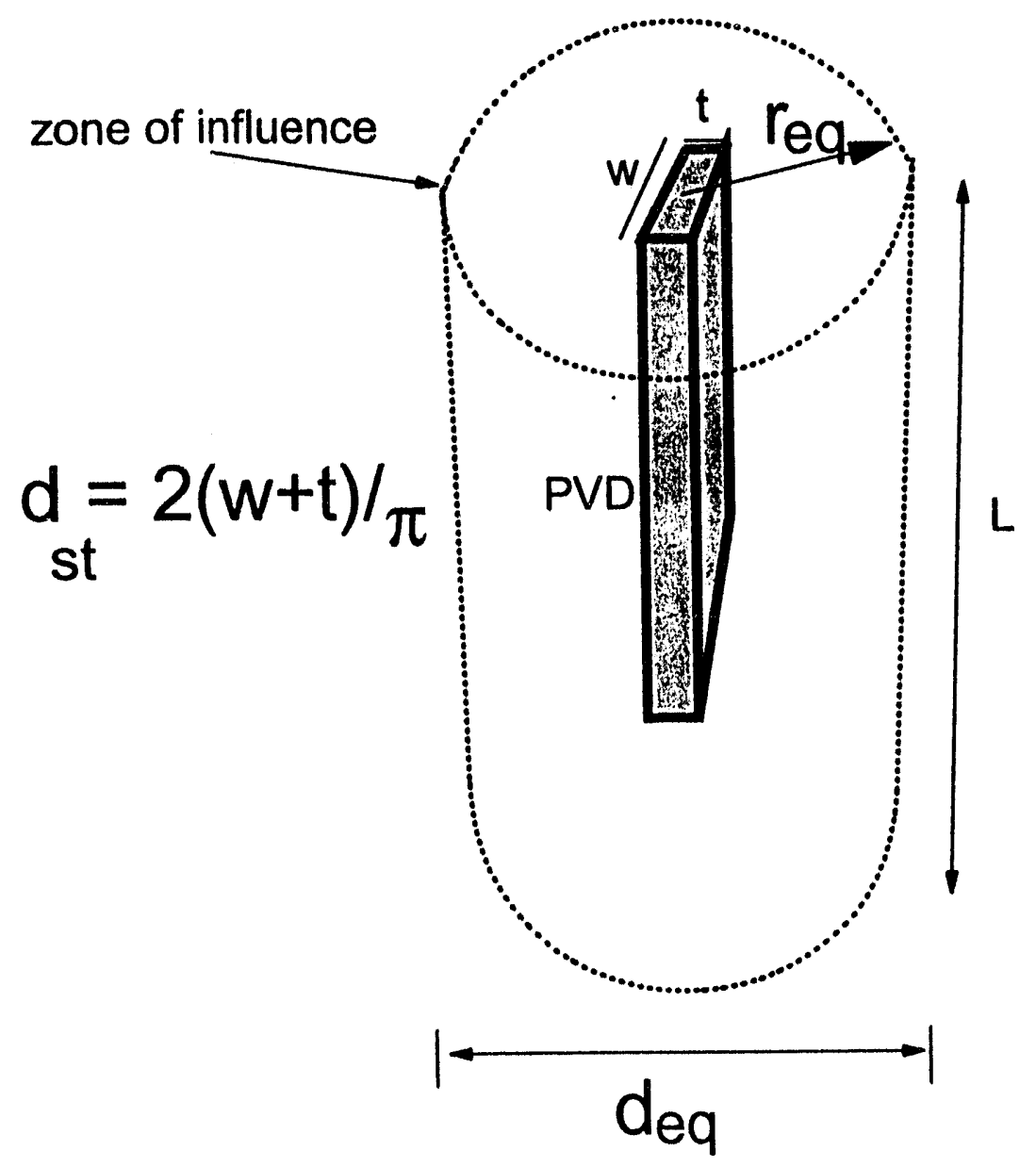

Injection

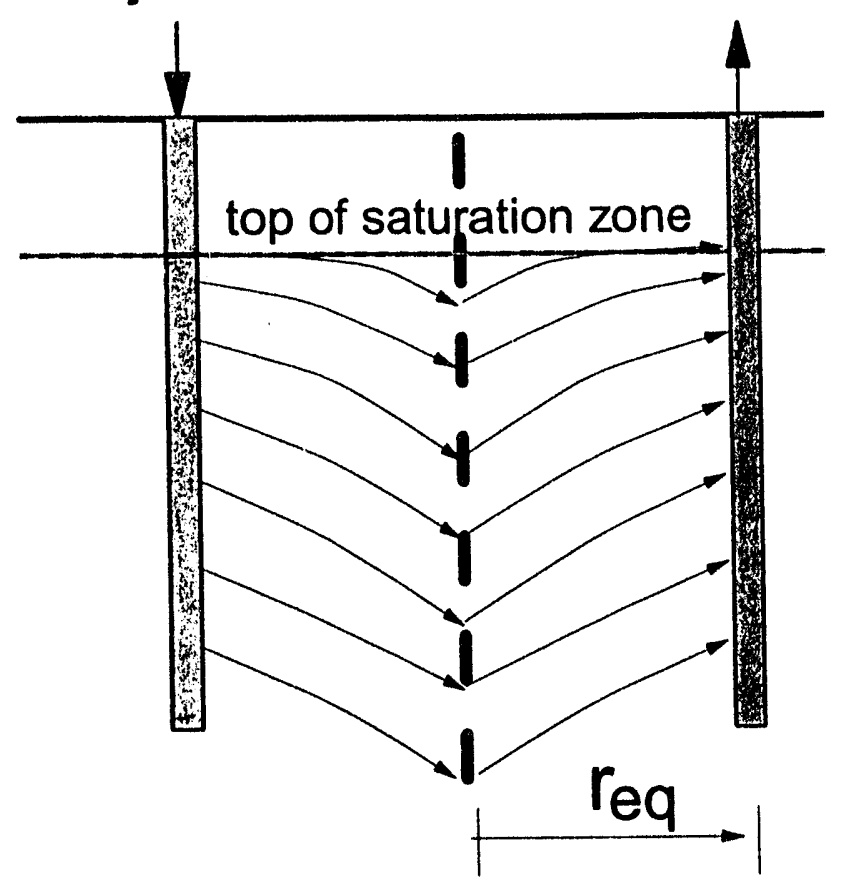

Surcharge Loading

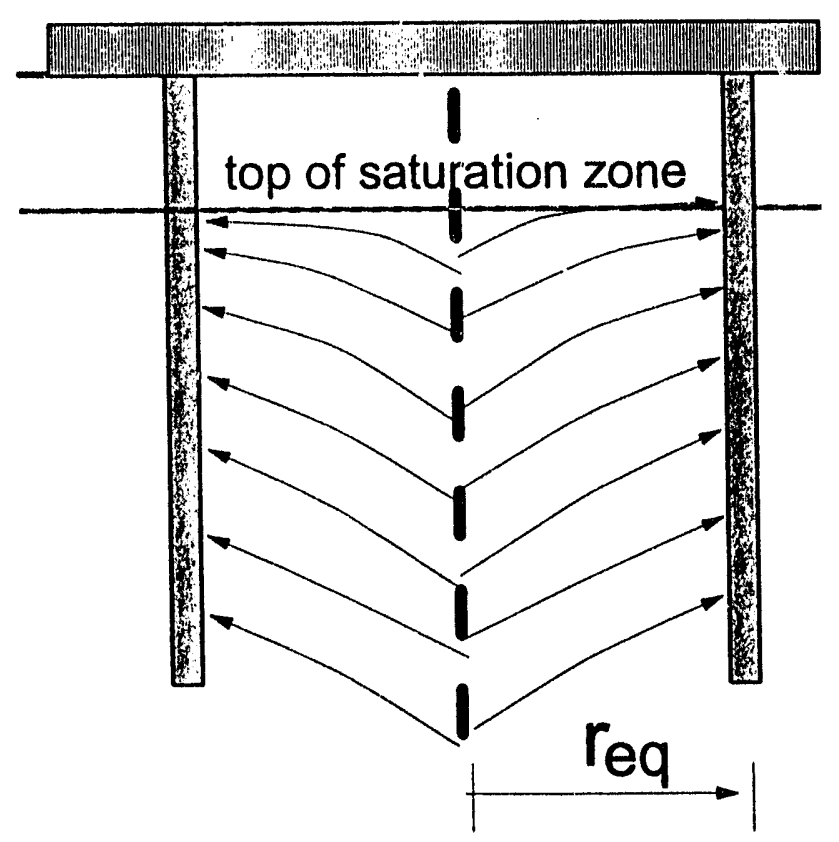

Figure 17. Modeling Configuration for the PVD 

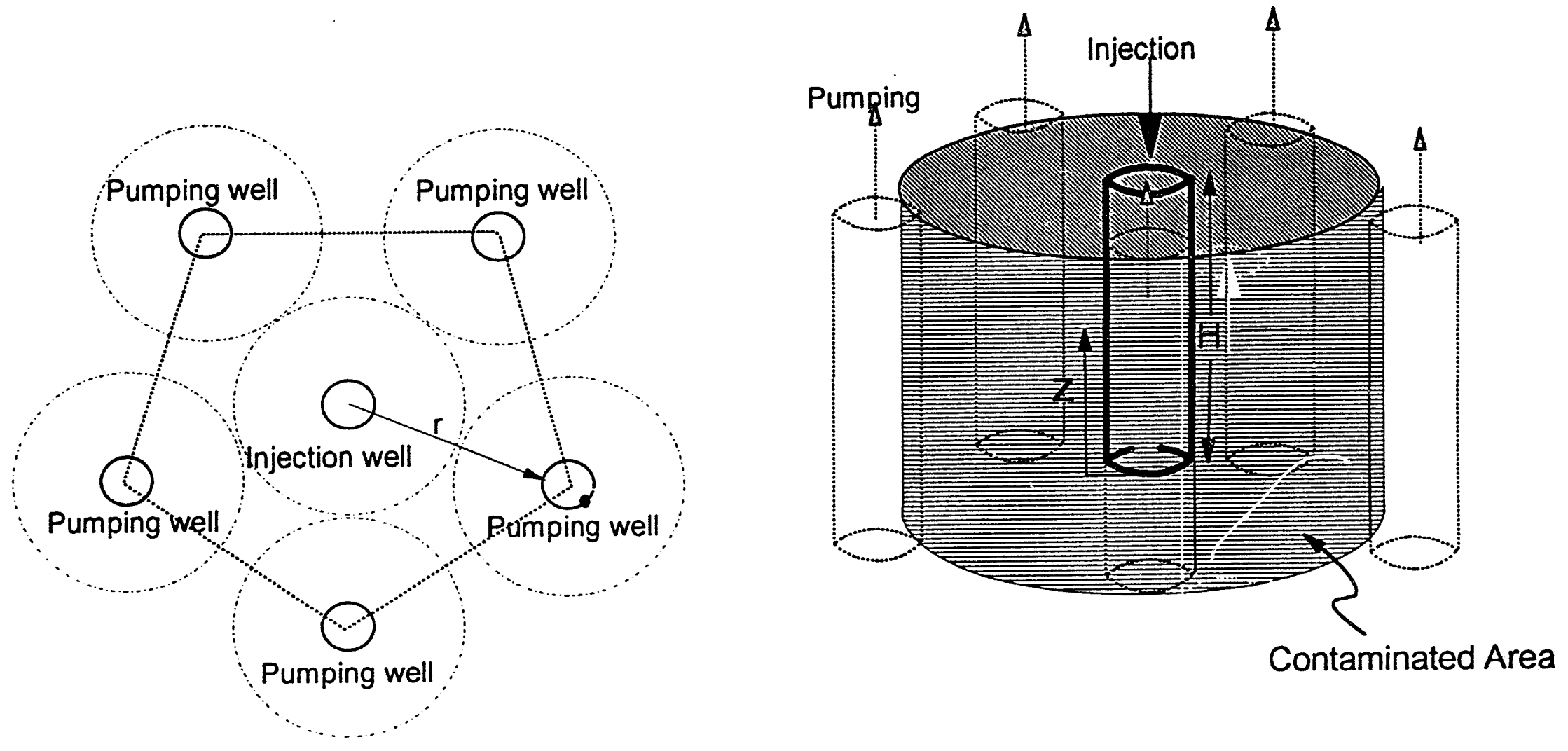

Figure 18. Well pattern and modeling configuration 


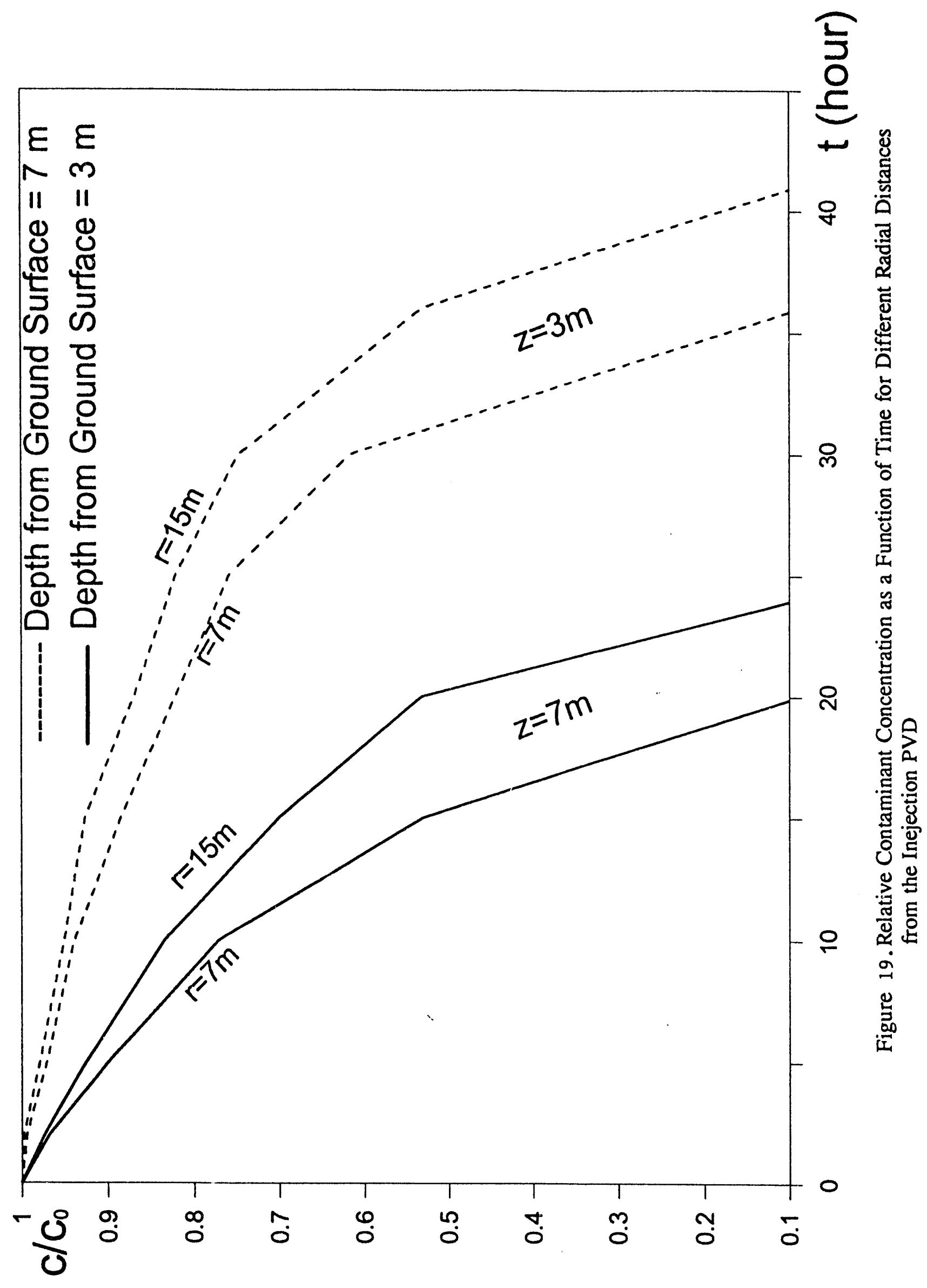


Site Remediation Technologies: In Situ Bio-remediation of Organic Contaminants

(Project MC-2B)

Quarterly Report for Period

for Period October 1 through December 31, 1993

Work Performed Under Contract

No.: DE-FC21-92MC29467

For

U.S. Department of Energy

Office of Fossil Energy

Morgantown Energy Technology Center

Morgantown, West Virginia

By

William A. Sack, Department of Civil Engineering Patrick E. Carriere, Department of Civil Engineering Kevin D. Jones, Department of Civil Engineering Joan E. Cuddeback, Department of Civil Engineering Andrew K. Shiemke, Department of Biochemistry

West Virginia University Morgantown, West Virginia 


\begin{abstract}
In situ bioremediation offers a number of advantages over other processes for destruction of organics in groundwater. Advantages include the potential for complete or near complete destruction of contaminants in place, avoidance of transfer of the pollutants to another medium, less risk of health hazards due to human exposure and cost-effectiveness in many cases. The objective of this research is to evaluate and optimize the ability of methanotrophic, methanogenic, and other selected bacteria for cost-effective biotransformation of a mixture of chlorinated solvents as often found at DOE sites. A five phase workplan is utilized which involves the systematic manipulation of environmental conditions to enhance the rate and extent of biodegradation of the candidate VOC's. It is planned to take advantage of the natural symbiotic relationship between the methanogenic and methanotrophic bacteria so as to promote sequential anaerobic/aerobic mineralization of the chlorinated solvents.
\end{abstract}

As noted last quarter, we have experienced difficulty in reliable measurement of TCE and other $\mathrm{CAH}$ compounds both in the Civil and Environmental Engineering Laboratory (CEEL) and in the Biochemistry Laboratory (BL). Although minor problems remain, we are now able to measure TCE successfully in the CEEL after purchase of a new detector and purge and trap. However, since no DOE funds have been available this quarter, we are still not able to measure TCE in the BL and delays in certain tasks have resulted.

The degradation and toxicity of TCE was monitored using aerobic cultures with a minimum detection limit of $5 \mathrm{ppb}$ during this quarter. The use of control samples ensured that biodegradation is occurring. Toxicity was performed at theoretical concentrations up to $200 \mathrm{ppm}$ on viable cultures of metanotrophs. TCE degradation studies under aerobic conditions will be continued into the next period. Attention will be focussed on the determination of degradation rates and optimization using various primary substrates and hydrogen peroxide.

Two additional anaerobic microcosm studies were carried out this quarter using active samples and water and water and killed culture controls. While additional studies will be required to perform complete mass balances, the results showed some reductive dehalogenation of the TCE. In the next quarter, the microcosm studies will be continued to better establish biodegradation and partioning of the TCE between the gas and liquid phases and sorption on the biological solids.

A glass column for the column studies has been received and is being modified with ports for recirculation and sampling. It is anticipated that the anaerobic column studies will be initiated this quarter. 
TABLE OF CONTENTS

PAGE

$1.0 \quad$ INTRODUCTION $\ldots \ldots \ldots \ldots \ldots \ldots \ldots \ldots \ldots \ldots \ldots \ldots \ldots \ldots \ldots$

2.0 PURPOSE $\ldots \ldots \ldots \ldots \ldots \ldots \ldots \ldots \ldots \ldots \ldots \ldots \ldots \ldots \ldots \ldots$

3.0 BACKGROUND $\ldots \ldots \ldots \ldots \ldots \ldots \ldots \ldots \ldots \ldots \ldots \ldots \ldots \ldots \ldots$

4.0 METHODOLOGY $\ldots \ldots \ldots \ldots \ldots \ldots \ldots \ldots \ldots \ldots \ldots \ldots \ldots, \ldots \ldots \ldots$

Phase 1: Literature Review $\ldots \ldots \ldots \ldots \ldots \ldots \ldots \ldots \ldots \ldots \ldots$

Phase 2: Batch Studies to Evaluate Optimum Concentration of Growth Enhancing Agents in Both Liquid and

Soil Cultures ..........................

Phase 3: Column Studies to Evaluate the Rate and Extent of Degradation of Candidate VOC's .............. 2

Phase 4: Comparison of TCE Biotransformation by Particulate and Soluble Methane Monooxygenase $\ldots \ldots \ldots \ldots 2$

Phase 5: Investigation of the Utilization of the Symbiotic Relationship Between Methangenic and Methanotrophs Bacteria for VOC Degradation Without Addition of Exogenous Methane ....... 2

5.0 RESULTS AND DISCUSSION $\ldots \ldots \ldots \ldots \ldots \ldots \ldots \ldots \ldots \ldots \ldots$

5.1 Aerobic Batch Studies Using Methanotrophs to Evaluate Optimum Concentration of Growth Enhancing Agents in

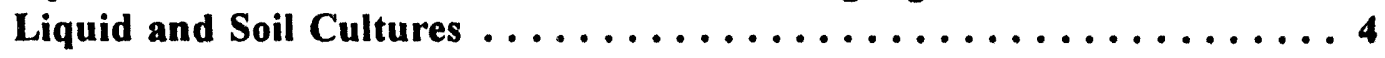

5.1.1 TCE Toxicity Tests $\ldots \ldots \ldots \ldots \ldots \ldots \ldots \ldots \ldots \ldots \ldots 4$

5.1.2 Aerobic TCE Degradation Rate Studies ............. 5 
5.2 Aerobic Batch Studies Using a Mixed Consortia of Methanogens and Other Organisms to Accomplish Reductive

Dehalogenation ...........................8

5.2.1 Gas Chromatography Equipment Modification ..........8

5.2.2 Gas Chromatography Standardization .................

5.2.3 Preliminary Microcosm Studies and General

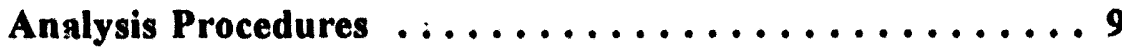

5.2 .4 Microcosm Studies Sets $\# 5$ \& $\# 6 \ldots \ldots \ldots \ldots \ldots \ldots \ldots$

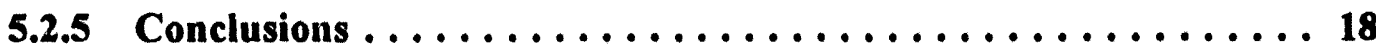

$5.3 \quad$ Research Planned for Next Quarter .................... 19

5.3.1 TCE Degradation Rate Evaluation ................ 19

5.3.2 Evaluation of Reductive Dehalogenation Using

Serum Bottle Microcosms ................. 20

5.3.3 Design and Operation of Columns for VOC

Biotransformation .................... 21

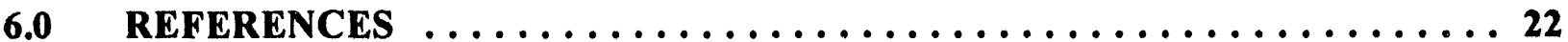

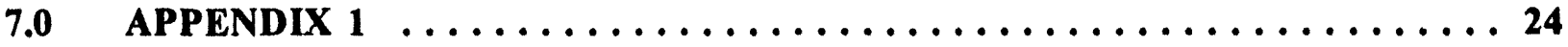

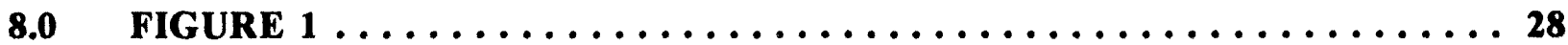




\section{LIST OF TABLES}

Table 1. TCE Concentration Changes in Parts Per Billion for M. capsulatus Cultures and Control Samples $\ldots \ldots \ldots \ldots \ldots \ldots \ldots \ldots \ldots \ldots \ldots \ldots \ldots \ldots$

Table 2. Results of Microcosm Study Set $\# 5 \ldots \ldots \ldots \ldots \ldots$

Table 3. TCE Standardization Results . . . . . . . . . . . . . . . . 16

Table 4. Results of Microcosm Study Set $\# 6 \ldots \ldots \ldots \ldots \ldots \ldots$ 


\subsection{INTRODUCTION}

A variety of toxic organic contaminants are found at DOE sites including fuel hydrocarbons, polychlorinated biphenyls (PCB's) and volatile organic solvents such as trichloroethylene (TCE), perchloroethylene, and carbon tetrachloride. These compounds may occur as single contaminants, but are often found in conjunction with heavy metals or in mixed wastes containing radioactive components.

In-situ bioremediation has a number of advantages for destruction of organic contaminants in groundwater. Other processes such as sorption and volatilization do not destroy contaminants, but rather just concentrate them or transfer them to another medium. Abiotic (chemical) transformation is not normally cost-effective in groundwaters and may even result in production of more toxic chemical species.

Clean-up methods often involve soil flushing to mobilize the contaminants for transport to the surface for treatment. However, because many organic contaminants sorb to soils, they are not readily leached from the soils often leaving toxic residuals in place even after flushing. Furthermore, bringing the contaminants to the surface increases the risk of health hazards due to human exposure. There is increasing recognition that bacteria are present and active in the subsurface and that in situ biotransformation offers a potentially more effective and economical method of contaminant destruction.

\subsection{PURPOSE}

The purpose of the research is to evaluate and optimize the ability of methanotrophic, methanogenic, and other selected bacteria for cost-effective biotransformation of TCE and other volatile organic compounds (VOC's) found at DOE sites. The approach outlined involves the systematic manipulation of environmental conditions in the subsurface for the purpose of enhancing the rate and extent of biodegration of candidate (VOC's). This approach takes advantage of the ubiquity of methanotrophic and methanogenic bacteria and introduction of nonnative organisms should not be necessary. Also, only relatively benign enhancing compounds will be added in non-toxic concentrations. It is envisioned to take advantage of the natural symbiotic relationship between the methanogenic and methanotrophic bacteria so as to promote sequential anaerobic/aerobic mineralization of the chlorinated solvent contaminants.

\subsection{BACKGROUND}

A number of exciting develop,nents are occurring in the field of environmental biotechnology (engineering applications of nirrobial ecology). For example, while trace concentrations of some organic contaminants cannot support microbial growth as the sole electron donor, they can still be biotransformed by engineering the system so that the microbial population obtains the majority of its energy and carbon from a different compound that serves as the primary substrate. This is 
sometimes referred to as secondary utilization. There are also many organic contaminants that are biotransformed in the environment for which no microorganisms have been found which are able to use them as sole carbon source. This is a special case of secondary metabolism often termed cometabolism. Cometabolism has been defined (Dalton, 1982) as the "transformation of a non-growth substrate in the obligate presence of a growth substrate or another transformable compound". As an example, halogenated methanes, ethanes, and ethylenes are poor growth substrates for bacterial growth but may be degraded by methanotrophs growing aerobically on methane (Henson et al., 1988).

\subsection{METHODOLOGY}

The principal focus of the investigation is to develop methods which will enhance bacterial metabolism of organic contaminants in sub-surface environments in order to increase the rate and extent of biodegradation. Our original proposal was organized into five phases, as follows:

Phase 1: Literature Review.

Phase 2: Batch Studies to Evaluate Optimum Concentration of Growth Enhancing Agents in Both Liquid and Soil Cultures.

Phase 3: Column Studies to Evaluate the Rate and Extent of Degradation of Candidate. VOC's.

Phase 4: Comparison of TCE Biotransformation by Particulate and Soluble Methane Monooxygenase.

Phase 5: Investigation of the Utilization of the Symbiotic Relationship Between Methanogenic and Methanotrophic Bacteria for VOC Degradation Without Addition of Exogenous Methane.

A comprehensive literature review of biodegradation of chlorinated solvents was completed and submitted as part of the second quarterly report. As a result of this review we realized the potential for developing the relationship between the methanotrophs (aerobic) and the methanogens (anaerobic) for mineralization of both highly chlorinated and less chlorinated compounds. Emphasis was given to this approach in subsequent phases of the workplan.

The methanotrophs have an obligate requirement for methane, which is complimented by the methane production of methanogenic organisms. Furthermore, the methanogens ability to dehalogenate more oxidized (more halogenated) pollutants, generating vinyl chloride, is complimented by the ability of the methanotrophs to mineralize vinyl chloride and other small mono-chlorinated hydrocarbons. The rate limiting step of mineralization under anaerobic conditions is the dehalogenation of vinyl chloride. Thus, it might be advantageous to induce methanotrophic growth at the point where all (or most) of the more chlorinated compounds have been dehalogenated to vinyl chloride. The number of chlorines dramatically effects the rate of 
anaerobic degradation: the more chlorines the faster the rate of degradation (Sims et al., 1990). A higher number of halogen substituents results in a more oxidized compound making it more susceptible to biological reduction.

Although methanogenic bacteria will not grow in the presence of oxygen, the reductive dehalogenation reaction is somewhat oxygen tolerant. The rate of TCE degradation is reduced under micro-aerophilic conditions, but not completely blocked (Freedman and Gossett, 1989). Under these conditions a suitable source of reducing equivalents must be provided (eg. methanol, hydrogen, acetate, and formate). It is interesting to note in this regard that Kastner (1991) reported that an aerobic enrichment culture's ability to dechlorinate cis-1,2-dichloroethylene was shown to be dependent on a cyclic transition from aerobic to anaerobic conditions and limited oxygen supply. It may also be possible to increase biodegradation rates by alternating methanogenic and methanotrophic growth conditions, thus limiting the accumulation of potentially toxic byproducts, as well as optimizing the alternating production of methane rich and oxygen rich conditions.

\subsection{RESULTS AND DISCUSSION}

In order to accomplish project objectives in a timely manner, the research is being conducted in two complimentary paths as will be discussed in detail in the following sections. Studies to enhance the aerobic degradation of target compounds by methanotrophs are being performed by Shiemke (biochemistry) while Sack and Carriere (environmental engineering) are working on anaerobic reductive dehalogenation using methanogens. Information gained from the separate aerobic and anaerobic research studies will then be combined to optimize sequential anaerobic/aerobic mineralization of the chlorinated solvents.

As noted in Section 5.1 of the previous quarterly report (October 1993), problems have been experienced in measurement of TCE and others CAH compounds both in the Biochemistry Lab (Shiemke) and in the Civil and Environmental Engineering Lab (Sack and Carriere).

Although minor problems remain, we are now able to measure TCE successfully in the Civil and Environmental Lab. As discussed in Section 5.2.1 below, the problem was solved by purchase of a new detector and purge and trap for the GC. It was anticipated that funds would be available from DOE early in the quarter under the new grant to procure the needed equipment and supplies. However no funds were available this quarter and purchase was delayed until funds could be obtained from non-DOE sources. As a result, there was a reduction in the experimental progress planned for this quarter in Civil and Environmental Engineering. It was noted in our last report that the Biochemistry lab had identified the need for a new detector and funds were requested in our proposal for the detector. As noted above, the funds have not yet been received and hence delays have also occurred in the work plan for certain tasks in the Biochemistry Lab. In conclusion, while some tasks have moved forward, tasks requiring measurement of VOC's have been delayed by two to three months in both laboratories. 


\subsection{Aerobic Batch Studies Using Methanotrophs to Evaluate Optimum Concentration of Growth Enhancing Agents in Both Liquid and Soil Cultures}

The goal of our studies of aerobic biodegradation of TCE is to find conditions that enhance the rate of TCE degradation by methanotrophic bacteria. Our approach involves measuring the rate of TCE breakdown in pure cultures of methanotrophic bacteria, under various conditions. We are primarily interested in measuring the effect of compounds that are expected to enhance the metabolic rate of the bacteria. These include compounds that the bacteria can use as sources of reducing equivalents, such as methanol and formate, as well as peroxide as a source of oxygen. We will also investigate the effect of metal chelators that may alter the nature of the oxidative enzymes expressed by the bacteria, to determine which enzymes are most efficient for TCE degradation.

5.1.1 TCE Toxicity Tests Trichloroethylene toxicity tests were performed on cultures of Methylococcus capsulatus in order to determine the limitations for TCE biodegradation rate determinations in the laboratory, as well as the feasibility of bioremediation in an area with high TCE contamination levels. Tests of TCE toxicity were performed identically to the hydrogen peroxide toxicity tests done previously (see our previous Quarterly Report). M. capsulatus cultures were transferred by sterile loop into $125 \mathrm{ml}$ flasks containing $50 \mathrm{ml}$ nitrate mineral salts media, $0.5 \mathrm{ml}$ phosphate buffer, and $0.5 \mathrm{ml}$ stock vitamin solution. The cultures were fed methane at $20 \%$ by volume and placed in an incubated shaker at $40^{\circ} \mathrm{C}$ and $180 \mathrm{rpm}$. Methane was added at $20 \%$ by volume every 24 hours for a period of three days. Contents of the flasks were combined and $10 \mathrm{ml}$ added to culture flasks containing $100 \mathrm{ml}$ nitrate mineral salts solution, $1 \mathrm{ml}$ potassium buffer, $1 \mathrm{ml}$ vitamin solution and $0.05 \mathrm{ml}$ copper sulfate solution. Methane was added to these flasks at $20 \%$ by volume and the flasks were placed in the shaker-incubator at $40^{\circ} \mathrm{C}$ and $180 \mathrm{rpm}$. Methane was added at $20 \%$ by volume every 24 hours. Absorbance readings at $540 \mathrm{~nm}$ were taken periodically using a Bausch and Lomb Spectrophotometer 20. Initial readings, taken after two days growth in the culture flasks showed that two only of five flasks contained viable cultures, so all other flasks were discarded.

TCE was added to one of the remaining flasks at a concentration of $5.0 \mathrm{ppm}$. The second flask was maintained as a control and subjected to the same conditions as the first flask without the addition of TCE. After the addition of TCE, methane was added to each flask at $20 \%$ by volume and the optical density at $540 \mathrm{~nm}$ was measured every five to fourteen hours. After 48 hours, the TCE concentration in the first flask was doubled to $10.0 \mathrm{ppm}$, and again methane was added to each flask at $20 \%$. TCE was added to the first flask at $10.0 \mathrm{ppm}$ every 24 hours for three days and then doubled again. The amount of TCE added to the culture was increased in this manner up to $240 \mathrm{ppm}$, over a period of 23 days. Methane was added at $20 \%$ of the head space after each TCE addition. The resulting growth curves show a decline after the iddition of $20.0 \mathrm{ppm}$, $60.0 \mathrm{ppm}$ and, most significantly, after the second addition of $240 \mathrm{ppm}$. With the exception of the second addition of $240 \mathrm{ppm}$ TCE, all cases of growth decline were followed by a growth increase that maintained the culture at a turbidity matching that of the control flask. The TCE exposed culture never fell below a turbidity of 0.145 after TCE was introduced. This culture did, 
however, show larger fluctuations in turbidity than did the control flask.

This experiment was repeated using a control flask and three sample flasks each receiving either $200 \mathrm{ppb}, 400 \mathrm{ppb}$, or $600 \mathrm{ppb}$ of TCE every 24 to 48 hours for a period of five days. As in the previous experiment, the flasks were fed methane at $20 \%$ by volume every 24 hours and maintained on an incubated shaker at $40^{\circ} \mathrm{C}$ and $180 \mathrm{rpm}$. All three flasks with $M$. capsulatus cultures exposed to TCE did show a growth decline, the extent of which increased only slightly with TCE concentration. The most significant difference between the TCE-exposed cultures occurred only after two consecutive exposures; turbidity for all three flasks declined by approximately 0.02 units while that of the control flask rose by 0.06 units.

Several factors reduce our confidence in the results of this experiment. The concentration of TCE added to the test flasks in this experiment was determined according to the volume of the liquid phase only. The flask headspace was not considered, although it contains a volume greater than $400 \mathrm{ml}$. Headspace consideration and application of the phase partitioning equation mentioned in the previous quarterly report would bring the TCE concentration in the liquid phase to levels well below the nominal concentrations described above. In addition, the flasks were maintained at a temperature of $40^{\circ} \mathrm{C}$, which would cause more extensive transfer of the volatile TCE from the liquid phase into the vapor phase of the flask. The removal of $20 \%$ of this headspace was necessary for each addition of methane. In this way, methane addition also contributed to TCE loss from the aqueous phase. The unavailability of a gas chromatograph for TCE quantitation at the time of this experiment prevented determination of exact TCE concentrations. TCE toxicity tests will be repeated in the future with emphasis on measuring exact aqueous TCE concentrations using the gas chromatograph now operating at the Engineering Sciences Building.

5.1.2 Aerobic TCE Degradation Rate Studies The gas chromatograph (GC) in the Department of Civil and Environmental Engineering is currently the only instrument available for measuring TCE concentration in microbial samples. This GC uses a purge and trap to strip the volatile halogenated compounds from a sample and concentrate them onto a trap that is specific for halogenated compounds. The compounds, including TCE, are desorbed from the trap and separated on a capillary GC column. The compounds are detected by an electrolytic conductivity detector, which is specific for halogenated compounds, and was recommended by several sources as the detector best suited for our application. Each sample analysis requires approximately thirty minutes to complete, so TCE degradation rates were determined within the bounds of this limitation.

The rate of degradation of organic compounds is directly proportional to their concentration (Riser-Roberts, 1992). The thirty minute analysis cycle restricted initial TCE sample concentrations to below the originally planned 5,000-10,000 ppb. Using a Henry's Constant of 0.455 and the phase concentration equation discussed in the October 1993 quarterly report for this preject, an initial liquid phase concentration of $2,000 \mathrm{ppb}$ was chosen to approximate actual field concentrations.

Methylococcus capsulatus cultures were removed from culture plates and inoculated by sterile 
loop into $250 \mathrm{ml}$ flasks containing a growth medium of approximately $50 \mathrm{ml}$ nitrate mineral salts medium (NMS), containing phosphate buffer, vitamins, and copper sulfate. Methane was added to the headspace at $20 \%$ by volume and the flasks were incubated on a shaker at $180 \mathrm{rpm}$ and $40^{\circ} \mathrm{C}$. Methane was fed to these cultures at $20 \%$ by volume approximately every 24 hours for three days until they reached a turbidity of between 0.11 and 0.20 , at $540 \mathrm{~nm}$. Two sets of six bottles each were used to measure TCE degradation rates. The first set included samples (S30, S40) containing $M$. capsulatus cultures that had previously been exposed to TCE during the toxicity tests described above. The second set included samples (S31, S41) containing $M$. capsulatus cultures thit had been killed by the addition of sodium azide. $125 \mathrm{ml}$ amber bottles and Teflon-lined silica septa caps were prepared by autoclaving. $25 \mathrm{ml}$ of sterile NMS, phosphate buffer, vitamin solution and copper sulfate in the same proportions as the growth medium were added to each bottle. The remaining contents of each bottle were chosen according to the type of control or sample desired, as summarized below:

\section{SET 1}

Headspace Controls (C10, C20): $25 . \mathrm{ml}$ - growth medium

$$
\text { 25. } \mathrm{ml} \mathrm{-} \mathrm{micropure} \mathrm{water}
$$

Methanotroph Samples (S10, S20): $25 . \mathrm{ml}$ - growth medium

$$
\text { 25. } \mathrm{ml}-M \text {.capsulatus culture }
$$

TCE-exposed Samples (S30, S40): 25. ml - growth medium

$$
\text { 25. } \mathrm{ml} \text { - exposed } \mathrm{M} \text {. capsulatus }
$$

A syringe was used to deliver $20 \%$ by volume methane to each bottle through the Teflon-lined silica septa immediately after closing and sealing the bottle. The bottles were transferred to the Engineering Sciences Building and $39.53 \mu \mathrm{l}$ of a $2128 \mathrm{ppm}$ solution of TCE in methanol was added to each sample. The bottles were inverted and placed in an arm shaker and incubated at approximately $28^{\circ} \mathrm{C}$. Before injecting TCE into bottle C20, it was discovered that approximately $0.6 \mu \mathrm{l}$ of the $2128 \mathrm{ppm}$ TCE solution had been added to bottle C10. The resulting TCE concentration was out of workable range, and the sample was discarded.

\section{SET 2}

Headspace Controls (C11, C21): $\quad 25 . \mathrm{ml} \quad$ - growth medium

$$
\text { 25. } \mathrm{ml} \text { - micropure water }
$$

Methanotroph Samples (S11, S21): 25. $\mathrm{ml}$ - growth medium

$$
25 . \mathrm{m} 1-\underset{\text { culture }}{\text { - }} \text { capsulatus }
$$




$$
\begin{array}{lll}
25 . \quad \mathrm{ml} & -\quad \begin{array}{c}
\text { capsulatus } \\
\text { culture }
\end{array} \\
.05 \text { gram } & - \text { sodium azide }
\end{array}
$$

Methane was not immediately added to these bottles. Methane was added routinely beginning the fourth day of analysis. The bottles were transferred to the Engineering Sciences Building and $0.841 \mathrm{ml}$ of a $200 \mathrm{ppm}$ solution of TCE in water was added to each bottle. The bottles were inverted and placed in a floor shaker and incubated at approximately $29^{\circ} \mathrm{C}$. The speed of the shaker was not measured.

TCE concentrations were measured by purge and trap GC, using procedures identical to those used in the section on anaerobic TCE degradation studies. Initial concentrations were determined one hour after TCE had been injected into each sample and subsequent concentrations were determined periodically, with the time of TCE exposure being the same for each sample analyzed. The results of the analysis are presented in Table 1.

Table 1. TCE Concentration Changes in Parts Per Billion for $M$. capsulatus Cultures and Control Samples.

\begin{tabular}{|l|l|l|l|l|l|l|l|l||}
\hline Sample & $\begin{array}{l}1 \\
\text { hour }\end{array}$ & $\begin{array}{l}24 \\
\text { hours }\end{array}$ & $\begin{array}{l}48 \\
\mathrm{r} \cdots\end{array}$ & $\begin{array}{l}72 \\
\text { hours }\end{array}$ & $\begin{array}{l}96 \\
\text { hours }\end{array}$ & $\begin{array}{l}144 \\
\text { hours }\end{array}$ & $\begin{array}{l}168 \\
\text { hours }\end{array}$ & $\begin{array}{l}192 \\
\text { hours }\end{array}$ \\
\hline \hline C20 & 3248 & 2473 & 2781 & - & - & 1859 & 1780 & 1985 \\
\hline S10 & 2292 & 1240 & 1384 & - & - & 953 & 937 & 901 \\
\hline S20 & 1455 & 483 & 398 & - & - & $<5$ & $<5$ & $<5$ \\
\hline S30 & 16401 & 15446 & 15858 & - & - & 12150 & 11926 & 7068 \\
\hline S40 & 16708 & 15928 & 16376 & - & - & 11198 & 11387 & 10469 \\
\hline C11 & 2082 & 985 & 942 & 946 & 966 & 975 & - & - \\
\hline C21 & 1195 & 963 & 996 & 957 & 976 & 962 & - & - \\
\hline S11 & 2199 & 1995 & 2014 & 1696 & 1422 & 1206 & - & - \\
\hline S21 & 2100 & 2081 & 1992 & 1673 & 1569 & 1398 & - & - \\
\hline S31 & 1510 & 1459 & 1473 & 1323 & 1465 & 1400 & - & - \\
\hline S41 & 1213 & 1135 & 1177 & 1114 & 1043 & 1023 & - & - \\
\hline
\end{tabular}

The amount of TCE added to the samples was determined according to a Henry's Constant of 0.455 , consistent with that used for the anaerobic degradation studies described in this report. 
However, according to MacKay and Shiu, 1981, only in cases where the compound is present in consistently high concentrations is it possible to establish phase concentrations with accuracy of better than 5 to 10 percent. With the exception of $S 30$ and $S 40,0.25 \mathrm{ml}$ of sample were injected into $5.0 \mathrm{ml}$ of water contained in the purging vessel. The amount of TCE injected was measured relative to the peak area of a control corresponding to $50 \mathrm{ppb}$. Samples S30 and S40 had values out of range of the standard curve, even with sample volumes of $0.05 \mathrm{ml}$. The exact concentration of TCE in the aqueous phase of these two samples is unknown because some amount of TCE was removed with each methane addition, and no GC analysis was available at the time of TCE exposure.

S20 showed complete TCE degradation in a period of less than six days. This was one of the four $M$. capsulatus cultures that were expected to demonstrate complete degradation. S10 was prepared identically to S20 and showed approximately $61 \%$ degradation over a period of eight days. The other two $M$. capsulatus culture samples, $\mathbf{S} 1$ and S12, showed significant degradation only after the third day, corresponding to the time when methane addition began. Killed culture samples S31 and S41 also show a slight decline in TCE concentration that did not change with the addition of methane. It is not certain whether the TCE decline in these samples is the result of bacterial cells that might have survived the addition of sodium azide or some drift in sensitivity of the GC. The dramatic decrease in TCE concentration in the control samples, particularly during the first 24 hours for samples $\mathrm{Cl1}$, is at this time unexplainable. After the initial decrease, the TCE concentration levels off and remains constant. It may be possible that these samples were contaminated.

With few exceptions all chromatograms contained a single peak at a retention time of approximately $4.03 \mathrm{~min}$. Peaks corresponding to TCE concentrations greater than $1400 \mathrm{ppb}$ had a small accompanying peak at a retention time of $\sim 5.65 \mathrm{~min}$. The compound responsible for this peak remains unknown. In addition, chromatograms at 96 hours from samples $\mathrm{C} 11$ and S21 contained new peaks with retention times of less than $4 \mathrm{~min}$. It is possible that these peaks correspond to TCE daughter products. The lesser halogenated or lighter compounds would be expected to have shorter retention times than TCE on the column used for these studies. Standards of trans- and cis-dichloroethylene and vinyl chloride will have to be obtained to determine whether these peaks correspond to products of TCE degradation.

\subsection{Anaerobic Batch Studies Using a Mixed Consortia of Methanogens and Other Organisms to Accomplish Reductive Dehalogenation}

5.2.1 Gas Chromatography Equipment Modification During the past research quarter considerable time was devoted to working out analytical problems in measurement of TCE and other CAH compounds in the Civil and Environmental Laboratory. A complete description of these problems is given in Appendix 1. A number of modifications have been made in order to achieve reliable VOC values. As noted in earlier reports, we have been using a Hewlett Packard Model 5890 Gas Chromatograph with a donated purge and trap (P\&T) apparatus and an electron capture (ECD) detector. This system had been originally recommended by Hewlett Packard for our application. In early November, the ECD was replaced with an electrolytic conductivity 
detector (ELCD) and a new P\&T apparatus (Tekmar LSC 2000). Both the ELCD and the LSC 2000 were purchased with non-DOE funds. The ELCD is more selective for halogenated compounds and is not affected by water vapor from the purge and trap unit). Since the ELCD has been installed, successful analysis of chlorinated aliphatic compounds (CAHs) has been accomplished and additional anaerobic microcosm studies (sets \#5 \& \#6) have been developed and will be discussed below.

5.2.2 Gas Chromatography Standardization Prior to microcosm sample analysis, TCE standardization was performed. A gravimetric primary standard was prepared monthly using pure methanol and pure TCE. A $50 \mathrm{ml}$ volume of primary standard was prepared in a volumetric flask on a certified analytical balance with approximately $2000 \mathrm{ppm}$ of TCE. The primary standard was transferred to a $40 \mathrm{ml}$ amber glass vial with a mininert-valve screw cap and was stored inverted at ambient temperature for a maximum of one month. Freezers were not used for storage during preliminary standardization, but may need to be considered during later work with extremely volatile VOCs, i.e. vinyl chloride. For each day of GC analysis, a secondary standard was prepared using a $10 \mathrm{ul}$ gastight syringe and measured volumes (approximately $9 \mathrm{ul}$ ) of primary standard were added into approximately $2 \mathrm{ml}$ of pure methanol in amber glass screw cap vials with teflon-lined silicone rubber septa. This resulted in approximately $10 \mathrm{ppm}$ of TCE in the secondary standard. By injecting 2.5 to $63 \mathrm{ul}$ of the secondary standard into a $5 \mathrm{ml} \mathrm{Hamilton}$ luer-lok syringe, filled with organic-free water, concentrations spanning 5 to $126 \mathrm{ppb}$ were achieved in the purge vessel. The secondary standard was only used for one day before discarding.

Typically, five concentrations $(5,25,50,100,126 \mathrm{ppb})$ of the standard were analyzed and plotted versus the peak area to develop the standard curve. The slopes of these plots are referred as "response factors" and have units of ppb/(unit area). The concentration of TCE in a $5 \mathrm{ml}$ liquid sample was obtained by using the equation of the line developed during standardization. Generally, a dilution of the original sample was necessary to achieve TCE concentrations that fell within the range of concentrations on the standard surve. A linear relationship between peak area and concentration was not guaranteed for samples concentrations outside the standard curve ccncentration range.

\subsubsection{Preliminary Microcosm Studies and General Analysis Procedures Once TCE} standardization was performed, preliminary microcosm bottles (pre-set \#5) were prepared and analyzed. The preliminary bottles were used to evaluate the necessary sample dilution factors and to investigate the potential of interferences during TCE measurement. In general, all remaining microcosm samples were analyzed by carefully removing the bottles from storage, avoiding unnecessary agitation of liquid phase and headspace gas phase, and withdrawing a liquid sample. Keeping the bottle inverted with liquid in contact with the teflon-lined septa, a gastight syringe was used to withdraw $0.5 \mathrm{ml}$ of the sample through the septa. Next, the $0.5 \mathrm{ml}$ of microcosm liquid was immediately injected into the $5 \mathrm{ml}$ luer-lok syringe containing halogen-free water for a final volume of $5 \mathrm{ml}$, i.e. 1:10 dilution. Finally, the $5 \mathrm{ml}$ luer-lok syringe was fitted 
onto the purge and trap's miniature inert three-way valve and the sample was injected into the fritted purge vessel. Valves were closed and purging of the volatiles began.

Once a single GC analysis was completed, the purge vessel was emptied of the stripped sample and organic-free water was used to flush and clean the purge vessel. After cleaning, the next sample was retrieved and introduced in the same fashion just described. A detailed summary of the purge \& trap, gas chromatograph, detector and integrator operating conditions are as follows;

Purge \& Trap (Tekmar LSC $2000 \mathrm{w} / \mathrm{MCM}$ )

- Standby: $35^{\circ} \mathrm{C}$

- Purge: $8 \mathrm{~min}$., flowrate $\sim 40 \mathrm{ml} / \mathrm{min}$.

- MCM Desorb Cool: $5^{\circ} \mathrm{C}$

- Desorb Preheat: $175^{\circ} \mathrm{C}$

- Desorb: 3 min. @ 180 C

- Bake: 10 min.@190 C

- Bake Gas Bypass: On @ 120 sec. Delay

- Valve: $100^{\circ} \mathrm{C}$

- Mount: $100^{\circ} \mathrm{C}$

- Line: $100^{\circ} \mathrm{C}$

- MCM Bake: $90^{\circ} \mathrm{C}$

- Runs per Sample: 1

Gas Chromatograph (HP 5890 series II)

- Gas Flow

Carrier: $8-10 \mathrm{ml} / \mathrm{min}$

ELCD Make-up: $18-20 \mathrm{ml} / \mathrm{min}$

Hydrogen: $100 \mathrm{ml} / \mathrm{min}$

Total: $130 \mathrm{ml} / \mathrm{min}$

- Inj. A Temp. $=150^{\circ} \mathrm{C}$

- Det. B Temp. $=210^{\circ} \mathrm{C}$

- Oven Temperature Program

Initial Temp: $35^{\circ} \mathrm{C}$

Initial Time: $1 \mathrm{~min}$.

Rate: $10^{\circ} \mathrm{C} / \mathrm{min}$.

Final Temp.: $180^{\circ} \mathrm{C}$

Final Time: $10 \mathrm{~min}$.

Electrolytic Conductivity Detector (OI Corp. Model 4420)

- Operation Mode: P/T

- Detector Temperature: $900^{\circ} \mathrm{C}$

- Filter: Medium 
Integrator (HP 3396)

- Zero: $0,-4.490$

- $A t^{\wedge} 2: 4$

- CHT SP: 0.5

- AR REJ: 1000

- THRSH: 6

- PK WD: 0.04

- Calibration: Multilevel, Linear

In addition to microcosm TCE measurements, active samples from all remaining microcosm experiments were periodically analyzed for gas production and composition in the serum bottle. After liquid samples had been taken for TCE analysis, active sample bottles were set upright with headspace gas in contact with the teflon-lined septa and a $10 \mathrm{ml}$ gastight, pressure-lok syringe was used to sample the headspace gas. The headspace sample was analyzed for gas composition by using the GOW-MAC gas chromatograph previously described in past quarterly reports. Next, a $10 \mathrm{ml}$ Hamilton ground-glass, gastight syringe, fitted with a luer-lok needle bent at 90 degrees was inserted and allowed to be displaced by the volume of gas produced from within the sealed bottle. The syringe plunger was wet with water for lubrication and to form a gastight seal and as the microcosm pressure came to equilibrium with atmospheric pressure a gas production reading was taken by reading the syringe. This reading was added to the volume taken for gas composition analysis and recorded as total gas production for the active sample.

5.2.4 Microcosm Studies Sets \#5 \& \#6 Once general analysis procedures had been established and the preliminary microcosm samples had been successfully analyzed, additional microcosm studies (sets \#5 \& \#6) were planned and begun.

\section{Microcosm Study Set \#5:}

During late November (11/29/93), the fifth set of anaerobic microcosm bottles was set-up to evaluate VOC partitioning and biodegradation rates. Just as with past anaerobic experiments, maintenance reactor liquid was used to inoculate the active sample bottles. The maintenance reactor continues to operate successfully and displays good general system health and an adequate level of methanogenic activity. In addition to active sample bottles, water controls and killed culture controls were also prepared in triplicate and each of the serum bottles contained the following; 


\title{
Active Samples: $100 \mathrm{ml}$ Maintenance Reactor Liquid \\ $50 \mathrm{mg} / \mathrm{l}$ Methanol \\ $50 \mathrm{mg} / \mathrm{l}$ Acetate \\ $\sim 0.75$ to $1.0 \mathrm{mg} / \mathrm{l} \mathrm{TCE}$
}

\author{
Water Controls: $100 \mathrm{ml}$ Purged D.I. Water \\ $\sim 0.75$ to $1.0 \mathrm{mg} / \mathrm{TCE}$
}

\author{
Killed Controls: $100 \mathrm{ml}$ Maintenance Reactor Liquid \\ (Autoclaved for $30 \mathrm{~min}$. $9250^{\circ} \mathrm{F} \& 15 \mathrm{psi}$ ) \\ $\sim 0.75$ to $1.0 \mathrm{mg} / 1 \mathrm{TCE}$
}

As discussed in the last quarterly report, killed culture controls were added to the experiments in order to evaluate sorption of the contaminant to the biomass. However during the first stages of the fifth microcosm study no attempts were made to evaluate the mass of contaminant sorbed to the biomass. The killed culture controls were sampled and analyzed to merely compare with the water controls. In future microcosm sampling and analysis, plans are being made to use a filtering syringe apparatus to separate the killed biomass before analysis and therefore determine the mass of contaminant which is partitioned onto the biomass as compared to water controls. This will allow sorption coefficients to calculated and an evaluation of potential active sample contaminant partitioning onto the biomass.

During microcosm study set \#5, stock solutions of methanol (100,000 ppm), acetate (100,000 $\mathrm{ppm})$, and TCE (2128 ppm) were prepared with nitrogen purged organic free water. Serum bottles used in the fifth microcosm study were sealed with aluminum crimp caps and teflon lined septa. The fifth set of microcosm bottles were labeled, S5A, S5B, S5C (Samples, Set\#5, Bottle A, B, \& C), C5A, C5B,C5C (Controls, Set \#5, Bottle A, B, \& C), K5A, K5B, K5C (Killed Control, Set \#5, Bottle A, B, \& C) and stored in the dark at $25^{\circ} \mathrm{C}$, under quiescent conditions, with the liquid in contact with the septa.

The fifth set of microcosm bottles were analyzed after approximately 1 hour (after preparation) to obtain initial liquid TCE concentrations. The bottles were then stored and analyzed again after two days. On the second day, after analysis, the active bottles received additional organic substrate ( $50 \mathrm{mg} / \mathrm{l}$ methanol and $50 \mathrm{mg} / \mathrm{l}$ acetate) by using a syringe to inject the substrates through the sealed septa. The active samples did not receive additional TCE on the second day since biodegradation was not apparent. The microcosm bottles were stored and analyzed on day 4 of the experiment. Similar results were obtained for each of the bottles and TCE biodegradation was still not apparent. On day 5, bottles A \& B of the water controls, the killed controls and the active samples were opened and refed. As discussed in past quarterly reports, during refeeding of the microcosms the bottles are opened and maintained anaerobic by sparging 
with oxygen-free gas until sealed. They are fed fresh basal salts medium plus the organic substrates $(50 \mathrm{mg} / \mathrm{l}$ methanol and $50 \mathrm{mg} / \mathrm{l}$ acetate) and finally respiked with the contaminant $(0.75$ - $1.0 \mathrm{mg} / \mathrm{TCE}$ ). Bottle $\mathrm{C}$ from the water controls, the killed controls and active samples was not opened, but instead the active sample, S5C, was given organic substrates $(50 \mathrm{mg} / 1 \mathrm{methanol}$ and $50 \mathrm{mg} / \mathrm{l}$ acetate) by syringe and the "C" bottles (C5C, K5C \& S5C) were stored undisturbed until the time of the next analysis.

Each of microcosm bottles were again analyzed and maintained on the 7 th, 9 th $\&$ 16th day of the fifth microcosm experiment. The bottles were maintained by using a syringe to introduce organic substrates and were not opened after the day 5 refeeding. Gas chromatography results have been collected and are summarized below in Table 2 . The fifth set of microcosm bottles continue to be maintained on a two day cycle and will be evaluated in the future by project personnel. 
Table 2. Results of Microcosm Study Set \#5

\begin{tabular}{|c|c|c|c|c|}
\hline Sample & Day & $\begin{array}{l}\text { TCE } \\
(\mathrm{ppb})\end{array}$ & $\begin{array}{l}\text { Gas Produced } \\
\text { (ml) }\end{array}$ & $\begin{array}{l}\text { Methane Composition } \\
(\%)\end{array}$ \\
\hline$C 5_{A B C}$ & 0 & 798 & & \\
\hline$\overline{\mathrm{K} 5_{\mathrm{ABC}}}$ & "1 & 837 & & \\
\hline$S S_{A B C}$ & 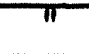 & 761 & - & - \\
\hline & & & & \\
\hline $\mathrm{C5} 5_{\mathrm{ABC}}$ & 2 & 873 & & \\
\hline $\bar{K} 5_{A B C}$ & " & 869 & & \\
\hline$S 5_{A B C}$ & $\pi$ & 795 & 20 & 17 \\
\hline $\mathrm{CS}_{\mathrm{ABC}}$ & 4 & 923 & & \\
\hline$\overline{\mathrm{K} 5_{\mathrm{ABC}}}$ & " & 917 & & \\
\hline $\mathrm{SS}_{\mathrm{ABC}}$ & " & 810 & 12 & - \\
\hline C5 & & & & \\
\hline $\mathrm{CS}_{\mathrm{A}}$ & 5 & 1041 & & \\
\hline $\bar{K} 5_{A}$ & 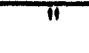 & 1062 & & \\
\hline$S 5_{A}$ & & 949 & - & - \\
\hline $\mathrm{C} 5_{A R}$ & 7 & 1018 & & \\
\hline$\overline{K 5} 5_{A B}$ & " & 1050 & & \\
\hline$\widehat{S 5_{A B}}$ & $\pi$ & 937 & 24 & $\overline{18}$ \\
\hline $\mathrm{C} 5_{\mathrm{c}}$ & 7 & 980 & & \\
\hline$\overline{\mathrm{K} 5_{\mathrm{c}}}$ & $"$ & 973 & & \\
\hline $55_{c}$ & $\pi$ & 828 & 12 & 30 \\
\hline & & & & \\
\hline $\mathrm{C} 5_{\mathrm{AB}}$ & 9 & 1062 & & \\
\hline $\bar{K} 5_{A B}$ & " & 1077 & & \\
\hline$S 5_{\mathrm{AB}}$ & $\pi$ & 985 & - & - \\
\hline & & & & \\
\hline $\mathrm{CS}_{\mathrm{c}}$ & 9 & 1031 & & \\
\hline $\mathrm{KS}_{\mathrm{c}}$ & " & 1082 & & \\
\hline $55_{c}$ & $\pi$ & 822 & $=$ & - \\
\hline $\mathrm{CS}_{\mathrm{AB}}$ & 16 & 998 & & \\
\hline $\mathrm{K} 5_{\mathrm{AB}}$ & & 1020 & & \\
\hline$S 5_{\mathrm{AB}}$ & & 869 & - & - \\
\hline
\end{tabular}




\begin{tabular}{|l|c|c|c|c|}
\hline & & & & \\
\hline $\mathrm{C} 5_{\mathrm{c}}$ & 16 & 986 & & \\
\hline $\mathrm{K} 5_{\mathrm{c}}$ & 1 & 941 & & \\
\hline $\mathrm{S} 5_{\mathrm{c}}$ & 1 & 773 & - & \\
\hline
\end{tabular}

C5 $5_{\mathrm{ABC}}$ - Average of Microcosm Water Control Bottles A, B, \& C $\mathrm{K} 5_{\mathrm{ABC}}$ - Average of Microcosm Killed Control Bottles A, B, \& C $S 5_{A B C}$ - Average of Microcosm Active Sample Bottles A, B, \& C $\mathrm{CS}_{\mathrm{AB}}$ - Average of Microcosm Water Control Bottles A \& B

$\mathrm{K} 5_{\mathrm{AB}}$ - Average of Microcosm Killed Control Bottles A \& B

$S 5_{A B}$ - Average of Microcosm Active Sample Bottles A \& B

$\mathrm{Cs}_{\mathrm{C}}$ - Microcosm Water Control Bottle C

$\mathrm{K} 5_{\mathrm{C}}$ - Microcosm Killed Control Bottle C

$\mathrm{S5}_{\mathrm{C}}$ - Microcosm Active Sample Bottle C

The collected results do provide some useful information, but first GC difficulties must be taken into account and then relative trends can be identified and explained.

As alluded to above, gas chromatography difficulty (sensitivity drift) was experienced during the analysis of set \#5 microcosm bottles. As discussed, TCE standardization was performed prior to sample analysis and immediately before samples were analyzed standards were run and compared to previous results. While reproducibility of the standardization results was within $\sim 5 \%$ at the time of standard curve preparation and an excellent correlation coefficient ( 0.998 or better) could be obtained, over time the GC equipment seemed to consistently provide increases in the responses for the same standard concentrations. For example, Table 3 displays the results obtained during three different standardizations. 
Table 3. TCE Standardization Results

\begin{tabular}{|c|c|c|c|}
\hline Date & $\begin{array}{l}\text { TCE Concentration } \\
(\mathrm{ppb})\end{array}$ & $\begin{array}{c}\text { Response } \\
\text { (area units) }\end{array}$ & $\begin{array}{c}\text { Difference } \\
(\%)\end{array}$ \\
\hline \multirow[t]{4}{*}{$11 / 29 / 93$} & 5 & 78,359 & \\
\hline & 25 & 328,303 & \\
\hline & 50 & 552,931 & \\
\hline & 100 & $1,044,973$ & \\
\hline & & & \\
\hline \multirow[t]{5}{*}{$12 / 02 / 93$} & 5 & 90,946 & 14 \\
\hline & 25 & 436,245 & 25 \\
\hline & 50 & 809,734 & $\overline{32}$ \\
\hline & 100 & $1,436,008$ & 27 \\
\hline & 126 & $1,733,553$ & - \\
\hline \multirow[t]{5}{*}{$12 / 04 / 93$} & 5 & 109,952 & 17 \\
\hline & 25 & 478,982 & 9 \\
\hline & 50 & 878,891 & 8 \\
\hline & 100 & $1,576,633$ & 9 \\
\hline & 126 & $1,871,759$ & 7 \\
\hline
\end{tabular}

Corrections were made for the sensitivity drift and for any given day of analysis. The results from Table 3 indicate that in time the previous standard curves could not be used without introducing an error of approximately 7 to $32 \%$.

Microcosm sample response, even with corrections made, seemed to provide results that increased with time for each sample analyzed (with the exception of results obtained for day 7 bottles $\mathrm{A}$ \& B, day 9 bottle S5C and day 11 bottles A, B \& C). Results that may be more or less expected would include for the control bottles (water \& killed) to stay at the same TCE concentration or experience minor volatile losses due to leaks, while the active sample would remain the same or decrease significantly in TCE concentration as biodegradation takes place. One explanation of the results would be the error which was introduced due to the identified sensitivity drift of the GC. Since each of the triplicate bottles responses consistently agreed, it is believed that the concentrations did not necessarily increase as shown, but were merely high due to the GC sensitivity drift. Even though the GC difficulties were experienced, useful information can still be gained from the set \#5 results.

Microcosm study set \#5 results do indicate initial TCE concentrations that agree with what was expected (taking into account how much contaminant was added and the effects of partitioning). The expected initial TCE liquid concentration was approximately $886 \mathrm{ppb}$ (using the mass balance relationship discussed in the last quarterly report and a dimensionless Henry's Constant 
of 0.455 ). Results reveal somewhat lower initial TCE concentrations for control samples. These concentrations indicate that complete partitioning had probably not been experienced after the first 1 hour period, but day 2 results were close to the concentrations expected for the controls. The later control results (day 4 and beyond) were higher than expected, but this is attributed to the sensitivity drift problem and is not felt to be significant in a relative sense when evaluating the data. All of the recorded results reveal lower TCE concentration in the active microcosm samples. The initial (day 0) active sample TCE concentration was lower than the controls which may explain the overall trend of lower concentrations, however the relative decrease in concentrations was significantly greater in active samples especially during the latest (days 9 \& 16) results recorded. In addition to the decrease of TCE in the active sample bottles, gas production and an increase in methane composition was experienced during the fifth microcosm study. The gas production and composition results indicate anaerobic activity was occurring in the active sample bottles and the reductive dehalogenation process should follow. Results for microcosm study set \#5 indicate that the anaerobic biodegradation process is occurring and it will be interesting to see how later results compare and if signs of degradation products become evident during future TCE analysis.

\section{Microcosm Study Set \#6:}

In addition to microcosm study set \#5, a sixth set of microcosm bottles were set-up and analyzed during the past research quarter. The sixth microcosm study began on 12/10/93 and the serum bottles were prepared identically to the fifth set as noted below:

Active Samples: $100 \mathrm{ml}$ Maintenance Reactor Liquid
$50 \mathrm{mg} / \mathrm{l}$ Methanol
$50 \mathrm{mg} / \mathrm{l}$ Acetate

Water Controls: $100 \mathrm{ml}$ Purged D.I. Water $\sim 0.75$ to $1.0 \mathrm{mg} / \mathrm{TCE}$

Killed Controls: $100 \mathrm{ml}$ Maintenance Reactor Liquid

(Autoclaved for $30 \mathrm{~min}$. $250^{\circ}$ F \& 15 psi)

$\sim 0.75$ to $1.0 \mathrm{mg} / \mathrm{TCE}$

Microcosm study set \#6 was intended to serve as a check for the fifth study and therefore experimental procedures and analytical techniques were consistent to the ones described for set \#5 above. The microcosm bottles were labeled S6A, S6B, S6C (Samples, Set\#6, Bottle A, B, \& C), C6A, C6B,C6C (Controls, Set \#6, Bottle A, B, \& C), K6A, K6B, K6C (Killed Control, Set \#6, Bottle A, B, \& C) and stored in the dark at $25^{\circ} \mathrm{C}$, under quiescent conditions, with the liquid in contact with the septa. 
Results of microcosm study set \#6 TCE analysis are presented in Table 4.

Table 4. Results of Microcosm Study Set \#6

\begin{tabular}{|c|c|c|c|c|}
\hline Sample & Day & $\begin{array}{l}\text { TCE } \\
\text { (ppb) }\end{array}$ & $\begin{array}{l}\text { Gas Produced } \\
(\mathrm{ml})\end{array}$ & $\begin{array}{c}\text { Methane Composition } \\
(\%)\end{array}$ \\
\hline$C 5_{A B C}$ & $\overline{0}$ & 1001 & & \\
\hline $\mathrm{KS}_{\mathrm{ABC}}$ & " & 1099 & & \\
\hline $55_{\mathrm{ABC}}$ & $\pi$ & 1001 & - & - \\
\hline & & & & \\
\hline$C 5_{\mathrm{ABC}}$ & 2 & 956 & & \\
\hline$\overline{\mathrm{K}} 5_{\mathrm{ABC}}$ & $\pi$ & 1043 & & \\
\hline $\mathbf{S 5 _ { \mathrm { ABC } }}$ & " & 904 & 21 & 23 \\
\hline$C 5_{A B C}$ & 4 & 1013 & & \\
\hline$\overline{\mathrm{K}} 5_{\mathrm{ABC}}$ & 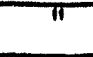 & 1057 & & \\
\hline $55_{\mathrm{ABC}}$ & " & 920 & 16 & - \\
\hline & & & & \\
\hline$C 5_{A B C}$ & 7 & 1041 & & \\
\hline $\mathrm{K} 5_{\mathrm{ABC}}$ & & 1062 & & \\
\hline $55_{A B C}$ & $\pi$ & 949 & - & - \\
\hline
\end{tabular}

C5 $5_{\mathrm{ABC}}$ - Average of Microcosm Water Control Bottles A, B, \& C

$\mathrm{KS} \mathrm{ABC}_{\mathrm{ABC}}$ - Average of Microcosm Killed Control Bottles A, B, \& C

S5 ${ }_{\mathrm{ABC}}$ - Average of Microcosm Active Sample Bottles A, B, \& C

Microcosm study set \#6 results are similar to the relative trend experienced in set \#5 data. The initial TCE concentrations were higher than expected. The bottles were prepared with the same total mass of TCE as in the fifth set and the expected TCE liquid concentration was expected to be around $886 \mathrm{ppb}$. The consistently high results may be due to an error during microcosm preparation, or associated with previously described GC sensitivity drift. Standards were checked at the time of initial analysis and results were only found to be slightly high as compared with previous standardization. The standard curve was not corrected at this time due to time constraints (standardization requires a full day of analysis) and the results were expected to be slightly higher. Despite the higher than expected initial concentrations, the significant finding during set \#6 analysis is the relative decrease in active sample TCE concentration seen as the experiment proceeded. Active samples were found to be producing gas and early results indicate significant methane production. The sixth set of microcosm bottles continue to be maintained on a two day cycle.

5.2.5 Conclusions Installation of a new purge and trap and an ELCD detector has provided a workable analytical system for TCE measurement. While TCE identification and quantification 
seem to be reliable during standardization, the sensitivity drift that has been experienced during microcosm studies sets \#5 \& \#6 make it difficult to interpret data with respect to time. However, in a relative sense the general trend experienced during microcosm studies \#5 \& \#6 support successful anaerobic biological activity and the reductive dehalogenation process is expected to become evident during fitture microcosm analysis.

\subsection{Research Planned for Next Quarter}

\subsubsection{TCE Degradation Rate Evaluation}

The aerobic degradation rate experiments will be continued into the next quarter at initial TCE concentration levels of $2000 \mathrm{ppb}$. Methane will be added only once immediately before the addition of TCE and transfer to the Engineering Labs for analysis. Once degradation rates using methane as the primary substrate have been established, we will use similar experiments to determine TCE degradation rates using other primary substrates or carbon sources such as formate and methanol in addition to methane. The effects of the addition of hydrogen peroxide verses or in addition to oxygen as a source of reducing power will also be evaluated using data previcusly acquired that showed levels of hydrogen peroxide as high as $2600 \mathrm{ppm}$ were non toxic to cultures of $M$. capsulatus. Because the analysis time required for the SRI gas chromatograph in the Biochemistry Lab will be much shorter than that required for the purge and trap gas chromatograph now in use (approximately 5 verses 30 minutes), once the stand-up detector previously mentioned is in place, analysis and degradation rate determinations can proceed much more quickly. Access to a gas chromatographic unit in the Biochemistry Lab would also provide a practical means for accurate determination of TCE toxicity on established cultures of viable bacteria, allowing for immediate and continuous determination of actual TCE concentration in culture media under growth conditions.

Establishing aerobic degradation and transformation rates of TCE into its daughter products or to complete mineralization is a critical step in this research project. Once the optimum provisions of primary substrates and reducing power have been established, we will begin to examine the effect of these optimimum compounds and concentrations on the actual degradation of TCE that may be found under field conditions. In the subsurface it is likely that oxygen and methane will be rapidly depleted, and introduction of these substrates will be a difficult engineering problem due to their low mobility. However, substances such as methanol and formate can provide energy to the organism for TCE oxidation (Henry and Grbic-Galic, 1991), and are likely to have significantly greater mobility in the subsurface than methane. In addition, hydrogen peroxide can be converted to oxygen in the subsurface, and is also likely to have greater mobility than $\mathrm{O} 2$. This aspect of research will be performed using glass columns containing soil, TCE, and methanotrophic bacteria. The columns will be flushed either continuously or periodically with a carbon source and an oxygen source, and samples will be taken periodically and analyzed or TCE and bacterial growth or subsidence. A control column flushed with nitrogen gas will also be examined to insure that the gas is not volatilizing the TCE. In this way, the relationship between the rate of growth and rate of TCE degradation as a function of substrate and oxygen 
addition under field-simulated conditions may be determined.

5.3.2 Evaluation of Reductive Dehalogenation Using Serum Bottle Microcosms Preliminary microcosm studies have demonstrated the ability of anaerobic cultures developed in the W.V.U. Civil/Environmental laboratories to reductively dehalogenate trichloroethylene. However, several follow-on microcosms studies are planned for the next quarter. Initially, the problem of sensitivity drift with GC analyses must be resolved. In addition, samples will continue to be taken from microcosm set\#6 over time to follow the progression of TCE biodegradation.

A number of other factors, not considered during preliminary microcosm studies, will be investigated during future microcosm experiments. Measurements of total suspended solids and volatile suspended will be taken in order to establish biological transformation rates, and to help determine biomass sorption coefficients. As noted from the preiminary studies, contaminant partitioning is an important concern that must be addressed in order to evaluate biodegradation. During future microcosm studies VOC partitioning will be evaluated and contaminant total mass will be calculated using the following relationships:

$$
\begin{aligned}
& \mathrm{M}_{\mathrm{T}}=\mathrm{H}^{* *} \mathrm{C}_{1}{ }^{*} \mathrm{~V}_{\mathrm{g}}+\mathrm{C}_{1}^{*} \mathrm{~V}_{1}+\mathrm{K}_{\mathrm{p}}^{*} \mathrm{X}^{*} \mathrm{C}_{1}{ }^{*} \mathrm{~V}_{1} \\
& \text { "Total "Mass in "Mass in "Mass on } \\
& \text { Mass" } \text { Gas" } \quad \text { Liquid" Solids" }
\end{aligned}
$$

Where:

$$
\begin{aligned}
& \mathbf{M}_{\mathrm{T}}=\text { Total Mass; ug } \\
& \mathbf{H}^{\prime}=\text { Dimensionless Henry's Constant } \\
& \mathbf{C}_{1}=\text { Concentration in the Liquid Phase; ppb } \\
& \mathbf{V}_{\mathbf{g}}=\text { Volume of Headspace Gas; } 1 \\
& \mathbf{V}_{1}=\text { Volume of Liquid; } 1 \\
& \mathbf{K}_{\mathbf{p}}=\text { Sorption Coefficient; } 1 / \mathrm{gr} \text { VSS } \\
& \mathbf{X}=\text { Concentration of Biomass; } \mathrm{gr} \text { VSS } / 1
\end{aligned}
$$

To use the above relationship Henry's constants (H'), biomass concentrations (X), and sorption coefficients $\left(\mathrm{K}_{\mathrm{p}}\right)$, must be known or found experimentally. Previous researchers (Long, et al.,1993; Ashworth, et al., 1988; \& McKay and Shiu, 1981) have determined Henry's constants for the VOCs of interest and these values will be used to evaluate the partitioning between the liquid and gas phase. As previously stated, measurements will be taken to establish biomass concentration in the microcosms and these results will serve as the biomass concentration (X) parameter in the above relationship. In order to determine sorption coefficients $\left(\mathrm{K}_{\mathrm{p}}\right)$ killed culture controls will be prepared and evaluated during future microcosm studies. The procedure for assessing sorption of VOCs to biological solids requires that the bacterial cultures not be metabolically active. Killed culture controls will be prepared in triplicate by removing mixed liquor samples from the maintenance reactor, placing the liquor into the serum bottles, and autoclaving the bottles for 30 minutes at $250^{\circ} \mathrm{C}$. Once cooled, the bottles will be spiked with mercuric chloride to prevent biological activity. Finally, the killed culture controls will be spiked 
with contaminant (PCE, TCE, etc...) at the same concentrations as the inoculated samples and distilled water controls, and all bottles will be stored as previously described. During the time of the microcosm study liquid samples will be analyzed from the three sets of serum bottles, and using the mass balance relationship VOC partitioning will be evaluated.

5.3.3 Design and Operation of Columns for VOC Biotransformation- Engineering and Biochemistry As noted in the last quarterly report (October 1993, Section 5.5.3), the phase 3 soil column studies will use upflow glass chromatography columns. The groups in Engineering and Biochemistry will utilize the same type of columns. The Engineering columns will be initially operated anaerobically while the columns in Biochemistry will be aerobic. after initial data is generated, a combined anaerobic-aerobic system will be operated.

It was planned that the anaerobic columns and associated pumps would be purchased, setup and operated last quarter. However, as already noted, no project funds were available for purchase resulting in delay of the work. In November, funds were made available from non-DOE sources to procure one column which was received in December. The column will be sent to the glassblower for addition of ports for recirculation and sample collection as soon as project funds are available and pumps will also be ordered. It is anticipated that at least one anaerobic column can be set up and initial data generated during the next quarter depending on equipment procurement. It is also planned to procure and setup an aerobic column system this quarter.

The chromatography columns (Figure 1) will be $5.0 \mathrm{~cm}$ in diameter and $30.5 \mathrm{~cm}$ long. The column will have an empty bed volume of $600 \mathrm{~cm}^{3}$ and will be operated at a flowrate ranging from $6.25 \mathrm{ml} / \mathrm{hr}$ to $25 \mathrm{ml} / \mathrm{hr}$ (1 to 4 day detention time). A peristaltic pump will be used to deliver groundwater flow, while a syringe pump will introduce the contaminants of interest (PCE, TCE,etc..) at a ratio of $1: 5$ or $1: 10 \mathrm{v} / \mathrm{v}$.

Sand bed media ( $\sim 3 \mathrm{~mm}$ dia.) will be inoculated with maintenance reactor liquid to establish an anaerobic soil culture for reductive dehalogenation studies, while methanotrophs from Shiemke's lab will be used to develop aerobic column studies. The columns will be continuously percolated in an upflow mode under saturated conditions with groundwater amended with an anaerobic or aerobic mineral media, plus electron donors (methanol \& acetate) for anaerobic test or electron acceptors (methane) and an oxygen source (hydrogen peroxide) during aerobic testing. Ultimately the testing will involve a sequential treatment train and columns will be operated accordingly.

Side ports used for sampling purposes will be fitted with a miniature inert 2-way valve with teflon connections and luer-lock fittings. A $5 \mathrm{ml}$ gastight luer-lock syringe will be used to withdraw samples from the column during operation. Sampling design was selected in order to minimize flow interruption and avoid unnecessary loss of volatiles. All connections, fittings and tubing will be made of teflon in order to minimize contaminant adsorption. During column operation necessary measures will be taken in order to maintain ambient temperatures and a dark environment. 


\subsection{REFERENCES}

Ashworth, R.A. (1988) "Air-water partitioning coefficients of organics in dilute aqueous solutions", J. Hazardous Mater., Vol. 18, No. 1, pp 25-36.

Anthony, C. (1986) "Bacterial Oxidation of Methane and Methanol" Adv. Microb. Physiol., Vol. 27, pp 113-209.

Brusseau, G. A., H.-C. Tsien, R. S. Hanson, and L. P. Wackett (1990) "Optimization of Trichloroethylene Oxidation by Methanotrophs and the Use of a Colorimetric Assay to Detect Soluble Methane Monooxygenase Activity", Biodegradation, Vol. 1, pp. 19-29.

DiSpirito, A. A., J. Gulledge, A. K. Shiemke, J. C. Murrell, M. E. Lidstrom, and C. L. Krema (1992) "Trichloroethylene Oxidation by the Membrane Associated Methane Monooxygenase in Type I, Type II, and Type X Methanotrophs", Biodegradation, Vol. 2, pp 151-164.

Dalton, H. and D.E. Stirling (1982) "Cometabolism", Philos. Trans. R. Soc. Lond., 297, pp 481496.

Freedman, D.L. and J.M. Gossett (1989) " Biological Reductive Dechlorination of Tetrachlorethylene and Trichlorethylene to Ethylene Under Methanogenic Conditions", Appl. and Env. Microbiol., pp 2144-2151.

Henry, S. and D. Grbic-Galic (1991) "Influence of Endogenous and Exogenous Electron Donors and Trichloroethylene Oxidation Toxicity on Trichloroethylene Oxidation by Methanotrophic Cultures from a Groundwater Aquifer", Appl. Environ. Microbiol., Vol. 55, pp 244-263.

Henson, J.M. et al. (1988) "Microbial Removal of Halogenated Methanes, Ethanes and Ethylene in an Aerobic Soil Exposed to Methane", FEMS Microbiology Ecology, 53 (3/4), pp 193-201.

Kaster, M. (1991) "Reductive Dechlorination of Tri- and Tetrachloroethylenes Depends on Transititon from Aerobic to Anaerobic Conditions", Appl. and Environ. Microbiology, Vol 57, No. 7, pp. 2039-46.

Little, C. D., A. V. Palumbo, S. E. Herbes, M. E. Lidstrom, R. L. Tyndall, and P. J. Gilmer (1988) "Trichloroethylene Biodegradation by a Methane-Oxidizing Bacterium" Appl. Environ. Microbiol., Vol. 54, pp 951-956.

Long, J.L. et al. (1993) "Anaerobic and Aerobic Treatment of Chlorinated Aliphatic Compounds", Journal of Environmental Engineering, Vol. 119, No. 2, pp 300-320.

McKay, D. and Shiu, W.Y. (1981) "Critical review of Henry's Law constants for chemicals of environmental interest", J. Physical and Chemical Reference Data, Vol. 10, No. 4, pp 1175-1199. 
Oldenhuis, R, J. M. Ruud, D. B Janssen, and B Witholt (1989) "Degradation of Chlorinated Aliphatic Hydrocarbons by Methylosinus trichosporium OB3b Expressing Soluble Methane Monooxygenase", Applied and Environmental Microbiology, Vol 55, No. 11, pp 2819-2826.

Reiser-Roberts, E. 1992 Bioremediation of Petroleum Contaminated Sites, C. K. Smoley, Ed., CRC Press, Bocaraton,FL.

Sims, J.L., R.C. Sims, and J.E. Mathews (1990) "Approach to Bioremediation of Contaminated Soil", Hazardous Waste and Hazardous Materials, V. 7, pp 117-149. 
7.0 Appendix 1

GAS CHROMATOGRAPHY TROUBLESHOOTING HISTORY

June 7 - Pat Diehl (Hewlett Packard)

- Roger Frame (Miles Corporation)

- Install Tekmar purge and trap unit (LS 2 - Miles donation) and Hewlett Packard GC with electron capture detector

June 15 - Pat Diehl test (via telephone) test to isolate problem order new traps

June 28 - condition and install new trap

June 29 -Tekmar suggestion (via telephone) solvent flush

June 30 - order solvent flush kit

July 9 - flush entire purge and trap unit through to GCwith methanol and water as per instructions. Discover leak

July - Roger Frame

-fix leak

-run Tekmar leak test (via telephone)-no additional leaks found

July -Pat Diehl

ran HP standard with direct injection, G.C. okay

Aug. 2 - Install and condition new used column donated by Miles

Aug. $\quad$ - Order new demo purge and trap from Tekmar

Oct. 14 - Roger Frame

- install new purge and trap (LSC 2000) with moisture control module

Oct. 15 - Run blank samples, still getting baseline drift, but best baseline obtained to date

Oct. 17 - Install and condition Poraplot $Q$ column, with intention of developing a TCE standard curve.

- Baseline erratic

Oct. 18 - Switch column back to J\&W DB-624 and check for leaks.

- Fax information to Lou Fox

Oct. - Continue running blanks

- Check background signal, high signal values observed

- Change graphite ferrules on column and recondition

Oct. 27 - Check background signal, better results which are in normal range

- Run blanks and analyze 0.2 ppb TCE

- Still see "Iump", but at attenuation of 7 we get relative flat baseline and TCE Spike.

Oct. 29 - Attempt to analyze 0.1 and 0.3 ppb TCE multiple times, results not consistent or reproducible.

Nov. - Attempt to improve analysis by increasing sample volume, results still not consistent. 
TO: Kevin Mckavney and Lou Fox

Hewlett Packard

FROM: Bill Sack, Kevin Jones and Joan Cuddeback

West Virginia University

SUBJECT:Facts and Findings Regarding GC Analysis ofHalogenated VOCs

Problems associated with our attempts to acquire a workable VOC analysis system continue to exist. The HP 5890 Series II GC with Tekmar LSC 2000 purge and trap concentrator (with moisture control module) and an electron capture detector (ECD) has not provided us with satisfactory results, which in our opinion would allow reliable groundwater VOC sample analysis. Since installation, numerous alterations and adjustments have been made via technical support from HP, Tekmar, and Miles Inc. in the hopes of obtaining a flat (useable) baseline response for blank organic-free water.

Problems have persisted through all attempts and parameter changes, and at best we have obtained a relatively noise-free baseline with a noisy "lump" surrounding the retention time of TCE. This "lump", in particular has remained consistently through all of the nearly $\mathbf{3 0 0}$ runs to date and according to Dr. Snider of PKC Environmental (a local environmental lab), is probably due to water vapor. The "lump" is not evident at an attenuation of 7 (Maximum obtainable attenuation $=10$ ) and the suggested (by Lou Fox) concentration of $0.2 \mathrm{ppb}$ of TCE does result in defined peak, however results have not been consistent or reproducible.

We have become very wary of and frustrated with our analytical ECD-purge and trap combined system. Our analytical needs are simple (aqueous samples of PCE, TCE, DCE and possibly VC). We have reiterated our needs to Lou Fox and Kevin Mckanney of Hewlett Packard in hopes they will suggest a system more suitable to the purposes of our research. In brief, our concerns with using an ECD for our analytical purposes are as follows:

1. Vinyl Chloride detection

- "The inherent insensitivity of the ECD to many monochlorinated compounds......" (HP Book -"Detectors for Gas Chromatography", p. 42) - "even with the Poraplot Q column, VC may not be detectible"

(Lou Fox)

2. EPA Method 601/602

- Specifies analysis of volatiles by purge and trap in combination with ELCD

- ECD not recommended, P. Sandria (Book - "High Resolution Gas

Chromatography") 
3. We are looking for a minimum detection level of $5 \mathrm{ppb}$ for PCE, TCE, DCE and $2 \mathrm{ppb}$ for $\mathrm{VC}$,

- at $0.2 \mathrm{ppb}$, the ECD in combination with purge and trap is requiring an attenuation of 7 for integration and the maximum allowable is an attenuation of 10 .

- With this excessive kind of sensitivity and our inexpertise, how much accuracy are we sacrificing with all the sample dilutions that will be necessary to get within standard curve range, let alone making a reliable standard curve.

4. Trap concerns (to get rid of water)

- Companies suggest, even with the moisture control module, that different traps may be necessary to avoid the water "lump", EPA Method 601 specific the trap that we are currently using, will a different trap sacrifice VOC (PCE, TCE, DCE, VC) selectivity.

\section{Safety Concerns}

- ECD should be vented into a hood due to radioactivity according to HP manuals. This did not come up in installation.

- ECD must be "wipe tested" every six months, has this been scheduled and what type of costs are involved

6. We are using the purge and trap unit to avoid extractions of volatile compounds. According to OI, sample clean-up is required when using an ECD since it is non-selective and so sensitive.

7. Methanol and methane are not desired due to causing background peaks with an ECD (Tekmar letter), both of the will be present in our samples.

Conversations with representatives of a number of companies have led us to believe that the use of an electron capture detector with a purge and trap unit is conducive to problems.

U.S. Gelogical Survey (Ted Ehlke)

- Never heard of the ECD/P\&T combination

- "may see excessive baseline noise and never get a clean baseline"

Suplcico (Bob Shirey, Technical Support)

- ECD sensitive to pressure variation which results from P\&T analysis

- Monochlorinated compounds will be a problem for an ECD

- ECD has a limited linear range

- ECD is very sensitve to water

- "there's no choice, ELCD is what you want" 
Restek (Rick Crago, Technical Support)

- ECD is very touchy to water and small leaks

- Does not know anyone using ECD/P\&T

- May be able to use extraction and direct inject

- Trick for water vapor problem may be the trap packing material (suggest

Volcarb 4000 trap), must also be concerned with purging and desorbing efficiency.

- Integration will be effected by water vapor effects on baseline

HP (Lou Fox)

- Can't get vinyl chloride

- Saturate or overload detector if low concentrations are exceeded

(i.e approx. 1 to $2 \mathrm{ppb}$ )

HP (Catalogue Chromatograms)

- Purge and Trap analysis only appear with an ELCD

(ECD always shown with headspace or extraction methods)

OI Corp. (Mary Beth)

- Sample will have to be cleaned-up or extracted with an ECD

- "If you mentioned using direct samples in a purge and trap, HP should have

known to use an ELCD"

EPA Methods (601 \& 502.1)

- Discuss purge and trap and volatiles in combination with an ELCD or microcoulmetric detectors, and alternatives may be used but have to be reproducible.

- "An electrolytic conductivity or microcoulmetric detector is required. These halogen-specific systems eliminate misidentification due to non-organohalides which are co-extracted during the purge step."

SRI

- "Because the ELCD detector is specific for halogenated compounds, it is not susceptible to interference from water which may be unintentionally injected into the system when performing purge and trap analysis, nor will it respond to non-halogens at all"

Ultra Scientific (John Yanusas)

- Best detector system would be the PID/Hall, go after double bonds and chlorinated compounds.

- Problems will be encountered if you try to work at the extremely low (ppt) concentration, you will never know if your really seeing whats in your sample.

- Only way to work with the ECD would be to do extrations, follow EPA Method 504, but this is alot of extra work. 


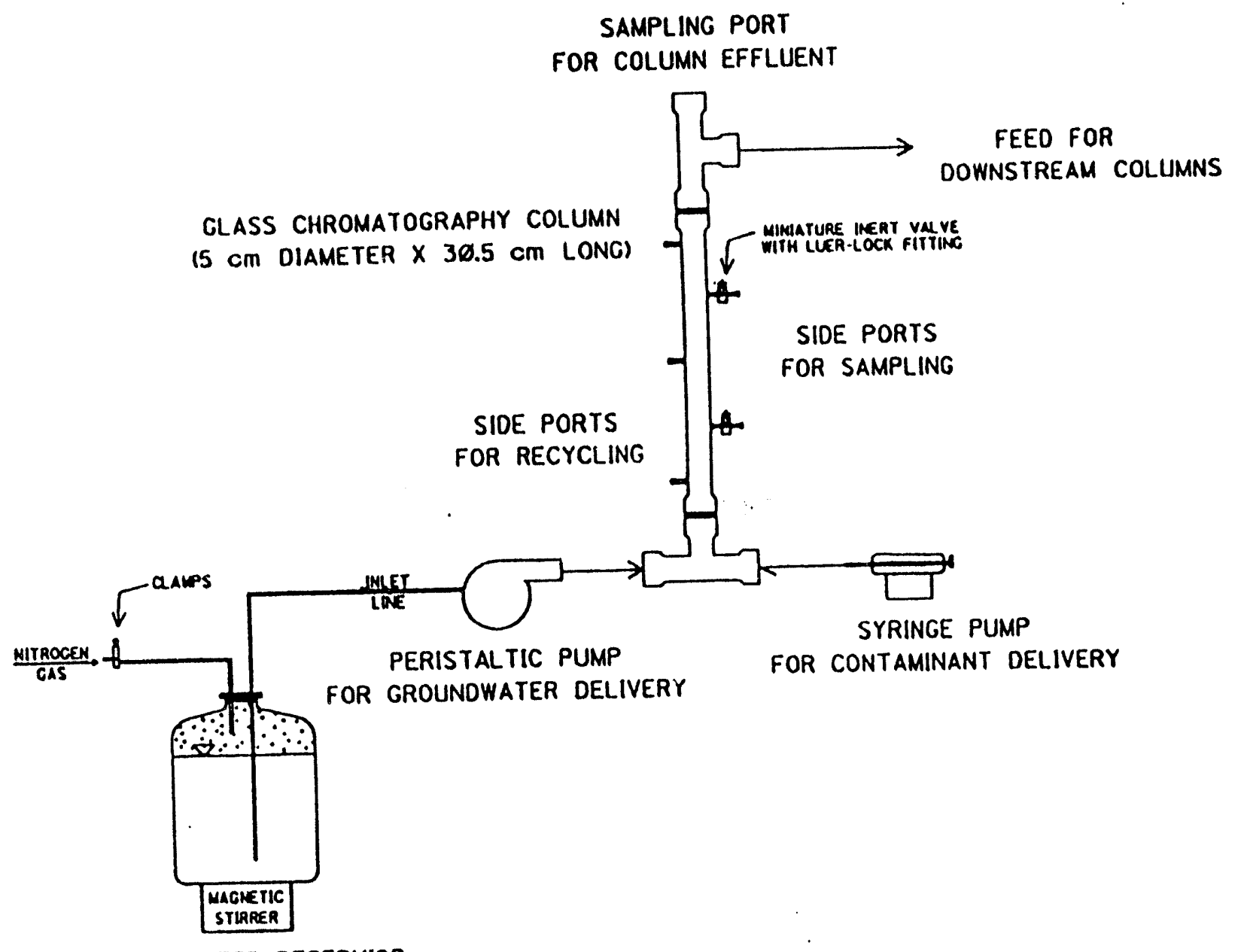

GROUNOWATER RESERVIOR

FIGURE I. SOIL COLUMNS 
Excavation Systems for Hazardous Waste Sites: Dust Control Methods for Insitu Nuclear Waste Handling

(Project MC-4)

\author{
Quarterly Report for Period \\ for Period October 1 through December 31, 1993
}

Work Performed Under Contract

No.: DE-FC21-92MC29467

\author{
For \\ U.S. Department of Energy \\ Office of Fossil Energy \\ Morgantown Energy Technology Center \\ Morgantown, West Virginia
}

By

Syd S. Peng, Department of Mining Engineering

Felicia F. Peng, Department of Mineral Processing Engineering

West Virginia University

Morgantown, West Virginia 


\begin{abstract}
Literature review on the remediation of hazardous wastes, especially for dust/vapor control on in-situ hazardous waste handling has been done last year. There are some commercially available dust/vapor technologies developed by DOE, EPA, civil and mining industries. But it is still questionable whether or not these technologies can be used for clean up of nuclear waste sites since the characteristics and deposit of nuclear wastes, are somewhat different from those of hazardous waste sites.

To look for effective technologies for dust/vapor control on in-situ nuclear waste handling, it is necessary to know an outline of the nuclear wastes with its form, disposal and current treatment technologies. These factors also greatly contribute to the proper selection and effectiveness of dust/vapor control methods except waste material handling.

This quarterly report (October 1 through December 31, 1993) briefly describes an overview of the nuclear wastes and its disposal, introduces some innovative technologies for the treatment of nuclear wastes and discusses potential technologies of dust/vapor control due to in-situ nuclear waste handling.
\end{abstract}


ABSTRACT $\ldots \ldots \ldots \ldots \ldots \ldots \ldots \ldots \ldots \ldots$ ii

TABLE OF CONTENTS $\ldots \ldots \ldots \ldots \ldots \ldots \ldots$ iii

LIST OF FIGURES $\ldots \ldots \ldots \ldots \ldots \ldots \ldots \ldots \ldots \ldots$

1 INTRODUCTION $\ldots \ldots \ldots \ldots \ldots \ldots \ldots \ldots \ldots$

2 OVERVIEW OF NUCLEAR WASTE AND ITS DISPOSAL $\ldots \ldots \ldots \ldots 2$

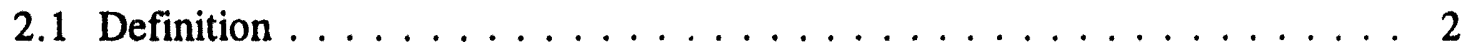

2.2 Current Nuclear Waste Forms $\ldots \ldots \ldots \ldots \ldots \ldots \ldots \ldots$

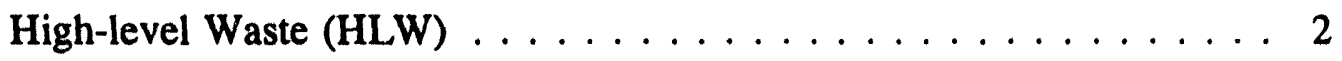

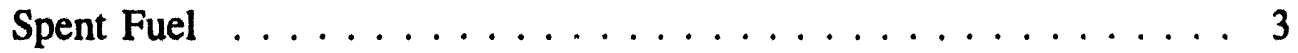

Commercial High-level Waste $\ldots \ldots \ldots \ldots \ldots \ldots \ldots$

Defence High-level Waste . . . . . . . . . . . . . . . 3

Transuanic Waste (TRU) $\ldots \ldots \ldots \ldots \ldots \ldots \ldots \ldots$

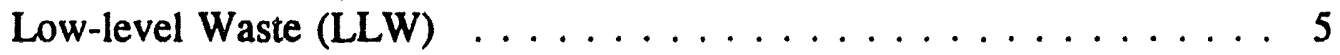

Nuclear Power Reactors . . . . . . . . . . . . . . . 5

Institutions $\ldots \ldots \ldots \ldots \ldots \ldots$

Industry $\ldots \ldots \ldots \ldots \ldots \ldots \ldots \ldots$

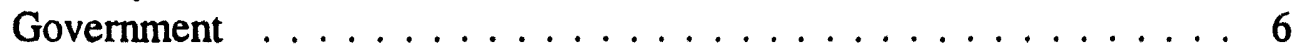

Uranium and Mine Tailings $\ldots \ldots \ldots \ldots \ldots \ldots \ldots$

Phosphypsum Waste .................. 6

2.3 Effect of Nuclear Waste . . . . . . . . . . . . . . 6

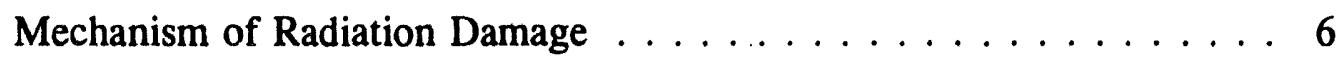

Interaction with Matter $\ldots \ldots \ldots \ldots \ldots \ldots \ldots$

Transport to Human . . . . . . . . . . . . . . 9

Effect on Future Generation . . . . . . . . . . . . . . 9

Damage to Environment $\ldots \ldots \ldots \ldots \ldots \ldots$

2.4 Technology of Nuclear Waste Disposal $\ldots \ldots \ldots \ldots \ldots \ldots \ldots$

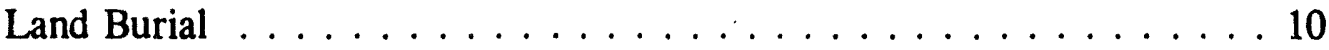

Near-surface Disposal . . . . . . . . . . . . . . . . 10

Intermediate Depth Disposal $\ldots \ldots \ldots \ldots \ldots \ldots$

Abound Mine $\ldots \ldots \ldots \ldots \ldots \ldots \ldots \ldots \ldots \ldots$

Surface Impoundment $\ldots \ldots \ldots \ldots \ldots \ldots \ldots \ldots \ldots$

Geological Repositories . . . . . . . . . . . . . . . 13

Underground Injection Disposal $\ldots \ldots \ldots \ldots \ldots \ldots$

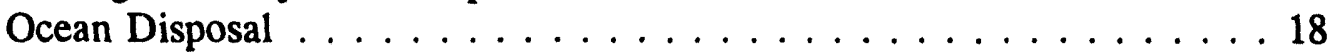


Ocean Disposal of Low-level Waste . . . . . . . . . . . . . . . . 20

Subseabed Disposal . . . . . . . . . . . . . . . . . 20

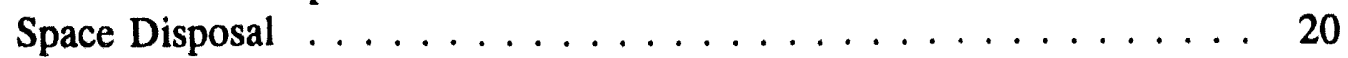

Other Disposals . . . . . . . . . . . . . . . . . 20

3 TECHNOLOGIES WITH POTENTIAL FOR APPLICATION

TO DUST/VAPOR CONTROL AT NUCLEAR WASTE SITES . . . . . . . 20

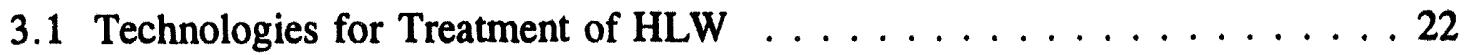

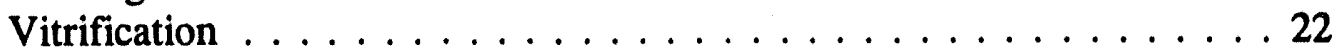

Immobilization/solidification . . . . . . . . . . . . 23

Compact Processing Units (CPUs) $\ldots \ldots \ldots . \ldots \ldots$

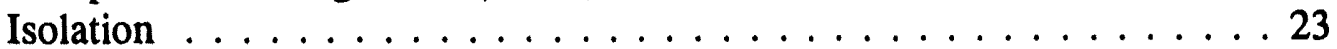

3.2 Technologies for treatment of LLW . . . . . . . . . . . . 24

3.3 Dust/vapor Control in Nuclear Waste Sites . . . . . . . . . . . . . . . 24

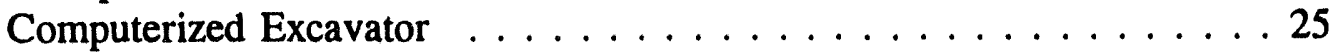

Remote Controlled Excavator . . . . . . . . . . . . . . 25

Pneumatic Excavation System ... . . . . . . . . . . . . 25

Horizontal Walls for Enhanced Access to the Subsurface . . . . . . . . 26

Tele-Operated Remote Control Equipment (TORCE) . . . . . . . . . 26

Hydropermutation System (Hydrop) . . . . . . . . . . . 28

4 SUMMARY AND FUTURE WORK . . . . . . . . . . . . . . . . 29

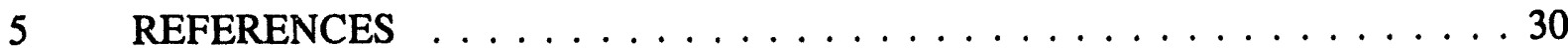


1 The Cycle of Spent Fuel $\ldots \ldots \ldots \ldots \ldots \ldots \ldots \ldots$

2 Predicted Increasing of Nuclear Waste $\ldots \ldots \ldots \ldots \ldots \ldots$

3 Pathway of Nuclear Waste to Environment and Human $\ldots \ldots \ldots \ldots$

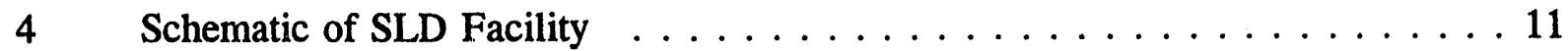

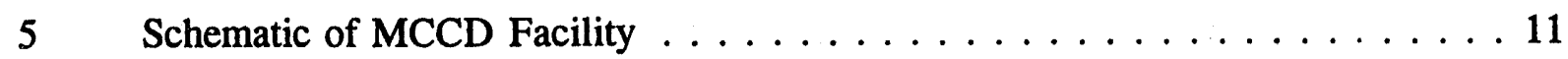

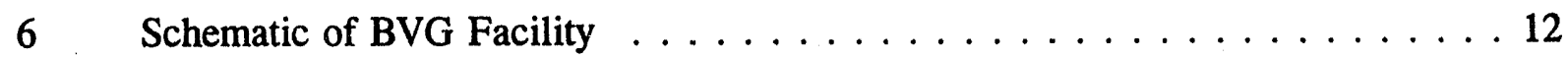

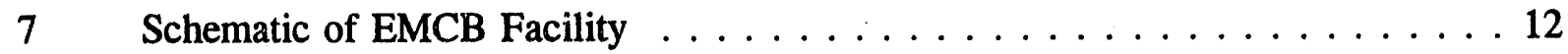

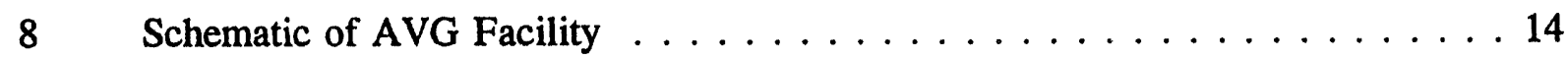

9 Diagram of Intermediate Depth Disposal Using Shaft $\ldots \ldots \ldots \ldots \ldots 15$

10 Schematic Structure of Surface Impoundment Disposal $\ldots \ldots \ldots 16$

11 Schematic Illustration of Geological Repository Disposal . . . . . . . . . 17

12 Location of Candidate Sites for First Geological Repository . . . . . . . . . 19

13 Location of Candidate Sites for Second Geological Repository . . . . . . . . 19

14 Several Proposed Methods for Emplacing Waste in Subseabed Repository . . . . . . . . . . . . . . . . . . 21

15 Orbital Transfer Operations for Space Disposal . . . . . . . . . . . . . 21

16 Layout of Remote Control Station . . . . . . . . . . . . . . . 27 


\section{INTRODUCTION}

The nuclear waste which results from a wide range of processes and applications in using the radioactive materials has been - and currently is being - produced by both government and private sources. Since the radioactive decay within solidified nuclear waste leads on to physical and chemical changes that can alter the rate at which radionucildes are released to the biosphere, the Nuclear Waste Policy Act of 1982 (NWPA) was established by Congress as a comprehensive federal policy for nuclear waste management.

Department of Energy (DOE) is one of the major responsible agencies for developing environmentally safe waste management technologies (commercial and defense waste), and establishing and managing federal radioactive waste repositories. Some innovative technologies for nuclear waste treatment have been demonstrated or are currently being developed. Dust/vapor control for in-situ nuclear waste handling is also emphasized by DOE as one of the major branches for nuclear waste management. Importing or modifying innovative treatment technologies from outside organizations for effective management of nuclear waste is addressed by DOE.

Based on the literature review on dust/vapor control methods for in-situ hazardous waste handling last year, this quarterly report emphasizes nuclear waste management. From the review of nuclear waste forms, its disposal, nuclear waste (HLW and LLW) treatment, and dust/vapor controlling on hazardous waste sites, potential technologies for control nuclear waste dust/vapor are introduced. 


\section{OVERVIEW OF NUCLEAR WASTE AND ITS DISPOSAL}

\subsection{DEFINITION}

Nuclear or radioactive waste is defined as any material that is no longer useful and that contains radioactive isotopes in amounts recognized by the regulatory authorities as posing a potential risk to human health and the environments sufficient to warrant its isolation from the biosphere (Robert. E. B. and C. S. Catherial, 1989). The definition condenses the forms, effects, regulations and disposal of nuclear waste into one sentence. Briefly the contents of the definition are reviewed as follows in terms of the project objective.

\subsection{CURRENT NUCLEAR WASTE FORMS}

Several different schemes have been used to classify nuclear wastes so far. They are based on radioactivity level, state or phases of waste, radiation emission, half-life, retreatment, disposition or potential consequence, etc.. This report adopts a modified radioactivity level based on the radiologic, chemical, and physical characteristics that affect the design of systems for handling, treating and disposal of the nuclear waste. Under the classification of modified radioactivity level, the nuclear waste forms are partitioned into high-level waste (HLW), transuranic waste (TRU), low-level waste (LLW), uranium mine and mill tailings and phosphogypsum waste.

\section{(1) HIGH-LEVEL WASTE (HLW)}

It was administratively broadened by the U.S. Nuclear Regulatory Commission (NRC) in 1981 to include:

(i) irradiated reactor fuel

(ii) liquid wastes resulting from the operation of the first cycle solvent extraction system, or equivalent, and the concentrated wastes from subsequent extraction cycles, or equivalent, in a facility for reprocessing irradiated reactor fuel

(iii) solids into which such liquid wastes have been converted

By this definition, the high-level waste includes those produced from the reprocessing of fuel irradiated in government facilities weapons production, similar wastes produced in commercial reprocessing facilities, and unprocessed spent fuel. However, spent fuel, 
solidified commercial HLW and defense HLW, which are considered as special types of HLW, with their physical, chemical and radiological characteristics as well as the current plans for packaging, are described below:

- Spent Fuel. It is a waste fuel which is removed from the core and stored on-site underwater in a spent fuel storage pool. The cycle of spent fuel is shown in Fig. 1. The fuel contains highly radioactive fission products and the radiation levels require substantial shielding from workers. The spent fuel is also physically hot as a result of the decay of fission products. All on-site operations involving the spent fuel are performed remotely with the fuel remaining underwater to provide both radiation shielding and cooling. The spent fuel assemblies will be sealed in canisters prior to disposal. Canister material and packaging will be so chosen that it enhances the performance of the final repository system.

. Commercial High-level Waste. Approximately 600,000 gallons of liquid high-level radioactive waste were produced by the operation of the commercial reprocessing facility at West Valley, New York (which was shut down for expansion in 1971) from April, 1966 through December 1971. It was stored in the double-shelled tanks underground at the reprocessing site for permanent storage. This waste is in the process of being solidified into glass matrix, encapsulated and prepared for shipment to the federal repository. It is the only commercial HLW presently expected to exist in the United States. No other commercial reprocessing facility has been established so far.

Defence High-level Waste. There are 10 times more defence HLW than commercial HLW. Comparing the defense HLW with the commercial HLW, it has a lower radioisotope concentration than the commercial HLW and correspondingly lower heat output per canister. The similarities among defence HLW, commercial HLW, and spent fuel are more significant than their differences when considering the requirements for handling and disposal of the waste.

\section{(2) TRANSURANIC WASTE (TRU)}

Radiations that is heavier (having more protons) than uranium are called transuranic isotopes (TRU). Most TRU in existence today, however, have been artificially produced by the irradiation of nuclear fuel. It results from the interaction of uranium and thorium with neutrons and subsequent beta decay. TRU includes isotopes that have long-lives and are highly radiotoxic because they decay by emitting high energy alpha particles. TRU may be present in solidified liquid waste as well as incorporated into fuel cladding hulls and other components filters, sledges, and trash. 


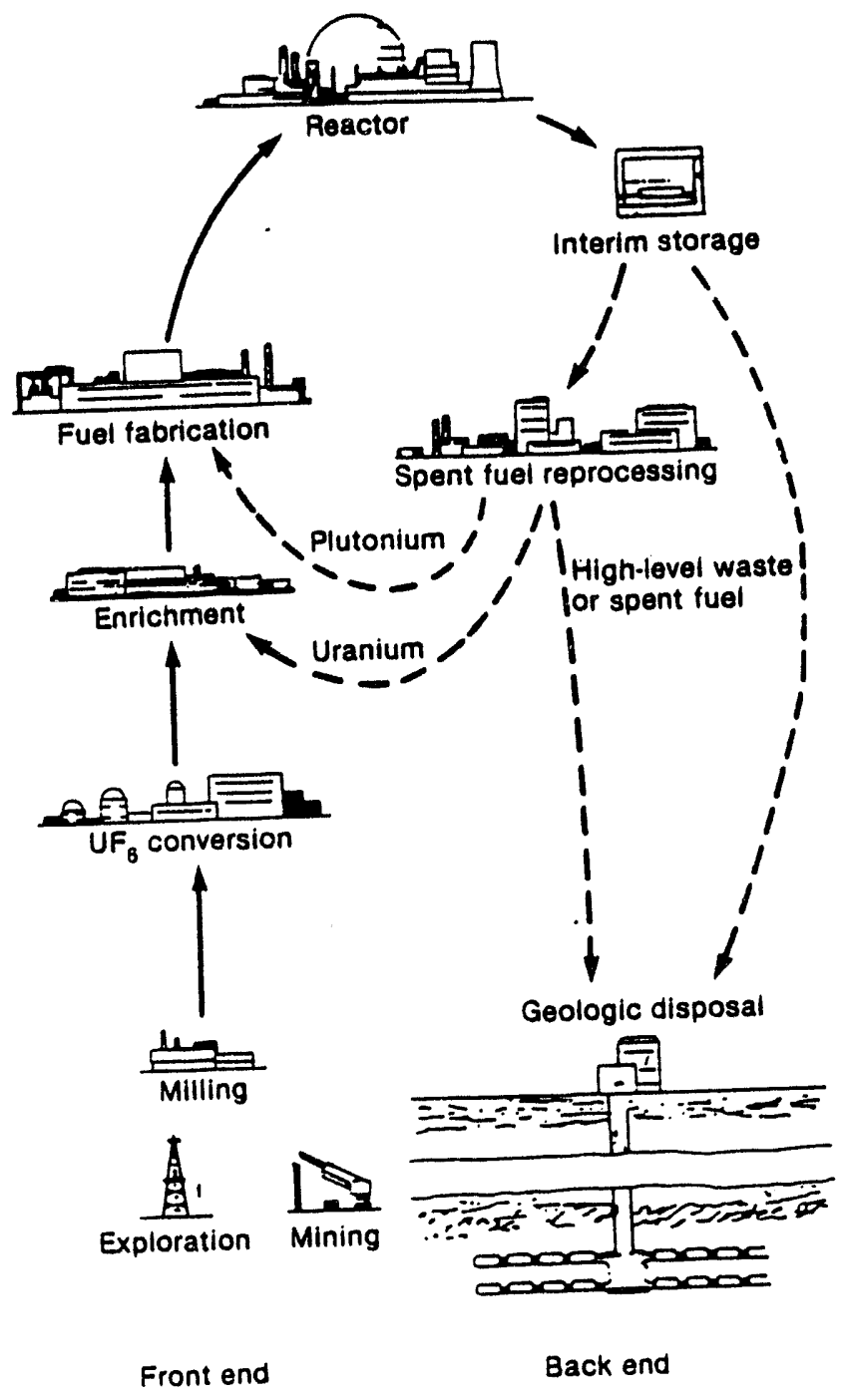

Fig. 1 The Cycle of Spent Fuel (Source: Council on Environment Quality 1981) 


\section{(3) LOW-LEVEL WASTE (LLW)}

LLW is produced - or potentially produced - as a result of any action in which radioactive material is used. LLW characterization is a moving target requiring periodic review and update. This is very different from the HLW the characteristics of which remain relatively stable. The variation in LLW characteristics reflects the very large number of applications, the many individual and independent users, and the development of new applications and packaging and treatment process. The LLW characteristics are described in the following in terms of the major categories of waste generators.

- Nuclear Power Reactors. Fuel is assembled for two major power reactor designs used in the Untied States, pressurized water reactor (PWRs) and boiling water reactors (BWRs). The projected volumes for new power plats were established by NRC for licensing evaluation purposes to be $17,000 \mathrm{ft}^{3}$ for PWRs and 29,000 $\mathrm{ft}^{3}$ for BWRs (NRC 1982). Small amounts of radioactive material are present in the coolant of a power reactor.

. Institutions. Medical and academic institutions use radioactive materials in research, diagnosis and therapy and employ a variety of radioisotopes and material forms to achieve their purposes. The volumes of LLW produced by medical and academic institutions have decreased substantially since 1979 . Much of this, reduction has resulted from the regulatory in that it requires special disposal.

. Industry. Industrial processes that result in the production of LLW include the production and distribution of radioisotopes for materials containing radioisotopes, and the use of radioisotopes for research or testing and in gauges or instrumentation.

- Government. Government operations produced $2 \%$ of the LLW disposal at commercial facilities in 1984 . These wastes are similar to those being shipped by other generator categories. The LLW produced by "atomic energy defense activities" and "federal research and development activities" are not subjected to the provisions of the Low Level Radioactive Waste Policy Act requiring the states to provide disposal capability.

\section{(4) URANIUM MINE AND MINE TAILINGS}

Since uranium is a very concentrated energy source, it is economic to process even the very low concentration ore. Approximately $6 \%$ of the original uranium content is not recovered in milling process such as crushing, grinding, leaching etc. and is retained in the waste. They are a potential large radiation exposure source. The NRC estimated (NRC 1979) that premature deaths due to radon release from 1978 to 2000 from uranium mining and milling operations would be 181 in total. Of this total, $60 \%$ was estimated to be due to 
mining operations.

Active mill tailings sites exist in seven western states in which most of the U. S. uranium production has occurred to date. DOE estimates that 3.48 billion $\mathrm{ft}^{3}$ of commercial tailings had been accumulated through 1982 (DOE 1983). This material contains over 408 thousand $\mathrm{Ci}$ of radioactivity and produces about $9000 \mathrm{~W}$ of thermal power as a result of radioactive decay. Over 5 billion $\mathrm{ft}^{3}$ of tailings will exist by 2000 .

\section{(5) PHOSPHOGYPSUM WASTE}

Phosphate rock contains relative high concentration of uranium. When the phosphate ore is mined, the uranium-bearing material is brought to the earth's surface, crushed, and processed, by screening and flotation. The waste products include slimes and sands similar to uranium mill tailings. The slimes contains about $50 \%$ of the radioactivity in the original ore. An additional $12 \%$ is retained in sand. The phosphogypsum waste represents a large volume of material with relatively high concentration of radionuclides easily accessible to human.

Based on DOE data base (DOE, 1987), the predicted increase of nuclear waste from 1990 throughout 2020 is diagrammed in Fig. 2.

\subsection{EFFECT OF NUCLEAR WASTE}

The effect of nuclear wastes on human is considered as a hazard with time. The principal sources of radiation are alpha decay of the actinide elements and beta decay of the fission products, both of which lead to physical changes (radiation damage) and chemical changes (transmutations). In general, beta decay of the fission products predominates during the first 500 years with high radioactive and high heat generation, with which alpha decay predominates for longer period. The pathway of nuclear waste to environment and eventually to human is analyzed as shown in Fig. 3. The effects of radiation by nuclear waste cover the following aspects:

\section{(1) MECHANISM OF RADIATION DAMAGE}

. Interaction with Matter. The "ionization" and "excitation" are two kinds of energy transfer mechanisms which may cause radiation damage from individual molecules, e.g. carbons, to macromolocules, e.g. proteins and DNA, to cellular effects (membrances andcarbons mycolic cycle), and to major organs. 


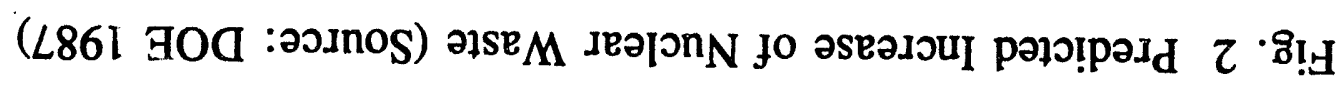
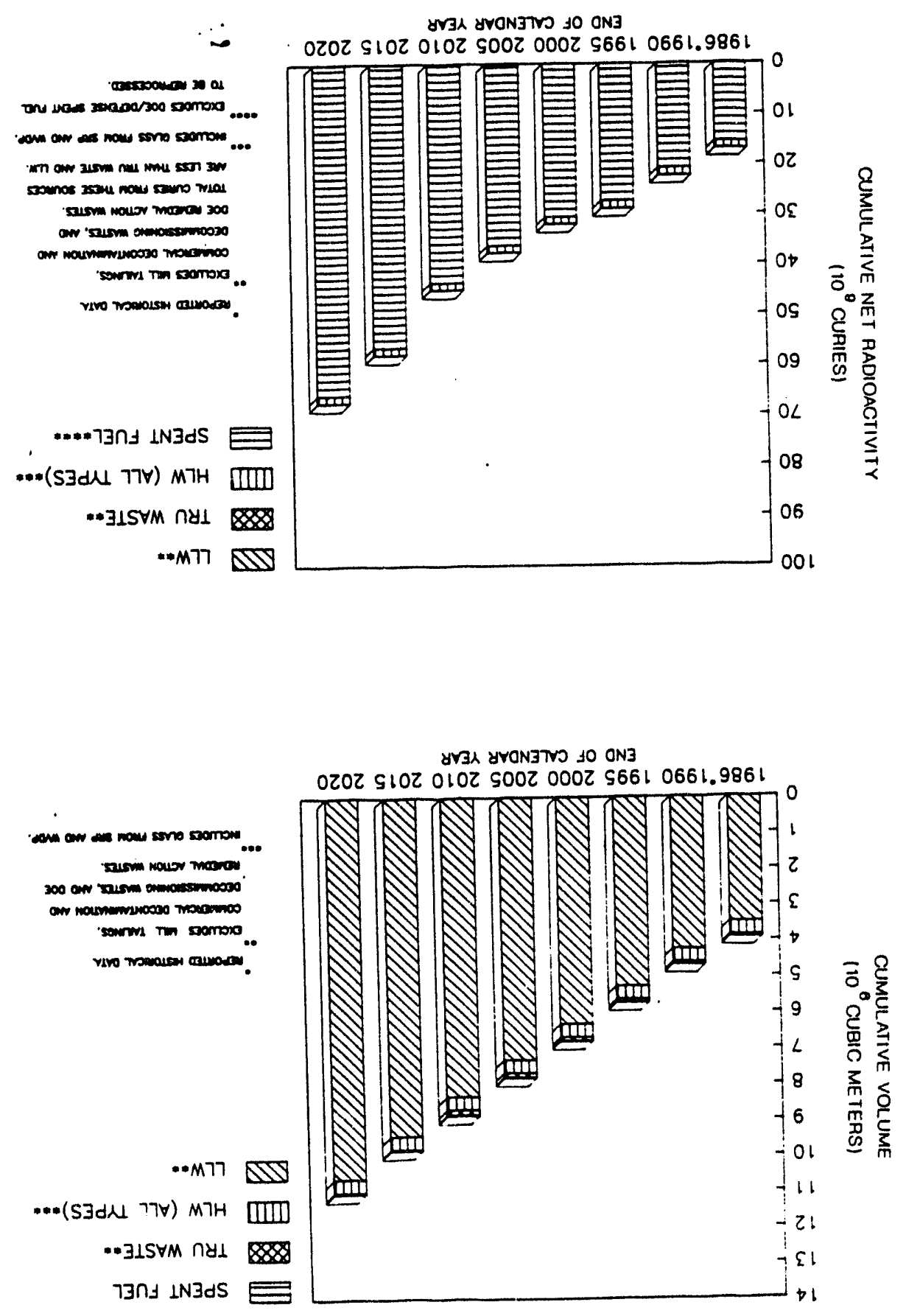


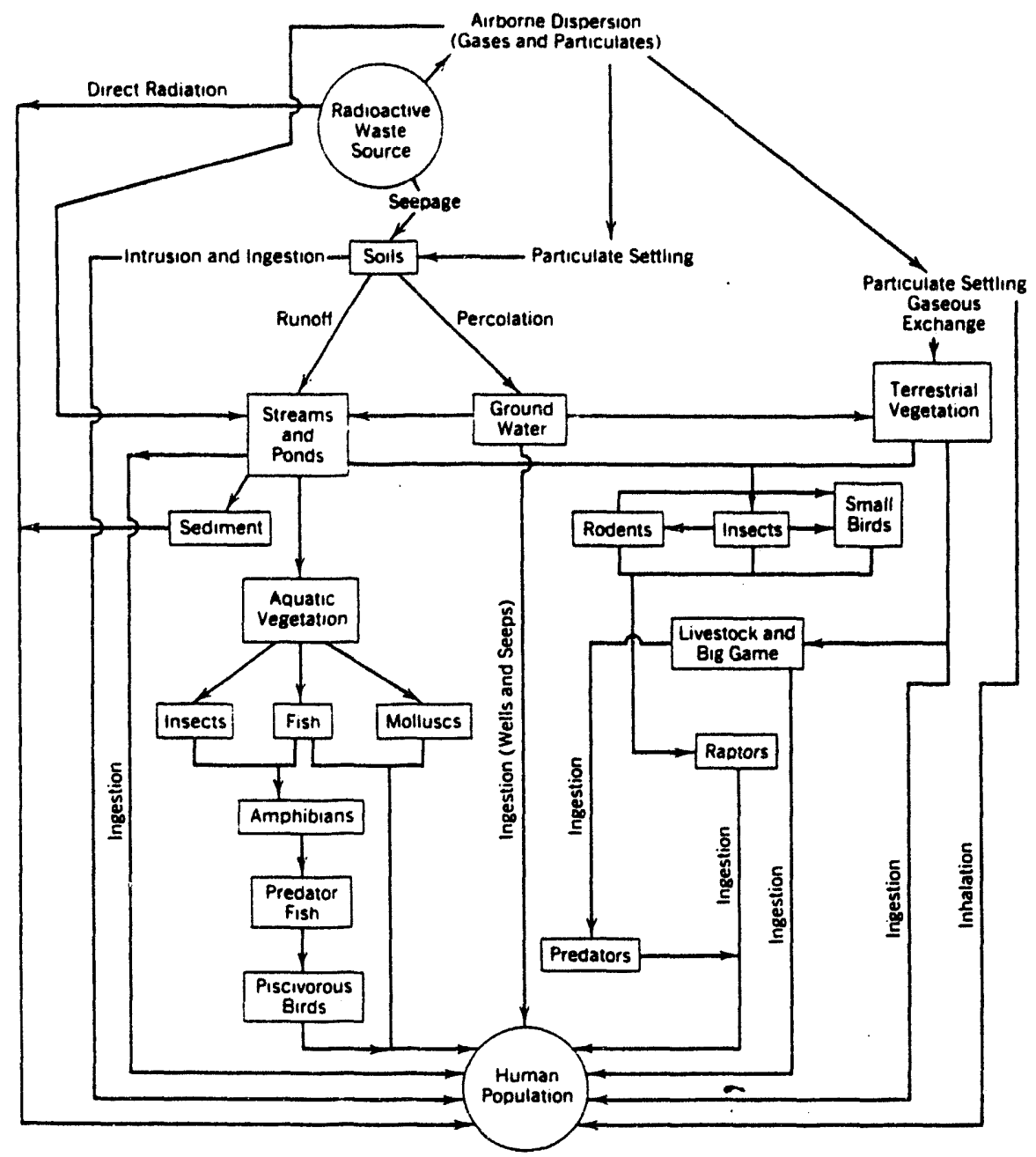

Fig. 3 Pathway of Nuclear Waste to Environment and Human (Source: Rebert and Cartering 1989) 
- Transport to Humans. When radionuclides emit the radiation, they can deliver radiation from outside the body. Alpha and beta particles have to be put inside the body to deliver a dose. Damages to the lung and skill cause by the ingestion of the contaminated water, milk, and other food.

(i) Acute Effects: A dose of 50 rem or more of radiation within minutes to weeks. Effects include increased temperature, malaise and fatigue, nausea and vomiting, and death. Blood changes are the only clinical effects observable from exposures of less $50 \mathrm{rem}$. Blood changes are usually detectable in the $25-50$ rem range and have been detected for exposures as low as 14 rem.

(ii) Somatic Effects: The primary somatic effect from low-level radiation exposure is an increase in frequency of various types of cancer. Cancers caused by low-level radiation delivered at low dose rate are usually indistinguishable from cancers caused by other factors and can be observed only statistically.

\section{(2) EFFECT ON FUTURE GENERATION}

Future effects can result from two separate causes: (1) Generic effects from radiation dose delivered to the present generation; and (2) Somatic effects due to radiation doses received by future generations from exposure to long-lived radionuclides released to the environment by various generations.

\section{(3) DAMAGE TO ENVIRONMENT}

Effects on animals and plants are much the same as on humans. Attitudes towards the environment are complex and vary amongst different individuals, groups and countries but there is a widespread feeling that the environment should not be abused since the quality of the environment is an important factor in determining the quality of human life. Protection of the environment should not be for the sole purpose of benefits the human being and it shuuld also consider the continuing survival of the species as a whole. Careful monitoring may be required to determine what effects are occurring and to ensure that the long-term stability of the ecosystem is not disturbed. 


\subsection{TECHNOLOGY OF NUCLEAR WASTE DISPOSAL}

Under the current regulations, permanent disposal of the high-level waste (HLW), transuranic waste (TRU), low-level waste (LLW), and tailings are required. There are several technologies for nuclear waste disposal which are described according to the nuclear waste forms (DOE, 1987; John, 1985; IAEA, 1981; NRC, 1982 and NRC, 1992).

\section{(1) LAND BURIAL}

Burial in the soil was the earliest method used for disposal of low-level radioactive wastes (LLW). Depending on site conditions related factors such as distance to groundwater and soil sorptive properties, both liquid and solid wastes have been disposed by this method.

There are several variants of and burial technology that have been applied. The choice among them is related to site characteristics, waste characteristics, and costs.

- Near-Surface Disposal. Near-surface disposal is generally defined as emplacement of the waste underneath soil ground about $100 \mathrm{ft}(30 \mathrm{~m})$. Basic requirements are established by NRC in the final Environment Impact Statement on 10 CFR part 61 (NRC 1982). There are several main alternatives that have been proposed to achieve the principles outlined by NRC's regulations.

(i) Shallow-Land Disposal (SLD). SLD is accomplished by constructing trenches that are approximately $30 \mathrm{ft}$ deep as shown in Fig. 4.

(ii) Modular Concrete Canister Disposal (MCCD): MCCD provides an additional engineered barrier between the emplacement waste and the biosphere compared to SLD as shown in Fig. 5. This is achieved by placing the waste into steel-reinforced concrete canisters that are placed in trenches similar to those used for SLD.

(iii) Below Ground Vaults (BGV). BGV disposal, as illustrated in Fig. 6, provides an engineered barrier between the waste packages and the biosphere as well as structure stability by the use of an engineered concrete structure below the nature grade. The structure consists of reinforced concrete walls and floor.

(iv) Earth Mounded Concrete Bunkers (EMCB). EMCB technology, which was originally employed at the waste facility at Centre de la Manche, France, disposes of the waste above grade with an earthen cover. The waste in the tumults is placed in concrete containers similar to those described for MCCD as illustrated in Fig. 7.

(v) Above Ground Vaults (AGV). AVG disposal provides for emplacement of 


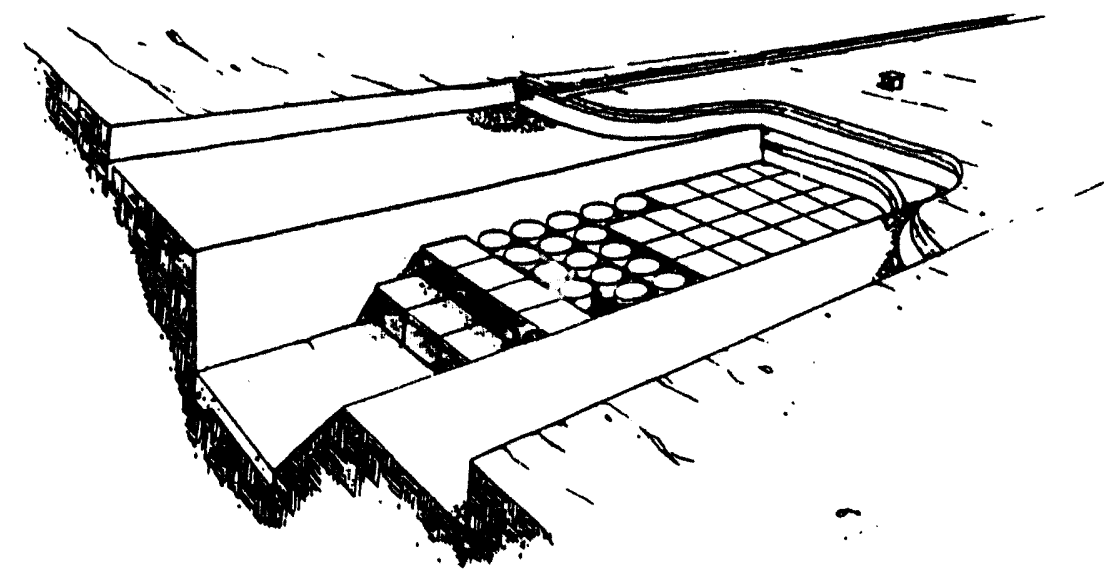

Fig. 4 Schematic of SLD Facility (Source: DOE 1987)

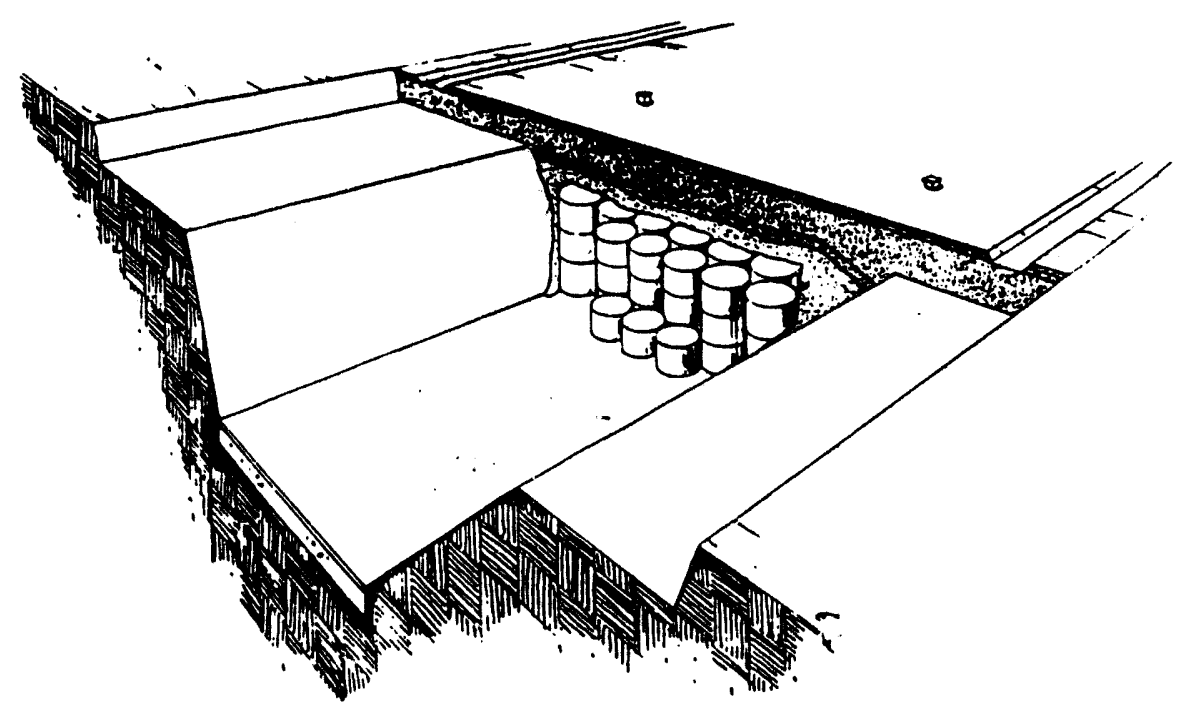

Fig. 5 Schematic of MCCD Facility (Source: DOE 1987) 


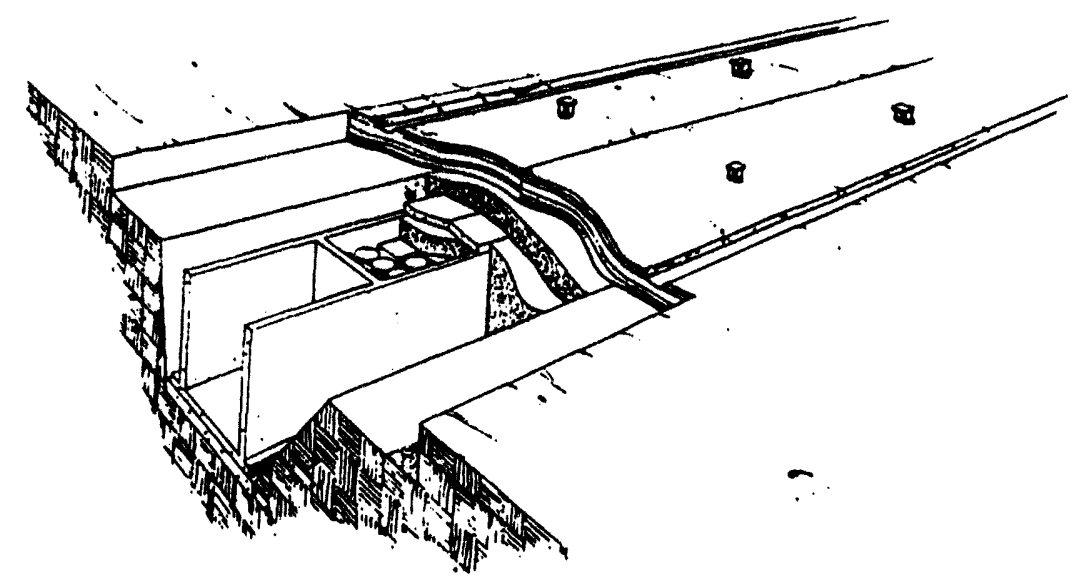

Fig. 6 Schematic of BVG Facility (Source: DOE 1987)

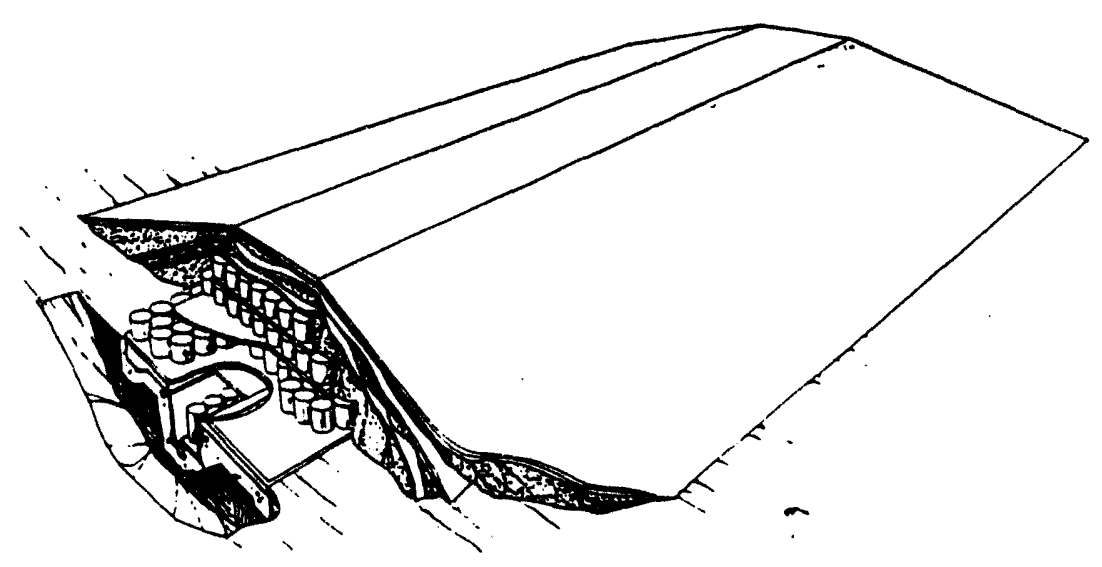

Fig. 7 Schematic of EMCB Facility (Source: DOE 1987) 
waste in reinforced concrete structure above the natural grade. Like BGVs, AGVs are composed of multiple individual cells that filled with waste, backfilled with sandy soil to enhance removal of any water that may enter the vault, and covered with a concrete roof as illustrated in Fig. 8.

. Intermediate Depth Disposal. Intermediate depth disposal is prepared for relatively high-activity low-level nuclear waste using shafts. The shafts are generally drilled with augers or other drilling methods approximately $300 \mathrm{ft}$. deep. The shafts may need to be lined or left unlined, and of varying depth and diameter depending on soil properties affecting shaft stability and interaction with the waste package as well as the depth to water table and projected travel time between the emplaced waste and water table. The diagram of a intermediate depth disposal using a shaft is shown in Fig. 9.

- Abandoned Mine. The abandoned mine where the natural resources had already been removed have also bcen proposed as useful facilities for LLW replacement. The stability of remained structure should be inspected prior to waste disposal.

\section{(2) SURFACE IMPOUNDMENTS}

Surface impoundments are employed for the permanent disposal of uranium mill and phosphate tailings. They may also be used for the disposal of other semisolid or liquid radioactive wastes from process operations. A schematic structure is shown in Fig. 10. The disposal is achieved by a combination of tailings treatment steps to remove radioactivity constituents prior to emplacement in the impoundment.

\section{(3) GEOLOGICAL REPOSITORIES}

Conventionally, abandoned mines in very deep underground geological formations have been identified as the preferred disposal method for spent fuel, HLW, and TUR. The deep geological repositories (between $2000 \mathrm{ft}$ and $3000 \mathrm{ft}$ below surface and more than 10,000 year safety time) are considered as the best option for dealing with nuclear waste as schematically shown in Fig. 11. The advantages of this method are :

(i) The long distance from the surface provides shielding from direct exposure to the highly radioactive materials contained therein.

(ii) The long distance and the methods used to seal and excavate the areas reduce the probability of contact with the waste in the future and make intentional removal much more difficult. 

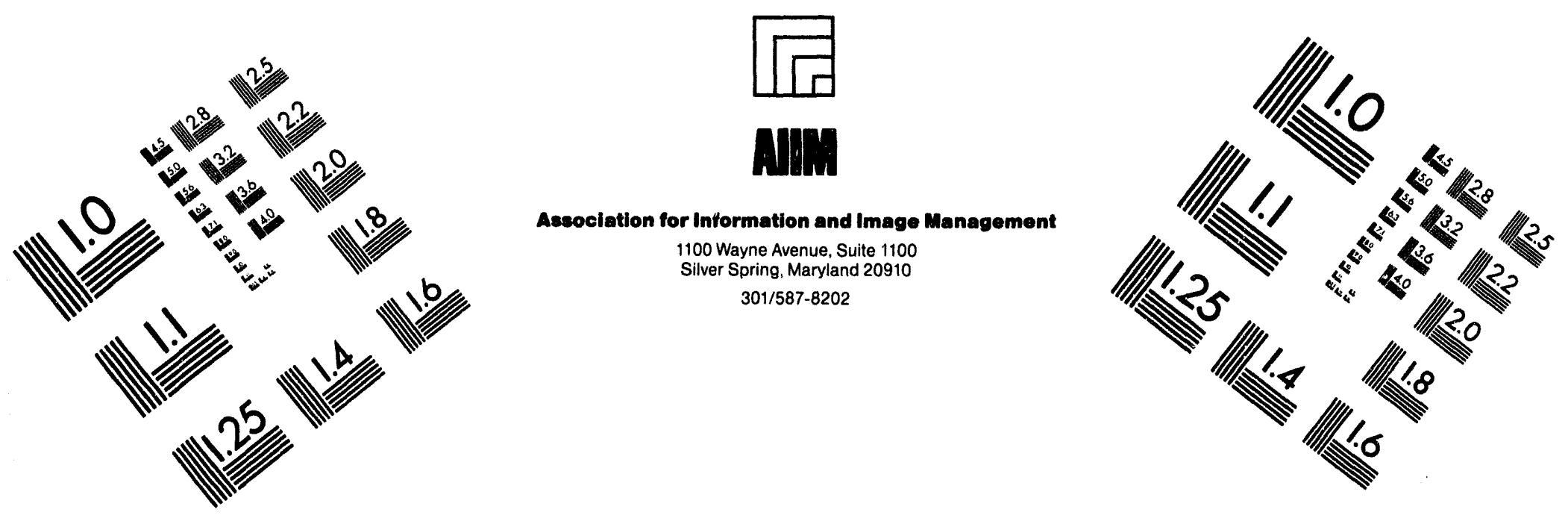

\section{Centimeter}

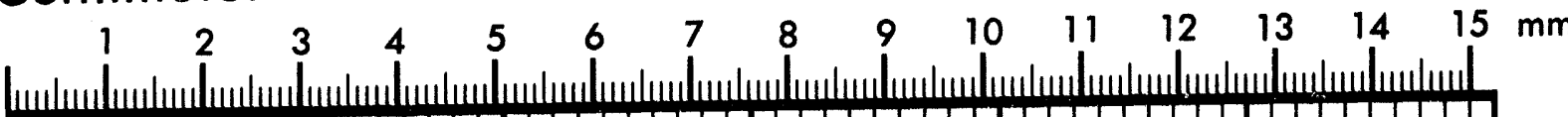

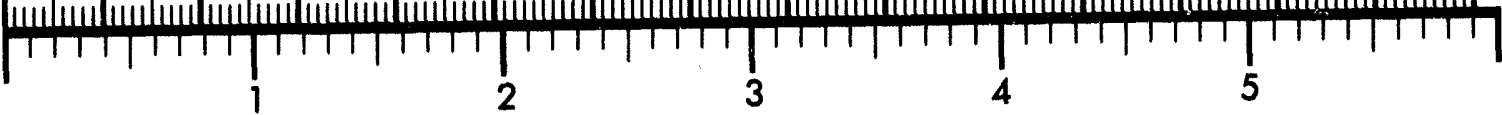
Inches
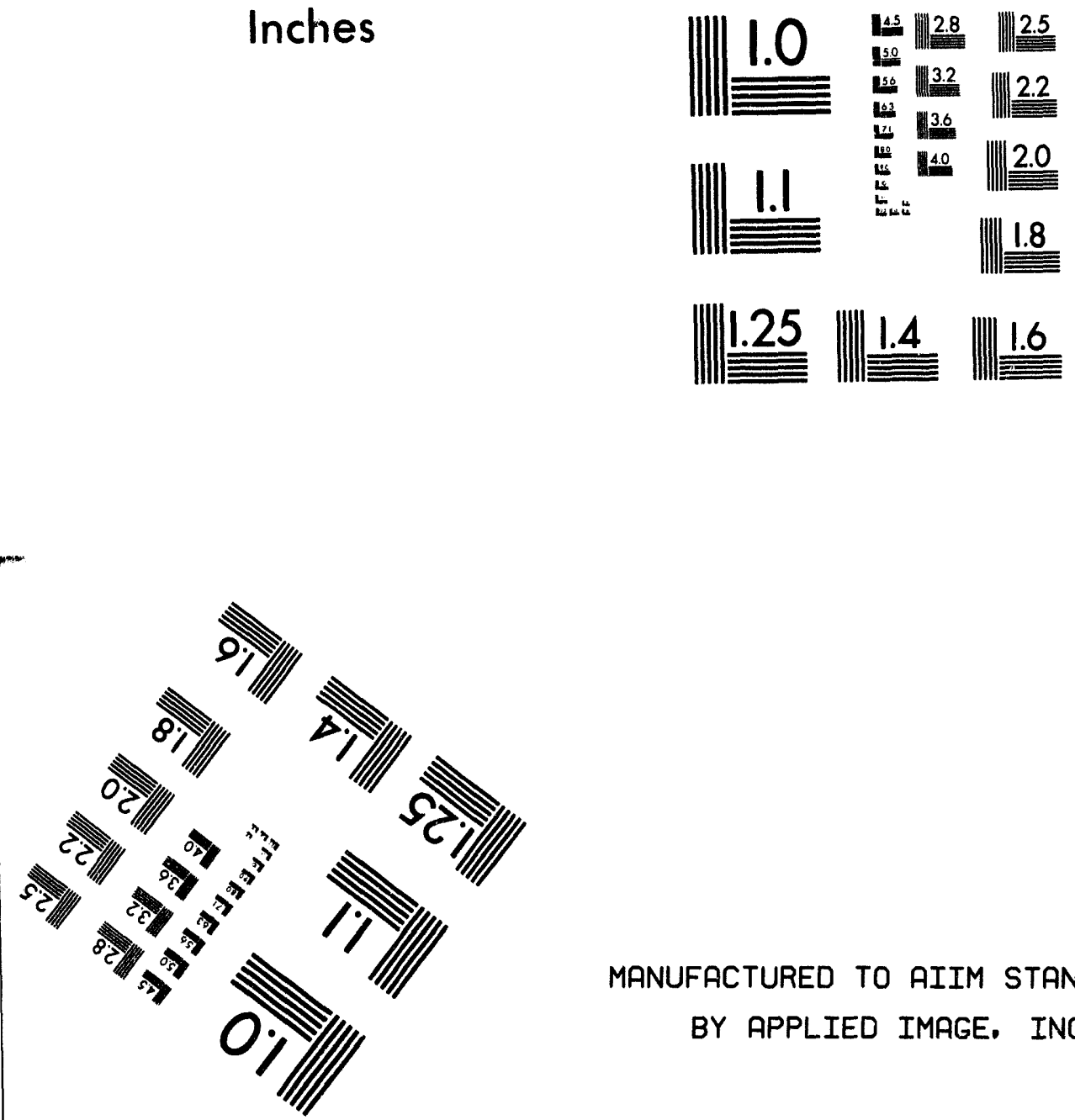

MANUFACTURED TO AIIM STANDARDS

BY APPLIED IMAGE. INC.

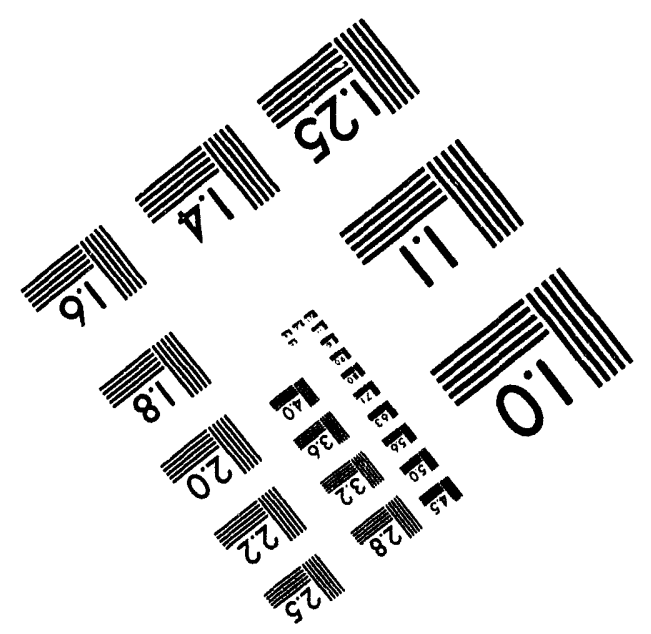



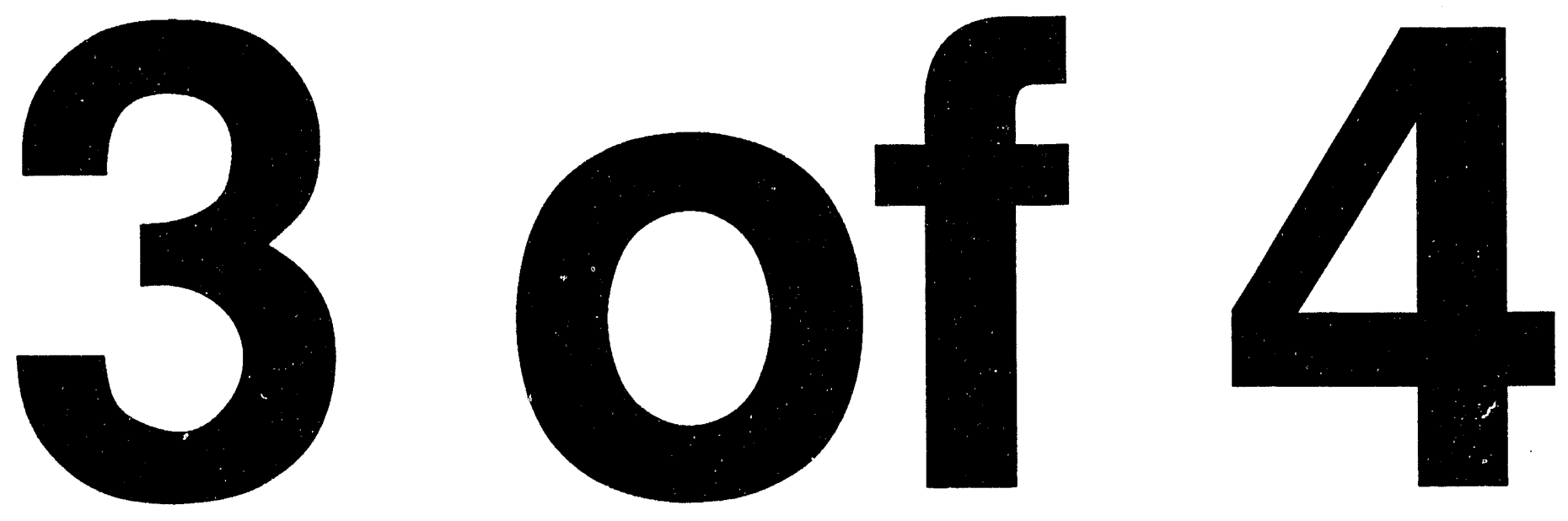


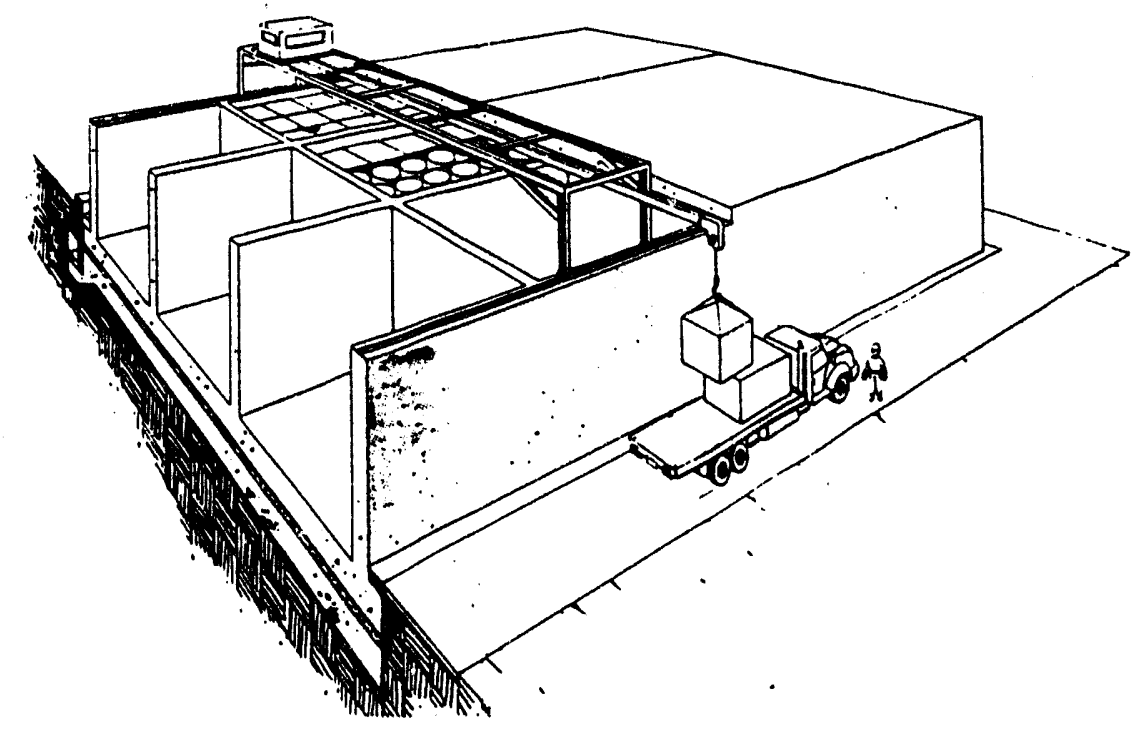

Fig. 8 Schematic of AVG Facility (Source: DOE 1987) 


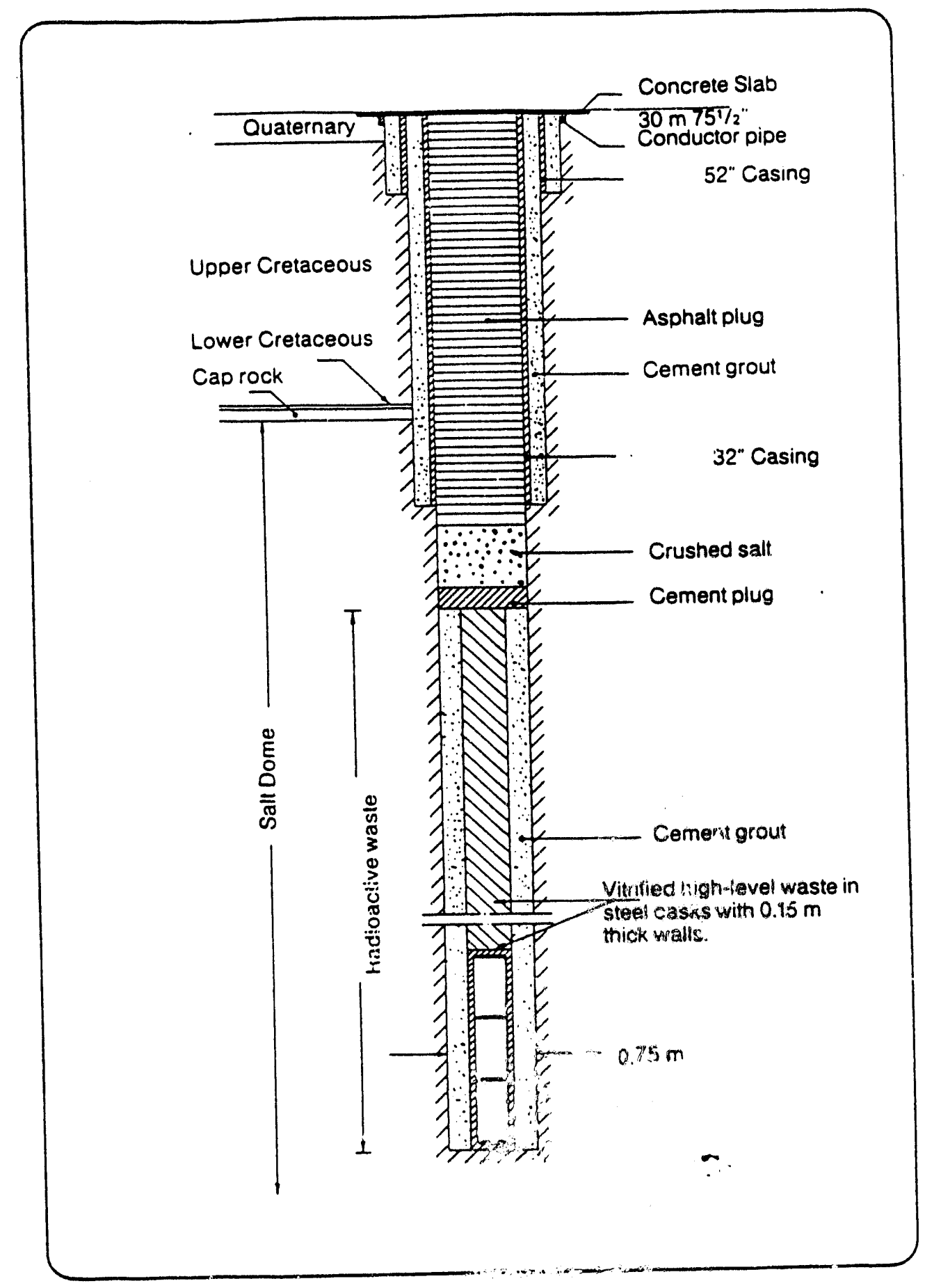

Fig. 9 Diagram of Intermediate Depth Disposal Using Shaft (Source: NEA 1982) 


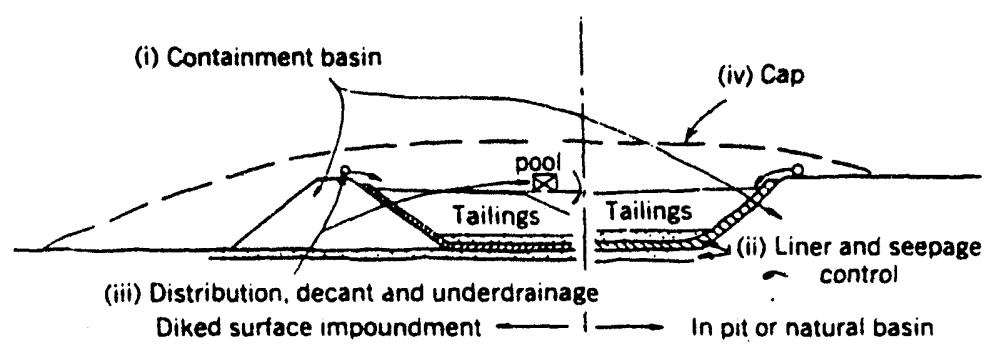

Fig. 10 Schematic Structure of Surface Impoundment Disposal 'Source: NRC 1987) 


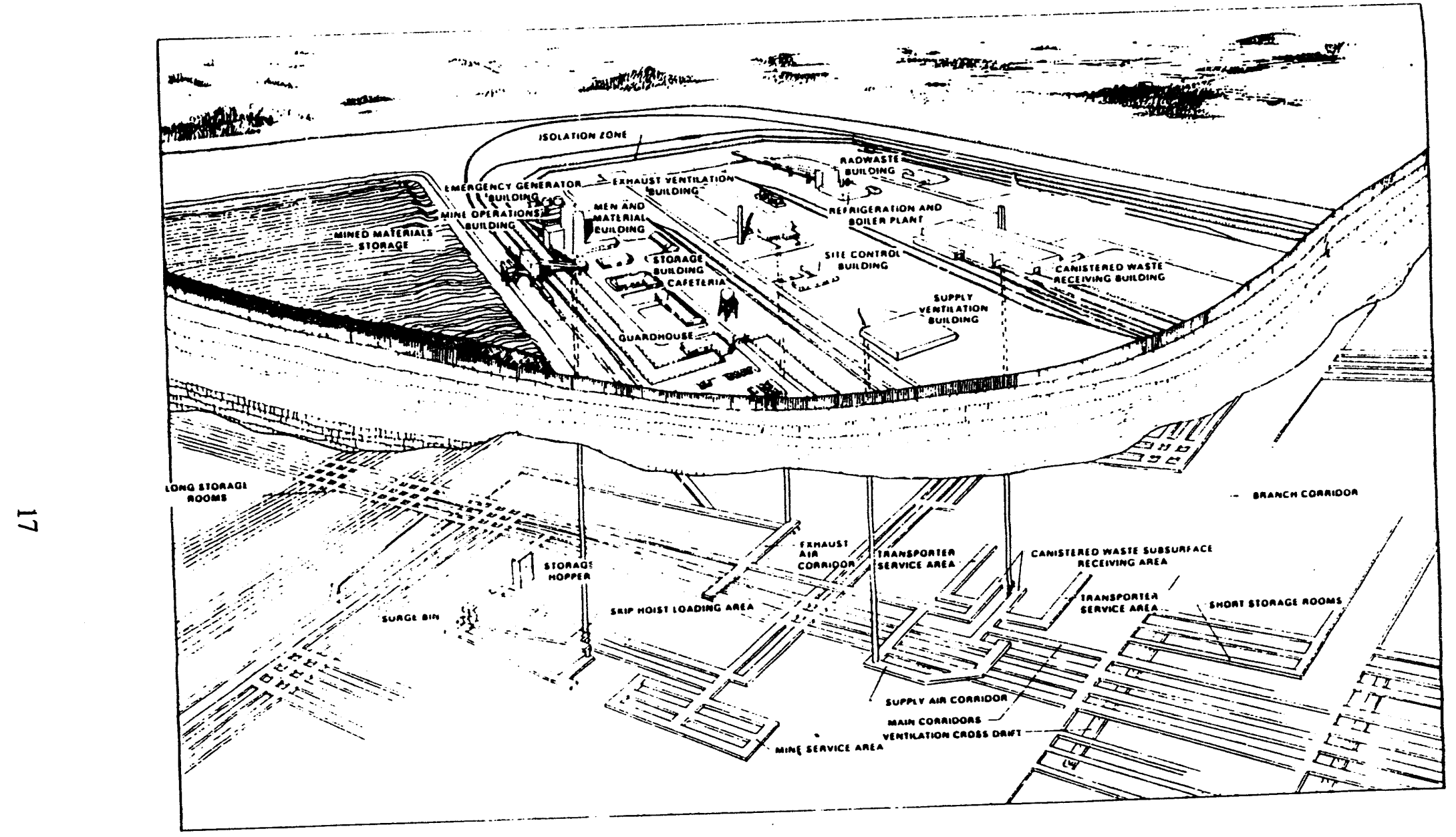

Fig. 11 Schematic Illustration of Geological Repository Disposal (Source: DOE 1987) 
(iii) Site repositories should be located as far away from water as possible. Therefore, the potential exposure via groundwater pathway is minimized.

(iv) Geologic condition is much stable to satisfy the time requirement for waste isolation. Therefore, projections of repository performance during this period can be made with more confidence than could similar projections for other disposal concepts.

Of course, achieving such advantages should take care of the combined effects of several different components of the overall disposal system. The main components are depth of repository below the surface, properties of the host rock, tectonic stability of the area, the hydraulic regime, potential for mining resources from the area, and multibarrier features of the system.

The locations of the geological disposal are limited to those that satisfy the requirements of the site characteristics. Potential geological conditions for federal repositories are salt, tuff or basalt. The potentially acceptable sites for the first and second repositories are shown as Figs. 12 and 13, respectively (DOE, 1984).

\section{(4) UNDERGROUND INJECTION DISPOSAL WELL}

The use of underground wells for liquid waste disposal is a technology originally developed by the oil industry. Injection of liquid radioactive waste into a well is as a possible alternative or complement to a geological repository for portions of the waste stream. Both high-level waste and remote -handled transuranic waste are candidates for this type of disposal because the wastes must be in liquid form, relatively small in volume, and require long-term isolation.

- Deep Well Injection. Deep well injection involves pumping acidic liquid waste to great depth (up to $16,000 \mathrm{ft}$ ). The receiving area is a porous or fractured rock area such as a depleted hydrocarbon reservoirs or zones of natural or induced fractures.

- Shale Ground Injection. Shale grout injection involves pumping neutralized liquid waste or an irradiated fuel slurry mixed with cement, clay, and other additives waste into impermeable shale formation. High pressure water is used to initiate fracture in the shale (hydrofracture).

\section{OCEAN DISPOSAL}

This method has been used and proposed of disposal both LLW and I'LW. 


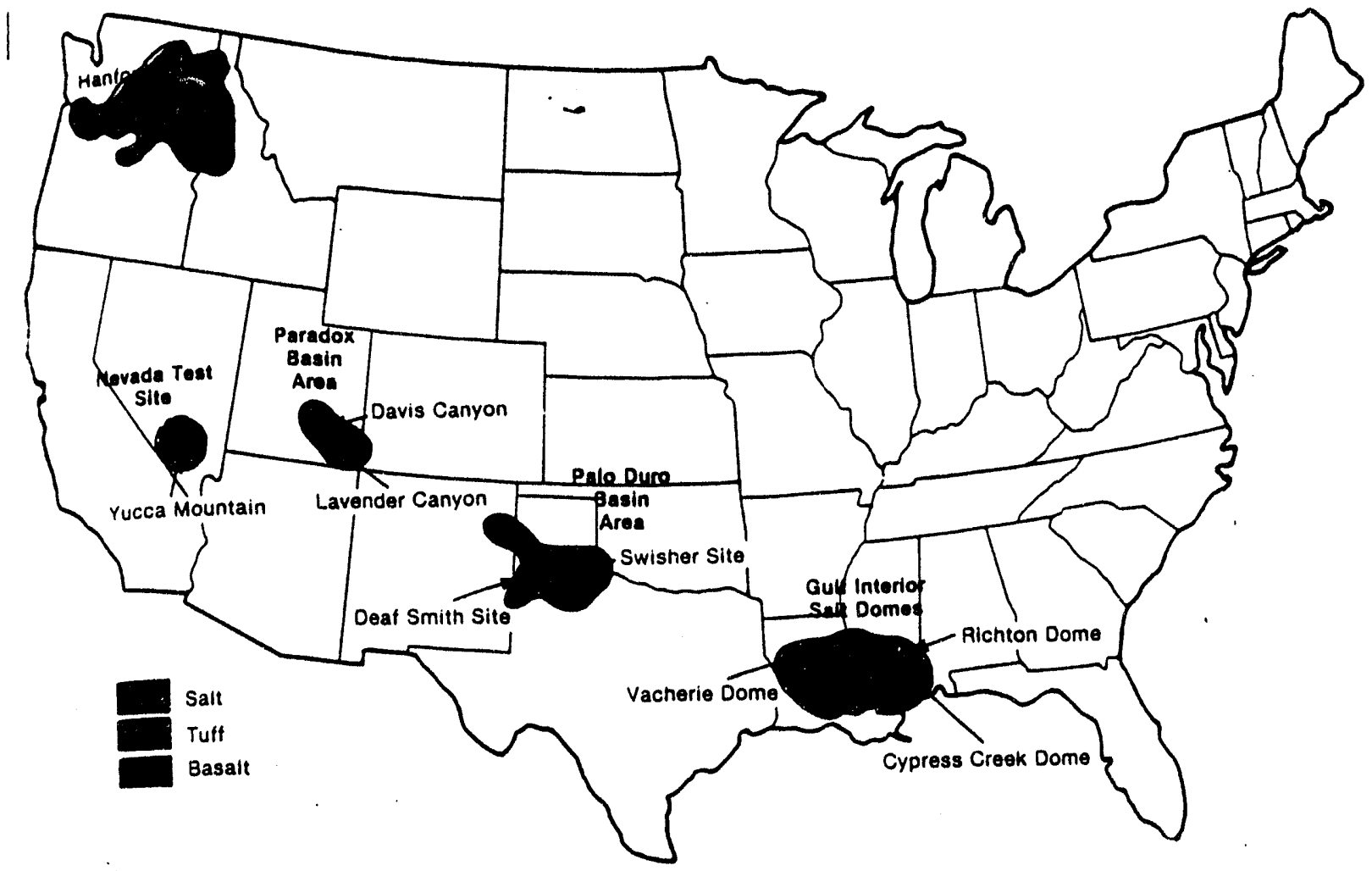

Fig. 12 Location of Candidate Sites for First Geological Repository (Source: DOE 1984)

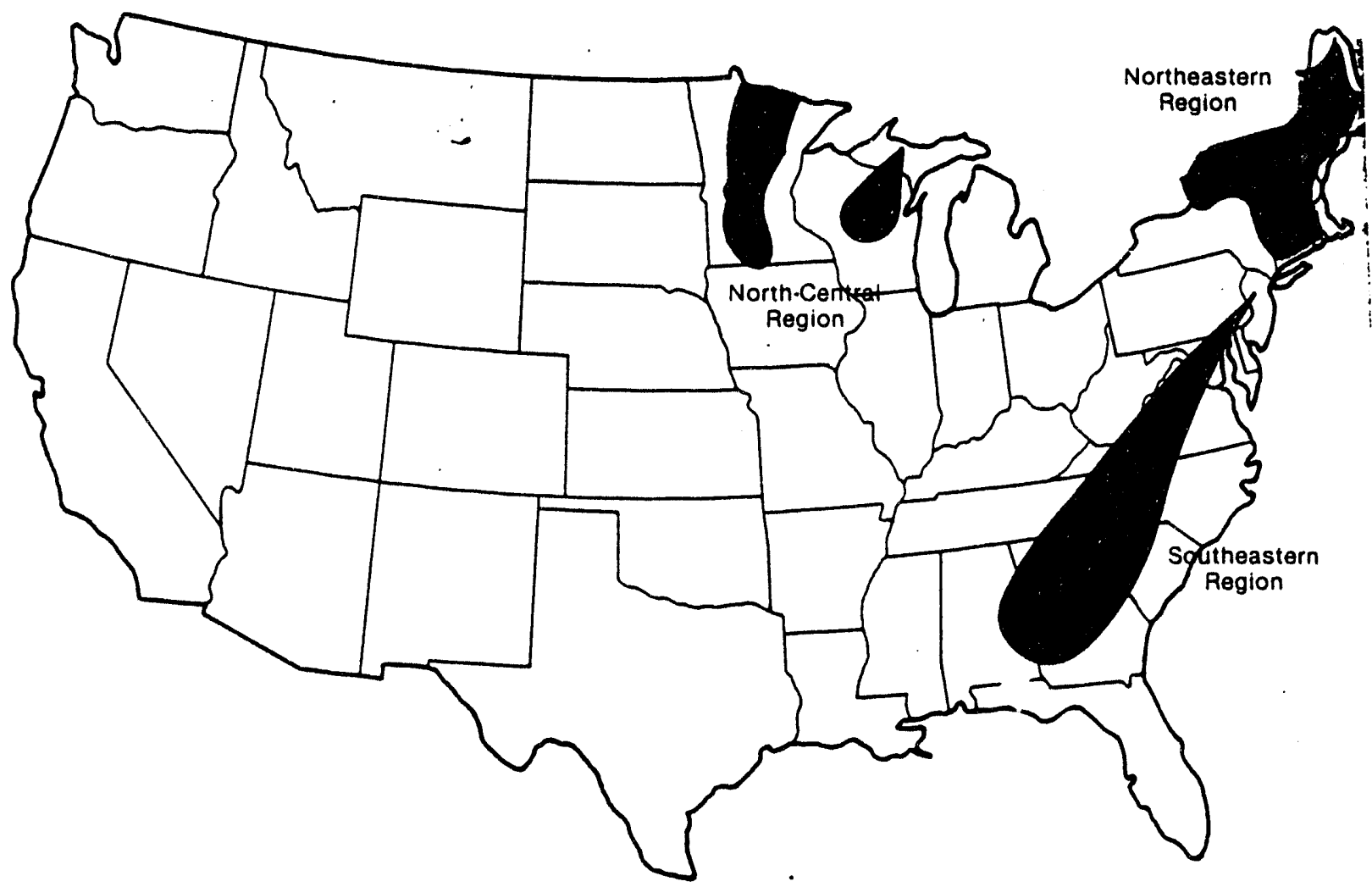

Fig. 13 Location of Candidate Site for Second Geological Repository (Source: DOE 1984) 
. Ocean Disposal of Low-level Waste. LLW was disposed at five sites in both the Atlantic and Pacific Oceans from 1946 to 1969 . The wastes were primarily produced from DOE and defense projects. In general, the material was incorporated into a cement matrix within 55-gallon steel drums. The drums were emplaced on the ocean bottom. However, it is not expected that this technology will be employed again in this country.

- Subseabed Disposal. Subseabed disposal continues to be investigated as an alternative of land-based geological repository for long-term isolation of HLW, spent fuel, and other similar wastes. The waste is disposed in sedimentary rocks on the ocean floor. Several methods are shown in Fig. 14. The practice of this technology is not expected to be performed until after the turn of the century.

\section{(6) SPACE DISPOSAL}

Fundamental to the concept of space disposal of radioactive waste is the ability to remove the material from the earth by placing it into orbit around the sun where it would remain for about one million years as shown in Fig. 15. The cost of handling and transporting the wastes in space is much higher than for land-based alternatives.

\section{(7) OTHER DISPOSALS}

Several other disposal technologies have been proposed as alternatives to conventional geological repositories such as the ice sheets, very deep hole disposal, rock melt with liquid or slurry forms of high-level waste, and transmutation of very long-lived radionuclides into shorter lived or stable isotopes. They were determined to require much more technological development and in some case to provide lower safety margins than would be achievable at a geologic repository.

\section{TECHNOLOGIES WITH POTENTIAL FOR APPLICATION TO DUST/VAPOR CONTROL AT NUCLEAR WASTE SITES}

Although no literature has been found so far to describe how to control the dust/vapor generated from the in-situ nuclear waste handling, a few treatment technologies have been demonstrated for HLW and LLW under DOE and EPA sponsorships. Some of the treatment technologies may have the function of the dust/vapor suppression as they are performed. 


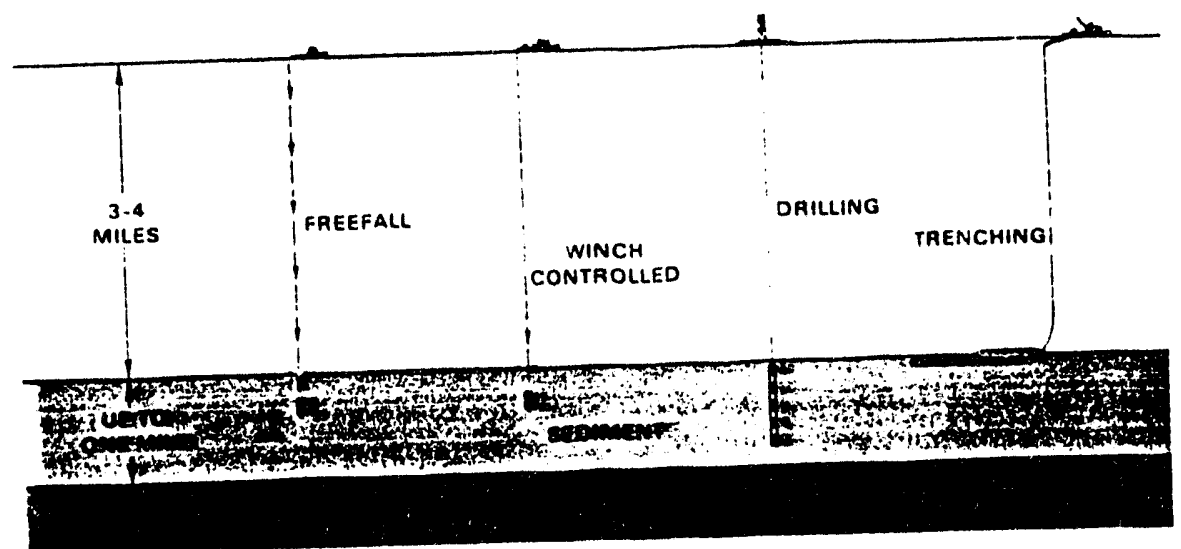

Fig. 14 Several Proposed Methods for Emplacing Waste in Subseabed Repository (Source: DOE 1980)

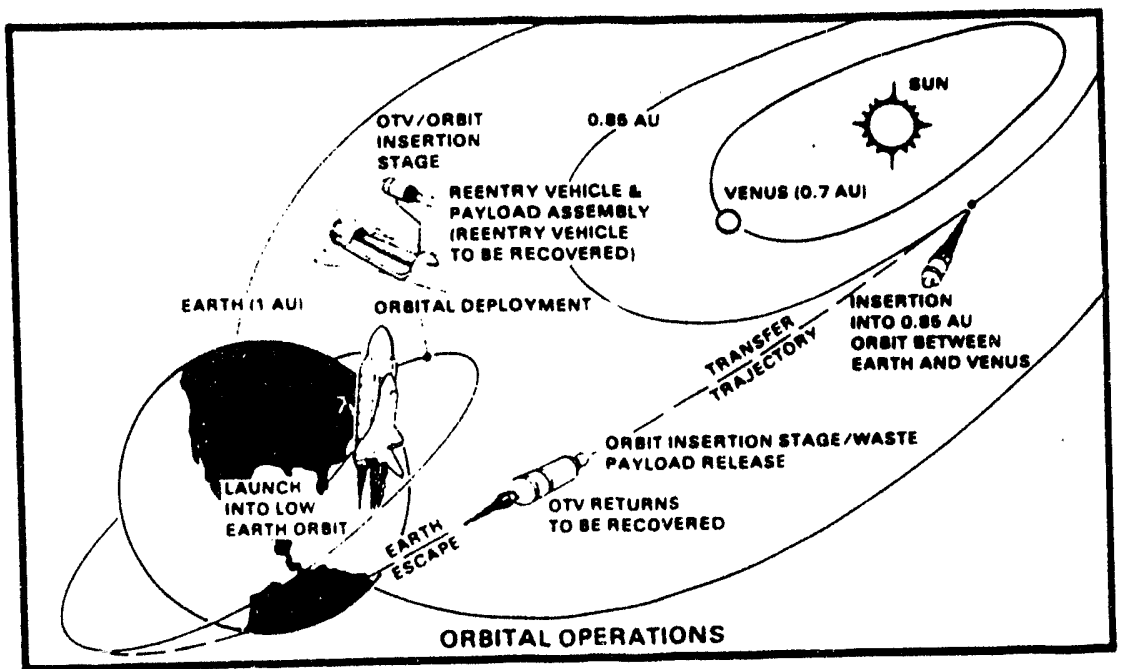

Fig. 15 Orbital Transfer Operations for Space Disposal (Source: DOE 1980) 
Technologies which are using for management of HLW and LLW are shortly introduced as follows:

\subsection{TECHNOLOGIES FOR TREATMENT OF HLW}

\section{(1) VITRIFICATION}

Vitrification involves the melting of soil or other contaminated solid medium and its subsequent freezing into an obsidian phase. The materials contaminated are trapped within the obsidian-like treated material and are stabilized. The high temperatures required for initially melting the soil allow for the thermal destruction of any organic contamination present. The simultaneous destruction of organic contaminants and stabilization of metal contaminants allows for the complete treatment of a solid waste in one step (Bickford, 1993; Staley, 1993).

The vitrification technology is being employed at three high level waste sites (Idaho, Hanford and Savannah River) which are managed by DOE and at one state-owned facility (West Valley, NY). The vitrification facilities at Savannah River and West Valley sites have been completely constructed and are undergoing operational check-out prior to start-up. The vitrification program for Hanford and Idaho high level wastes are in the front-end development work. Pretreatment processes include ion-exchange, sludge dissolution, precipitation, sludge washing and other developmental processes designed to minimize volume and maximize waste loading of the final waste form destined for disposal in the national repository.

However, some researchers believe that radionuclides are not destroyed in the hightemperature treatment process (Staley, 1993). The ratio of radioactivity to the resultant mass is much higher than the pretreatment. High-temperature treatments nature of vitrification can cause pollution problems such as high nitrogen oxide and metal emission or even grater increase in leachability for toxic metals (probably greater because they are converted to the oxide form, which is leachable). If the vitrification technology is used for low level nuclear waste treatment, the final waste form after the treatment may change its classification to transuranic or greater than low level waste. It is costly because it is power intensive. However, risk is higher due to dust/vapor generation if this technology is used in in-situ waste sites and the waste in its final form is to be excavated and transformed to the national repository. 


\section{(2) IMMOBILIZATION//SOLIDIFICATION}

if the nuclear waste is in the form of high-level liquid radioactive waste (HLLW), immobilization/solidification technology can be used to minimize the amount of high-level waste by transforming liquid wastes into cement waste (Poulson, 1993). The remaining sludges would then be formed into glass logs (as high-level waste) for eventual disposal in the national repository. The West Valley high-level liquid waste is arranged to use immobilization/solidification technology. However, concerning the environmental aspect, this technology still face challenges which include removal of the liquid waste from the original storage, development of cement and glass waste forms except for controlling the dust/vapor generated during the excavation or transportation processes.

\section{(3) COMPACT PROCESSING UNITS (CPUs)}

The Compact Processing Units (CPUs) is developed by the Pacific Northwest Laboratory of Waste Treatment Technology Department. This device is expected to treat high-level liquid nuclear wastes in close proximity to tanks (Ballinger et al., 1993). The CPUs consists of four major subsystems: an ion exchange process, a process control system, an enclosure/containment area and a tank farm interface. The relative small and portable CPUs are self-contained and can be easily deployed as single unit or in a series as needed.

The ion exchange process separates the cesium from the incoming tank waste and returns the low-level cesium waste stream to a storage tank. The ion exchange columns store the cesium until it can be collected as high-level wastes. The concentration of cesium remaining in the low-level waste stream will be less than the U.S. Nuclear Regulatory Commission (NRC) Class A limit of $<1 \mathrm{ci} / \mathrm{m}^{3}$. The capacity of the CPUs will be designed at 8 to 20 liters per minute. The CPUs is expected to be completed by the end of 1996 .

\section{(4) ISOLATION}

An overall isolation technology for high level nuclear waste treatment is currently being developed by a WVU Team funded by DOE. The concept of this innovative technology is trying to modify longwall mining method with a robotics excavating system to cut a space at suitable level below the nuclear waste site. Permanent lining/sealing will be installed to isolate the nuclear waste site from the uncontaminated zone.

This technology using underground access and remote excavation will reduce the potential effects to environments and on-site operators. It is anticipated that the best advantage of the isolation technology is to stabilize the nuclear waste without disturbing the 
waste site itself. But a few critical problems are still being worked on, such as design of the permanent lining/sealing system, cutter bit selection, mining horizon, detection and repair of robotics system, dust/vapor suppression due to rock cutting/crushing, underground ventilation, etc.

\subsection{TECHNOLOGIES FOR TREATMENT OF LLW}

Most of the low-level nuclear waste sites under EPA and DOE are treated similarly as the hazardous waste sites. EPA has provided greater offers to manage these types of waste. Based on the EPA studies under the Innovative Technologies in the Emerging Technology Program and the Innovative Technologies in the Demonstration Program, lowlevel nuclear wastes can be treated by using some similar technologies for hazardous waste treatment depending on the characteristics of the specific wastes. The equipment for material handling under various operations (i.e. excavation, transportation, processing, loading and storage, and personal protection) have been reported in the third quarter report. The nineteen treatment technologies and their potential applications are emphasized in the fourth quarter report. All of them are divided into five categories in the Innovative Technologies in the Emerging Technology Program, which include thermal destruction [8], biological degradacion [13], physical/chemical treatment [26], solidification and stabilization [2], and material handling [4], and eight categories in the Innovative Technologies in the Demonstration Program, which include thermal destruction [9], biological degradation [17], physical/chemical treatment [34], solidification and stabilization [11], radioactive waste treatment [2], thermal desorption [16], material handling [3], and other [1] according to the technology applications in the projects (EPA, 1993). The number in the square brackets denote the numbers of project using the classified technologies.

\subsection{DUST/VAPOR CONTROL IN NUCLEAR WASTE SITES}

Although most technologies mentioned above have been demonstrated, the majority of those demonstrations were performed in the off-site facilities. In other words, the nuclear waste handling (i.e. excavation, transportation, loading/unloading, stockpiling/storage, etc.) should be provided prior to applications of these technologies. Therefore, just like the excavation of in-situ hazardous wastes, the dust/vapor generation can not be avoided during in-situ nuclear waste handling if no proper suppression method is applied, and it will be more 
harmful to human and environments than that of the hazardous waste sites.

According to the literature reviews on dust/vapor control for in-situ hazardous waste handling last year, more than a dozen commercial available suppression technologies have been identified. These technologies are divided into three groups regarding the procedures of in-situ material handling. Before excavating the hazardous wastes, in-situ treatment and acid gas neutralization additives for vapor control can be applied. Most of the control methods, such as control agents, foam suppressions, surface enclosures, self-supporting enclosure, covers, mats and membranes, vacuum trucks, and windscreen process can be used for the excavation of in-situ hazardous wastes. Lining system, such as covers, mats and membranes are also required for long/short term stockpiling/restoration after the hazardous wastes have been excavated. In addition, it is strongly recommended to use the excavating equipment for dust/vapor control since this type of control method is considered an effective active approach instead of other passive mitigation methods. It is still questionable whether or not these methods can be directly transformed to dust/vapor suppression for in-situ nuclear waste handling, especially for high level nuclear wastes since access of nuclear waste sites and on-site operation by human are very much riskier than working on no-nuclear waste sites.

So far, there is no commercially available technology for in-situ nuclear waste handling. But some devices introduced in the fourth quarterly report may be used for nuclear dust/vapor control. Those are:

\section{(1) COMPUTERIZED EXCAVATOR}

The computerized excavator, EX 300-2 the latest model is produced by Hitachi Company in 1992. This type of excavator can be operated easily by the driver on on-site operations and exhibits better performance since the electronic total control system is installed on the excavator.

\section{(2) REMOTE CONTROLLED EXCAVATOR}

It was the first generation of the remote controlled excavator (Model $690 \mathrm{C}$ ) by John Deere Company in 1988. This excavator provides off-site operations with radio transmission, coaxial cable, or fiber-optic cable resulting in maximum worker safety.

\section{(3) PNEUMATIC EXCAVATOR SYSTEM}

Although the pneumatic excavation system is currently under patent (Worlko, 1992), 
the concept of the system addresses the fugitive dust and gas emissions because the transported materials are trapped and enclosed in the system preventing discharge of dust, speed of excavated fragments, and run-off of toxic liquid.

The following three improved or innovative technologies may also have potential for effective control of dust/vapor for handling of in-situ nuclear waste sites if some modifications can be done in the future.

\section{(4) HORIZONTAL WELLS FOR ENHANCED ACCESS TO THE SUBSURFACE}

Most nuclear waste sites, especially for high level waste sites are inaccessible. Horizontal walls have been demonstrated to improve access to the subsurface (Kaback, 1993). Thus it maximizes the effectiveness of the remediation technologies and allows characterization and remediation of sites previuusly inaccessible. Because contamination is generally found in vertically limited and horizontally extended zones, the horizontal geometry exposes the maximum screen area to zone of interest. Over one hundred horizontal wells have been installed across the U.S. in environmental applications. Most wells have been installed in shallow depths ( $<30 \mathrm{ft}$.) for pump-and-treat or vapor extraction remediation. It is expected that using this method can minimize the dust/vapor effects on environments, but there are a few areas that need improvements including well material selection for horizontal installation, well stability, sealing or grouting of abandoned wells, etc. Some technologies of underground mining can be transformed to improve these shortcomings.

\section{(5) TELE-OPERATED REMOTE CONTROL EQUIPMENT (TORCE)}

Another way to access the nuclear waste sites is to use the Tele-Operated Remote Control Equipment (TORCE). By using this equipment, the on-site operation can be avoided, and potential risk to human can be reduced (Bode, 1993).

Two types of remote control equipment have been developed by John Deere Dobuque Works. The $690 \mathrm{C}$ All Terrain Excavator was the first unit and used the radio system by Vectran Corp. in Pittsburgh, PA. The 690 D LC is a second generation unit on tracks with micro-wave communications link which can retrofit remote control capabilities to virtually every current model of John Deere excavator.

A TORCE kit consists of several components, including remote control station, removable vehicle remote system, and adaptor kit. Remote control station is contained in a rugged environmentally sealed container that can be closed to protect the video monitor, switches and controls from shown in Fig. 16. In the open operating mode, all 


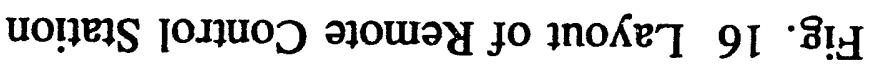

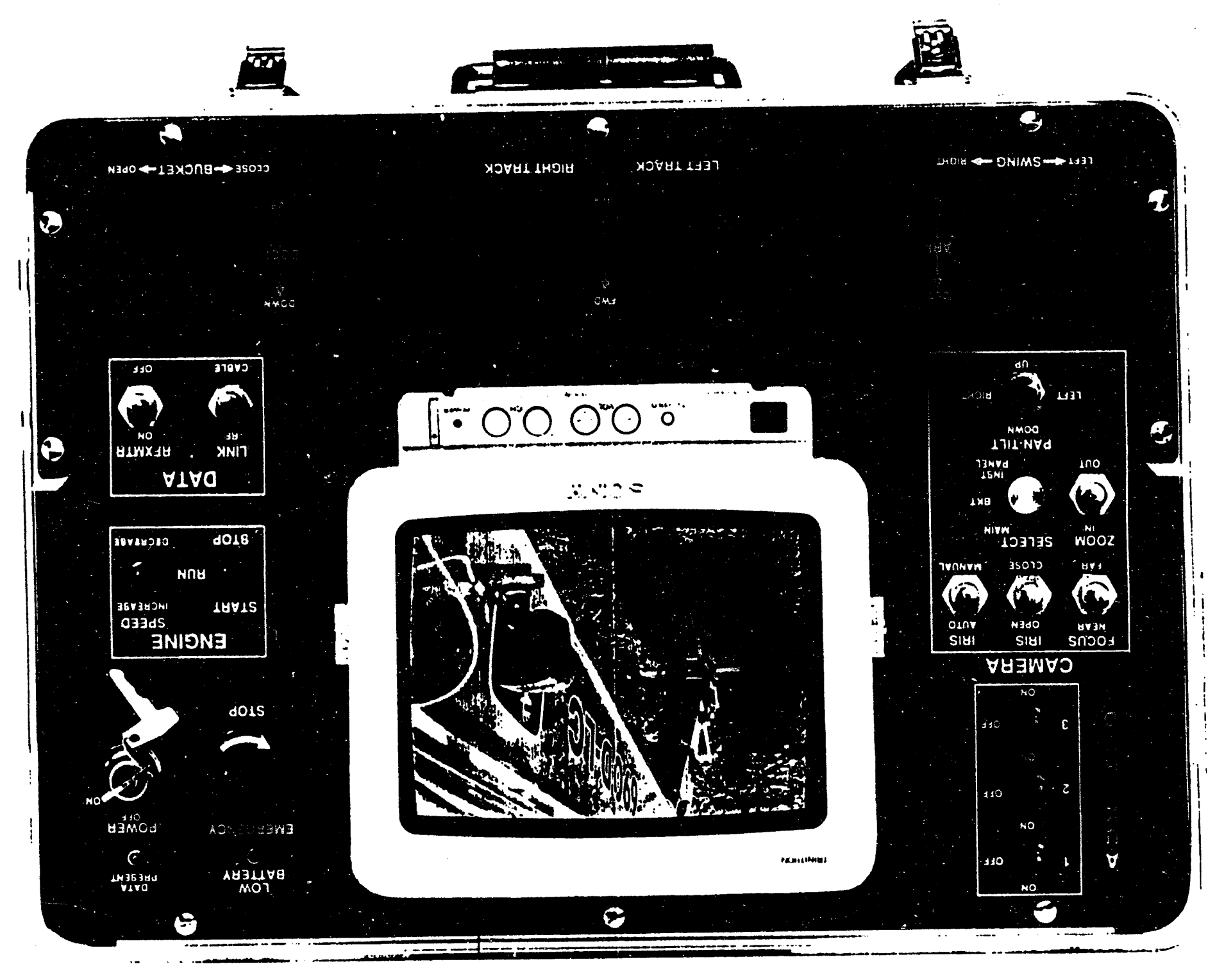


electronics are environmentally sealed from moisture, dust, and foreign materials. The working equipment, including boom, arm, bucket and swing, is controlled by two fourfunction levels. Discrete (on/off) control is adequate for all other functions: dozer blade up and down, stabilizers up and down, engine start and stop, engine speed increase and decrease, travel speed high range and low range, auxiliary work tool (hydraulic hammer) and bucket clamp open and close. The 7-in. high-resolution color monitor is provided with views from any of the three cameras mounted on the excavator. The remote control operation station can operates for eight hours minimum on a set of rechargeable batteries. The station can be connected to a 24 VDC or 110/220 VAC; 50 or $60 \mathrm{~Hz}$ source for continuous operation and/or battery charging. By using this station, an operator can remotely run an excavator with or without direct visual contact from distance up to one mile or more away in all weather and terrain conditions. It can be quickly installed on almost every model of John Deere excavator now in service. Even after the TORCE kit is installed, the excavator can be operated manually. It is also documented that the TORCE productivity is increased and costs reduced when compared to other hazardous material-handling system requiring on-site personals encumbered with encapsulating suits.

\section{(6). HYDROPERMUTATION SYSTEM (Hydrop)}

The hydropermutation system combines the actions of a conventional wet scrubber and a rotational atomizer (Leang and Isaacs, 1993). Based on testing data from various plants, the hydropermutation systems shows its effectiveness to remove particles and corrosive air pollutants, such as $\mathrm{SO}_{2}$ (removal efficiency: $>97 \%$ ), and even radioactive particles (removal efficiency: $>99 \%$ ). The hydropermutation system comprises a water jet and a rotary impeller (atomizer) within a multisided chamber. The concept of this device is based on atomization and impaction for particle and gas removal. Water is injected simultaneously with flue gases into the Hydrop system. The size of the water droplets and particles are reduced by impaction and atomization in this highly turbulent chamber, and are controlled by the speed and configuration of the atomizer. An improvement of Hydrop is the modular design with that it can be easily adapted to most existing air pollution control systems and serves as enhancer to satisfy the new air emission standards. Effluent generated by the system can be treated and reused. From the point of view for dust/vapor control due to insitu nuclear waste handling, this technology may have potential to combine with either the enclosure method or tele-operated remote control equipment to improve these technologies and protect the environments better. 


\section{FUTURE WORK}

The search for dust and vapor suppression technologies during excavation will be continued. The types of contaminants and mode of transport (vapor or dust) and migration concern during cxcavation of hazardous wastes will be summarized. Currently available dust and vapor suppression technologies for the excavation of contaminated soils, sludges and sediments will be reported. Specific case studies will be selected to illustrate the decontamination methods used and strategies implemented for the specific hazardous inorganic/organic and nuclear wastes. 


\section{REFERENCE}

ASCE 1982, Nuclear Waste Management. American Society of Civil Engineers, New York, New York 10017, 49 pp.

Ballinger, M.Y. K.P., Brooks, D.R., Jackson, J.H. Konynenbelt, and W.G. Richmond, Waste Treatment Technology Department, Pacific Northwest Laboratory, Presented at the I\&EC Special Symposium, American Chemical Society, Atlanta, GA, September 27-29, 1993, pp. 1031-1032.

Bickford, D.F., 1993, "Waste Vitrification Program at the Savnnah River Site," Westinghouse Savannah River Co. Presented at the I\&EC Special Symposium, American Chemical Society, Atlanta, GA, September 27-29, 1993, pp. 51-52.

Bode, B. D., 1993, Sales and Special Engineering of John Deere Dubuque Works, Personal Communication.

DOE 1980, "Management of Commercially Generated Radioactive Waste," Final Environmental Impact Statement, DOE/EIS-0046F, U. S. Department of Energy, Washington, D.C., October 1980.

DOE 1983, "Spent Fuel and Radioactive Waste Inventories, Projections, and Characteristics." DOE/NE-0017/2, U.S. Department of Energy, September 1983.

DOE 1984, "Draft Mission Plan for the Civilian Radioactive Waste Management Program," DOE/RW-0005 DRAFT, April 1984.

DOE 1987, "Alternative Concepts for Low-Level Radioactive Waste Disposal." DOE/LLW60, Conceptual Design Report, Prepared by Rogers \& Associates Engineering Corporation for EG \& G Idaho, Inc. and the U. S. Department of Energy, June 1987.

EPA, 1993, "The Surperfund Innovative Technology Evaluation Program: Technology profiles Fifth Edition." Risk Reduction Engineering Laboratory, EPA/540/R-92/077, 388 pp.

John, H. G., 1985, "Management of the Nation's Commercial High-level Waste Radioactive Waste," Washington, DC, U.S. Congress, Office of Technology Assessment, OTA-0-171, $343 \mathrm{pp}$.

Kaback, D.S., 1993, "Horizontal Wells for Enhanced Access to the Subsurface," Colorado Center for Environment Management, Presented at the I\&EC Special Symposium, American Chemical Society, Atlanta, GA, September 27-29, 1993, pp. 240-242. 
Leung, S.W. and G.P. Isaacs, 1993, "An Innovative Air Pollution Control Design for Particles and Flue Gases," Idaho State university, Presented at the I\&EC Special Symposium, American Chemical Society, Atlanta, GA, September 27-29, 1993, pp. 922-923.

IAEA 1981, "Shallow Ground Disposal of Ra lioactive Waste - A Guidebook," Safety Shis No. 53, International Atomic Energy Agency, Vienna, 1981, 52 pp.

NEA 1982a, Disposal of Radioactive Waste. Nuclear Energy Agency, OCDE/OECD, paris $1982,53 \mathrm{pp}$.

NEA 1982b, Geological Disposal of Radioactive Waste, OECD 1982, 54 pp.

NRC 1979, Proposed Procedures for Siting and Licensing A High Level Waste Geologic Repository, 10 CFR Part 60, U.S. NRC, 44FR 70408, December 1979.

NRC 1982, "Final Environmental Impact Statement on 10 CFR Part 61, Licensing Requirement for Land Disposal of Radioactive Waste." NUREG-0945, November 1982.

NRC 1992, Radioactive Waste Repository Licensing, National Research Council, National Academy Press, Washington, D. C. 1992, 96 pp.

NRC 1985, "Alternative Methods for Disposal of Low-Level Radioactive Wastes," NUREG/CR, Vols, 2-5, Below Ground Vaults, Aboveground Vaults, Earth Mounded Concrete Bunkers, Shaft Disposal, Prepared for the U.S. Nuclear Regulatory Commission by the U.S. Army Engineer Waterways Experiment Station, October 1985.

Poulson, W.G., 1993, "High-level Waste Management at the West Valley demonstration Project: A Driving Force for New Technology Development," West Valley Nuclear Services Company. Inc. Presented at the I\&EC Special Symposium, American Chemical Society, Atlanta, GA, September 27-29, 1993, PP. 46.

Robert, E. B. and Catherine C. S., 1989, Radioactive Waste Management, John Wiley \& Sons, $435 \mathrm{pp}$.

Staley, L.J., 1993, "Vitrification Technologies for the Treatment of Contaminated Soil," U.S. DOE, Presented at the I\&EC Special Symposium, American Chemical Society, Atlanta, GA, September 27-29, 1993, PP. 498-499. 
Chemical Destruction of Polychlorinated Biphenyls (Project MC-6)

\title{
Quarterly Report for Period
}

for Period October 1 through December 31, 1993

Work Performed Under Contract

No.: DE-FC21-92MC29467

\author{
For \\ U.S. Department of Energy \\ Office of Fossil Energy \\ Morgantown Energy Technology Center \\ Morgantown, West Virginia
}

\author{
By \\ Kung K. Wang \\ Department of Chemistry \\ West Virginia University \\ Morgantown, West Virginia
}

February 1994 


\begin{abstract}
The general objective of this research is to first evaluate the existing technologies for destruction of polychlorinated biphenyls (PCBs) by chemical methods. Development of new chemical treatment procedures for dechlorination of PCBs will also be a major focus of this research project. The detailed reaction pathway for the dechlorination process will be carefully probed so as to gain fundamental understanding of the reaction mechanism. Such information will be invaluable in providing guidelines for designing an efficient and economical system.

In the first part of this report, the main focus will be on the assessment of the current technologies and the discussion of the basic chemical reactions behind these treatment procedures. The commercial processes as well as procedures recently published in the literature will be reviewed, including dechlorination of $\mathrm{PCBs}$ by sodium and other alkali metals, by the use of strong base, by catalytic dechlorination, and by photochemical degradation.

In the second pirt of this report, experimental results obtained from dechlorination of aromatic halides, including chlorobenzene, 4-chlorobiphenyl, Aroclor 1242, and Aroclor 1254, by sodium 1-dimethylaminonaphthalide (NaDMAN) will be discussed. The easy recovery of 1 dimethylaminonaphthalene from the reaction mixture by a simple acid extraction offers a significant advantage for its use as an electron carrier for sodium metal during the dechlorination process. However, as a reagent NaDMAN in tetrahydrofuran is not very stable. It is necessary to prepare NaDMAN at $-10^{\circ} \mathrm{C}$ and immediately prior to use. Storing NaDMAN solution at room temperature resulted in gradual loss of its reactivity over several days.

In the third part of this report, research efforts toward developing sterically hindered triarylboranes as potential electron carriers for sodium metal will be discussed. Because of the presence of an empty p-orbital on the boron atom which allows an electron from sodium metal to reside over it, triarylboranes are excellent electron carriers. Moreover the corresponding radical anions are stable over an extended period of time at room temperature, allowing dechlorination procedure to be carried out at ambient temperature. Attempts are being made to try to incorporate an amino group into the chemical structure of sterically hindered triarylboranes with the intention for easy recovery after the dechlorination process is complete. Attaching a suitable electron carrier to a polymer backbone to allow easy separation is also being investigated.
\end{abstract}


Section No.

Page No.

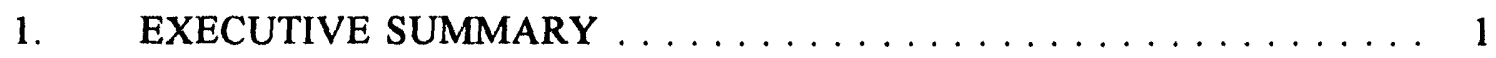

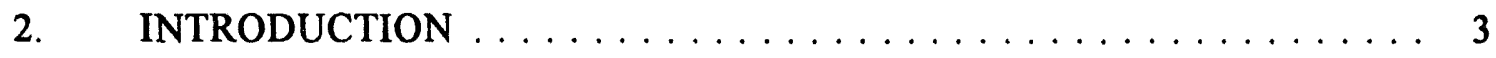

$2.1 \quad$ Background $\ldots \ldots \ldots \ldots \ldots \ldots \ldots \ldots$

2.2 Destruction of $\mathrm{PCBs} \ldots \ldots \ldots \ldots \ldots$

2.3 Purpose and Scope of Report $\ldots \ldots \ldots \ldots \ldots \ldots$

3. LITERATURE REVIEW OF CHEMICAL DESTRUCTION OF PCBS 4

3.1 Dechlorination of PCBs by Sodium and Other Alkali Metals .... 4

3.2 Destruction of PCBs by the Use of Strong Base . . . . . . . . 6

3.3 Catalytic Dechlorination of PCBs $\ldots \ldots \ldots \ldots \ldots$

3.4 Photochemical Dechlorination of PCBs $\ldots \ldots \ldots \ldots$

4. CHEMICAL DESTRUCTION OF CHLORINATED AROMATICS BY SODIUM 1-DIMETHYLAMINONAPHTHALIDE (NaDMAN). . . . . . . 12

5. SYNTHESIS OF STERICALLY HINDERED TRIARYLBORANES AS POTENTIAL ELECTRON CARRIERS FOR SODIUM METAL . . . . . . 13

6. EXPERIMENTAL SECTION $\ldots \ldots \ldots \ldots \ldots \ldots \ldots$

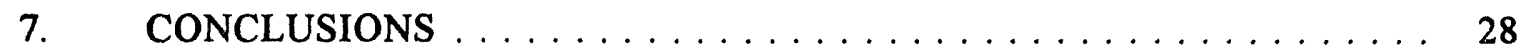

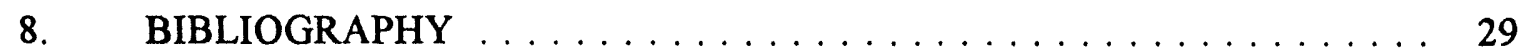




\section{LIST OF FIGURES}

Figure

Page No.

Figure 1. Chemical Structures of Three Representative

Polychlorinated Biphenyls . . . . . . . . . . . . . 3

Figure 2. Dechlorination of Chlorobenzene by Sodium $\ldots \ldots \ldots \ldots \ldots$

Figure 3. Block Diagram of the Trinity PCB Treatment Process $\ldots \ldots \ldots \ldots$

Figure 4. Destruction of PCBs by the KPEG Process $\ldots \ldots \ldots \ldots$

Figure 5. The KPEG Process via the Benzyne Pathway $\ldots \ldots \ldots \ldots$

Figure 6. Preparation of the KGME Reagent $\ldots \ldots \ldots \ldots \ldots \ldots$

Figure 7. Acetone-Induced Photochemical Degradation of PCBs $\ldots \ldots \ldots \ldots$

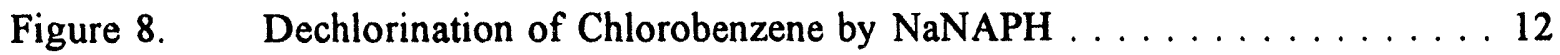

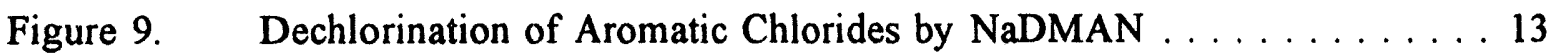

Figure 10. Reaction of Trimesitylborane (20) with Sodium Metal $\ldots \ldots \ldots 14$

Figure 11. 4-(Dimethylamino)phenyldimesitylborane (22) as an Electron Carrier . . 14

Figure 12. Synthetic Procedure for 4-(Dimethylamino)phenyldimesitylborane (22) . 15

Figure 13. The Chemical Structure of Diphenylmesitylborano (24) . . . . . 15

Figure 14. Attempted Synthesis of Diphenyl(2,4,6-tri-tert-butylphenyl)borane (26) 16

Figure 15. Successful Synthesis of Diphenyl(2,4,6-triisopropylphenyl)borane (28) . 16

Figure 16. GC/MS Ion Chromatograms of Dechlorination of Chlorobenzene by $\mathrm{NaNAPH} . \ldots \ldots \ldots \ldots 19$

Figure 17. GC/MS Ion Chromatograms of Dechlorination of 4-Chlorobiphenyl

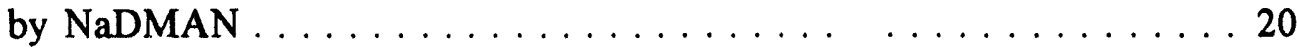

Figure 18. GC/MS Ion Chromatograms of Aroclors 1242 and $1254 \ldots \ldots 21$ 
Figure 19. GC/MS Ion Chromatograms of Dechlorination of Aroclor 1242

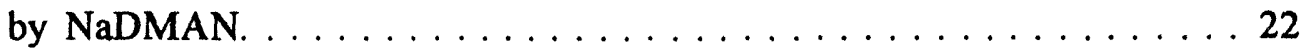

Figure 20. GC/MS Ion Chromatograms of Dechlorination of Aroclor 1254

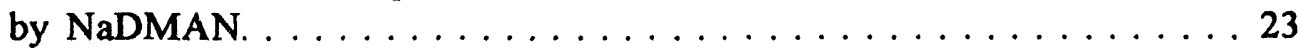

Figure 21. The ${ }^{1} \mathrm{H}$ NMR Spectrum $\left(\mathrm{CDCl}_{3}, 270 \mathrm{MHz}\right)$ of $22 \ldots \ldots \ldots \ldots 25$

Figure 22. The ' $\mathrm{H}$ NMR Spectrum $\left(\mathrm{CDCl}_{3}, 270 \mathrm{MHz}\right)$ of

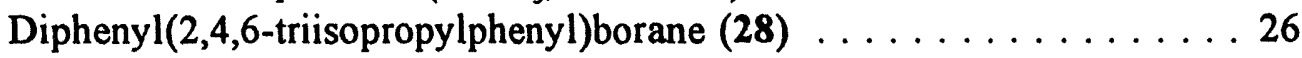

Figure 22. The GC/MS Ion Chromatograms of

Diphenyl(2,4,6-triisopropylphenyl)borane (28) $\ldots \ldots \ldots \ldots \ldots 27$ 


\section{LIST OF TABLES}

Table

Page No.

Table 1. Detection Limits of Chlorinated Aromatics by the GC/MS System . . . 17 


\section{EXECUTIVE SUMMARY}

It is the objective of this research to evaluate the existing technologies for destruction of polychlorinated biphenyls (PCBs) by chemical methods. We will also focus on establishing chemical treatment procedures for dechlorination of PCBs. We will probe into the detailed reaction mechanisms of various dechlorination processes so as to gain fundamental understanding of the reaction pathway. Such information will provide insight in designing an efficient and economical system.

In the first part of this report, the main focus will be on the assessment of the current technologies and the discussion of the basic chemical reactions behind these treatment procedures. Three commercial vendors listed with the Environmental Protection Agency (EPA) for their involvement in chemical dechlorination of PCBs have been contacted: (1) Trinity Environmental Technologies, Inc., (2) Chemical Waste Management, Inc., and (3) Roy F. Weston, Inc.

The Trinity Environmental Technologies, Inc. utilizes sodium metal to dechlorinate PCBs to inert biphenyl and sodium chloride. A general discussion of dechlorination of PCBs by sodium and other alkali metals is included in this report.

The Chemical Waste Management, Inc. utilizes a nucleophile-based process (the KGME process) that enables the destruction of halogenated aromatic compounds through the successive replacement of one or more halogen atoms with 2-methoxyethoxy moiety. A demonstration of this process was given at the ReSolve Superfund site at North Dartmouth, Massachusetts in the summer of 1992 under the sanction of EPA. Destruction of PCBs by nucleophilic displacement of chlorine atoms with a strong base is also reviewed in this report.

The procedure employed by Roy F. Weston, Inc. involves the use of zinc and a mild organic acid to generate nascent (atomic) hydrogen, which replaces aromatic halogen atoms in toxic organics in the presence of gentle heat and an unspecified catalyst. This process has been tested only in the laboratory in gram quantity and has not been scaled up to pilot plant level. Other catalytic dechlorination processes are also discussed in this report.

The direct photodegradation of PCBs with UV or sunlight irradiation proceeds with low efficiency. However, the efficiency of photodegradation can be dramatically enhanced with appropriate photo-sensitizers and other additives. The basic reaction mechanism of the photodegradation process is discussed in this report. 
In the second part of this report, experimental results obtained from dechlorination of aromatic halides, including chlorobenzene, 4-chlorobiphenyl, Aroclor 1242, and Aroclor 1254, by sodium 1-dimethylaminonaphthalide (NaDMAN) will be discussed. The easy recovery of 1dimethylaminonaphthalene from the reaction mixture offers a significant advantage for its use as an electron carrier for sodium metal during the dechlorination process. However, as a reagent NaDMAN in tetrahydrofuran is not very stable. It is necessary to prepare NaDMAN at $-10^{\circ} \mathrm{C}$ and immediately prior to use. Storing NaDMAN solution at room temperature resulted in gradual loss of its reactivity over several days.

In the third part of this report, research efforts toward developing sterically hindered and chemically stable triarylboranes as potential electron carriers for sodium metal will be discussed. Because of the presence of an empty p-orbital on the boron atom which allows an electron from sodium metal to reside over it, triarylboranes are excellent electron carriers. Moreover the corresponding radical anions are stable over an extended period of time at room temperature, allowing dechlorination proccdure to be carried out at ambient temperature. Attempts are being made to try to incorporate an amino group into the chemical structure of sterically hindered triarylboranes with the intention for easy recovery after the dechlorination process is complete. Attaching a suitable electron carrier to a polymer backbone to allow easy separation is also being investigated. 


\section{INTRODUCTION}

\subsection{Background}

Polychlorinated biphenyls, commonly referred to as PCBs, are one of the major organic pollutants in the environment. The chemical structures of three representative polychlorinated biphenyls are shown in Figure 1. The large scale use of PCBs for a variety of purposes, especially as a dielectric material in capacitors and transformers as well as plasticizers and solvents in plastics and printing inks, coupled with the chemical stability of PCBs against degradation have resulted in their worldwide accumulation in the environment.

Figure 1. Chemical Structures of Three Representative Polychlorinated Biphenyls
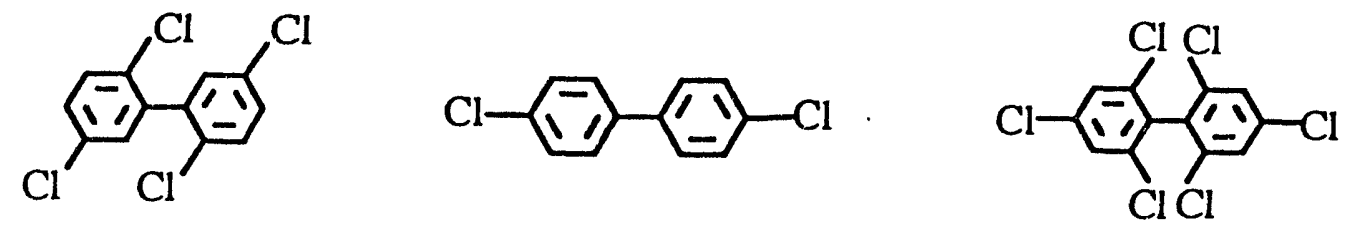

The total worldwide production of PCBs through 1980 is estimated to be approximately 2.4 billion pounds. Of the 1.25 billion pounds of PCBs estimated to have been produced in the United States, mainly by Monsanto under the registered trademark of Aroclor, about 24 million pounds are believed to have been released to the environment.

The toxic effects of PCBs have been well documented. One of the most famous cases occurred in Japan in 1968 when PCBs from a defective heat exchanger were leaked into rice oil which was then consumed by more than 1000 people.' For those who consumed more than 0.5 grams (average consumption was 2 grams), severe acne, darkened skin, and eye damage developed. Recovery was slow and these symptoms were still present even after three years. Numerous studies have also reported the toxic effort of PCBs to a wide range of wildlife, including mink, some species of shellfish, shrimp, and fish, and especially those birds that are at the top of the food chain, such as eagles, hawks, falcons, and pelicans. ${ }^{2}$ The chronic (longterm) toxicity to humans and other species is yet to be fully realized.

\subsection{Destruction of PCBs}

Because of the thermodynamic stability of PCBs, destruction by incineration and other chemical degradation processes are difficult. Disposal of untreated PCBs by landfill is virtually banned by the Resource Conservation and Recovery Act (RCRA). Currently, incineration is still the most widely used method for treating many hazardous wastes, including PCBs. Incinerators are strictly regulated to assure effective destruction of PCBs. For example, the regulation requires $99.9999 \%$ (six-9's) efficiency for the degradation of nonliquid PCBs by incineration. However, despite such strict regulations it is possible that trace amounts of PCBs as well as other combustion byproducts, including the much more toxic polychlorinated benzofurans and dioxins, could still be released to the environment through stack emission. Concerns about the health impact of PCBs, dioxins, and other organic compounds formed and emitted into the environment 
have on surrounding communities have created many controversies and strong opposition from various environmental groups. It is now typical to take more than ten years and prolonged legal battle before an incinerator for industrial hazardous wastes could be constructed and operated. One recent example involves the Waste Technologies Industries (WTI) facility in East Liverpool, Ohio which will soon begin its test burn only after 13 years of planning and construction, more then 20 court cases, and even the intervention of the Vice President of the United States. In a recent article of the Chemical and Engineering News, many issues concerning hazardous waste incineration were raised and discussed. ${ }^{3}$ It is clear that there is a need to continue research and development of alternative chemical processes for treating hazardous wastes in general and PCBs and other chlorinated aromatic compounds in particular.

\subsection{Purpose and Scope of Report}

It is the purpose of this report to first evaluate the existing technologies for the destruction of PCBs by chemical methods. We will focus mainly on the current commercial processes as well as procedures recently published in the literature, including dechlorination of PCBs by sodium and other alkali metals, by the use of strong base, by catalytic dechlorination, and by photochemical degradation. The basic chemical reactions behind these dechlorination processes will also be discussed. Experimental results obtained from dechlorination of aromatic chlorides by sodium 1-dimethylaminonaphthalide will also be discussed. Research efforts toward synthesizing sterically hindered and chemically stable triarylboranes as electron carriers for sodium metal are included in the report.

\section{LITERATURE REVIEW OF CHEMICAL DESTRUCTION OF PCBS}

Development of chemical process for the destruction of PCBs and other chlorinated aromatic compounds continues to be a research area of intense interest. Limited success has been achieved in some specific cases. Excellent review articles of the chemical processes are available in the literature. ${ }^{4}$ In addition, a recent monograph by Mitchell D. Erickson covered broad issues concerning PCBs, especially the analytical procedures. ${ }^{5}$ In this report, four general methods for chemical destruction of PCBs will be reviewed.

\subsection{Dechlorination of PCBs by Sodium and Other Alkali Metals}

The Goodyear Tire and Rubber Company patented a sodium-naphthalide process for PCB destruction in 1981 and later released it for public use. ${ }^{6}$ This process removes chlorine atoms from the PCBs and combines them with sodium to form sodium chloride (table salt).

The use of sodium metal for the dechlorination of aromatic compounds is well established in the literature. ${ }^{7}$ The reaction mechanism is believed to involve first transferring an electron from sodium to the chlorinated aromatic compound, such as chlorobenzene (1) to form the corresponding radical anion, such as (2) (Fig. 2). The radical anion 2 then loses a chloride ion to form phenyl radical 3, which then acquires an electron from sodium to form phenyl anion 4. Subsequent quenching of 4 with water affords the dechlorinated adduct as benzene (5). In the 
case of PCBs, such reaction cycle is repeated several times until all the chlorine atoms are removed. The resulting anions then are quenched with water.

Figure 2. Dechlorination of Chlorobenzene by Sodium

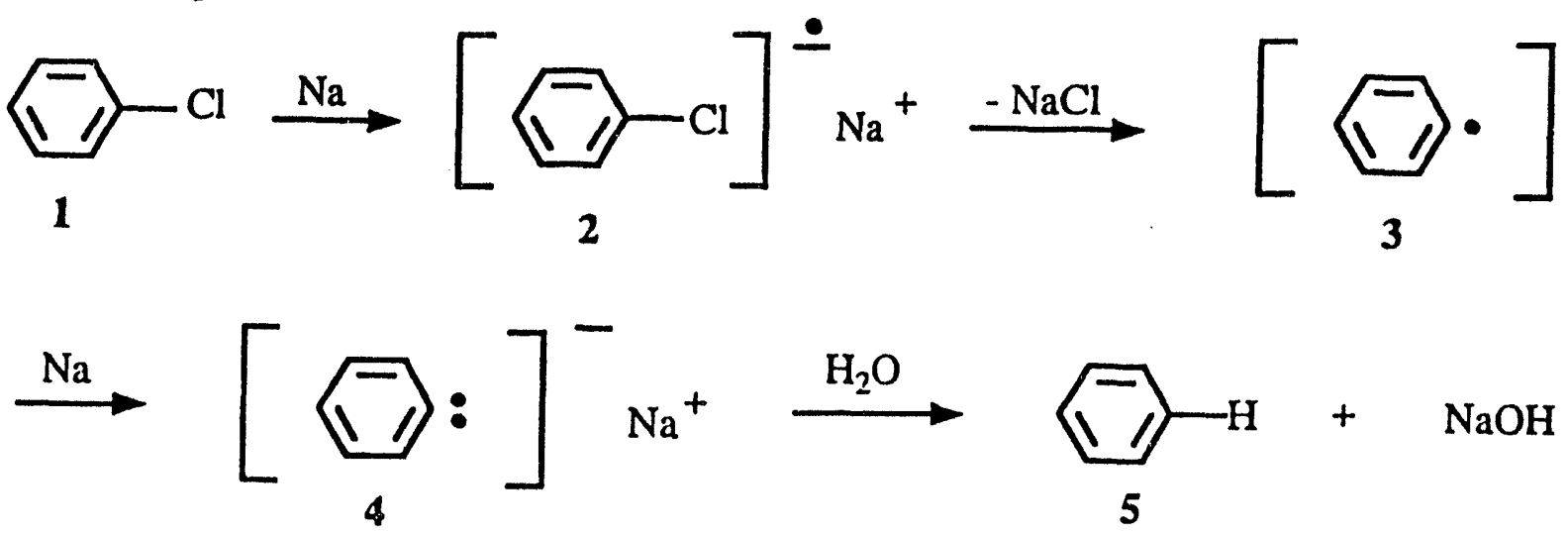

Because sodium metal is not very soluble in organic solvents, the Goodyear process uses naphthalene as an electron carrier to improve the efficiency of electron transfer. The process normally takes 2 hours at $60^{\circ} \mathrm{C}$ for complete dechlorination. However, to effectively remove low levels of PCBs (ca. 100 parts per million, ppm) from contaminated oil down to ca. $3 \mathrm{ppm}$, far lower than the requirement set by the Environmental Protection Agency (EPA), a large excess of the reagent is required. Typically, the reagent to chloride molar ratio must be from 25 to 500 to obtain significant reduction of the PCB concentration. Quenching the excess sodiumnaphthalide reagent with water must be conducted slowly and with extreme caution. This is because generation of hydrogen gas will occur if sodium is contacted with water, creating a potentially dangerous situation for explosion.

In 1984, Goodyear disclosed an improved process with the elimination of the water quench step. $^{8}$ The difficulties and disadvantages associated with a water quench of the alkali metal aromatic radical anion reagent are overcome by utilizing carbon dioxide $\left(\mathrm{CO}_{2}\right)$ as the excess reagent quenching material. No hydrogen gas is involved and at no time does water enter the system. The exclusion of water from the process allows for the recovery of the reaction solvent, such as tetrahydrofuran, in pure, dry form, eliminating additional process step, does not generate a waste water stream for disposal, and improves overall process safety.

The Goodyear process uses naphthalene, which has been classified as a priority pollutant by EPA, and its use is restricted. A number of processes have since been developed, utilizing other compounds as substitute for naphthalene. ${ }^{9-11}$ The Sunohio, Inc. of Canton, Ohio developed proprietary compounds to replace naphthalene in a sodium-based process for reclamation of transformer oils containing PCBs. The Sunohio procedure was reviewed preciously. ${ }^{9}$

In a recently patented procedure, ammonium salt was used to accelerate the reductive cycle of dechlorination and to serve as a proton source to hydrogenate and quench the dehalogenated polyhaloaromatics. ${ }^{10}$ It was also reported in a separate patented procedure that 
liquid hydrosiloxane could facilitate the reductive dechlorination so that it will proceed to completion at room temperature in relatively short times." It was claimed that PCBs are dechlorinated to biphenyl and its higher molecular weight oligomers with greater than $99 \%$ efficiency.

Currently, the sodium-based process is employed by the Trinity Environmental Technulogies, Inc., using a modified version of the Goodyear process. It is claimed that this process is cost competitive compared to incineration for the treatment of PCB oils. However, this process will not be able to treat PCB contaminated soil. A block diagram of the Trinity process is given in Figure 3.

\subsection{Destruction of PCBs by the Use of Strong Base}

The use of strong base to attack PCBs has been investigated. The KPEG process, which uses potassium metal $(\mathrm{K})$ and polyethyleneglycol $(\mathrm{PEG})$ to destroy $\mathrm{PCBs}$, is an example of such a treatment procedure. ${ }^{4 a}$ Potassium metal reacts with polyethyleneglycol, such as diethyleneglycol (6), to form the correspond alkoxide (7), a strong base, wt ich than attacks PCBs by nucleophilic displacement (Fig. 4).

Figure 4. Destruction of PCBs by the KPEG Process

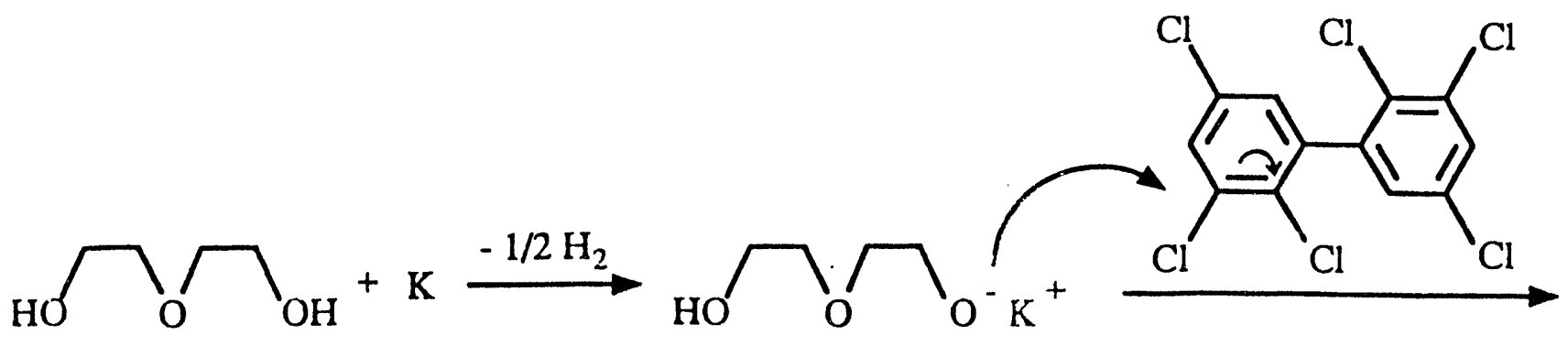
6

7
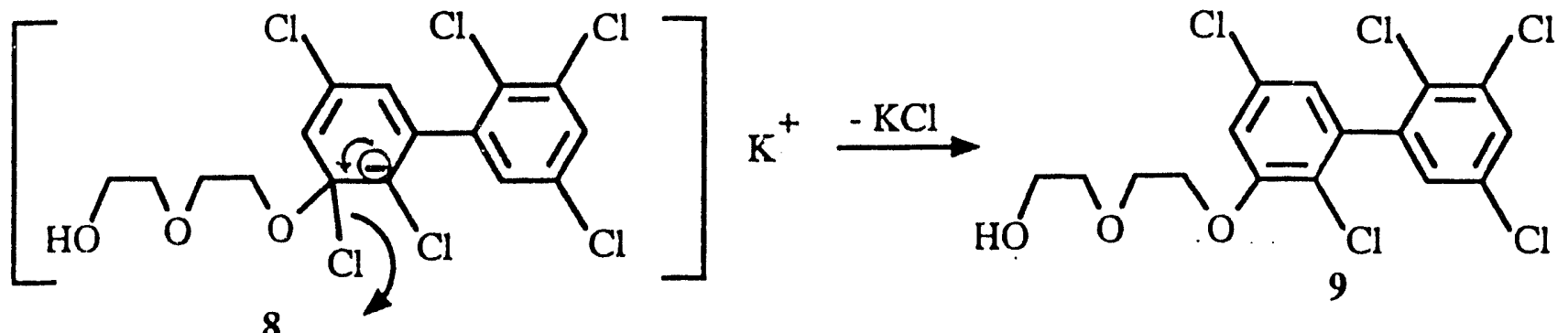<smiles>[Z]Oc1cc(OC)c(OC)c(-c2cc(OC)cc(OC)c2OC)c1</smiles> 
Figure 3. Block Diagram of the Trinity PCB Treatment Process

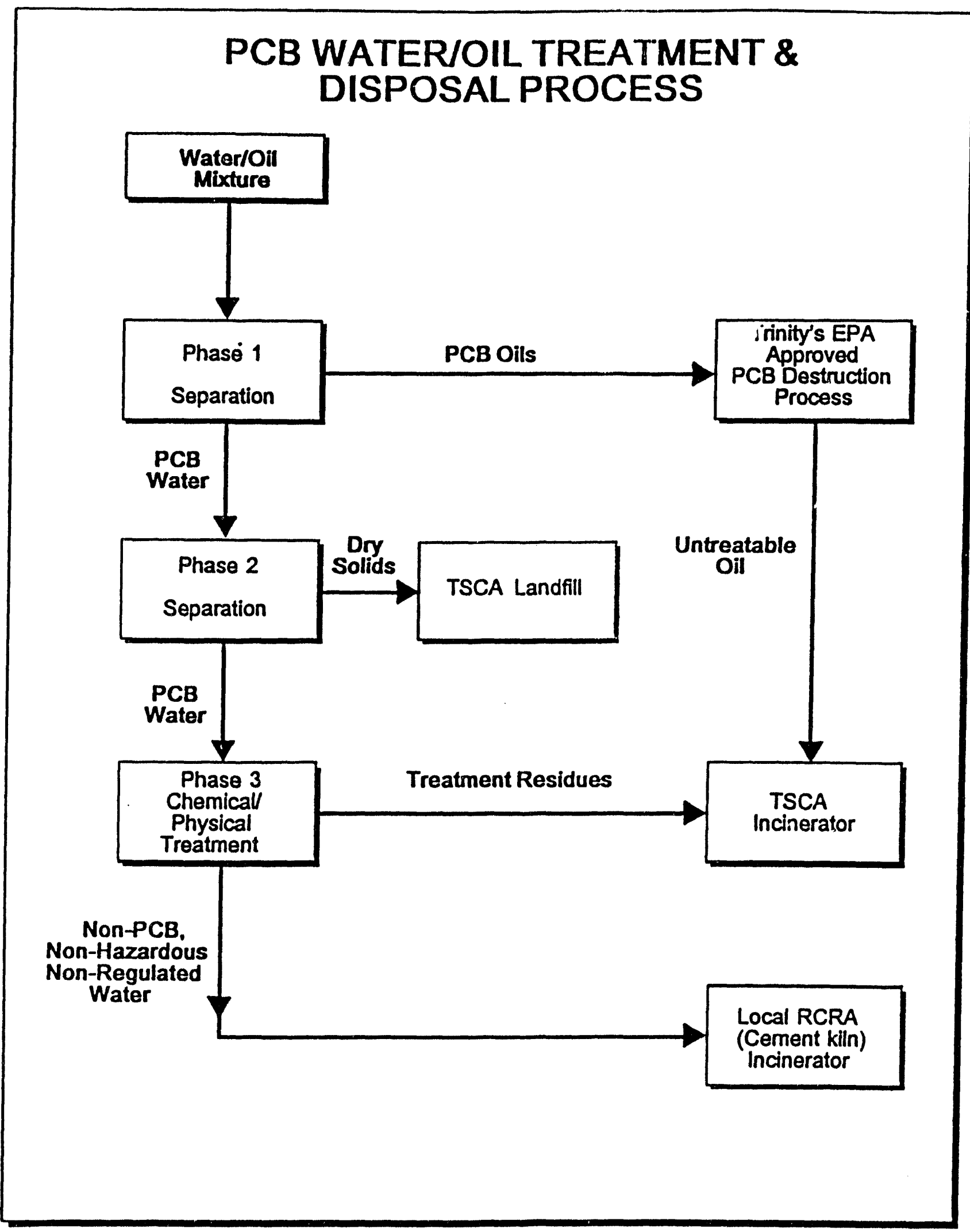


Although the exact reaction mechanism of the KPEG process probably has not been fully established. It can be speculated that for the highly chlorinated biphenyls, the electron deficient benzene ring could be attacked by 7 to form 8 . Subsequent loss of a chloride ion from 8 could lead to 9 with the net effect of replacing a chlorine atom with a polyethyleneglycol. After successive replacement of chlorine atoms with polyethyleneglycol, complete dechlorination to form polyhydroxylated biphenyls $\mathbf{1 0}$ could thus be achieved.

It is also possible that a competing reaction pathway involving the formation of a benzyne intermediate 11 may also be responsible for the dechlorination process (Fig. 5). ${ }^{12}$

Figure 5. The KPEG Process via the Benzyne Pathway
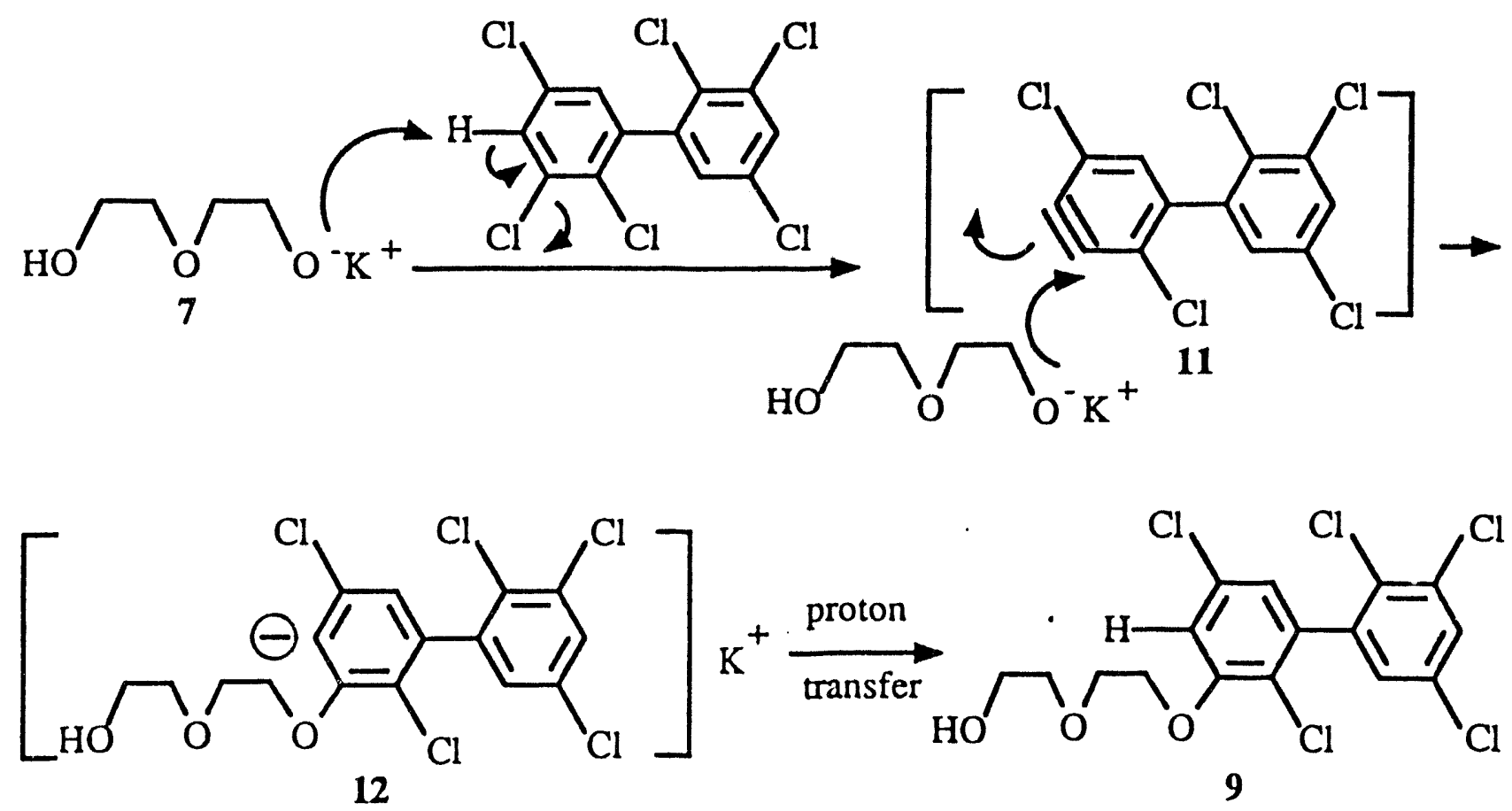

Initial dehydrochlorination of PCBs to form benzyne 11 followed by the attack of 11 with an alkoxide would lead to 12 . Subsequent proton transfer could also result in the displacement of a chlorine atom by a polyethyleneglycol as shown in 9 .

Currently, a modified KPEG process is being utilized by the Chemical Waste Management, Inc. for the destruction of PCBs. The reagent, KGME (14), is generated in situ via the addition of $\mathrm{KOH}$ to 2-methoxyethanol (glycol methyl ether, GME, 13), followed by azeotropic removal of water (Fig. 6). The use of $\mathrm{KOH}$ instead of potassium metal as in the KPEG process is an improvement which avoids the generation of hazardous hydrogen gas during reagent preparation. 
Figure 6. Preparation of the KGME Reagent

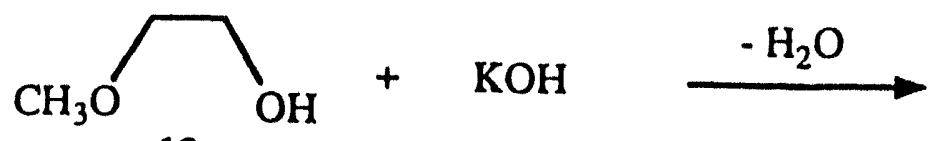

13

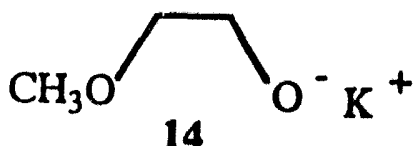

A demonstration of the KGME process was conducted at the ReSolve Superfund sit at North Dartmouth, Massachusetts in the summer of 1992 under the sanction of EPA. Although a final report on the demonstration is not available at the present time, a private communication with Dr. Richard J. Ayen, Vice President and General Manager of the Chemical Waste Management, Inc., indicates that the KGME process is more expensive to operate than thermal desorption of PCB contaminated soil followed by off-site incineration of the resulting oil.

In early 1991 , reports started to appear in the press indicating that quicklime, or calcium oxide $(\mathrm{CaO})$, could destroy PCBs. $^{13}$ A success in such a process could mean a low cost remediation of $\mathrm{PCB}$ pollution. However, many researchers were skeptical of those reports because the basic chemical reaction between quicklime and water generates mainly $\mathrm{Ca}(\mathrm{OH})_{2}$, which is a source of hydroxide ion. Hydroxide ion had not been shown to effectively destroy PCBs. Preliminary results from an independent study by the EPA's Risk Reduction Engineering Laboratory in Cincinnati suggest that the reduction of PCB content is mostly attributable to volatization and is not due to actual PCB destruction. ${ }^{13,14}$

\subsection{Catalytic Dechlorination of PCBs}

The catalytic dechlorination of PCBs at $180^{\circ} \mathrm{C}$ to biphenyl with $5 \%$ platinum or palladium on 60/80 mesh glass beads over hydrogen gas was reported by Berg et al. in 1972. ${ }^{15}$ The use of $69 \%$ nickel on kieselguhr in the presence of sodium hydroxide and 50 atm of hydrogen gas at $115^{\circ} \mathrm{C}$ for 6 hours also effectively dechlorinated Aroclor $1248 . .^{16}$ It was later discovered that sodium borohydride could replace hydrogen gas as the reducing agent. ${ }^{17}$ One example of the study showed that $0.3 \mathrm{mmol}$ of Aroclor 1254 could be most effectively reduced to biphenyl $(97 \%)$ by treating with $2.0 \mathrm{mmol}$ of $\mathrm{NiCl}_{2}$ and $60 \mathrm{mmol}$ of sodium borohydride in 2-propanol at ambient temperature and pressure. The disadvantage of this process is that a rather large ratio of sodium borohydride to PCBs is required to reach complete dechlorination.

In a process patented by the Union Carbide Corporation in $1983,{ }^{18} 100 \mathrm{mg}$ of a commercial Aroclor in $1 \mathrm{~mL}$ of methanol was treated with $0.03 \mathrm{~g}$ of $\mathrm{NiCl}_{2}, 0.5 \mathrm{~g}$ triphenylphosphine, $0.25 \mathrm{~g} \mathrm{NaI}$ and $1.0 \mathrm{~g}$ of zinc dust in $10 \mathrm{~mL}$ of wet $N, N$-dimethyl formamide (DMF) at $60^{\circ} \mathrm{C}$ for 4 hours. The zinc dust in the presence of sodium iodide serves as the reducing means to keep the nickel in a zero valance state for effective catalytic dechlorination. 
Currently, the Roy F. Weston, Inc. uses a catalytic dehydrochlorination procedure to remediate soils, sludges, and sediments contaminated with PCBs. The process uses zinc and a mild organic acid to generate nascent (atomic) hydrogen, which replaces aromatic halogen atoms in toxic organics in the presence of gentle heat and an unspecified catalyst. However, this process has been tested only in the laboratory in gram quantity and has not been scaled up to pilot plant level.

\subsection{Photochemical Dechlorination of PCBs}

The use of photochemical methods for the destruction of PCBs have received considerable attention. The direct photodegradation of PCBs with UV or sunlight irradiation proceeds with low efficiency. This is because once the heavier chlorinated and more photo-sensitive biphenyls are depleted, the dechlorination process becomes very slow. Typically, only about $25 \%$ of Aroclor 1254 and $10 \%$ of Aroclor 1260 are reacted after 10 hours. ${ }^{199}$ However, the efficiency of photodegradation has been shown to be dramatically enhanced with appropriate photosensitizers and other additives. For example, it was reported that amines, ${ }^{20}$ borohydrides, ${ }^{19}$ alkaline alcohols, ${ }^{21}$ and hydroquinones ${ }^{22}$ greatly enhanced the rate of photodechlorination of PCBs in solution.

Recently, the use of acetone as a photosensitizing agent in alkaline 2-propanol has been shown to be particularly promising, allowing dechlorination of Aroclor 1254 at wavelengths compatible with those available from the sun. ${ }^{23}$ Total disappearance of Aroclor 1254 and the formation of biphenyl occurred in less than 25 minutes.

A free radical chain reaction has been proposed to be the pathway of the photodechlorination process (Fig. 7). The acetone molecule is excited by irradiation to a high energy triplet state, $T_{1}\left(n, \pi^{*}\right)$, which then abstracts a hydrogen atom from 2-propanol to give the ketyl radical 15. The ketyl radical then loses a proton to the alkaline medium, producing the ketyl radical anion 16. The Aroclor in turn reacts with the ketyl radical anion through an electron-transfer process given unstable aryl radical anion 17 which releases a chloride anion, producing the aryl radical 18. The aryl radical then abstracts a hydrogen atom from 2-propanol to furnish dechlorinated biphenyls 19 and the ketyl radical 15, allowing the propagation cycle for dechlorination of PCBs to continue until all of the chlorine atoms are removed.

The acetone-induced photodegradation of PCBs is a very attractive method because of the low costs of the reagents. Unfortunately, under similar conditions photodechlorination of extrac's of Aroclor 1254 contaminated soil proceeded with low efficiency. Clearly, continued research is needed to bring the photodegradation method to practical use. 
Figure 7. Acetone-Induced Photochemical Degradation of PCBs

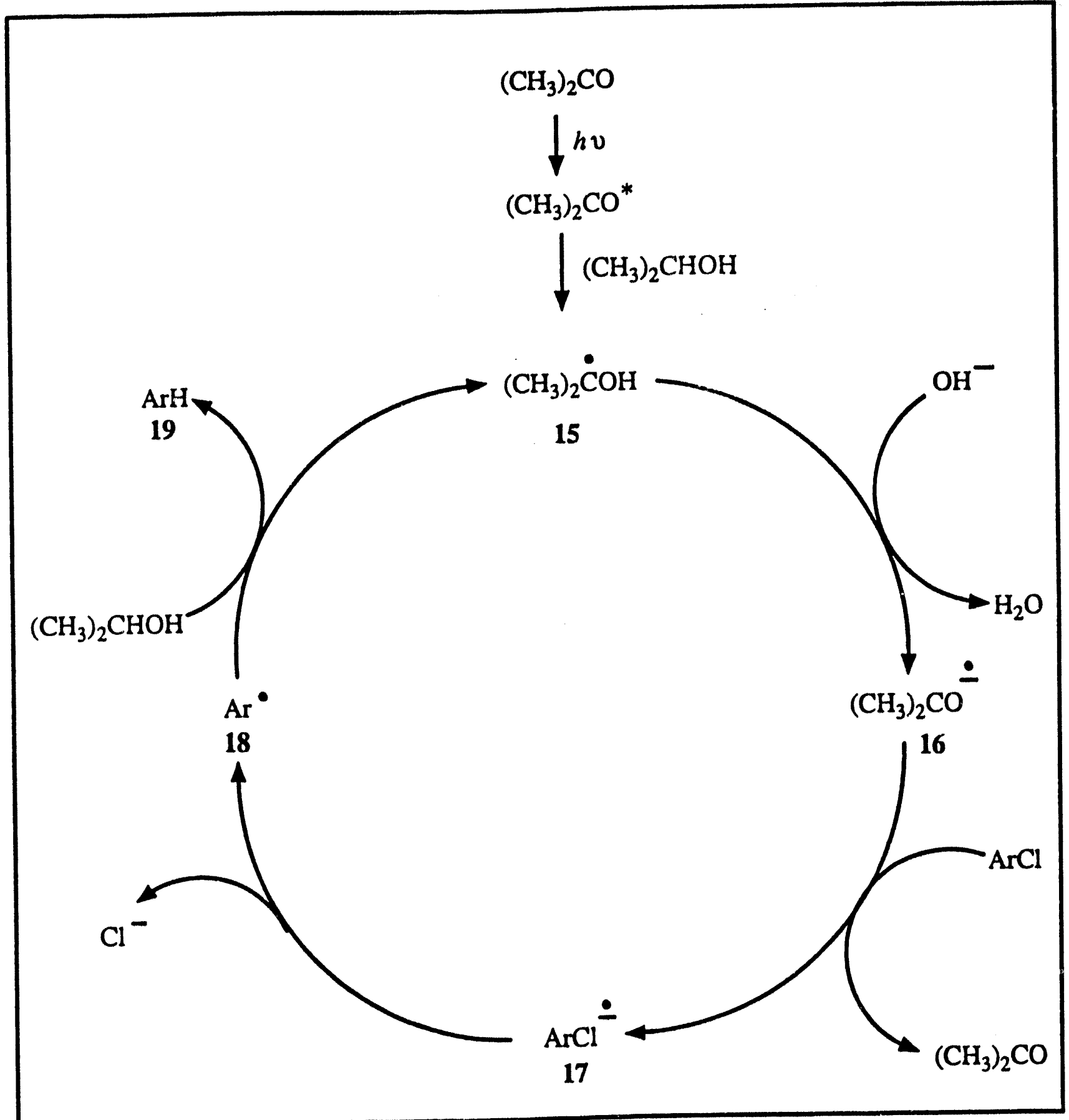




\section{CHEMICAL DESTRUCTION OF CHLORINATED AROMATICS BY SODIUM 1-DIMETHYLAMINONAPHTHALIDE (NaDMAN)}

The use of sodium metal in the presence of an appropriate electron carrier is potentially a good process for destruction of PCBs. We have selected this area to conduct our initial investigation because the process is simple and easy to carry out in the laboratory. It also provides an opportunity to establish an analytical procedure to determine the efficiency of the process for destruction of PCBs by sodium metal-based method and other processes in the subsequent studies.

We have first repeated the Goodyear process by using sodium metal in the presence of naphthalene as an electron carrier for PCB destruction. Chlorobenzene was used as a surrogate for PCBs and sodium naphthalide (NaNAPH) was prepared according to the published procedure. ${ }^{6}$ Indeed the dechlorination process is very facile at room temperature. The reaction was followed by periodically withdrawing a small sample from the reaction mixture and quenching it with water. The extent of dechlorination was then analyzed by using a GC/MS. It was found that the reaction was essentially complete within a few minutes with reduction of chlorobenzene concentration from $595 \mathrm{ppm}$ by weight in tetrahydrofuran to less than $1 \mathrm{ppm}$ (Figure 8).

Figure 8. Dechlorination of Chlorobenzene by NaNAPH

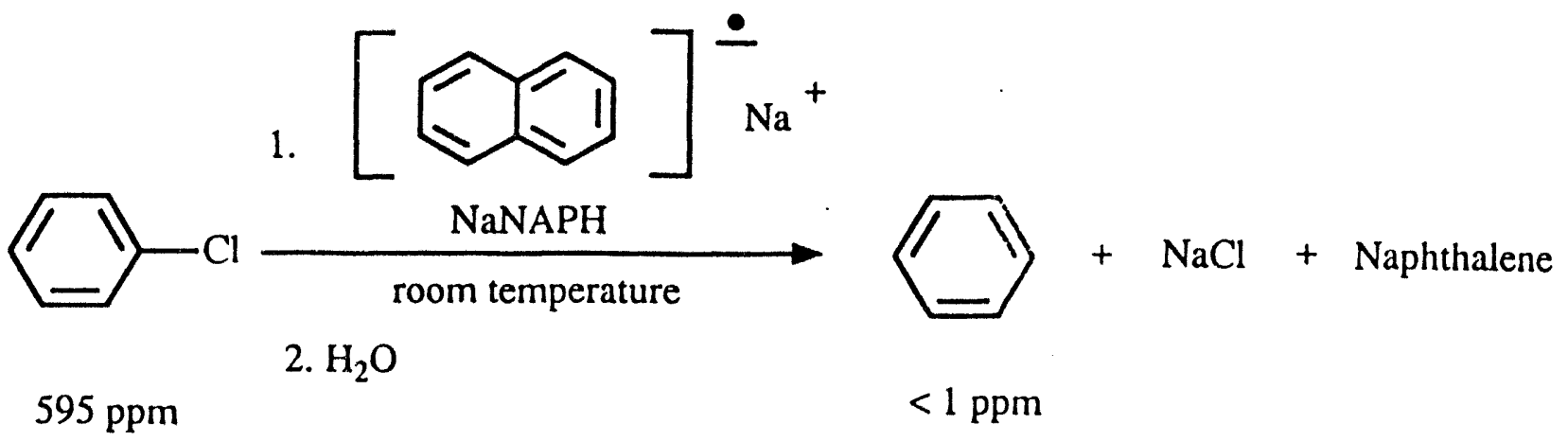

With the establishment of the NaNAPH dechlorination condition as a baseline for comparison and a GC/MS method for following the reaction, we then turned our attention to finding an alternative electron carrier as a substitute for naphthalene. As indicated earlier, naphthalene has been classified as a priority pollutant by EPA, and its use is restricted. It is also difficult to separate naphthalene from other organic products after the dechlorination process is complete. A careful search of literature brought to our attention the use of 1dimethylaminonaphthalene as a substitute for naphthalene. ${ }^{24}$ Because of the presence of an amino functionality, l-dimethylaminonaphthalene is very soluble in aqueous acidic solution and can be easily separated from other organic compounds. It can be regenerated by neutralizing the aqueous solution for easy recovery and reuse, minimizing pollution to the environment and reducing the cost of the dechlorination process. 
Although the use of sodium 1-dimethylaminonaphthalide (NaDMAN) for dechlorination of aliphatic chlorides was reported, ${ }^{24}$ extending the use of this reagent to aromatic chlorides had not been studied. It was gratifying to observe that sodium 1-dimethylaminonaphthalide was also very efficient in dechlorinating aromatic chlorides, such as chlorobenzene, 4-chlorobiphenyl, Aroclor 1242, and Aroclor 1254 (Figure 9). The efficiency of dechlorination is similar to that of NaNAPH. Recovery of 1-dimethylaminonaphthalene after the reaction was essentially quantitative.

Figure 9. Dechlorination of Aromatic Chlorides by NaDMAN

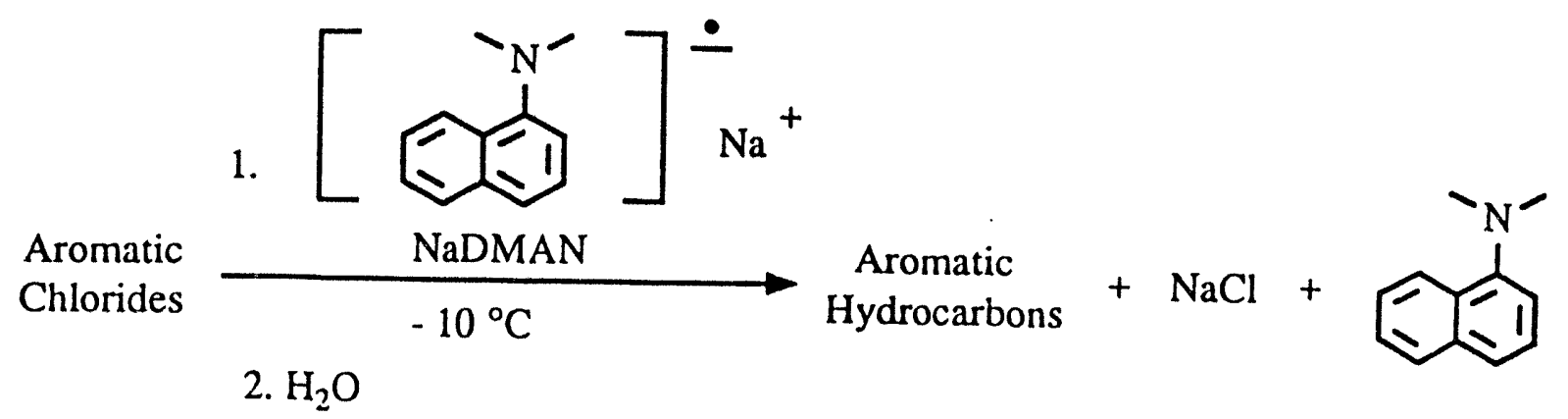

In comparison with naphthalene, the easy recovery of 1-dimethylaminonaphthalene from the reaction mixture offers a significant advantage. However, as a reagent NaDMAN in THF is less stable than $\mathrm{NaNAPH}$. It was necessary to prepare $\mathrm{NaDMAN}$ at lower temperature $\left(-10^{\circ} \mathrm{C}\right)$ and immediately prior to use. Storing NaDMAN solution at room temperature resulted in gradual loss of its reactivity over several days.

\section{SYNTHESIS OF STERICALLY HINDERED TRIARYLBORANES AS POTENTIAL ELECTRON CARRIERS FOR SODIUM METAL}

It has been known for sometime that triarylboranes are excellent electron carriers because of the presence of an empty p-orbital on the boron atom, allowing an electron from sodium metal to reside over it. ${ }^{25}$ One such example is trimesitylborane (20), which reacts readily with sodium to form the corresponding radical anion 21 (Fig. 10). ${ }^{25}$ Radical anion 21 is stable over an extended period of time at room temperature and dechlorinates 4,4'-dichlorobiphenyl almost instantaneously. $^{26}$ Because of severe steric hinderance surrounding the boron atom, the regenerated trimesitylborane is stable to water, making it possible to workup the reaction mixture with water. 
Figure 10. Reaction of Trimesitylborane (20) with Sodium Metal<smiles>Cc1cc(C)c(B(c2c(C)cc(C)cc2C)c2c(C)cc(C)cc2C)c(C)c1</smiles>

20

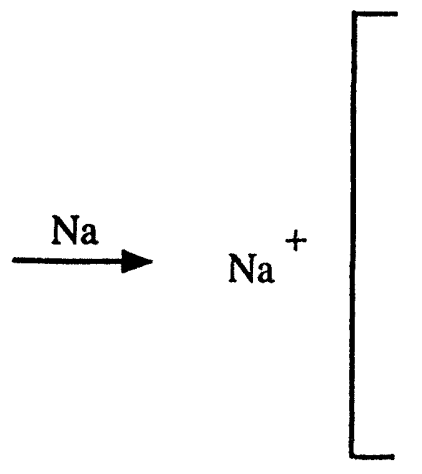

21

We envisioned that a sterically hindered triarylborane substituted with a dimethylamino group, such as 4-(dimethylamino)phenyldimesitylborane $(22)^{27}$ in Figure 11 , could serve as an excellent electron carrier. The anticipated higher stability of the corresponding radical anion 23 over that of NaDMAN coupled with easy recovery by a simple acid extraction could make the dechlorination process operationally feasible.

Figure 11. 4-(Dimethylamino)phenyldimesitylborane (22) as an Electron Carrier<smiles>Cc1cc(C)c(B(c2ccc(N(C)C)cc2)c2c(C)cc(C)cc2C)c(C)c1</smiles>

22

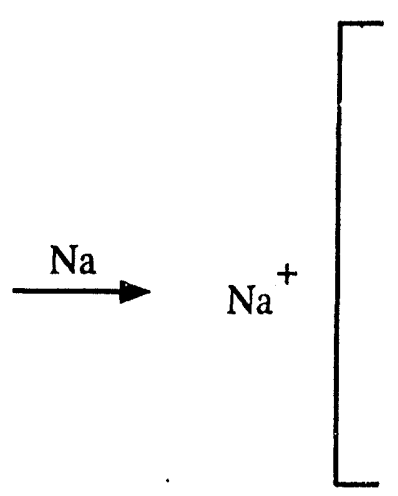

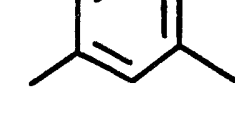

23

Recently, it came to our attention that 4-(dimethylamino)phenyldimesitylborane (22) was reported to be stable toward water and was easily prepared by using the protocol outlined in Figure $12 .{ }^{27}$ We have prepared 22 by using a modified procedure in order to achieve higher yield (72\%). The ability of 22 in serving as an electron carrier for sodium metal for dechlorination and the feasibility of recovering $\mathbf{2 2}$ from the reaction mixture for reuse were also investigated. 
Figure 12. Synthetic Procedure for 4-(Dimethylamino)phenyldimesitylborane (22)
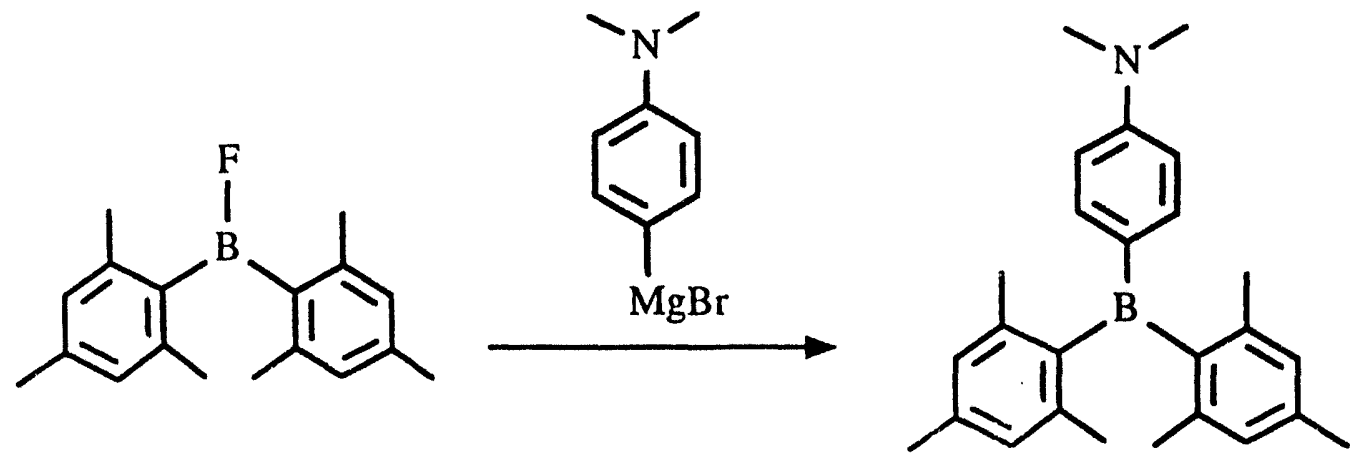

$22,58 \%$ yield

To our disappointment, it was discovered that borane radical anion 23 failed to exhibit efficient dechlorination property toward chlorobenzene. It appeared that a large excess of 23 (approximately 10 times) was required to achieve about $30 \%$ dechlorination. The percentage of the recovered 22 after the dechlorination reaction was equally disappointing (50\%). The GC/MS ion chromatogram indicated the presence of substantial amounts of mesitylene and $N, N$ dimethylaniline, hydrolysis products of 22 .

We have also spent considerable efforts in trying to determine the extent of steric hinderance surrounding the boron atom that will be required in order to prevent hydrolysis of triarylborane by water. This information is of crucial importance because hydrolytic workup will be performed after the dechlorination process is complete. It is known in the literature that the sterically unhindered triphenylborane is readily hydrolyzed by water, whereas trimesitylborane is stable to water. ${ }^{28}$ We also quickly learned in our laboratory that diphenylmesitylborane (24) in Figure 13 containing only one modestly congested aryl group is also very susceptible to water hydrolysis.

Figure 13. The Chemical Structure of Diphenylmesitylborane (24)<smiles>Cc1cc(C)c(B(c2ccccc2)c2ccccc2)c(C)c1</smiles> 
Repeated attempts to synthesize diphenyl(2,4,6-tri-tert-butylphenyl)borane (26) having an extremely congested aryl group by treating methyl diphenylborinate $(25)^{29}$ with $2,4,6$-tri-tertbutylphenyllithium $^{30}$ as shown in Figure 14 were unsuccessful. Perhaps 2,4,6-tri-tertbutylphenyllithium with two very bulky tert-butyl groups at the ortho positions is too hindered for the reaction to take place.

Figure 14. Attempted Synthesis of Diphenyl(2,4,6-tri-tert-butylphenyl)borane (26)<smiles>COB(c1ccccc1)c1ccccc1</smiles>

25

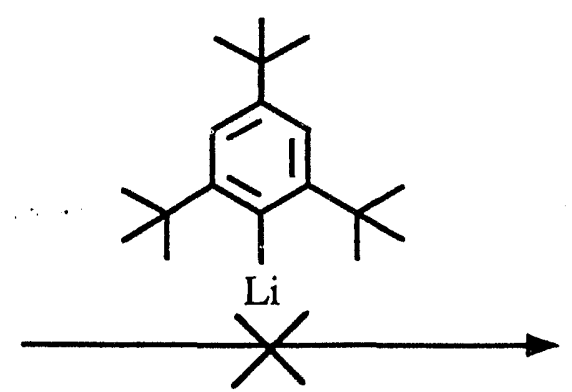

$\mathrm{Li}$<smiles>Cc1c(C(C)(C)C)cc(C(C)(C)C)cc1C(C)(C)C</smiles><smiles>B1Cc2ccccc21</smiles>

26

Fortunately, by using a slightly less hindered 2,4,6-triisopropyllithium (27), ${ }^{31}$ the resulting diphenyl(2,4,6-triisopropylphenyl)borane (28) can be easily synthesized as shown in Figure 15 . Triarylborane 28, a new triarylborane which had not been synthesized before, exhibited high stability toward water and air, making it possible to conduct hydrolytic workup after the dechlorination process.

Figure 15. Successful Synthesis of Diphenyl(2,4,6-triisopropylphenyl)borane (28)<smiles>COB(c1ccccc1)c1ccccc1</smiles>

25<smiles>[Z1]c1c(C(C)C)cc(C(C)C)cc1C(C)C</smiles>

27<smiles>CC(C)c1cc(C(C)C)c(B(c2ccccc2)c2ccccc2)c(C(C)C)c1</smiles>

$28,39 \%$ yield 


\section{EXPERIMENTAL SECTION}

\subsection{Establishment of an Analytical Procedure for Chlorinated Aromatics and PCBs}

A GC/MS system comprised of an HP 5890A Gas Chromatograph and an HP 5970B Mass Selective Detector was employed as the analytical tool for identification of organic halides and quantitative determination of their concentrations. A flexible fused silica capillary column (HP-1, crosslinked methyl silicone gum, $25 \mathrm{~m} \times 0.20 \mathrm{~mm}$ ID $\times 0.33 \mu \mathrm{m}$ thickness) was installed in the GC oven. The GC conditions for analytical studies are summarized as follows:

Injection port temperature

Detector temperature

Initial and final oven temperatures

Oven temperature programming rate $250^{\circ} \mathrm{C}$

$280^{\circ} \mathrm{C}$

70 or $120^{\circ} \mathrm{C}$ to $250^{\circ} \mathrm{C}$

$20^{\circ} \mathrm{C}$ per minute

\subsection{Determination of Detection Limits of Aromatic Halides}

In order to determine the detection limits of the GC/MS system for various aromatic chlorides, a serious of standard solutions with varying concentrations were prepared. Four types of aromatic chlorides, such as chlorobenzene, 4-chlorobiphenyl, Aroclor 1242, and Aroclor 1254, were dissolved in freshly distilled dry tetrahydrofuran (THF) with concentrations ranging from $0.5 \mathrm{ppm}$ to $10 \mathrm{ppm}$ by weight. The detection limits of the GC/MS system for these chlorinated aromatics are summarized in Table 1.

Table 1. Detection Limits of Chlorinated Aromatics by the GC/MS System

\begin{tabular}{lc}
\hline chlorinated aromatics & $\begin{array}{l}\text { detection limit } \\
\mathrm{ppm}(\mathrm{w} / \mathrm{w})^{\mathrm{a}}\end{array}$ \\
\hline chlorobenzene & $<1$ \\
4-chlorobiphenyl & $<1$ \\
Aroclor 1242 & $<7$ \\
Aroclor 1254 & $<8$ \\
\hline
\end{tabular}

${ }^{8}$ Part per million of chlorinated aromatics by weight in THF.

It is clear from Table 1 that for individual compounds, such as chlorobenzene and 4chlorobiphenyl, a minimum concentration of $1 \mathrm{ppm}$ can be detected by the GC/MS system using total ion current for monitoring. On the other hands, for mixtures, such as Aroclor 1242 and 1254 which contain many congeners, the detection limit of the instrument is reduced to $7-8 \mathrm{ppm}$ due to the presence of lower concentrations of each individual congener in the mixture. 
The sodium naphthalide (NaNAPH) process patented by Goodyear was repeated in order to establish a baseline for comparison with new processes developed in our laboratory. The following experimental procedure is representative.

A 50-mL flask equipped with a nitrogen gas purge system and a magnetic stirring bar coated with glass was charged with $10 \mathrm{~mL}$ of freshly distilled dry THF and $0.23 \mathrm{~g}$ (10 mmol) of sodium metal cut into small pieces. To this sodium metal suspension in THF was added 0.64 $\mathrm{g}(5 \mathrm{mmol})$ of naphthalene with gentle stirring. The formation of a green radical anion solution started in a few minutes and was complete within two hours at room temperature. The concentration of the reagent is ca. $0.5 \mathrm{M}$. Sodium naphthalide prepared by this procedure is relatively stable and can be stored under a nitrogen atmosphere for future use. However, the freshly prepared reagent appears to exhibit higher reactivity for dechlorination of aromatic chlorides.

\subsection{Preparation of Standard Solution of Chlorobenzene in Tetrahydrofuran}

To a $100-\mathrm{mL}$ volumetric flask were charged $51.5 \mathrm{mg}$ of chlorobenzene and $53.0 \mathrm{mg}$ of $n$-decane as an internal standard. The freshly distilled dry THF then was added to the flask until the total volume reached $100 \mathrm{~mL}$. The concentration of the chlorobenzene solution is 515 ppm by volume or $595 \mathrm{ppm}$ by weight. The concentration in terms of the weigh of the chlorinated aromatics relative to the total weight of the sample was utilized throughout this study.

\subsection{Dechlorination of Chlorobenzene by NaNAPH Reagent}

To a $100-\mathrm{mL}$ flask fitted with a nitrogen gas purge system and a magnetic stirring bar coated with glass was added by a syringe $50 \mathrm{~mL}$ of the $595 \mathrm{ppm}$ chlorobenzene solution containing $25.8 \mathrm{mg}(0.23 \mathrm{mmol})$ of chlorobenzene. To this solution was added the freshly prepared NaNAPH solution $(0.5 \mathrm{M})$ until the dark green color of the solution persisted. A total volume of $2.0 \mathrm{~mL}$ of the $\mathrm{NaNAPH}$ reagent was introduced. A small aliquot of the reaction mixture was immediately withdrawn by using a syringe and quenched with water. Analysis by the GC/MS system indicated the dechlorination process was already complete, and chlorobenzene could no longer be detected $(<1 \mathrm{ppm})$. The efficiency of the process is therefore greater than $99.8 \%$. The NaNAPH to chlorobenzene molar ratio is 4 (a minimum ratio of 2 is required), far lower than those reported in the Goodyear patent. The GC/MS ion chromatograms before and after chlorobenzene was treated with the NaNAPH reagent are shown in Figure 16.

Dechlorination of chlorobenzene by using a NaNAPH solution stored under a nitrogen atmosphere at room temperature for 3 days was also studied. It was discovered that a molar ratio of 10 between the NaNAPH reagent and chlorobenzene became necessary. It appeared that decomposition of NaNAPH reagent occurred slowly at room temperature even under the protection of a nitrogen atmosphere. 
Figure 16. GC/MS Ion Chromatograms of Dechlorination of Chlorobenzene by NaNAPH

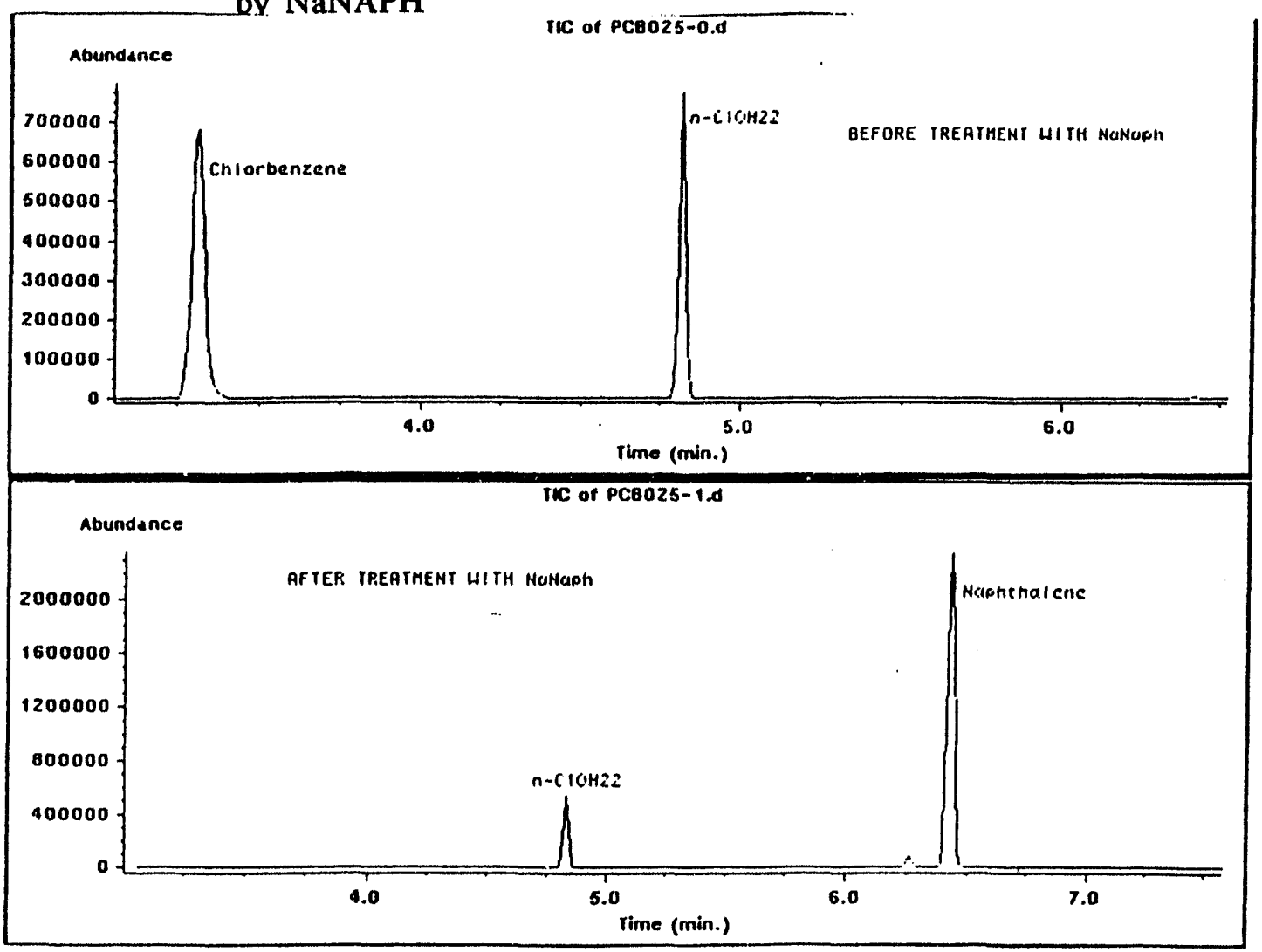

Preparation of Sodium 1-Dimethylaminonaphthalide (NaDMAN)

Preparation of NaDMAN solution in THF is as straightforward as that of NaNAPH. A. $50-\mathrm{mL}$ flask equipped with a nitrogen gas purge system and a magnetic stirring bar coated with glass was charged with $10 \mathrm{~mL}$ of freshly distilled THF and $0.23 \mathrm{~g}(10 \mathrm{mmol})$ of sodium metal cut into small pieces. To this sodium metal suspension in THF was added via a syringe $0.86 \mathrm{~g}$ $(5.0 \mathrm{mmol})$ of 1 -dimethylaminonaphthalene at $-10^{\circ} \mathrm{C}$ with gentle stirring. The formation of a green radical anion solution occurred within minutes and the reaction was complete in about three hours at $-10^{\circ} \mathrm{C}$. The concentration of the reagent was ca. $0.5 \mathrm{M}$. It was observed that this reagent is less stable than NaNAPH at ambient temperature even under the protection of a nitrogen atmosphere. After storing NaDMAN at room temperature for only two to three days, it essentially had lost all its reactivity for the dechlorination reaction.

\subsection{Dechlorination of Chlorobenzene by the NaDMAN Reagent}

The following procedure is representative for dechlorination of chlorinated compounds by the NaDMAN reagent. A $100-\mathrm{mL}$ flask fitted with a nitrogen gas purge system and a magnetic stirring bar coated with glass was charged with $30 \mathrm{~mL}$ of a $635 \mathrm{ppm}$ chlorobenzene solution in THF containing $17 \mathrm{mg}(0.15 \mathrm{mmol})$ of chlorobenzene. To this solution at room temperature was 
added a freshly prepared NaDMAN $(0.5 \mathrm{M})$ until the dark green color of the solution persisted. The total volume of the reagent added was $1.5 \mathrm{~mL}$ (reagent to chlorobenzene molar ratio $=5$ to 1). At this point, the dechlorination process was already complete and chlorobenzene could no longer be detected by the GC/MS system, indicating the concentration of chlorobenzene in the treated solution was less than $1 \mathrm{ppm}$ and the efficiency of the process was better than $99.8 \%$.

\subsection{Dechlorination of 4-Chlorobiphenyl by the NaDMAN Reagent.}

Dechlorination of 4-chlorobiphenyl by the NaDMAN reagent was investigated. Similar result was also obtained with 4-chlorobiphenyl being rapidly dechlorinated from an initial concentration of $585 \mathrm{ppm}$ to less than $1 \mathrm{ppm}$, producing biphenyl as the reaction product. The GC/MS ion chromatograms before and after treatment are shown in Figure 17.

Figure 17. GC/MS Ion Chromatograms of Dechlorination of 4-Chlorobiphenyl

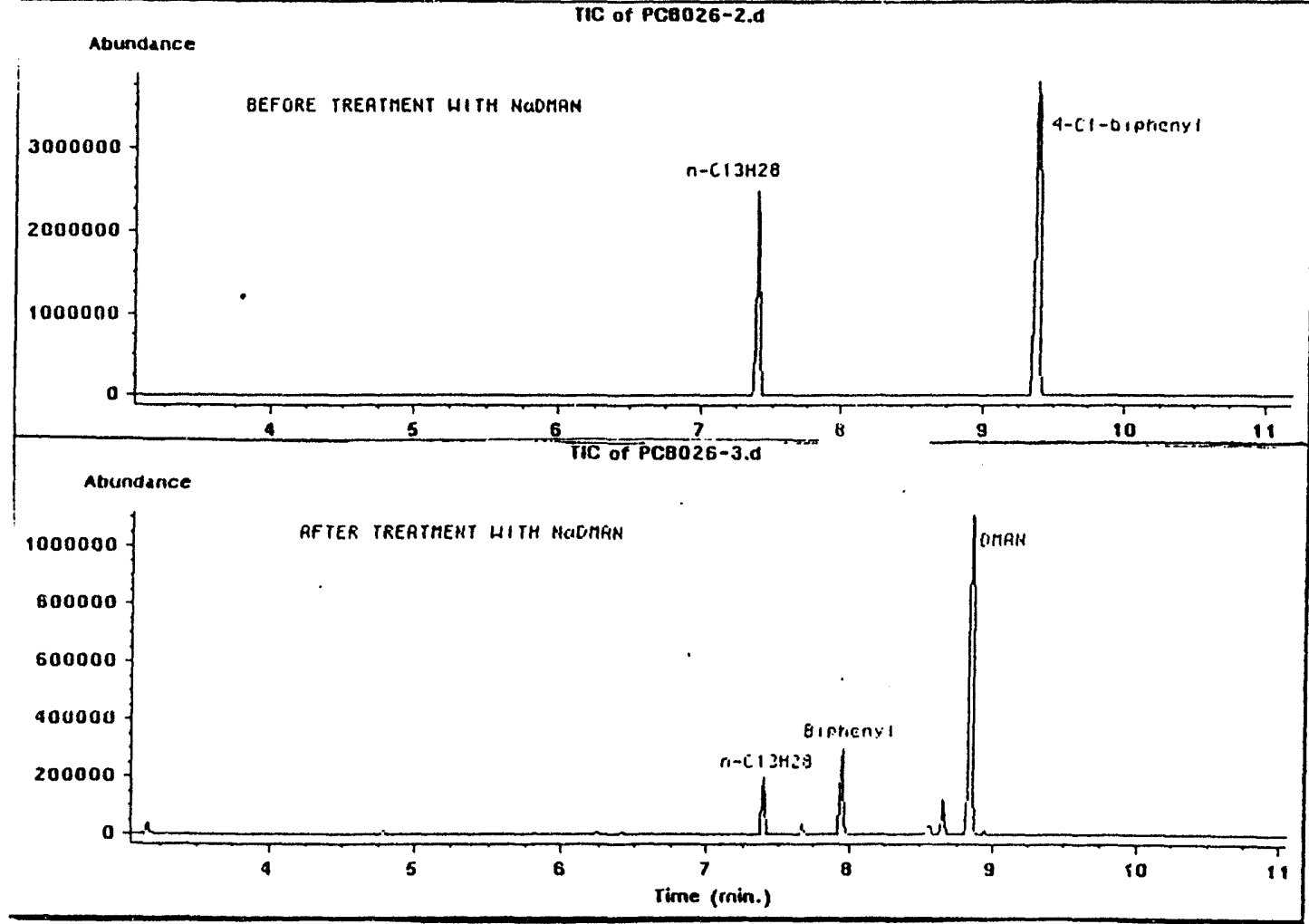

Dechlorination of Aroclor 1242 and Aroclor 1254 by the NaDMAN reagent.

Aroclor 1242 and Aroclor 1254 were purchased from AccuStandard Co. of New Haven, CT. As indicated by the last two digits of the four-digit number, Aroclor 1242 contains approximately $42 \%$ chlorine by weight and Aroclor 1254 has $54 \%$ chlorine by weight. The GC/MS ion chromatograms of these two PCB mixtures are shown in Figure 18 in which the number of chlorine atoms in the congeners are indicated on the top of the peak. The tallest peak was selected to determine the limit of detection. 
Figure 18. GC/MS Ion Chromatograms of Aroclors 1242 and 1254

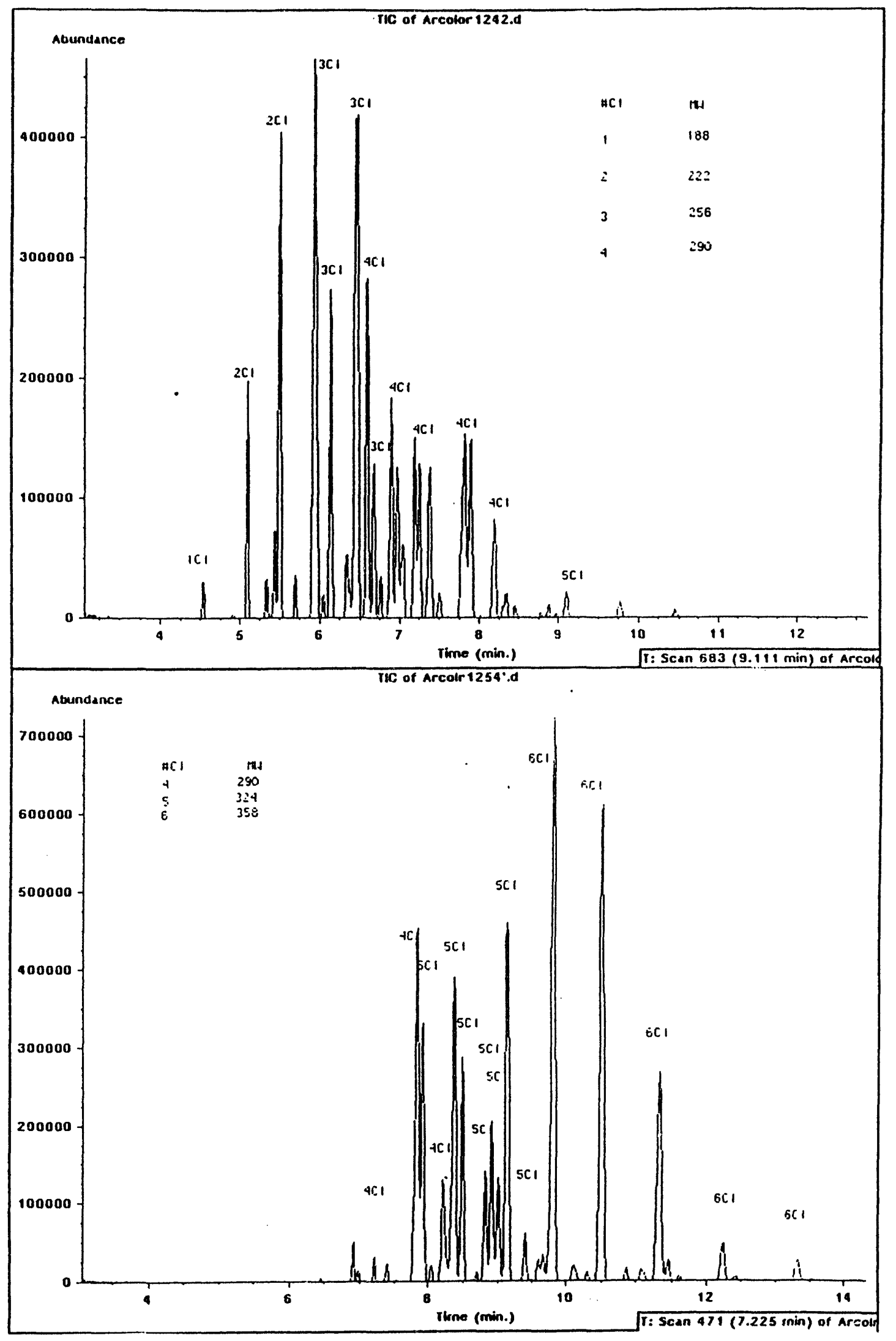


The dechlorination procedure described for dechlorination of chlorobenzene by NaDMAN was also utilized. To $30 \mathrm{~mL}$ of a $614 \mathrm{ppm}$ solution of Aroclor 1242 containing $16 \mathrm{mg}$ of the PCB mixture having $0.19 \mathrm{mmol}$ of chlorine was treated with a $0.5 \mathrm{M} \mathrm{NaDMAN}$ solution until the dark green color persisted. A total volume of $1.5 \mathrm{~mL}$ of NaDMAN was introduced, indicating a molar ratio of ca. 4 to 1 of reagent vs. chlorine content was needed to dechlorinate Aroclor 1242 to less than $8 \mathrm{ppm}$. Similar result was also obtained when $30 \mathrm{~mL}$ of a $583 \mathrm{ppm}$ solution of Aroclor 1254 containing $15.5 \mathrm{mg}$ of the PCB mixture having $0.24 \mathrm{mmol}$ of chlorine was treated with $2 \mathrm{~mL}$ of a $0.5 \mathrm{M}$ solution of NaDMAN (molar ratio between NaDMAN and chlorine content $=$ ca. 4 to 1 ), reducing Aroclor 1254 to less than $7 \mathrm{ppm}$. The GC/MS ion chromatograms of these two experiments are shown in Figures 19 and 20.

Figure 19. GC/MS Ion Chromatograms of Dechlorination of Aroclor 1242 by NaDMAN

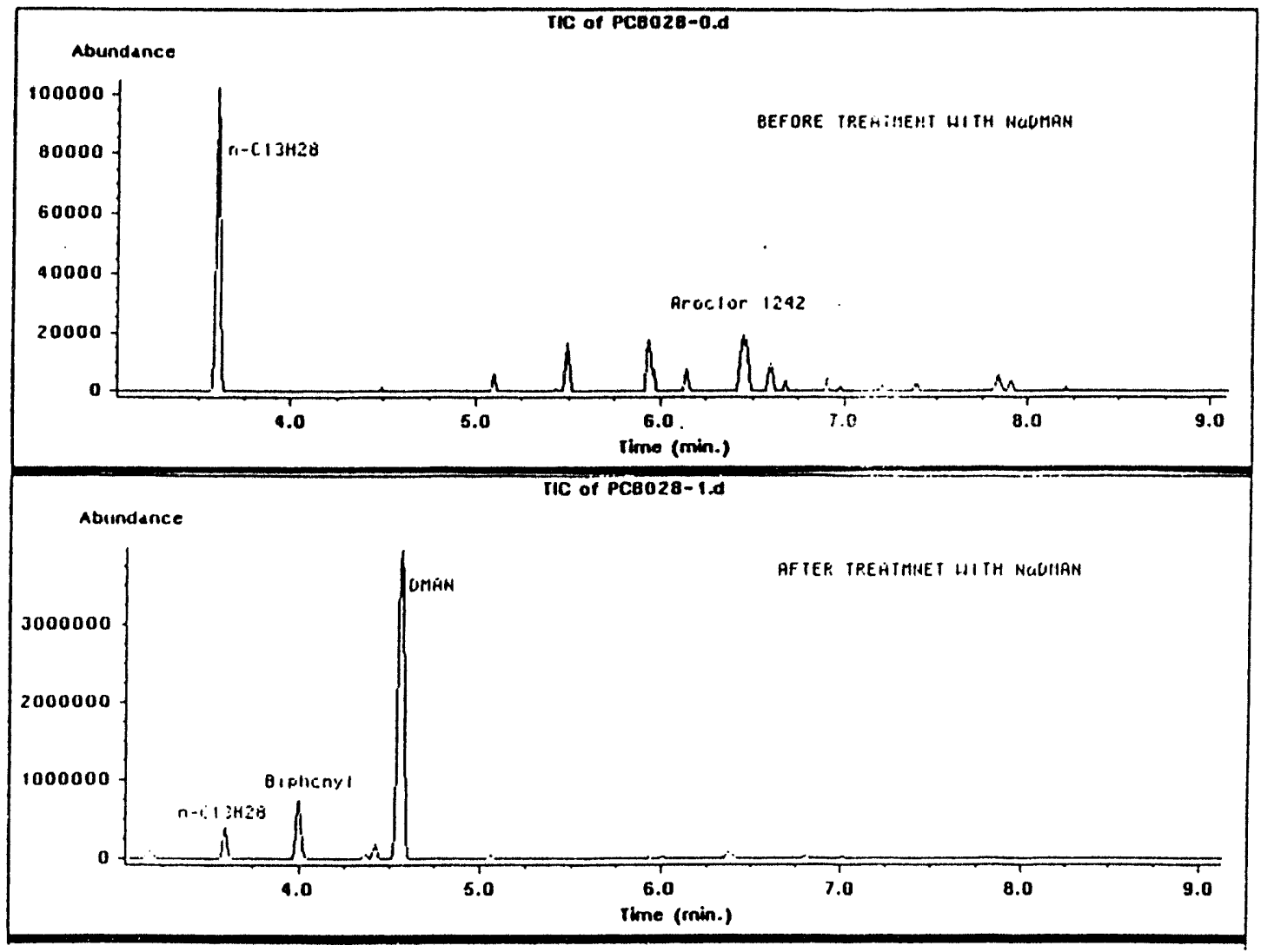


Figure 20. GC/MS Ion Chromatograms of Dechlorination of Aroclor 1254 by NaDMAN

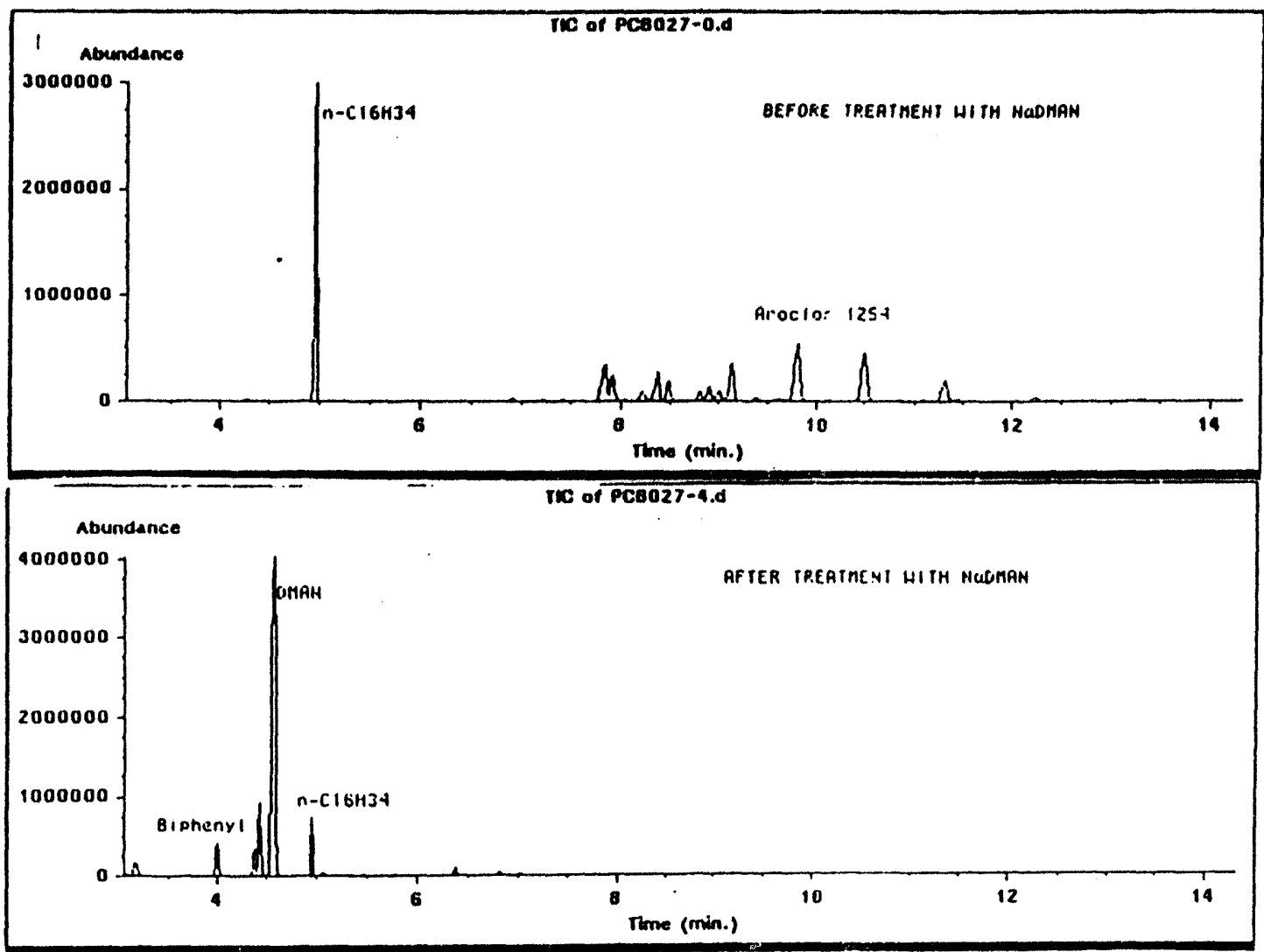

\subsection{Recovery of 1-Dimethylaminonaphthalene}

Recovery of 1-dimethylaminonaphthalene from the reaction mixture was carried out by bubbling a stream of air through the solution until the dark green color disappeared. The mixture was then washed twice with $30 \mathrm{~mL}$ of a $10 \% \mathrm{HCl}$ solution. The combined aqueous layers were treated with a $20 \% \mathrm{NaOH}$ solution until the $\mathrm{pH}$ value became 14 followed by extraction three times with $50 \mathrm{~mL}$ of diethyl ether. The combined ether layers were dried over $\mathrm{MgSO}_{4}$ and the ether solvent was evaporated under vacuum, providing essentially a quantitative recovery of 1dimethylaminonaphthalene with a purity of $90 \%$. 


\subsection{Synthesis of 4-(Dimethylamino)phenyldimesitylborane (22)}

In a 50-mL flask equipped with a nitrogen purge system and a magnetic stirring bar were placed $0.50 \mathrm{~g}(2.5 \mathrm{mmol})$ of 4-bromo- $N, N$-dimethylaniline and $10 \mathrm{~mL}$ of freshly distilled dry THF. The reaction mixture was cooled to $-78^{\circ} \mathrm{C}$ in a dry ice-acetone bath. To this solution was added dropwise via a syringe $1.05 \mathrm{~mL}$ of a $2.5 \mathrm{M}$ solution of $n$-butyllithium in hexanes. After $10 \mathrm{~min}$, the mixture was transferred via cannula to a separate flask containing $0.75 \mathrm{~g}(2.8 \mathrm{mmol})$ of dimesitylboron fluoride dissolved in $5 \mathrm{~mL}$ of dry THF at $-78^{\circ} \mathrm{C}$. The reaction mixture was allowed to warm to room temperature and then was poured into a beaker containing $50 \mathrm{~mL}$ of water and $50 \mathrm{~mL}$ of diethyl ether. The organic layer was separated, and the aqueous layer was extracted with diethyl ether $(3 \times 30 \mathrm{~mL})$. The combined organic layers were washed with water and brine, dried over anhydrous $\mathrm{Na}_{2} \mathrm{SO}_{4}$, and concentrated. The residue was purified by column chromatography (silica gel / hexane) to furnish $0.66 \mathrm{~g}(72 \%)$ of 22 . Further purification by recrystallization from acetonitrile afforded $0.42 \mathrm{~g}$ of a very pure product: $\mathrm{mp} 164-166^{\circ} \mathrm{C}$ (lit. ${ }^{27}$

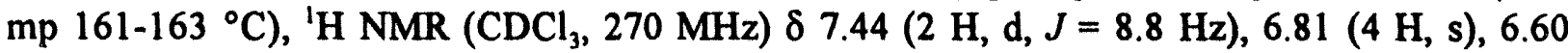
$(2 \mathrm{H}, \mathrm{d}, J=8.8 \mathrm{~Hz}), 3.03(6 \mathrm{H}, \mathrm{s}), 2.30(6 \mathrm{H}, \mathrm{s}), 2.07(12 \mathrm{H}, \mathrm{s})$. The 'H NMR spectrum (270 $\mathrm{MHz}$ ) of 22 is shown in Figure 21.

\subsection{Synthesis of Diphenyl(2,4,6-triisopropylphenyl)borane (28)}

Diphenyl(2,4,6-triisopropylphenyl)borane (28) was successfully synthesized by treating methyl diphenylborinate ${ }^{29}$ with $2,4,6$-triisopropyllithium. ${ }^{31}$ To a $100-\mathrm{mL}$ flask fitted with a magnetic stirring bar and purged with nitrogen were added $20 \mathrm{~mL}$ of dry tetrahydrofuran (THF) as the solvent of the reaction and $1.42 \mathrm{~g}(5.0 \mathrm{mmol})$ of 1-bromo-2,4,6-triisopropylbenzene purchased from Lancaster Synthesis Inc. and the reaction mixture was cooled to $-78{ }^{\circ} \mathrm{C}$. A solution of $n$-butyllithium $(2.5 \mathrm{~mL}, 2.5 \mathrm{M}$ in hexanes, $5.5 \mathrm{mmol})$ was then introduced dropwise over 10 minutes by using a syringe. After 15 minutes of stirring at $-78{ }^{\circ} \mathrm{C}$, the reaction mixture was transferred via cannula to a separate $100-\mathrm{mL}$ flask containing $1.08 \mathrm{~g}(5.5 \mathrm{mmol})$ of methyl diphenylborinate in $15 \mathrm{~mL}$ of dry THF kept at $-78^{\circ} \mathrm{C}$. The reaction mixture was then allowed to warm to room temperature and stirred overnight. After $50 \mathrm{~mL}$ each of pentane and water were introduced, the organic layer was separated and the aqueous layer was extracted with pentane (2 x $25 \mathrm{~mL}$ ). The combined organic layers were concentrated, and the residue was purified by column chromatography (silica gel / hexanes) to afford $0.708 \mathrm{~g}$ (39\% yield) of diphenyl $(2,4,6-$ triisopropylphenyl)borane (28) as a white solid: IR $2960,2866,1591,1434,1237,1184,876$, 747, $700 \mathrm{~cm}^{-1}$; ' $\mathrm{H} \mathrm{NMR}\left(\mathrm{CDCl}_{3}, 270 \mathrm{MHz}\right) \delta 7.67(4 \mathrm{H}, \mathrm{dd}, J=8.0$ and $1.6 \mathrm{~Hz}), 7.52-7.40(6$ $\mathrm{H}, \mathrm{m}), 7.00(2 \mathrm{H}, \mathrm{s}), 2.94(1 \mathrm{H}$, septet, $J=7.0 \mathrm{~Hz}), 2.37(1 \mathrm{H}$, septet, $J=6.8 \mathrm{~Hz}), 1.32(6 \mathrm{H}$, d, $J=7.0 \mathrm{~Hz}), 0.96(12 \mathrm{H}, \mathrm{d}, J=6.8 \mathrm{~Hz})$; MS m/e $368\left(\mathrm{M}^{+}\right), 353,325,290,247,233,191,169$. The ' $\mathrm{H}$ NMR spectrum (270 MHz) and the GC/MS of diphenyl(2,4,6-triisopropylphenyl)borane (28) are shown in Figures 22 and 23, respectively. 
Figure 21. The 'H NMR Spectrum $\left(\mathrm{CDCl}_{3}, 270 \mathrm{MHz}\right)$ of 22

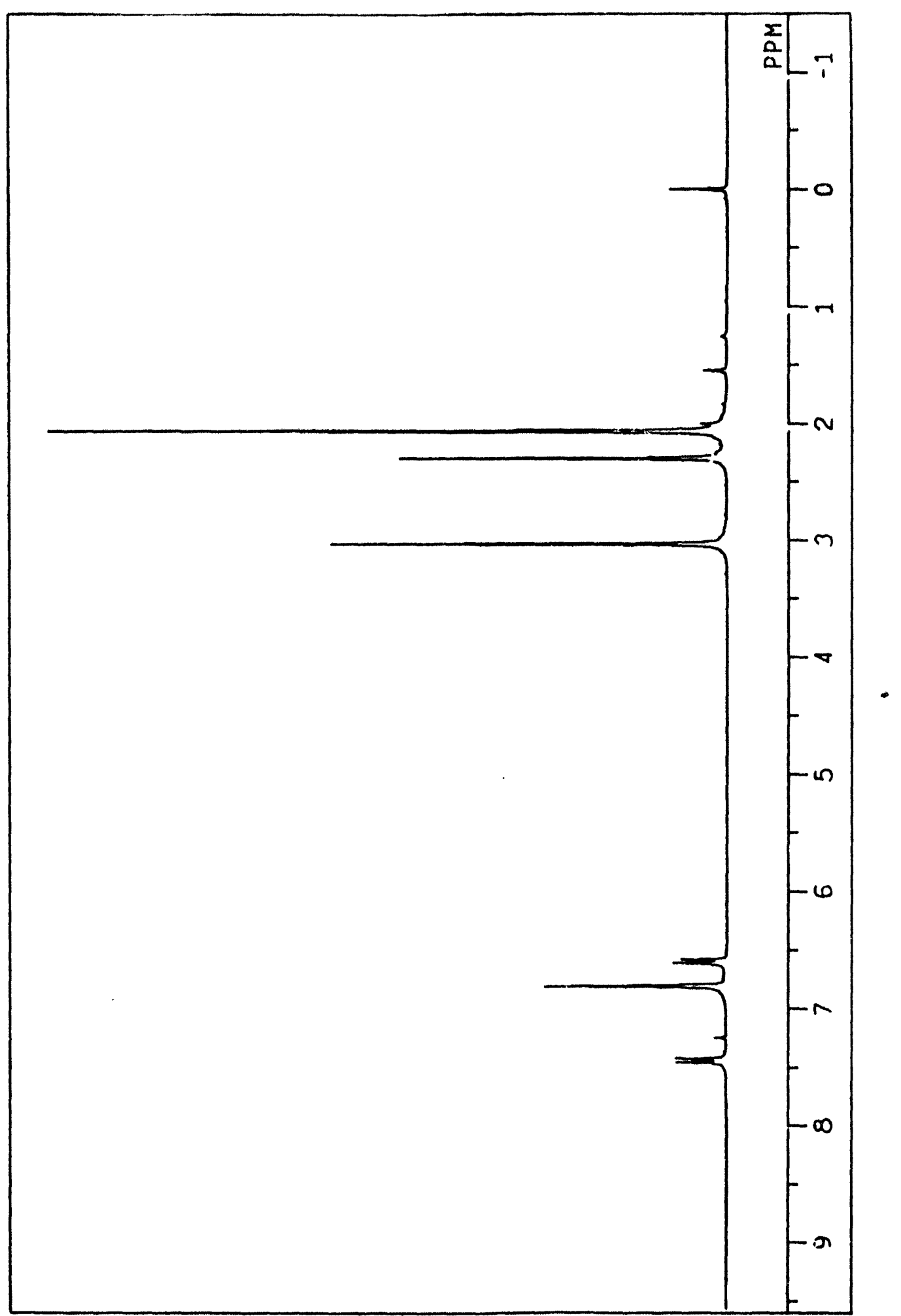


Figure 22. The 'H NMR Spectrum $\left(\mathrm{CDCl}_{3}, 270 \mathrm{MHz}\right)$ of

Diphenyl(2,4,6-triisopropylphenyl)borane (28)

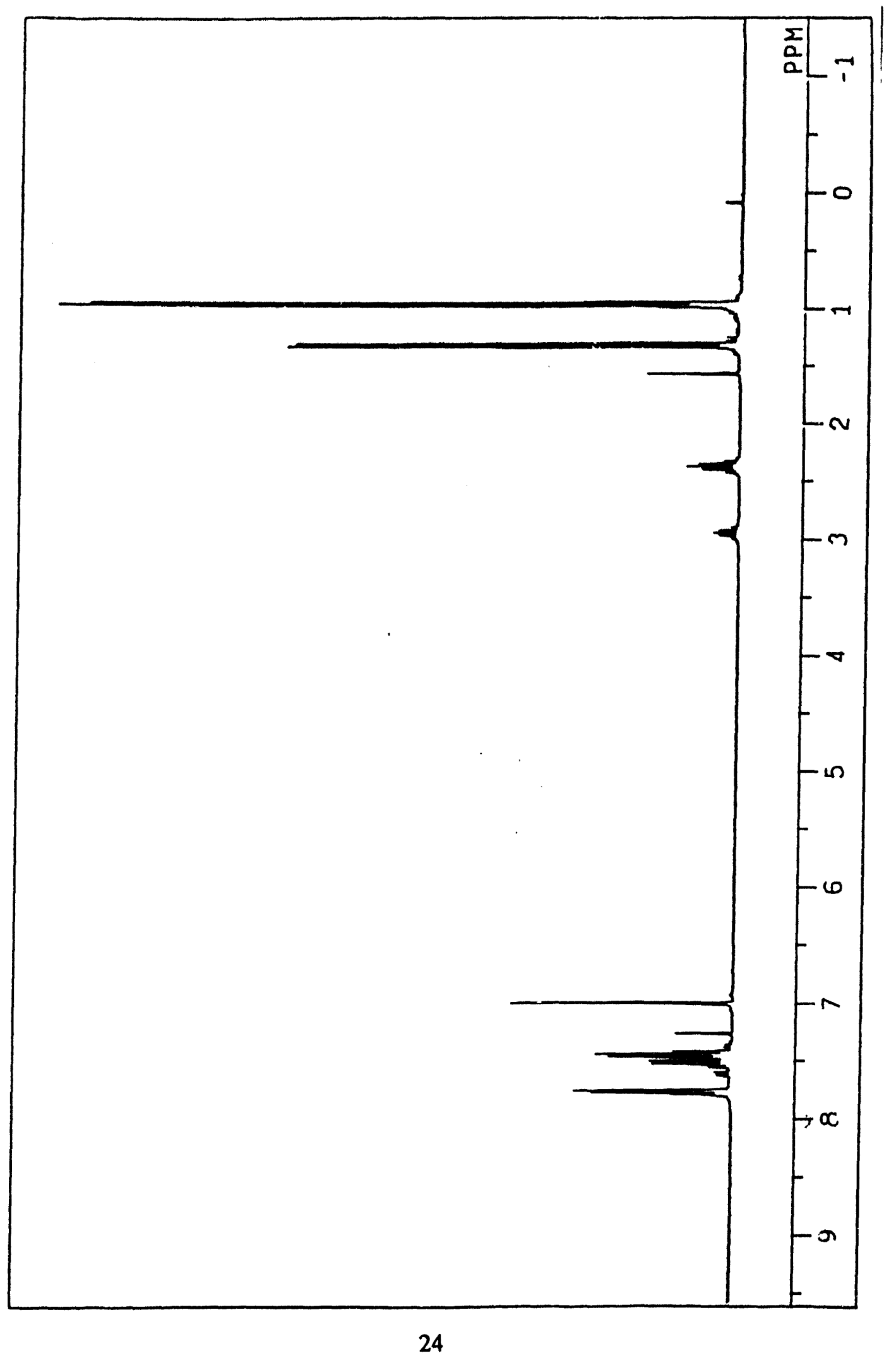




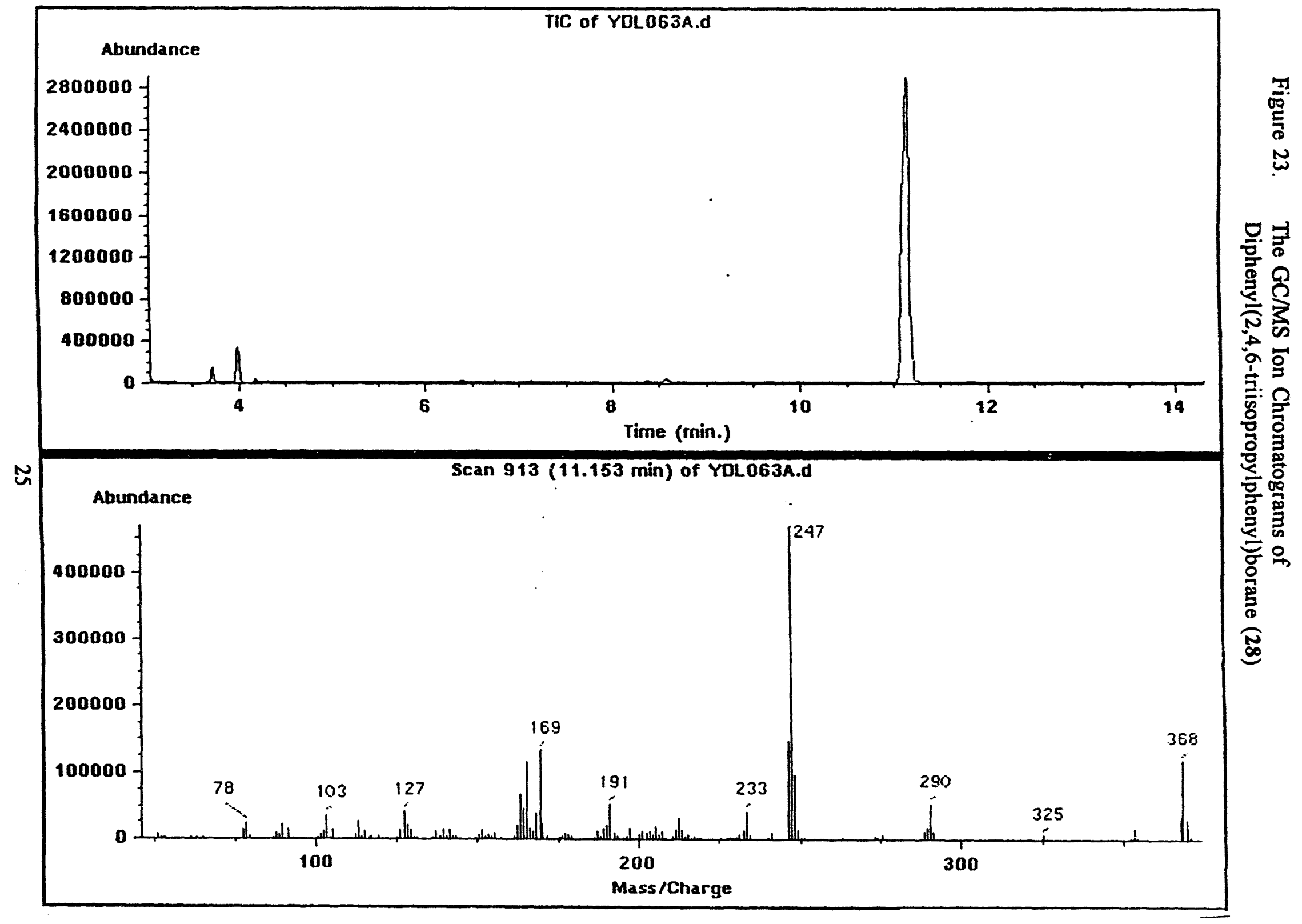




\section{CONCLUSIONS}

In this report, four general methods for chemical destruction of PCBs have been reviewed. These technologies have achieved limited success in some commercial-scale operations. The laboratory scale studies suggest that these remediation processes have good potential for effective dechlorination of PCBs. In our laboratory at West Virginia University, we have successfully utilized 1-dimethylaminonaphthalene as an electron carrier for sodium metal for dechlorination of aromatic halides. The easy recovery of 1-dimethylaminonaphthalene from the reaction mixture for recycle offers a significant advantage over other electron carriers, such as naphthalene. Currently, efforts are being made to synthesize sterically hindered and chemically more stable triarylboranes as potential electron carriers for sodium metal. Attachment of the electron carrier to a polymer backbone to allow easy separation after the dechlorination reaction is also being investigated. Continued research in the area of chemical remediation is still needed to adapt these chemical processes to an extremely complex problem of $\mathrm{PCB}$ pollution in the environment. The PCB pollutant in the environment may exist in the soil matrices, in aqueous solution and in transformer oil with varying concentrations, and may coexist with other pollutants. It is unlikely that one single chemical remedial procedure will be able to address all these problems. A variety of procedures need to be developed for effective removal of PCBs from the environment. 


\section{BIBLIOGRAPHY}

(1) Bailey, R.A.; Clarke, H.M.; Ferris, J.P.; Krause, S.; Strong, R.L., Chemistry and the Environment, Academic Press: New York, 1978; p 158.

(2) See reference $1 \mathrm{pp} \mathrm{172-174.}$

(3) Hanson, D.J., Chemical and Engineering News 1993, 71, 7.

(4) (a) Sworzyn, E.M.; Ackerman, D.G., U.S. Environmental Protection Agency, Report No. EPA-600/2-82-069. (April 1982)

(b) Fradkin, L.; Barisas, S., Argonne National Laboratory, Argonne, IL, Report No. ANL/EES-TM-168 (NTIS No. DE 82013715) (1982).

(5) Erickson, M.D., Analytical Chemistry of PCBs, Lewis Publishers, Inc.: Michigan, 1991.

(6) Parker, D.K.; Steichen, R.J., The Goodyear Tire and Rubber Company, Akron, Ohio, U.S. Patent No. 4,284,516, August 18, 1981.

(7) House, H.O., Modern Synthetic Reactions, 2nd Ed., W.A. Benjamin, Inc.: Menlo park, CA 1972; p 217.

(8) Parker, D.K.; Sabo, T.A., The Goodyear Tire and Rubber Company, Akron, Ohio, U.S. Patent No. 4,447,667, May 8, 1984.

(9) Chen, K., IEEE Transactions on Power Apparatus and Systems 1983, PAS-102, 3893.

(10) Hawari, J.A.; Samson, R., National Research Council of Canada, Ottawa, Canada, U.S. Patent No. 4,950,833, August 21, 1990.

(11) Griller, D.; Hawari, J.A., National Research Council of Canada, Ottawa, Canada, U.S. Patent No. 4,973,783, November 27, 1990.

(12) See reference 7 pp 537-540.

(13) Gruber, W. EI Digest June 1991, 6.

(14) Rittenhouse, R.C. Power Engineering July 1991, 10.

(15) Berg, O.W.; Drosody, P.L.; Pees, G.A.V. Bull. Environm. Contam. Toxicol. 19727 , 338.

(16) Lapierre, R.B., U.S. Environmental Protection Agency Report No. 60013-77-018 (January 1977). 
(17) Dennis, W.H., Jr.; Chang, Y.H.; Cooper, W.J. Bull. Environm. Contam. Toxicol. $1979,22,750$.

(18) Colon, I., Union Carbide Corporation, Danbury, Conn., U.S. Patent No. 4,400,566, August 23, 1983.

(19) (a) Epling, G.A.; McVicar, W.M.; Kumar, A. Chemosphere 1988, 17, 1355.

(b) Tsujimoto, K.; Tasaka, S.; Ohashi, M. J. Chem. Soc., Chem. Commun. 1975, 758.

(c) Epling, G.A.; Florio, R. Tetrahedron Lett. 1986, 27, 675.

(20) Ohashi, M.; Tsujimoto, K; Seki, K. J. Chem. Soc., Chem. Commun. 1973, 384

(21) (a) Mochizuki, S. Chemical Engineering Science 1977, 32, 1205.

(b) Nishiwaki, T.; Usui, M.; Knda, K.; Hida, M. Bull. Chem. Soc., Jpn 1979, 52,821 .

(22) Chaudhary, S.K.; Mitchell, R.H.; West, P.R. Chemosphere 1984, 13, 1113.

(23) Hawari, J.; Demeter, A.; Greer, C.; Samson, R. Chemosphere 1991, 22, 1161-1174.

(24) Bank, S.; Platz, M. Tetrahedron Lett. 1973, 2097-2100.

(25) (a) Chu, T.L.; Weismann, T.J. J. Am. Chem. Soc. 1956, 78, 23.

(b) Darling, S.D.; Devgan, O.N.; Cosgrove, R.E. J. Am. Chem. Soc. 1970, 92, 696.

(26) Leffler, J.E.; Watts, G.B.; Tanigaki, T.; Dolan, E.; Miller, D.S. J. Am. Chem. Soc. 1970, 92,6825 .

(27) Doty, J.C.; Babb, B.; Grisdale, P.J.; Glogowski, M.; Williams, J.L.R. J. Organomet. Chem. 1972, 38, 229.

(28) Brown, H.C.; Dodson, V.H. J. Am. Chem. Soc. 1957, 79, 2302.

(29) Jacob, P., III J. Organomet. Chem. 1978, 156, 101.

(30) Pachaly, B.; West, R. J. Am. Chem. Soc. 1985, 107, 2987.

(31) Millevolte, A.J.; Powell, D.R.; Johnson, S.G.; West, R. Organometallics 1992, 11, 1091. 
Development of Organic Sensors: Monolayer and Multilayer Self-Assembled Films for Chemical Sensors

(Project MC-7)

\author{
Quarterly Report for Period \\ for Period October 1 through December 31, 1993
}

Work Performed Under Contract

No.: DE-FC21-92MC29467

\author{
For \\ U.S. Department of Energy \\ Office of Fossil Energy \\ Morgantown Energy Technology Center \\ Morgantown, West Virginia
}

\author{
By \\ Harry O. Finklea \\ Department of Chemistry \\ West Virginia University \\ Morgantown, West Virginia
}

February 1994 


\section{TABLE OF CONTENTS}

$1.0 \quad$ EXECUTIVE SUMMARY $\ldots \ldots \ldots \ldots \ldots \ldots \ldots \ldots \ldots \ldots$

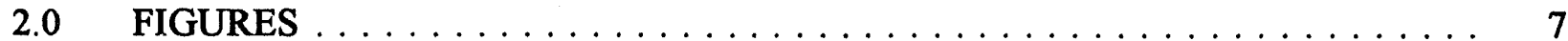




\subsection{EXECUTIVE SUMMARY}

\section{Synopsis:}

Work this quarter focused on depositing multilayer polyion films on SAW crystals, improving the design of the environmental chamber around the mass microbalance, and calibrating the flow of benzene vapor from the vapor generator. Deposition of polyion layers was tested on model substrates (hydrophobic $\mathrm{SiO}_{x}$ and hydrophilic $\mathrm{Al}_{2} \mathrm{O}_{3}$ ). A preliminary experiment was performed on the response of a 7-layer film to $100-300 \mathrm{ppm}$ benzene vapor.

\section{Results:}

As noted in the preceding quarterly report, the SAW crystals, purchased from Femtometrics, are easily damaged when placed in contact with solutions. The problem appears to be chemical attack of the aluminum pads and electrodes which lie on the surface of the quartz crystal. Both acidic and basic conditions in aqueous solutions cause both dissolution and delamination of the Al electrodes. We tested several strategies to improve the reliability of film deposition on the SAW crystals.

First, all deposition solutions were adjusted to $\mathrm{pH} 7$ with a buffer; usually solid $\mathrm{NaHCO}_{3}$ was added until the $\mathrm{pH}$ was $7 \pm 1$ according to $\mathrm{pH}$ paper. At $\mathrm{pH} 7$ a sticking layer based on aliphatic amine groups (see below) is charged due to protonation of the amines. The necessity of buffering the solutions introduced an uncertainty about the type of counter ions in the polylayers. Poly(styrene sulfonate, sodium salt) (abbreviated $\mathrm{PSSNa}^{+}$) solutions typically contained $0.02 \mathrm{M}$ concentration of the monomer unit, and $0.01 \mathrm{M} \mathrm{NaHCO}_{3}$; the dominant cation in the deposited polyion layer was $\mathrm{Na}^{+}$. However, solutions of poly(vinylmethylpyridinium methylsulfonate) (PVPMe ${ }^{+} \mathrm{CH}_{3} \mathrm{SO}_{3}{ }^{-}$), as prepared in our lab., contained $0.02 \mathrm{M}$ of the monomer unit and nearly $0.3 \mathrm{M} \mathrm{NaHCO}_{3}$ because of the excess acid present in the polymer after synthesis. Consequently the dominant counter ion in the $\mathrm{PVPMe}^{+}$polyion layer was probably $\mathrm{HCO}_{3}$. Future work will require a better method for controlling the counter ion composition as well as the $\mathrm{pH}$ of the solutions.

Second, an attempt was made to deposit polyion multilayers on a hydrophobic silica surface. Addition of a hydrophobic silane layer to the surface of the SAW crystal may reduce the penetration of water to the aluminum pads and therefore improve their chemical resistance. A reprint from Rubner at MIT [1] indicate that polyion layers can be deposited on hydrophobic as well as hydrophilic substrates provided that the first polyion layer is a polycation. To make the silicon wafer hydrophobic, the wafer was exposed to trimethylchlorosilane $\left(\mathrm{Me}_{3} \mathrm{SiCl}\right)$ vapors for two days in a sealed dessicator. The hydrophilic oxide surface of the clean wafer became intensely hydrophobic. Ellipsometry indicated the presence of a $5 \AA$ film, consistent with a monolayer of $\mathrm{Me}_{3} \mathrm{Si}$ on the $\mathrm{SiO}_{x}$ surface. Polyion deposition of $\mathrm{PVPMe}^{+}$(15 minutes in the polyion solution, rinse with water and dry) followed by PSS (same protocol) yielded an average thickness increment of $9 \pm 4 \AA$ until the 9th layer. At that point no further polyion layers could be deposited. 
In a related experiment, a silicon wafer was modified with a octadecyltrichlorosilane (OTS) monolayer. This modification also produced an intensely hydrophobic surface with $24 \AA$ thickness. The modified wafer was exposed to an air plasma using the Harrick Plasma generator. The air plasma was intended to partially oxidize the hydrocarbon surface of the monolayer and provide acidic groups which would promote binding of polyion layers. Indeed, the post-plasma thickness of the monolayer was $4 \AA$, and the surface was nearly hydrophilic. However, exposure to a polycation solution resulted in no perceptible binding of the polycation to the substrate.

Third, the silane with pendant amine groups $\left((\mathrm{MeO})_{3} \mathrm{SiCH}_{2} \mathrm{CH}_{2} \mathrm{NHCH}_{2} \mathrm{CH}_{2} \mathrm{NH}_{2}\right.$, abbreviated as en-silane) was deposited on the SAW device in the vapor phase rather than from solution. Two procedures were tested. In the first procedure, the liquid silane was placed in a bubbler and dry air was passed through the liquid, generating air saturated with the silane. This air stream was diluted 1:1 with dry air to avoid problems with condensation of the silane downstream. The vapor pressure of this silane at room temperature is low, on the order of 0.5 torr. The air + silane was passed over the SAW crystal while the crystal was operating in the mass microbalance. The frequency change $\Delta f$ was monitored continuously to follow the adsorption of the silane onto the crystal. Figure 1 shows the first 4 hours of operation in which the silane flow was turned on and off at half-hour intervals. After a 50 minute period of operation to establish a baseline signal, $\Delta f$ rose rapidly on exposure to the silane. Some of the silane was weakly adsorbed as indicated by the drop in signal when only dry air flowed across the SAW crystal. The exposure-and-purge cycles were repeated in order to assist the removal of reaction products (methanol) and to increase the coverage of the silane layer. We estimate that a close-packed monolayer on the crystal (about $6 \times 10^{-10} \mathrm{~mol} / \mathrm{cm}^{2}$ ) should cause a $\Delta \mathrm{f}$ increase of $20-30 \mathrm{ppm}$ based on a published sensitivity for a $200 \mathrm{MHz}$ SAW device of $4 \mathrm{ng} / \mathrm{cm}^{2} / \mathrm{ppm} \mathrm{[2]}$. After 3 exposure-and-purge cycles it was evident that only a fraction of a monolayer of the silane had bonded to the SAW crystal. Repetition of the exposure-and-purge cycles over 6 more hours yielded a net $\Delta \mathrm{f}$ of $12 \mathrm{ppm}$. This approach, although useful because of the ease of following the silanization reaction, was abandoned because of the length of time that the vapor generator and mass microbalance were tied up.

In the second procedure, the crystal was placed in a sealed dessicator which also contained an open beaker of the liquid silane. After 5 to 6 days, the crystal was removed and purged with dry air. Frequencies were measured before and after the silanization procedure to estimate the coverage. $\Delta$ f of $110 \mathrm{ppm}$ and $30 \mathrm{ppm}$ were obtained on two different SAW crystals, indicating coverages equal to or greater than a monolayer. The main point, however, is that the electrodes on the SAW crystal were not noticeably damaged by the vapor phase silanization.

Work continued on the deposition of the polyion layers both on the SAW crystals and on model substrates. One question is whether the silanization and multilayer deposition occurs on any exposed aluminum electrodes as well as on the $\mathrm{SiO}_{\mathbf{x}}$ protecting layer. We obtained some silicon wafers with sputtered aluminum layers from Dr. Watiq Abdul-Razzaq in the WVU Physics Dept. The following table shows the successful deposition of both the ensilane (deposited from dilute toluene solution) and the polyion layers on the aluminum surface as measured by ellipsometry: 
Táble 1

Ellipsometric thicknesses of silane + polyion layers on an Al surface.

$\begin{array}{lrr}\text { Layer } & \underline{\mathrm{d}(\AA)} & \underline{\mathrm{d}(\AA)} \\ \text { en-silane } & 9 & 9 \\ \text { PSS\#1 } & 18 & 9 \\ \text { PVPMe\#1 } & 31 & 13 \\ \text { PSS\#2 } & 52 & 21 \\ \text { PVPMe\#2 } & 70 & 18 \\ \text { PSS\#3 } & 93 & 23 \\ \text { PVPMe\#3 } & 111 & 18\end{array}$

Deposition of polyion layers on SAW crystals was less successful; in three attempts we succeeded in depositing 6 layers ( 3 polyanion, 3 polycation) only once. In the other two instances, the crystal no longer oscillated after the 4th layer, and in one case the Al electrodes were visibly damaged. We note that the surviving SAW crystal had the extra thick silane layer on the surface; thicker sticking layers may be the key to polyion deposition without damage to the SAW crystal electrodes.

In each of the three experiments with SAW crystals, the crystal oscillation frequency was checked after exposing the crystal to a dry air purge for at least 30 minutes. Table 2 summarizes the changes in $\Delta f$ observed after the deposition of each layer.

Table 2

Changes in beat frequency after deposition of silane and polyion layers.
Crystal \#1
Crystal \#2
Crystal \#3

\begin{tabular}{|c|c|c|c|c|c|}
\hline Layer & $\underline{\Delta f(p p m)}$ & $\underline{\Delta(\Delta f)}$ & $\underline{\Delta f(p p m)}$ & $\underline{\Delta(\Delta f)}$ & $\Delta \mathrm{f}(\mathrm{ppm})$ \\
\hline en-silane & 10 & 10 & 113 & 113 & 29 \\
\hline PSS\#1 & 72 & 62 & 169 & 56 & 62 \\
\hline cation* & 82 & 10 & 248 & 79 & 107 \\
\hline PSS\#2 & 128 & 46 & 302 & 54 & 150 \\
\hline cation* & 147 & 19 & 339 & 37 & 134 \\
\hline PSS\#3 & $!$ & & 385 & 46 & $!$ \\
\hline cation* & & & 442 & 57 & \\
\hline
\end{tabular}

*The polycation is either polyallylamine (which is protonated at $\mathrm{pH}$ 7) for Crystal \#1 or PVPMe for Crystals \#2 and \#3. At! the crystals stopped oscillating.

A graph of $\Delta \mathrm{f}$ for each deposited layer on Crystals \#1 and \#2 is shown in Figure 2. The PSS layers consistently produce changes in $\Delta f$ of $50 \pm 10 \mathrm{ppm}$, corresponding to mass increases of $2 \times 10^{-7} \mathrm{~g} / \mathrm{cm}^{2}\left(1 \times 10^{-9} \mathrm{~mol}\right.$ of monomer unit per $\left.\mathrm{cm}^{2}\right)$, assuming a mass sensitivity of $4 \mathrm{ng} / \mathrm{cm}^{2} / \mathrm{ppm}$ 
and assuming that all of the change in $\Delta \mathrm{f}$ is due to mass changes as opposed to elasticity changes [3]. The mass change due to the polycation is somewhat more variable. Poly-(allylamine) layers appear to be disproportionally smaller in mass $\left(6 \times 10^{-8} \mathrm{~g} / \mathrm{cm}^{2} ; 5 \times 10^{-10} \mathrm{~mol} / \mathrm{cm}^{2}\right)$ than the PVPMe layers, which have essentially the same coverage as the PSS layers. These mass changes are consistent with approximately a monolayer of polyion being adsorbed in each deposition step.

Crystal \#2 and an uncoated crystal were tested for their response to low concentrations of benzene in the air at $25^{\circ} \mathrm{C}$. Figure 3 shows the response of the uncoated crystal to ca. 100 ppm benzene in dry air. While the initial flux of benzene appeared to cause a positive change in $\Delta f$, removal of benzene from the air did not reverse the trend. Examination of repeated exposures to $100 \mathrm{ppm}$ benzene and clean dry air lead to the conclusion that the $\Delta \mathrm{f}$ changes did not correlate with the presence of benzene at this concentration. The same conclusion was obtained when the air stream had $50 \%$ relative humidity. Likewise, Crystal \#2 with the polyion multilayers shows no significant response to either $100 \mathrm{ppm}$ or $330 \mathrm{ppm}$ benzene in dry air (Figure 4 ) or in air with $50 \%$ relative humidity. The response of the uncoated crystal and Crystal \#2 to the change in relative humidity was $5 \mathrm{ppm}$ and $110 \mathrm{ppm}$ respectively; again the SAW device and the polyion films are very sensitive to the relative humidity, which may be an interference to the sensor. Future work will focus on the incorporation of counter ions to promote the adsorption of the targeted organics.

An environmental chamber was constructed to house the mass microbalance. The chamber is an insulated box containing a circulation fan and a heat exchanger. Water from the constant temperature bath unit circulates through the heat exchanger and through a coil in direct contact with the small metal chamber surrounding the sample ard reference SAW crystals. This improvement in design affords much better temperature control of both the SAW crystals and the air stream passing over the SAW crystals.

A procedure for calibrating the vapor concentration of the organic was developed. The Kintek vapor generator has permeation tubes that are placed in ovens. The permeation tubes contain the liquid organic (e.g. benzene) and a semipermeable membrane. Diffusion of the organic vapor across the membrane and into the airstream is constant at a given temperature, but increases rapidly with temperature. Kintek provides a table of calibrated leak rates at given temperatures; the concentrations of benzene above are based on the Kintek numbers. We checked the concentrations by measuring the weight loss of the permeation tube after prolonged vapor generation at a constant oven temperature. At an oven temperature of $100^{\circ} \mathrm{C}$ and an air flow of $0.25 \mathrm{~L} / \mathrm{min}$, the predicted benzene concentration was $4100 \mathrm{ppm}$ and the observed concentration was $3100 \mathrm{ppm}$. At an oven temperature of $30^{\circ} \mathrm{C}$ and the same air flow, the predicted and measured concentrations were identically $32 \mathrm{ppm}$. These measurements confirm the ability of the vapo: generator to provide organic vapor concentrations in the $1-1000 \mathrm{ppm}$ range. 


\section{References:}

1. Cheurig, J. H.; Fou, A. F.; Rubner, M. F. Thin Solid Films, accepted for publication.

2. Grate, J. W.; Klusty, M. Anal. Chem. 1991, 63, 1719-27.

3. Grate, J. W.; Klusty, M.; McGill, R. A.; Abraham, M. H.; Whiting, G.; AndonianHaftvan, J. Anal. Chem. 1992, 64, 610-24. 


\subsection{FIGURES

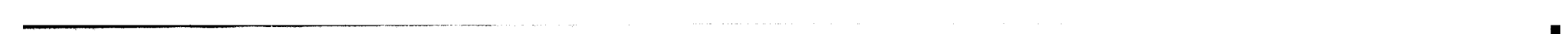




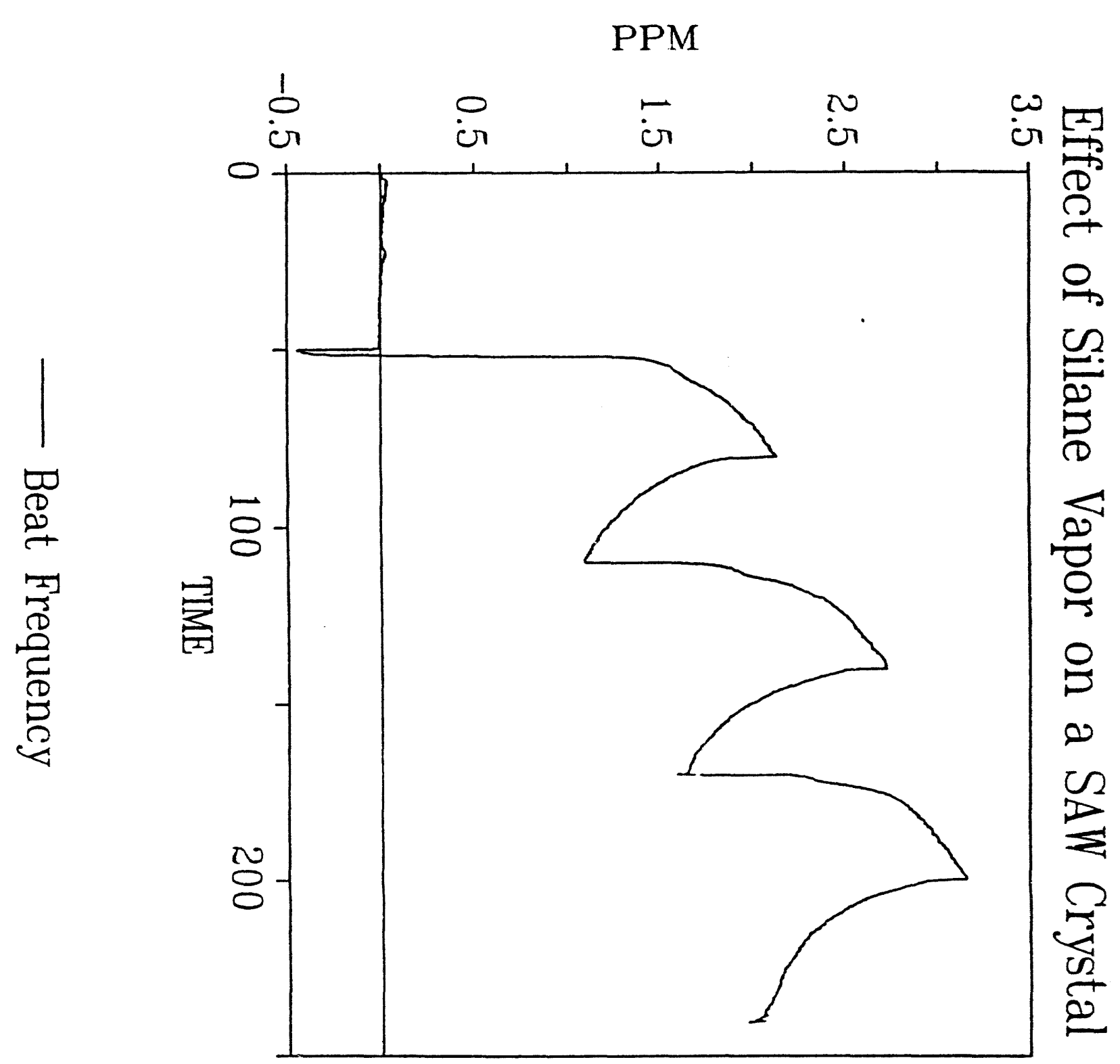


Figure 2

Correlation of Two SAW Crystals Different Silane Layering Process

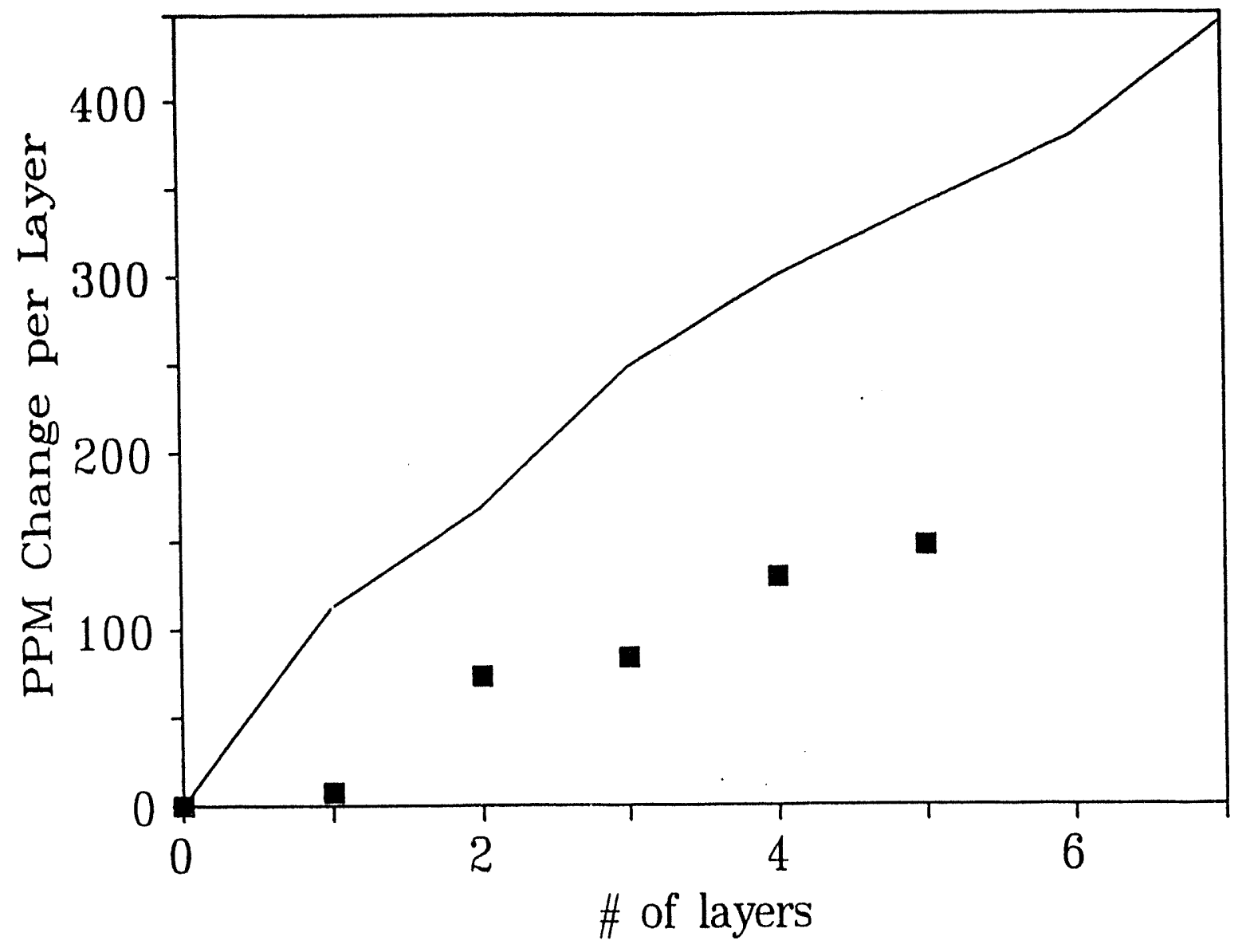

— Vapor Sil/PSS/PVP+ • BubblingSil/PSS/PAA 
Figure 3

Enlargement of First 400 min. p1207-01 Effects of Benzene on Blank SAW Crystal

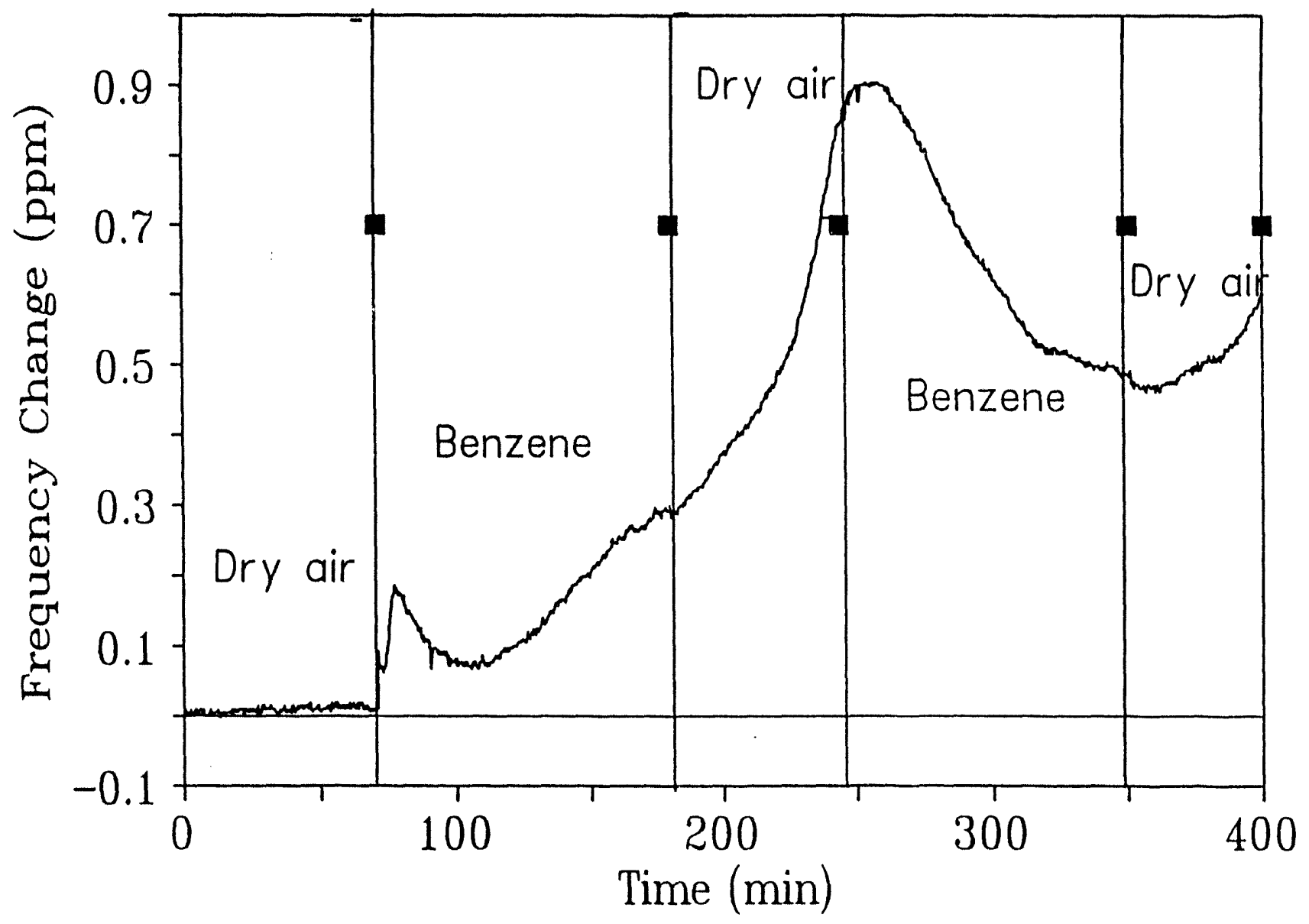

— Beat Freq

- Changing Points 
Figure 4

Enlargement of First 180 min.

Dry Air/Benzene Effects on Coated SAW

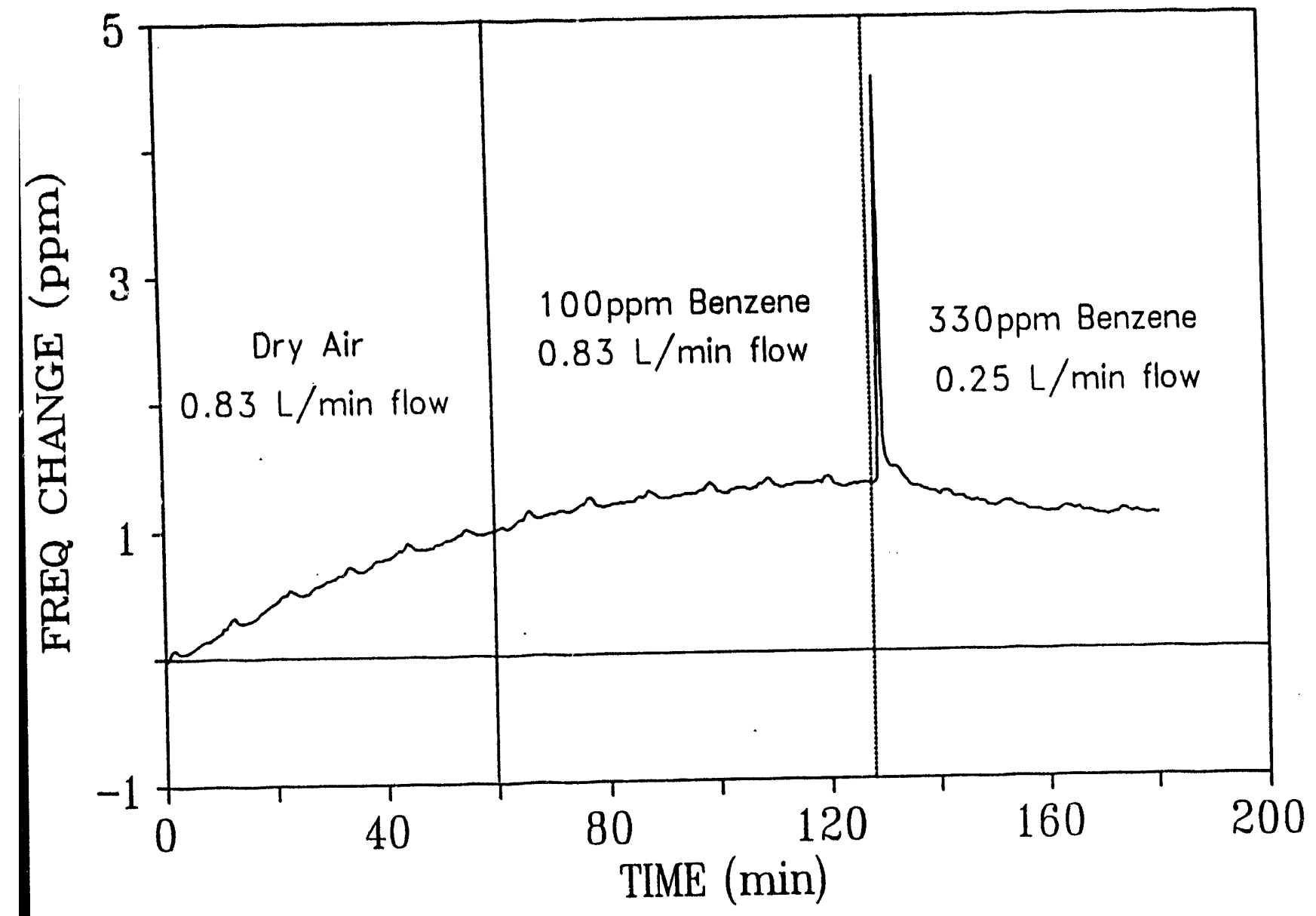

- Beat Freq 


\title{
Winfield Lock and Dam Remediation (Project MC-9)
}

\section{Quarterly Report for Period}

for Period October 1 through December 31, 1993

Work Performed Under Contract

No.: DE-FC21-92MC29467

\section{For}

U.S. Department of Energy

Office of Fossil Energy

Morgantown Energy Technology Center

Morgantown, West Virginia

\author{
By \\ Raymond J. Lovett \\ National Research Center for Coal and Energy \\ Environmental Technology Division \\ West Virginia University \\ Morgantown, West Virginia
}

February 1994 


\section{Table of Contents}

EXECUTIVE SUMMARY $\ldots \ldots \ldots \ldots \ldots \ldots \ldots \ldots \ldots \ldots \ldots \ldots \ldots \ldots \ldots \ldots$

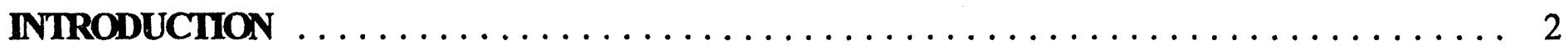

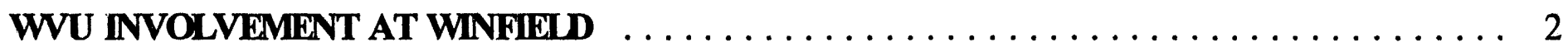

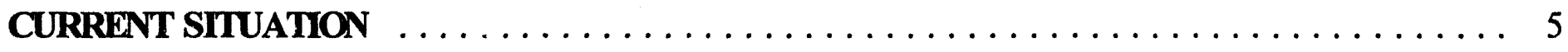

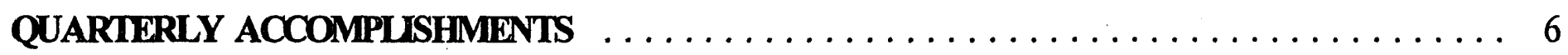




\section{EXECUTIVE SUMMARY}

The United States Army Corps of Engineers (ACE) must remediate a contaminated industrial site, formerly owned by ACF Industries, Inc., in order to build a new lock on the Kanawha River at Winfield, WV. The original remediation plan, to incinerate the contaminated soil, generated much controversy in the surrounding communities, notably Eleanor, WV.

West Virginia University became involved in the project in August of 1992 . Shortly after that time, a formal program to involve WVU in the solution to the remediation problem began. The WVU involvement has been two-pronged. WVU has interacted extensively with the citizens of Eleanor (and Putnam County) providing technical, logistical and financial assistance in their efforts to understand and evaluate the ACE plans for remediation. A primary objective for WVU is to inform the citizens of possible health and safety problems with proposed operations. In order to efficiently develop and communicate the necessary technical information, a resource group has been developed at WVU which is charged with developing enhanced technical (and other) capability to enhance the WVU effort. The members of this group have traveled, read and otherwise devoted some of their time to enhancing their knowledge of remediation. Some have provided insightful comments concerning published ACE plans and specifications (P\&S). Other WVU faculty have developed site opinion surveys and studied social, political and economic issues associated with community involvement in hazardous waste site remediation.

A second portion of the WVU effort is directed towards actually supplying assistance in development of a remediation strategy for the site. The ACE does not have a particularly clear idea of what to do if incineration is not used and they have requested help from WVU and other parties in investigating their options. Such assistance involves an expanded effort by WVU to investigate the state of the art of remediation and begin development of some new technologies. These investigations are being conducted by the National Research Center for Coal and Energy (NRCCE) staff and WVU faculty. 


\section{INTRODUCTION}

The site of the former ACF Industries, Inc. railroad tank car maintenance shop is contaminated by various organic and inorganic chemicals. The company abandoned the site in March 1986 and the U.S. Army Corps of Engineers (ACE) acquired the site, which was needed for the approach to expansion of the Winfield Locks and Dam. The extent of contamination, and most explicitly, the presence of dioxin at the site, had been inadequately documented before the ACE took possession of the land. The ACE, when it became aware of the extent of contamination, undertook a study which culminated in the publication of an Engineering Evaluation and Cost Analysis (EE/CA) in May 1992. The EE/CA was made public with a 30 day response period. The net conclusion of the EE/CA was that the ACE would incinerate the contaminated soil. The combination of citizen perception that they had not been appropriately informed of the problem and the proposal to incinerate the soil led to a concerted effort by the citizens to have the EE/CA reconsidered. The concerted effort, which included the involvement of the WV Congressional delegation, successfully led to reconsideration of the issue by the Army.

The subsequent reevaluation by the Army took nearly 7 months; the results, an Action Memorandum for the site, was released in December 1992. The Action Memorandum proposed that the soil be excavated, stored in temporary buildings, then remediated after further study.

As a result of the public involvement in the lock issue, Senator Robert C. Byrd of West Virginia and the Senate appropriations committee published some language which involved the Department of Energy and West Virginia University in the problem. The role of the DOE was to assist WVU financially in their efforts to safeguard the health and safety of the citizens and investigate the application of DOE technology in remediation of the site. The DOE-WVU effort is, thus, funded by this Cooperative Agreement and consists of establishment of a Resource Group and direct assistance to the area around the site as well as certain technological investigations directed towards the choice of the eventual remediation technology (or technologies).

\section{WVU INVOLVEMENT AT WINFIELD}

\section{Early Developments}

West Virginia University first became aware of Winfield and the problem existing there in August 1992. Representatives of WVU traveled to the site to discuss the problem with the ACE. The site was viewed from the outside and the history of the site and the nature of the lock expansion project were discussed.

In September 1992, the magnitude of concern for the remediation problem became more obvious. A representative of the NRCCE, Raymond J. Lovett, was chosen to become involved in the situation to provide technical and other assistance to the community. At the time, the concerned community consisted largely of an environmental group, P.R.O.T.E.C.T., and the town government of Eleanor. Eleanor contains three schools close to the site and Winfield has another three schools somewhat farther away, although Winfield is east of the site and more likely to be downwind.

On October 16, 1992, P.R.O.T.E.C.T. sponsored an appearance by Dr. Paul Connett, a chemist from Clarkson University in Potsdam, NY. Dr. Connett is an outspoken opponent of incineration, and organochlorine 
compounds, in any form. His talk was a, self proclaimed, polemic against incineration and the hazards of dioxin (which is actually used as a catchall descriptor of two classes of compounds, dioxins and furans, both of which are chlorinated). $\mathrm{He}$ in particular discussed the toxicity of 2,3,7,8 tetrachloro-p-dioxin, the most potent congener. Following Dr. connett's talk, contact was made with both the ACE and ACF Industries, which promised as much assistance as needed to help the WVU efforts. Discussions with the area citizens, including P.R.O.T.E.C.T. members and public officials revealed that although P.R.O.T.E.C.T. members were adamantly opposed to incineration, other community members and officials were willing to accept incineration if it were shown to be the best option.

On November 4, 1992, a trip was made to Eleanor to visit with concerned citizens and, the next day, with some state Department of Environmental Protection, Congressman Bob Wise's staff and Paul Hill from the National Institute for Chemical Studies (NICS). The visit to Eleanor consisted of talks with Marlene Carr, a town of Eleanor councilwoman, the mayor of Eleanor, Lloyd Jividen, and the P.R.O.T.E.C.T group. The P.R.O.T.E.C.T. group gave me a copy of the complete record of the site, all 17 volumes. That evening I dined with Missy Woolverton of the WV Citizen's Action Group (WVCAG); we discussed the situation at Winfield.

The discussion on November 5, 1992 with the WVDEP included Dave White and Lucy Pontiveros of the Office of Air Quality, Lewis Baker of the Office of Solid Waste and Ken Ellison, an assistant director of OSW. The conversation generally concerned the roles of WVU and the state in the remediation. As with most of these early discussions, the WVU role was ill-defined and the agencies were attempting to determine exactly the course WVU would take. Following the DEP talks, a meeting with Susan Small of Congressman Bob Wise's staff and Paul Hill of NICS took place. In this meeting, aside from trying to develop the role of WVU, Susan revealed a plan to form an umbrella group from citizens in the area to coordinate the local effort.

Shortly after the visit to Charleston, the ACE called and asked me to come to Huntington to discuss the role of WVU. The ACE notes of that meeting on November 16 and a subsequent meeting in Morgantown on December 15 provide information on the remediation and the relationship between the Corps and WVU. The relationship is one of assistance and cooperation, but with the maintenance of distinctly separate identities.

\section{The Winfield Lock and Dam Umbrella Committee}

The Umbrella Committee was formed in January 1993 and consists of politicians and citizens whose job included overseeing and evaluating ACE operations. Since its formation, the Umbrella Group has held meetings almost every second and fourth Tuesday of each month. They are divided into a number of committees, including health and safety, technology and project watch. Each meeting consists of a committee report followed by new business; sometimes the ACE is invited to explain its latest plans. The health and safety committee has concerned itself with airborne problems and the safety of school children. They have requested a local health assessment from WVU, negotiations on which are in progress. The technology committee reviews ACE plans and specifications and risk assessments. The project watch committee oversees activity at the site.

WVU interaction with the ACE has largely been devoted to definition of role and technical review of their plans. Among the plans published are the air monitoring plans, the storage building P\&S, and the demolition and decontamination P\&S and risk assessment documents. When published, the plans are submitted to the Umbrella Group and WVU. The plans are distributed to members of the Resource Group at WVU for evaluation. Those evaluations are distilled by the NRCCE and submitted to the ACE.

Interaction with political units has largely been confined to Eleanor. The Corps of Engineers is going 
to buy the Eleanor water supply, due to potential contamination from the site. WVU assisted the town (largely financially) in obtaining an independent valuation of their supply so that they could appropriately consider the ACE offer.

\section{Meeting with Army Corps of Engineers (Cincinnati, OH) (June 30 1993).}

The Army Corps of Engineers (ACE), in their quest to develop a suitable remediation scheme for the Winfield site, contacted the University of Cincinnati (UC) to discuss a possible role for them in the Winfield project. I was also invited to the meeting, held June 30, 1993 at the Center Hill facility in Cincinnati. In attendence were Jerry Roberto and larry Murdock (UC) and Gen. Albert Genetti and Richard Conner (ACE). Gen. Genetti was the commanding officer of the Ohio River Division and Richard Conner is the Chief of the Engineering Division at the Nashville ACE office, which is the Division center for environmental excellence.

The University of Cincinnati lised to operate the EPA Treatability facility at Center Hill (the contract has since been vacated). Although they performed lab tests to determine the suitablility of various remediation methods for various soils, their greatest efforts have been devoted to in-situ remediation. Specifically, UC has devoted much effort to hydrofracturing and steam enhanced vapor extraction, which they have tested on site. Such strategies are not particularly of interest at Winfield, since the soil will be excavated. The University of Cincinnati group also has a formidible effort in computer based site vizualization software, which is very impressive.

The meeting largely was informational, nothing was decided. I was however invited to visit Nashville, which will be discussed below.

\section{Umbrella Group}

A number of meetings were missed in the summer of 1993, due to trips or vacations taken by Group members or me. The Group elected a President (Tim McCoy) and adopted a set of by-laws. They discussed their vision for a health study and commented on the two ACE documents that were released this summer, a preliminary exposure scenario and a constituents of concern (COC) document. The group received a $\$ 25,000$ grant from Gov. Caperton's office to support a technical assistant, which they plan to use to hire Fred Youngs' Citizens for Jobs in the Environment group. Additionally, they appear poised to receive $\$ 100,000$ from the U.S. Congress to support another expert who will help develop remediation plans. These issues and others are discussed in the trip report for the one summer meeting I attended (August 24 ). There was no meeting held on August 10.

The August 24 meeting concerned the preliminary exposure scenario, which detailed the extent to which possible exposures would be assessed. The meeting was somewhat acrimonious, with most of the animosity directed towards the ACE subcontractor, Dames and Moore. The meeting was attended by Dr. Alan Ducatman of the WVU Department of Occupational and Environmental Medicine, who is savvy about risk assessments. The Dames and Moore Baseline risk assessment process is being broken down into parts to allow continuous input into the baseline risk assessment by the Umbrella Group. The Baseline risk assessment will detail the hazard of the site in the absence of remediation (i.e its intrinsic health risk). The constituent of concern portion used a series of criteria to limit the chemicals to be evaluated in the risk assessment. About 83 were chosen for further consideration, which is a large number as these sorts of analyses go. 


\section{Opinion Survey}

Last winter, Dr. Susan Hunter of Political Science at WVU was commissioned to conduct an opinion survey to determine the actual state of mind of the county citizens. The survey was initiated due to the general lack of knowledge concerning the thoughts of the vast majority of the citizens (although certain citizen thoughts had been widely broadcast through PROTECT and other groups).

\section{Health Survey}

The Umbrella Group has been concerned about how to evaluate the effects of the site and the cleanup. A number of conversations with Dr. Alan Ducatman were conducted over the last few months, which resulted in a decision by the Group to focus their energies on ensuring adequate monitoring at the site. Dr. Ducatman indicated that monitoring would be the best direct assurance that their health was not (or was) threatened. If an incident occurred, knowledge of the identity of the chemical would allow directed evaluation of exposed individuals, facilitating treatment. Lack of airborne exposure is the objective. The only other sort of evaluation that may make sense is a complete medical monitoring study, which would necessarily involve a large number of citizens ( $1 / 3$ or more). The medical surveillance would require periodic medical exams and long term evaluation of site proximity and other possible exposures. Dr. Ducatman predicted that the outcome would be negative (indicating no long term health effects), but assured the Umbrella Group that that was the only way to discern any health effects if they were to occur.

The Umbrella Group had initially wanted an epidemiological study, then possibly a health survey (oral), to determine the baseline health. Dr. Ducatman indicated that such a survey is biased at best and would not provide any useful information to protect health, much less indicate any long term effects. The argument that the area was already unhealthy (a possible finding of the survey) would provide no more convincing argument against emissions from the site than would a simply declaration that emissions should be minimized to prevent healthy people form becoming ill. In the end, the Group chose to concentrate on demanding appropriate monitoring (and, if necessary, appropriate response to exposure incidents).

\section{CURRENT SITUATION}

\section{Lock and Dam}

The Corps of Engineers began excavating for the lock in the spring of 1993. Only noncontaminated areas have been excavated, downstream of the ACF site. The Corps has released the preliminary risk assessment for the removal action (dismantling and excavation) at the site. Current plans are for excavation (preceded by dismantling and decontamination of the buildings) to begin in November 1994 and be completed in December 1995. The plans and specifications for the removal action are not yet available. The bids for the temporary storage buildings have been opened and the buildings are supposed to be ready by next November. A problem has arisen, two bids were close and both were technically the low bid (the problem revo!ves around the as yet undecided upon issue of the scrubber). At last report, the Attorney General has yet to rule on which bid can be accepted. The Corps has until February to decide whether a scrubber will be added to the building. The entire construction project will be finished by January 1997, but remediation of the soil will take longer (in fact no completion date is postulated for the end of the 
remediation).

\section{Umbrella Group}

The Umbrella Group now has officers, but attendence has been declining. They are poised to hire an expert for review of future Corps plans and are largely concerned now with review of Corps risk assessment documents. Of critical concern in the months ahead will be the plans for excavation and monitoring; there has been little action on site since the sampling by ACF and Dames and Moore last spring.

\section{QUARTERLY ACCOMPLISHMENTS}

\section{Umbrella Committee Meetings}

Umbrella Committee Meeting of September 14, 1993.

Cancelled

Umbrella Committee Meeting of September 28, 1993.

I was in Atlanta, GA at a meeting on Emerging Technologies in Hazardous Waste Treatment.

Umbrella Committee Meeting of October 12, 1993.

The meeting was held at the Eleanor Town Hall at 7:00 P.M., I also attended the Technology and Health committees meetings starting at 6:00 P.M. The Corps of Engineers arrived at 8:00 P.M. Only the Huntington representatives were there.

At the technology committee meeting, I reported or a visit to Clean Sites in Alexandria, VA. I had originally been apprised of Clean Sites by Mark Kessinger of the Huntington COE. I had encountered Sarah Medearis of Clean Sites in Atlanta at the ACS Emerging Technologies meeting and arranged a visit (on 107). The Umbrella Group wanted to know how Clean Sites felt they would interact with the Winfield Project. Based on my conversations at Clean Sites I reported that their involvement, if any, would largely lie in the technology decision stage, not at any earlier stage. No plans exist at all for the involvement of Clean Sites at this time.

I also discussed my plans to visit the Nashville, TN COE office with the Group. In particular I inquired if they would like treatability studies conducted at the site; they were enthusiastic. My trip to Nashville (10-19) revealed that the COE is eager for such tests as well and that Nashville will evaluate proposed tests, but Col. Richardson in Huntington will make the eventual decisions.

The Health and Safety committee discussed the meeting they had with Dr. Alan Ducatman and I (and others) at WVU on October 6. Based on those discussions, they voted to concentrate their efforts on assuring that adequate monitoring is installed when site work begins. These committee meetings occupied the normal meeting time as well. The subjects shifted when the Corps arrived.

The Corps will help with the school shelter in place program and the Group and the COE discussed some details. It was noted that the scrubber issue must be decided by February, in order to activate the option on it. A discussion on Environmental impact statements ensued, Mark Kessinger subsequently sent 
me copies. The Corps contractor, Dames and Moore, will show up on November 9 [actually November 30] to discuss new documents they have prepared. Current documents are the Constituents of Concern, Exposure Assessment and Air Monitoring Study. Discussion of the constituent of concern document was directed mostly towards me. I indicated that the criteria used appeared sensible and that every class of compound was still represented on the list (of 83). The Umbrella Group appeared confortable with the list, but will reserve their comments until Dames and Moore appear. Since Dames and Moore are contractors of Nashville, the Group recognizes that comments directed to Huntington are not as effective as comments to Dames and Moore directly or representatives from Nashville.

Umbrella Committee Meeting of October 26, 1993.

Cancelled

Umbrella Committee Meeting of November 9. 1993.

Cancelled

Umbrella Committee Meeting of November 30, 1993.

The Corps of Engineers and Dames and Moore attended the meeting. The major topics of discussion were the interim risk assessments. The initial discussion concerned the oral report presented by ACF in August and their follow-up in September. The ACF conclusion that large amounts of soil from the lock site could be disposed of in landfills was disputed by the Corps. Landfills contacted by the Corps said that they would be prohibited from accepting dioxin contaminated soil by, generally, state regulations

The discussion of the Dames and Moore documents largely centered on whether the risk is adequately evaluated. Once again the Umbrella Committee expressed concern over the absence of specific consideration of school children in the documents. D\&M explained that children are implicitly considered; the Committee wanted more definite consideration. The risk analysis used EPA models, looked at average values not pulse releases of hazardous materials. The air dispersion process was discussed. The results lead to the estimated exposure with distance. The Umbrella Committee cannot dispute the models until they can hire their expert.

Umbrella Committee Meeting of December 14, 1993.

Missed

Umbrella Committee Meeting of December 28, 1993.

No meeting scheduled.

Corps of Engineers Meetings

Nashville Office Meeting of October 19, 1993. 
On October 19, 1993 I visited the Corps of Engineers office in Nashville, TN. The purpose of the vist was to generally discuss the Winfield situation and determine what would be necessary for WVU to initiate technology evaluations at the site. After general discussion, I learned that any plans for evaluating a technology at the site would first involve review by the Nashville Office (which is the Division Center of Excellence for Environmental Remediation). The recommendations of Nashville would be considered by Col. Richardson in Huntington, who would make the final decision. The initial application would be written and the technology would require a fairly high level of test data to show feasibility. The written request should also include space requirements and detailed plans on managing and executing the feasibility study.

\section{Visit to Waterways Experiment Station. Vicksburg. MS on November 23, 1993.}

\section{Representatives of the Huntington Corps, WVU and the Morgantown Energy Technology Center} visited the Waterways Experiment Station in Vicksburg. This site is where most Corps of Engineers research in hazardous waste remediation is conducted. The vist began with a discussion of the capabilities, followed by a tour of the facilities. Most of the work at Vicksburg concerns ex-situ treatment methods. Technologies included substantial efforts in bioremediation (much in reactors), an ultraviolet pump and treat system, a Roy F. Weston thermal desorber unit, and some early work with electrokinetics. They discussed previous work with chemical dechlorination as well.

Subsequent tours displayed the Ecological Risk assessment research program for dioxin and a roundtable discussion of the issues involving Winfield that must be answered.

\section{Other Meetings}

\section{Town Meeting in Eleanor, WV on November 2, 1993.}

This meeting was held by AE Environmental to explain the results of the ACF confirmatory sampling program. In general, AE agreed wiith most of the ACF conclusions. The most controversial conclusion was that the site contained not 60,000 cubic yards of contaminated material, but 5-10,000 cubic yards. ACF concluded that the rest of the allegedly contaminated material could be landfilled [this is disputed by the Corps]. AE recommended that a new, more statistically valid, sampling program be initiated at the site. The ACF study focussed on areas that the Corps found to be contaminated; the study did not evaluate areas which had been declared free of contamination. In effect, ACF attempted to assert whether the Corps had erred in their positive analyses, but did not make any attempt to determine whether the Corps had erred in their negative analyses. AE recognized this flaw. 
Assessment of Technologies for Hazardous Waste Site Remediation: NonTreatment Technologies and Pilot Scale Test Facility Implementation (Project MC-10)

Quarterly Report for Period

for Period October 1 through December 31, 1993

Work Performed Under Contract

No.: DE-FC21-92MC29467

For

U.S. Department of Energy

Office of Fossil Energy

Morgantown Energy Technology Center

Morgantown, West Virginia

By

Harry R. Johnson

BDM Federal, Inc.

1199 Van Voorhis Road, Suite 4

Morgantown, West Virginia 26505

February 1994 


\section{QUARTERLY REPORT}

\section{EXECUTIVE SUMMARY}

Projects one two and three were completed at the end of the last quarter and the first draft report submitted for technical review. Project one determined that extensive research on storage technoiogy, particularly with temporary or permanent buildings, has not been conducted. There are few researchers who are actively examining some aspect of storage technology. Those identified are involved in nuclear waste storage technology. This report is being finalized for submission to the project office.

Project two examined the most recent developments in excavation technology for the Winfield site, a long arm track hoe was found to have several advantages over other types of excavating equipment. Dust suppression using chemicals is a problem since the chemical needs to be tailored to the specific soils being excavated. Additional research work in this area is needed.

Project three examined the potential application of a large number of monitoring equipment that would be applicable to the Winfield site. The results of our study indicated that a combination of Gas Chromatigraph and Mass Spectrometer used in conjunction with a Fouier Transform Infrared Spectrometer would provide the best instrument package for use at the Site.

Project four examined the potential uses and projects that might be conducted in METC Building B-17. BDM examined the requirements for bench scale and slightly larger, pilot-scale fluid flow experiments. Pressure vessels to contain such experiments were examined and experiment flow diagrams prepared to support the development of a SARS package for the potential project. BDM concluded that it is almost impossible to complete a SARS package unless all planning has been completed because of the level of detail required for the safety analysis. BDM subcontracted with the WVU extension office's Institute for Safety and Health Training to develop the SARS for this project as training for supporting future $R$ \& $D$ experiments that may be conducted in Building $B$ 17. 


\section{PROJECT 1 Assessment of Environmental Remediation Storage}

\section{Technologies}

Project one concluded that extensive research on storage technology particularly with temporary or permanent buildings, has not been conducted. There are few researchers who can be identified as actively examining some aspect of storage technology.

Final preparations of the draft report were made prior to submittal of the final report on December 30, 1993.

\section{PROJECT 2 Assessment of Environmental Remediation Excavation}

\section{Technologies}

During the last quarter, the most recent developments in excavation technology were examined for the Winfield site, a long arm track hoe had several advantages over other types of excavating equipment. Dust suppression using chemicals is a problem since the chemical has to be tailored to the specific soils being excavated. Additional research work in this area is needed.

Final preparations of the draft report were made prior to submittal of the final report on December 30, 1993.

\section{PROJECT 3 Assessment of Environmental Remediation Monitoring}

\section{Technologies}

During the last quarter, we examined potential application of a large number of monitoring equipment that would be applicable to the Winfield site. The results of our study indicated that a combination of Gas Chromatigraph and Mass Spectrometer used in conjunction with a Fouier Transform Infrared Spectrometer would provide the best instrument package for use at the Site.

Final preparations of the draft report were made prior to submittal of the final report on December 30, 1993. 
PROJECT 4 Assessment of Research Projects Planned for Implementation in the B-17 Building

The objectives of this task include (1) the identification of projects for implementation in Building B-17 at METC, (2) review/develop project plans, (3) conduct safety analysis and reviews (SARS) in accordance with DOE orders for projects identified, and (4) preparation of a project assessment report.

\section{Project Identification}

No funded projects currently exist for Building B-17. In lieu of funded projects, the project proposed under the METC/DOE Research Opportunity Announcement (ROA) to develop test protocols for barrier materials was selected as the most likely candidate for near-term funding and as the candidate for development of a "SARS package".

\section{Review and/or Development of Project Plans}

Because of the size, complexity, and minimal test procedure definition of the candidate project, we elected to concentrate our efforts on the one experiment described in the proposal. This experiment proposes the formation and evaluation of pilot-scale insitu barriers by creating a $20^{\prime} \times 4^{\prime} \times 10^{\prime}$ test bed inside of a rectangular vessel, injecting a barrier material through an injection well near the center of the test bed, and determining the fluid conductivity of the system using injection and sampling ports at opposite ends of the vessel. (See Figure 1 - Original Proposed Apparatus and Procedures for Testing Barrier Materials). This experiment was subsequently improved as next described.

Upon analysis of the proposed apparatus and procedures, a number of questions arose which led to the conclusion that certain preliminary tests should be conducted prior to the "pilot-scale" test in the large vessel. These questions centered on the issues of realistic design pressures, evaluation and control of boundary effects at the walls of the vessel, and best use of data from a test in a large vessel. An alternative apparatus and test plan was generated with the major objectives being to acquire data which would help answer some of the questions raised and to provide information similar to that sought in the original experimental plan. A pressure vessel was designed which would allow the creation/simulation of "insitu" barriers. This vessel will consist of a 10-ft., large diameter (18 in.) pipe with a Hassler-type sleeve (approximately 12 in. diameter) 
inside the vessel. The vessel will allow barrier injection and/or fluid flow tests to be conducted safely under a wide range of reasonable pressures as well as providing reasonable access to the barrier materials for post-test analysis. Procedures for utilizing the apparatus have been developed as have procedures for pre- and post-test evaluation of basis parameters such as porosity and permeability.

\section{Safety Analysis and Reviow}

To properly train University personnel in the process of SARS preparation, BDM, with approval of the WVU project manager, began working with WVU Extension Service's Institute for Safety and Health Training to develop the SARS package for presentation to DOE/METC Progress to date includes two meetings this quarter with Mr. Tom Stockdale of the Extension Service to discuss the best approach to achieve the objectives of this subtask. The Extension Service will compile information on DOE/METC safety policies and procedures, determine which policies and procedures are applicable to the proposed experiment(s), and assemble the SARS package for presentation to DOEMETC for consideration.

Several items of significance, including some preconceptions and misconceptions, have been revealed in analyzing the SARS process as implemented at DOE/METC. These are as follows:

1. The DOE Order 5481.1B is the governing order, but is not adequate in itself to determine the content of a SARS package (for somewhat obvious reasons). Each DOE facility has location-specific considerations and has developed their own policies and procedures to implement the Order. DOE/METC is no exception.

2. The SARS package (at DOE/METC) is not a "one-time-submittal" package. The package is submitted in two separate but related packages. First, a "construction permit" application with associated SARS data is submitted. Upon approval and actual construction, an "operating permit" application with associated SARS data is submitted. In each case, the review and approval process is likely to consist of one or more iterations and resubmittals to the DOE/METC safety committees.

3. The SARS package is an integral part of the program activity/facility: i.e., a modified SARS package is prepared with each significant change in the operating procedures or the facility and the package is updated and reviewed annually to assure that no significant changes have been overlooked. 
4. Some SARS package requirements may be difficult for WVU to meet due to the nature of DOE/METC's SARS procedures. For example, the SARS procedures are written based on the premise of the work being done or directed by DOE/METC personnel and using permanent, onsite staff, who must be identified by name in the SARS package. This may pose occasional to frequent problems for WVU in being able to identify specific graduate students to be assigned to a project at the time a SARS package is being developed (ideally several weeks or months before a project is actually implemented). 
Purmose: The purpose of this apparatus is to form and evaluate pilot scal insitu barriers. The primany variable to be measured is the permeability of the barrier to aqueous solutions.

Experimental Schematic: A schematic of the apparatus is shown in Flgure 1.

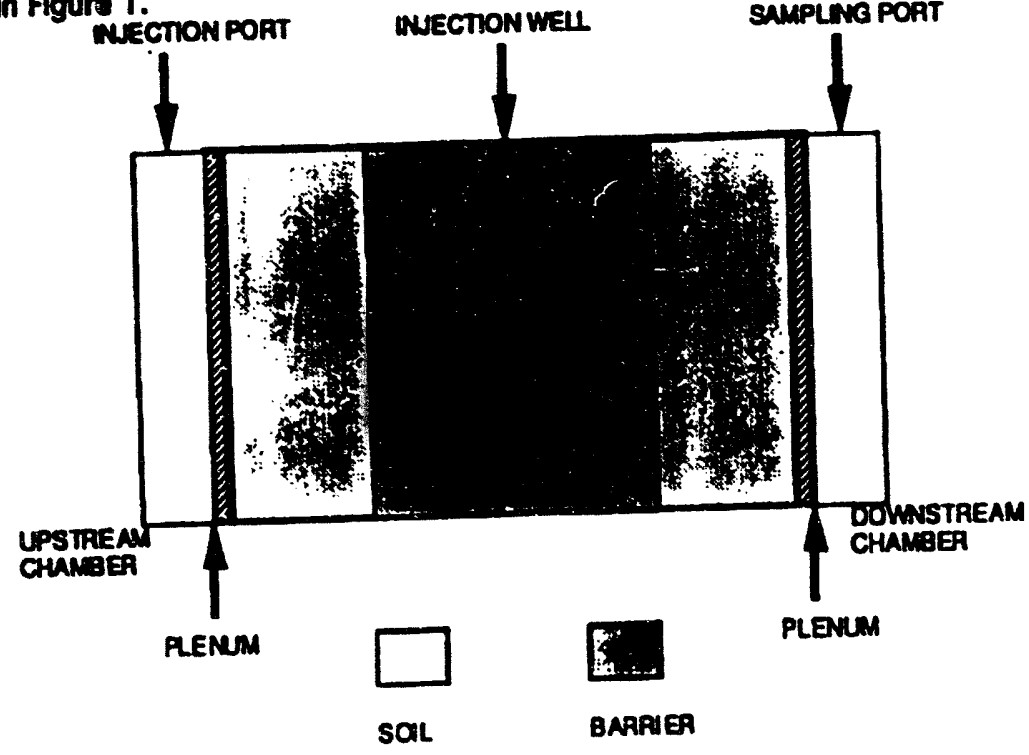

Figure 1

The experimental apparatus is a rectangular vessel nominally 20 feet long 4 feet wide and 10 feet high. A 12 inch pipe is located in feet long, 4 foet wida and The pipe is to serve as an injection well the center of tho vessel. Injection and sampling ports are located at for the barrier material. Injection and samping ports are located at the ends of the vessel. Aqueous solution is pressurtzed and expo to the barrier through the injection port with flow through the barrier being monitored

to operate at 200 psig.

Test procedure: The vessel bed is packed with sand and soil material to simulate various soil and rock conditions. The packed matis then irrigated with aqueous brine to obtain the desired water

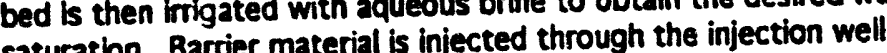
saturation. Barrer material is then allowed to at pressures up to 200 psig. The barrier mas to cured, an aqueous solution is injected through the injection port and pressurized up to 200 psig. The rate at which the aqueous solution flows through the barrier is monitored at the sampling port.

Packed Bed Characterization: The composition of the bed is determined with regard to sand, clay, pebble, etc. composition. After the desired overburden pressure is applied to the bed the porosity of the bed is measured. The permeability of the bed to the aqueous solution is measured. Pressure drop through the bed is measured. Brine saturation is determined and adjusted to desired value.

Aqueous Solution Characterization: The chemical composition, viscosity and density of the aqueous solution and brine are determined.

Barrier Material Characterization: The viscosity and density of the barrier material are determined.

Operational Temperature: The bed operates at temperatures from $55 \mathrm{~F}$ to $120 \mathrm{~F}$.

Monitored Variables: The injection pressure and flow rate of the aqueous solution through the barrier are monitored.

Post Flow Test Bed Characterization: After the pressure test has been completed, the bed is depressurized and opened. The bed is then cored at 6 inch increments to determine the distribution of barrier material in the bed. 


\section{WEST VIRGINIA UNIVERSITY COOPERATIVE AGREEMENT}
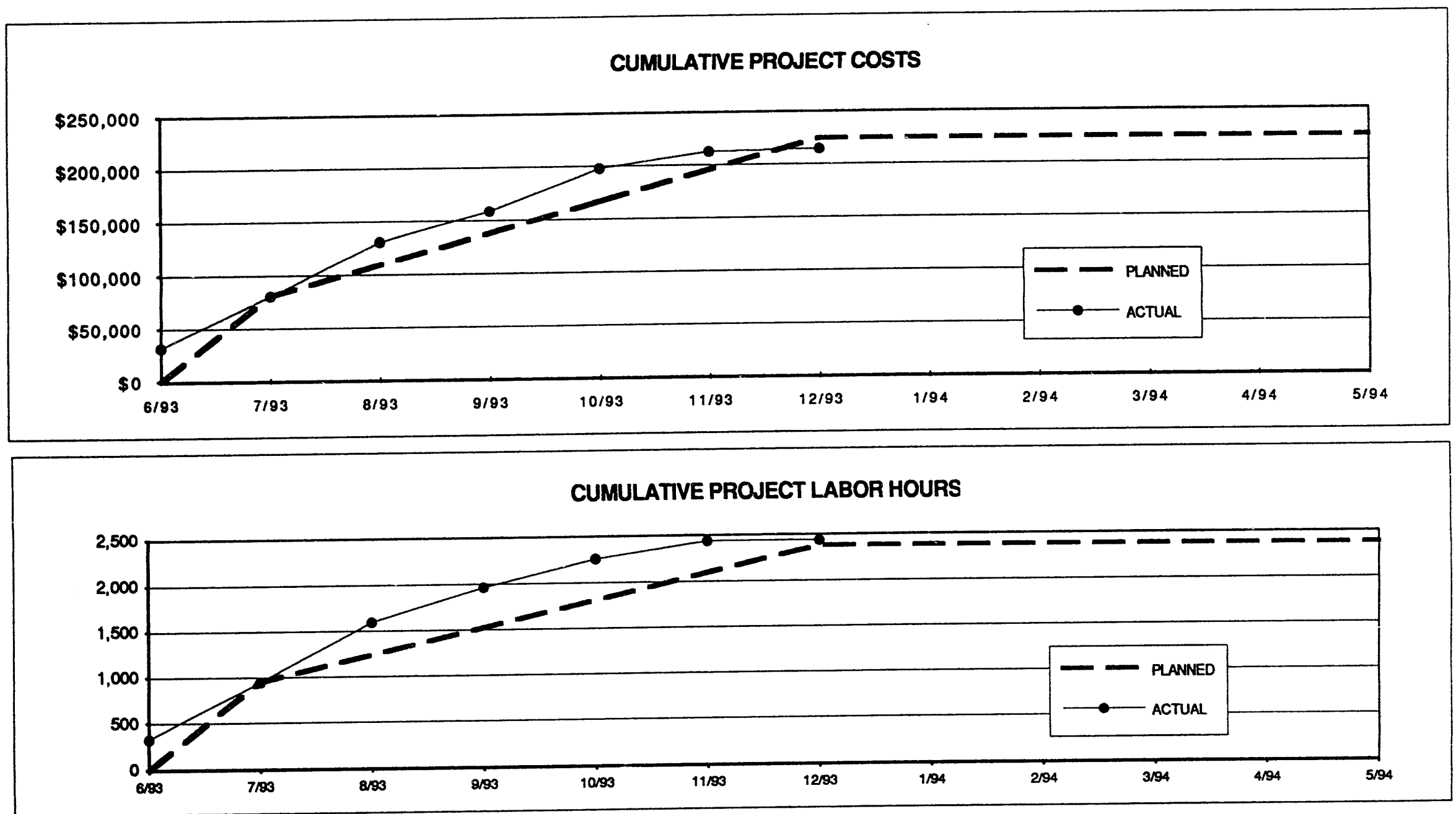


\section{WEST VIRGINIA UNIVERSITY COOPERATIVE AGREEMENT}

\begin{tabular}{|c|c|c|c|c|c|c|c|c|c|c|}
\hline \multirow[b]{3}{*}{ MONTH } & \multicolumn{4}{|c|}{ PLANNED PROJECT LABOR AND COSTS } & \multicolumn{6}{|c|}{ ACTUAL PROJECT LABOR AND COSTS } \\
\hline & \multicolumn{2}{|c|}{ LABOR HOURS } & \multicolumn{2}{|c|}{ TOTAL COSTS } & \multicolumn{2}{|c|}{ LABOR HOURS } & \multicolumn{4}{|c|}{ TOTAL COSTS } \\
\hline & MONTHLY & aM & MONTHLY & am & MONTHLY & $a_{M}$ & MONTHLY & cam & RESERVE & Remaining \\
\hline 6/93 & 0 & 0 & $\$ 0$ & $\$ 0$ & 336 & 336 & $\$ 31,916$ & $\$ 31,916$ & $\$ 7,318$ & $\$ 184,911$ \\
\hline $7 / 93$ & 953 & 953 & $\$ 81,105$ & $\$ 81,105$ & 617 & 953 & $\$ 49,189$ & $\$ 81,105$ & $\$ 7,318$ & $\$ 135,722$ \\
\hline 8/93 & 285 & 1,238 & $\$ 28,608$ & $\$ 109,713$ & 646 & 1,599 & $\$ 50,249$ & $\$ 131,354$ & $\$ 7,318$ & $\$ 85,473$ \\
\hline 9/93 & 285 & 1,523 & $\$ 28,608$ & $\$ 138,321$ & 370 & 1,969 & $\$ 27,848$ & $\$ 159,202$ & $\$ 7,318$ & $\$ 57,625$ \\
\hline $10 / 93$ & 285 & 1,808 & $\$ 28,608$ & $\$ 166,929$ & 290 & 2,259 & $\$ 39,238$ & $\$ 198,440$ & $\$ 5,146$ & $\$ 20,559$ \\
\hline $11 / 93$ & 285 & 2,093 & $\$ 28,608$ & $\$ 195,537$ & 176 & 2,435 & $\$ 14,473$ & $\$ 212,913$ & $\$ 2,748$ & $\$ 8,484$ \\
\hline $12 / 93$ & 285 & 2,378 & $\$ 28,608$ & $\$ 224,145$ & 이 & 2,435 & $\$ 1,894$ & $\$ 214,807$ & $\$ 2,061$ & $\$ 7,277$ \\
\hline $1 / 94$ & 0 & 2,378 & $\$ 0$ & $\$ 224,145$ & & & & & & \\
\hline $2 / 94$ & 0 & 2,378 & $\$ 0$ & $\$ 224,145$ & & & & & & \\
\hline $3 / 94$ & 0 & 2,378 & $\$ 0$ & $\$ 224,145$ & & & & & & \\
\hline $\begin{array}{r}4 / 94 \\
5 / 94\end{array}$ & $\begin{array}{l}0 \\
0\end{array}$ & 2,378 & $\$ 0$ & $\$ 224,145$ & & & & & & \\
\hline 5/94 & 0 & 2,378 & $\$ 0$ & $\$ 224,145$ & & & & & & \\
\hline
\end{tabular}


Remediation of Hazardous Sites with Steam Reforming (Project MC-11)

Quarterly Report for Period

for Period October 1 through December 31, 1993

Work Performed Under Contract

No.: DE-FC21-92MC29467

For

U.S. Department of Energy

Office of Fossil Energy

Morgantown Energy Technology Center

Morgantown, West Virginia

By

Babak AghaMohammadi

Manufacturing and Technology Conversion

International, Inc. (MTCI)

Columbia, Maryland 21044 


\begin{abstract}
Fabrication of the pressurized Process Development Unit is progressing. The reactor shell has been completed including the modifications required to accommodate a classifier for controlling fluid-bed particle size. A second cyclone has been designed with a refractory lining to recycle the captured solids to the reactor. The pulse tube heater bundle design has also been modified to eliminate dead spaces that could result in tube temperature overheating.
\end{abstract}




\section{TABLE OF CONTENTS}

Section No.

Page No.

1. TECHNICAL BACKGROUND $\ldots \ldots \ldots \ldots \ldots \ldots \ldots \ldots$

2. EXPERIMENTAL METHODOLOGY $\ldots \ldots \ldots \ldots \ldots \ldots$

3. EXPERIMENTAL RESULTS AND DISCUSSIONS $\ldots \ldots \ldots \ldots$

4. CONCLUSIONS $\ldots \ldots \ldots \ldots \ldots \ldots \ldots \ldots \ldots \ldots \ldots \ldots$ 


\section{LIST OF FIGURES}

Figure

Page No.

Figure 1. Modified Pressurized Reactor Shell -

Bottom Section ...................... 4

Figure 2. Reactor Shell - Top Section $\ldots \ldots \ldots \ldots$

Figure 3. Schematic of the Classifier Assembly $\ldots \ldots \ldots \ldots$

Figure 4. Modified Process Flow Diagram . . . . . . . . . . . . 7

Figure 5. Pulse Heater Tube Bundle Configuration $\ldots \ldots \ldots \ldots$ 


\section{TECHNICAL BACKGROUND}

A complete discussion of the technical background was presented in this quarterly report for the period July 1 through September 30, 1993. For continuity of the discussion in this part, the following paragraph has been included herein.

The MTCI thermochemical reactor system has easily demonstrated at commercial levels its superior performance in the steam reforming of a wide variety of biomass, industrial and municipal waste feedstocks containing chlororganics as well as coal, coal liquefaction bottoms, and mild gasification char at ambient (atmospheric) conditions. The system processed a wide spectrum of feedstocks producing a hydrogen-rich product gas that is free of the diluents (primarily $\mathrm{N}_{2}$ ) found in air-blown steam reforming systems and is more cost-effective than oxygen-blown systems. In addition, liquid and solid waste streams from the MTCI system show extremely low levels of toxics and leachability, permitting easier landfill or industrial toxic byproduct recovery. The feasibility of developing a pressurized system based on the MTCI technology would permit even greater gains in processing yields, system modularity, and capital and operating costs for processing hazardous and other waste. This would especially be applicable to the cleanup and treatment of large volumes of soils contaminated with toxic organics, i.e., PCB's, pesticides, cyanides, RCRA metals, and dioxin/furans. Reformation of toxic organics in the oxidizing environment of incinerators are completely eliminated because of the reducing environment present in the MTCI thermochemical reactor (steam reformer). 


\section{EXPERIMLNTAL METHODOLOGY}

A proposed test plan and methodology was also included in the previous quarterly report. In addition to that material, MTCI is pursuing a discussion vith Radian, Inc. to provide independent sampling and analysis of all waste streams from the system during the experimental effort. 


\section{EXPERIMENTAL RESULTS AND DISCUSSIONS}

The fabrication of the PDU is progressing. The reactor shell, both top and bottom sections, are 100 percent completed and ready to be refractory-lined.

The reactor shell design was modified slightly to accommodate a classifier. These new changes are shown in Figure 1 and Figure 2.

Figure 1 shows the bottom section of the reactor. The bed overflow pipe was changed from 3" SS204 pipe to a 6" refractory line. Figure 2 shows the top section of the reactor. A 4" refractory pipe was added to the top section of the reactor. This new port is the classifier outlet port to the gasifier. It is anticipated that during long duration tests the average bed size will grow from 150 microns to approximately 250 microns. The smaller the bed particles the higher the fluidized bed heat transfer coefficient. Therefore, the fluid bed requires a "bed size management system" to keep the average bed particles in the desired range.

A classifier can be used to control the fluid-bed particle size. As shown in Figure 3, coarse particles can be drawn from the bottom of the bed and conveyed to the classifier using steam as the conveying medium. The classifier is a low-efficiency cyclone that captures only the coarse particles. The fine particles along with steam will be returned to the gasifier. The coarse particles will be collected in the catch pot where they will be crushed to smaller size and returned to the gasifier. By managing the bed particle size within the desired rarige, the fluidbed heat transfer coefficiency can be optimized and hence the overall system efficiency.

Another change to the process is to make the second cyclone a hot refractory-lined cyclone to return the captured solid to the reactor. In the previous quarterly report cyclone $\# 2$ was located after the waste heat recovery boiler (E-1). It was decided to recycle back the solids to the reactor to increase solid residence time; hence, the cyclone was moved back before E-1. Since the gas temperature and pressure will be approximately $1400^{\circ} \mathrm{F}$ and 5 atmospheres, the cyclone needs to be refractory-lined (Figure 4).

The pulse tube heater bundle configurations were also changed (Figure 5). In the previous configuration, the tubes were straight with 180-degree bends, forming peaks and troughs to give the desired surface area. It is anticipated that in this trough the bed media will settle and not fluidize and overheat the pipe and cause tube failure. The present configuration eliminates this problem. 

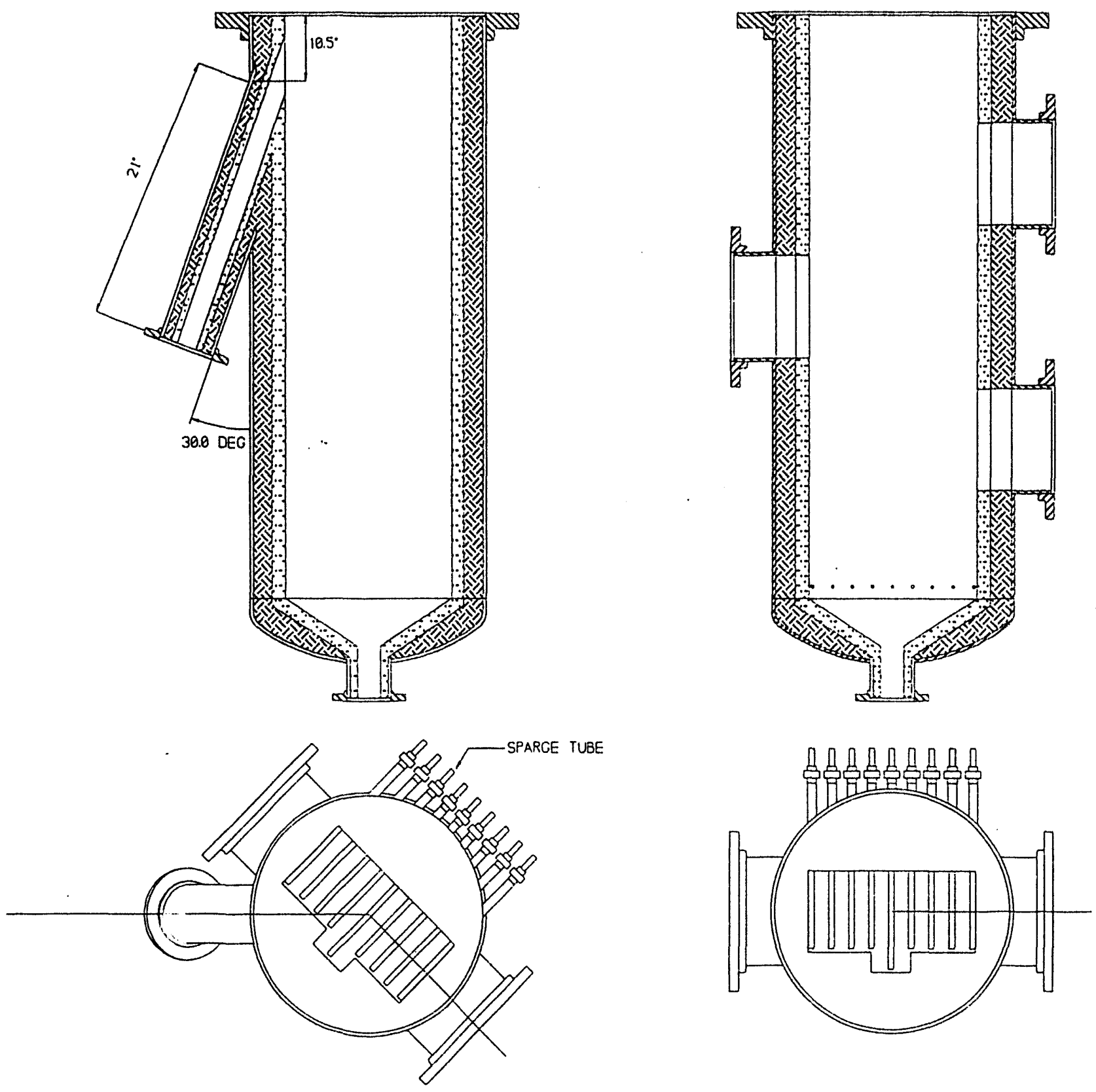

FIGURE 1: MODIFIED PRESSURIZED REACTOR SHELL BOTTOM SECTION 


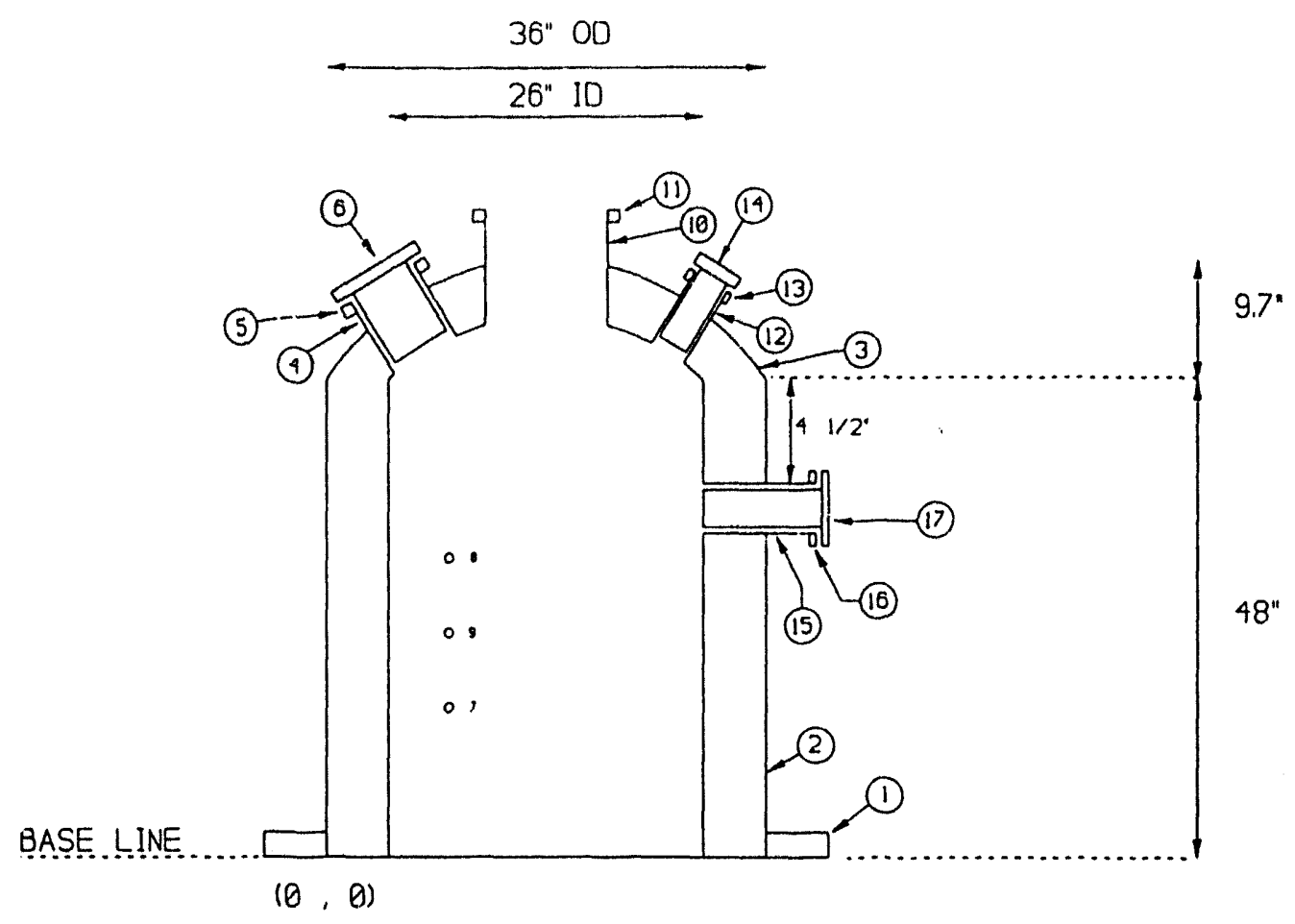

\begin{tabular}{|c|c|c|c|}
\hline ITEMS & DESCRIPTION & $\begin{array}{l}\text { ELEVATTON } \\
\text { FROM IERO } \\
\end{array}$ & DEGREES \\
\hline 1 & 36 , 150H, CS, SOF, RF & N/A & N/A \\
\hline 2 & $36^{\circ} \mathrm{OD}, 1 / 2^{\circ}$ WALL, CS, PIPE & N/A & N/A \\
\hline 3 & $36^{\circ} O D, 1 / 2^{\prime}$ WALL, H]-CROWN TANK HEAD & N/A & $N / A$ \\
\hline 4 & 6., CS, SCH 40. PIPE & $N / A$ & N/A \\
\hline 5 & $6^{\circ}, \mathrm{CS}, \mathrm{SOF}, \mathrm{RF}, 150 \mathrm{H}$ & N/A & N/A \\
\hline 6 & 6', CS, BF, RF, 158\# WITH PLUG & $N / A$ & N/A \\
\hline 7 & TC PORT, 3/4\%, CS, HALF COUPLINC THREADED & $12^{\circ}$ & 315 \\
\hline 8 & TC PORT, 3/4: CS, HALF COUPLINC THREADED & $24^{\circ}$ & 315 \\
\hline 9 & PRESSURE PORT, $1 / 2 *$ CS, HALF COUPLING THREADED & $18^{\circ}$ & 315 \\
\hline 10 & CYCLONE EXIT PORT, 10', CS. SCH 40, PIPE. 8' LONG & N/A & N/A \\
\hline 11 & 10: CS, SOF, RF, 150\# & N/A & $N / A$ \\
\hline 12 & 3', CS, SCH40, PIPE & N/A & N/A \\
\hline 13 & $3^{\circ}, \mathrm{CS}$, SOF, 150 & N/A & $N / A$ \\
\hline 14 & 3', CS, BF. 150= WITH PLUC & N/A & $N / A$ \\
\hline 15 & $4^{\circ}$. CS. SCH 40. STO PIPE & $41.5^{\circ}$ & 225 \\
\hline 16 & 4., CS, 150\#, STO, SOF & $N / A$ & N/A \\
\hline 17 & $4^{\circ}$, CS. BF, 150\# WITH PLUC & N/A & N/A \\
\hline
\end{tabular}

FIGURE 2: REACTOR SHELL - TOP SECTION 


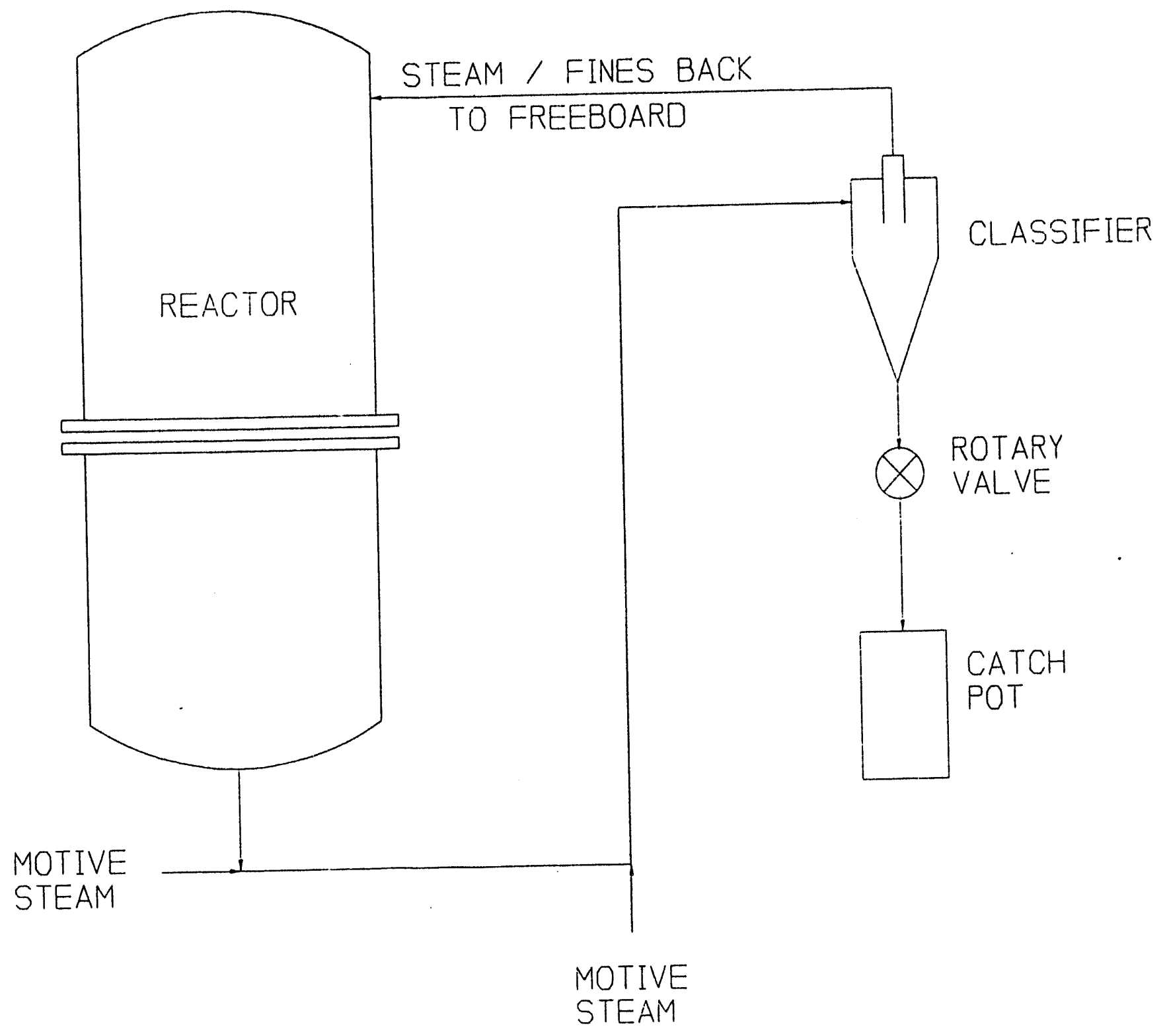

FIGURE 3: SCHEMATIC OF THE CLASSIFIER ASSEMBLY 

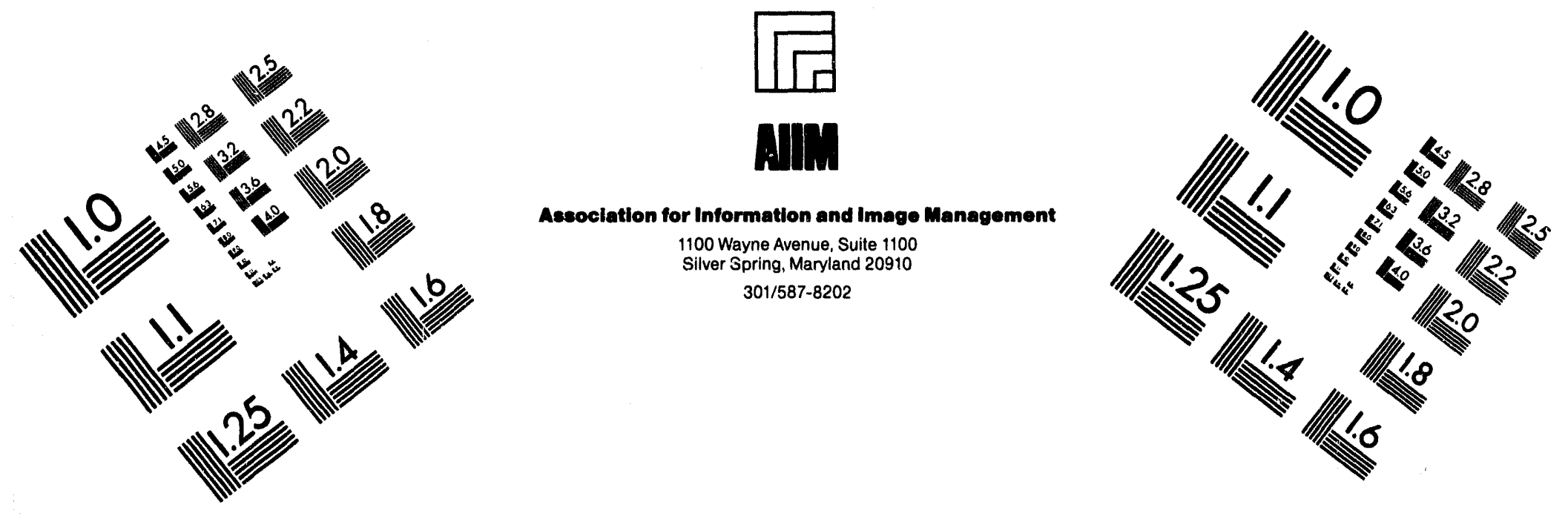

\section{Centimeter}

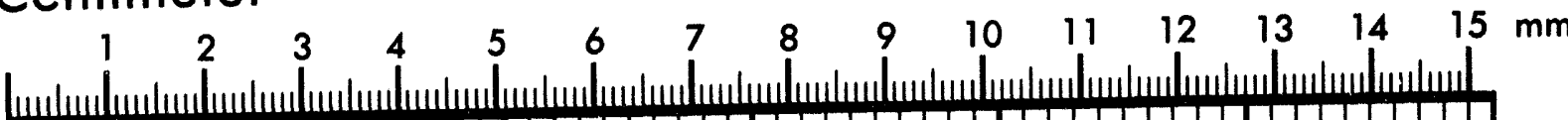

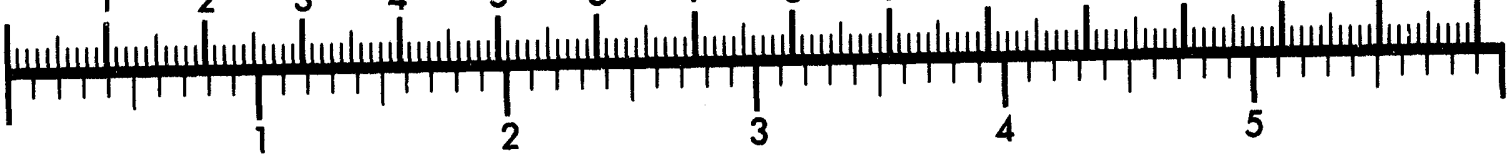
Inches

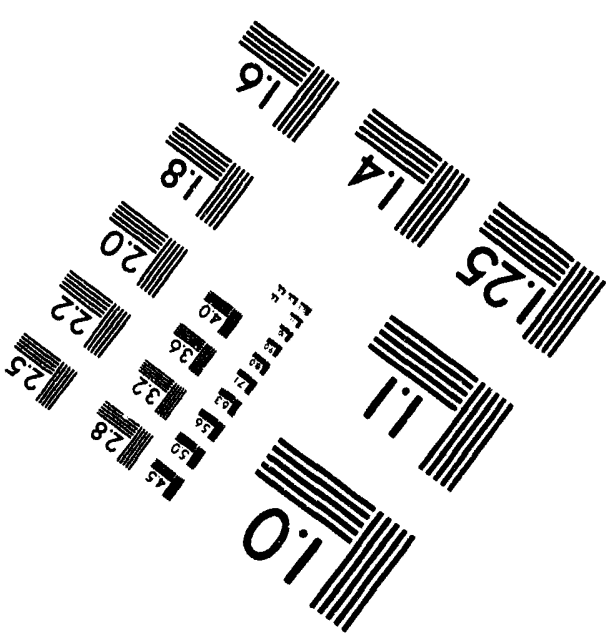

MANUFACTURED TO AIIM STANDARDS

BY APPLIED IMAGE, INC.

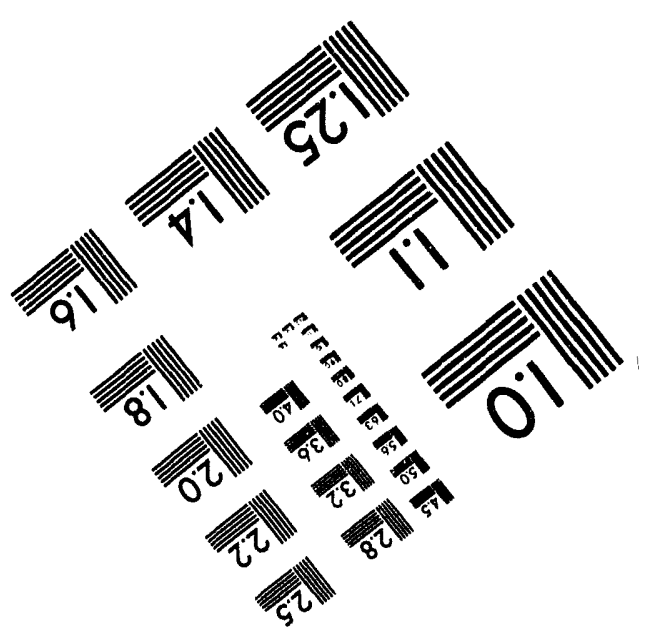



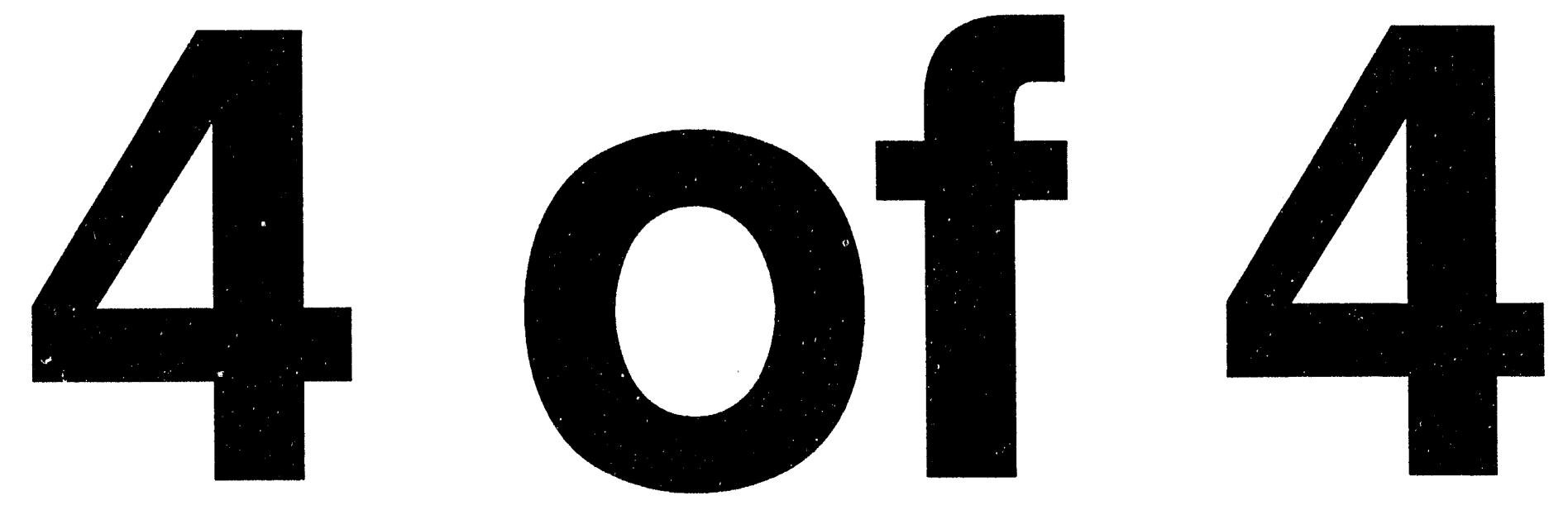

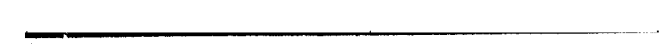




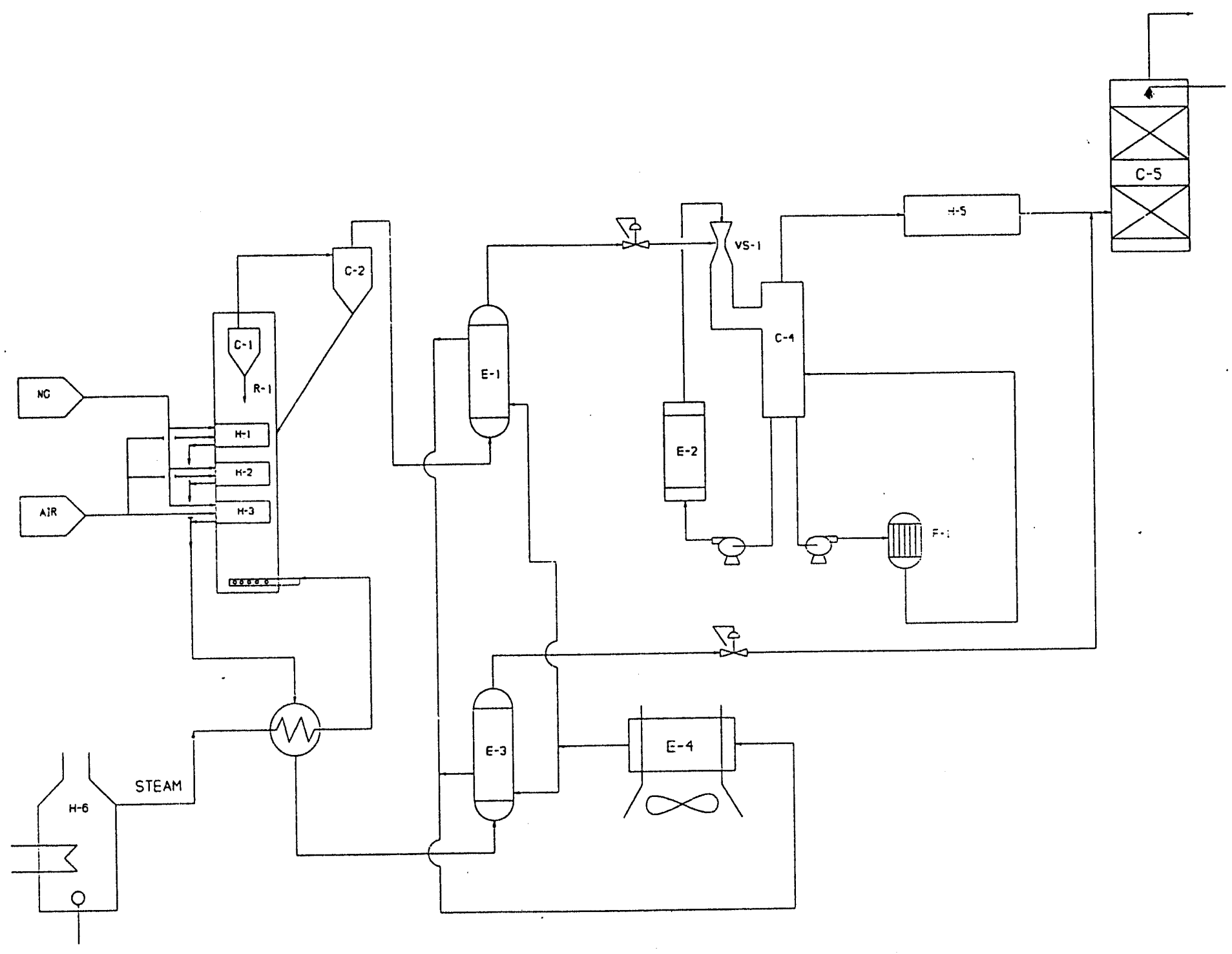

FIGURE 4: MODIFIED PROCESS FLOW DIAGRAM 


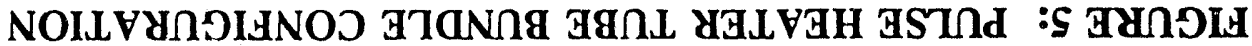

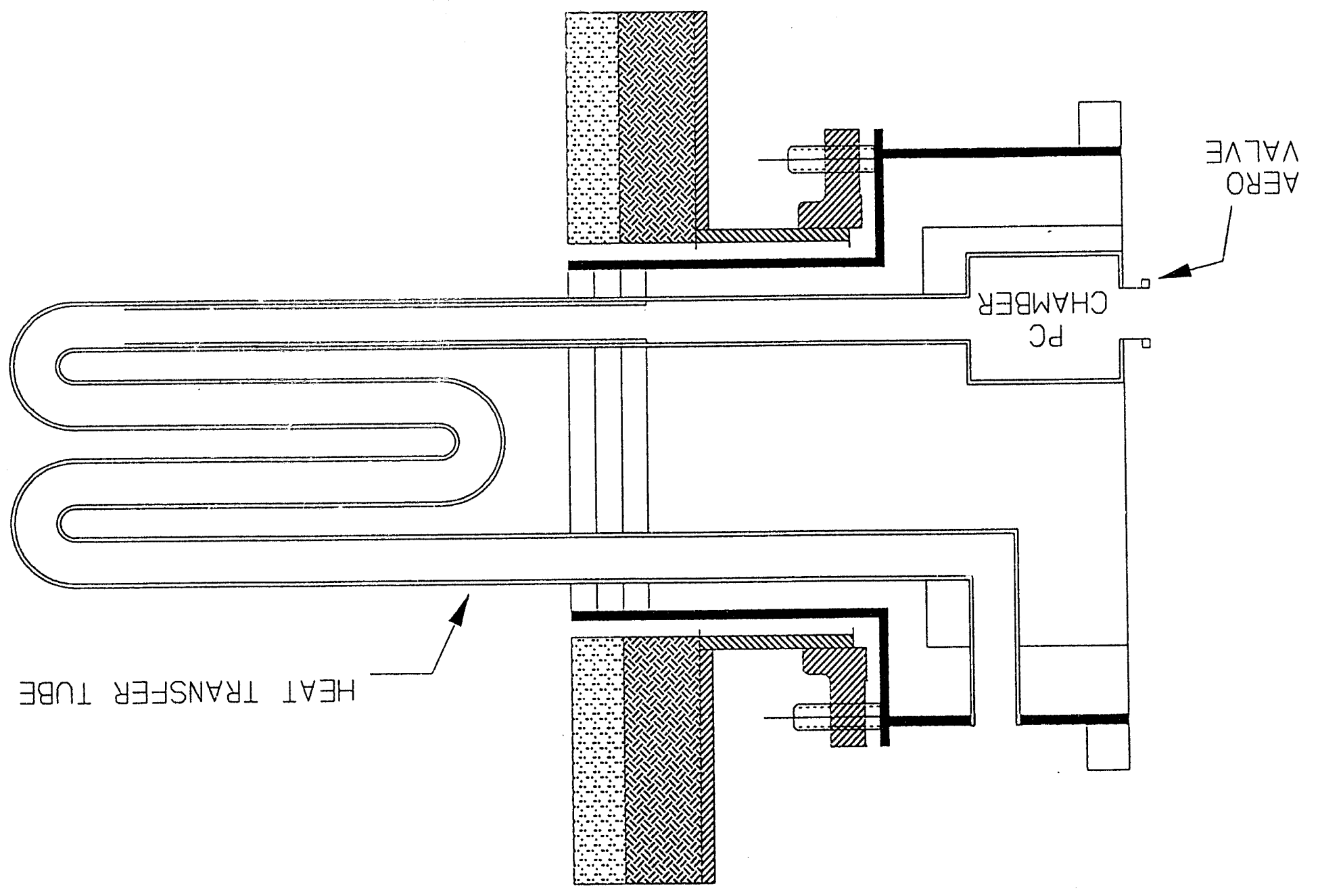




\section{CONCLUSIONS}

Although the project anticipated that the existing system at the MTCI Development Laboratory in Santa Fe Springs, California could be modified with relatively modest cost, analysis of the modifications from a safety and reliability viewpoint mandated that a new reactor vessel and other components should be fabricated to code for the project. This has resulted in an evaluation of the current Statement of Work and estimated cost of the project. MTCI recommendations with respect to modifying both are being prepared for submission to the West Virginia University Project Manager.

The fabrication of the reactor top and bottom sections is 100 percent completed. The pulse heater design is 50 percent complete. The reactor is being made ready for refractory pouring. 


\section{PLANS FOR NEXT REPORTING PERIOD}

During the next period the fabrication of the venturi scrubber, superheater, heat exchanger and filter system will be completed and assembly and installation initiated. 
Microbial Enrichment for Enhancing Biodegration of Hazardous Organic Wastes in Soil (Project MC-18)

\author{
Quarterly Report for Period \\ for Period October 1 through December 31, 1993
}

Work Performed Under Contract

No.: DE-FC21-92MC29467

\author{
For \\ U.S. Department of Energy \\ Office of Fossil Energy \\ Morgantown Energy Technology Center \\ Morgantown, West Virginia
}

By

Alan J. Sexstone, Department of Environmental Microbiology

Chris M. Atkinson, Department of Mechanical and Aerospace Engineering

West Virginia University

Morgantown, West Virginia 
TABLE OF CONTENTS

1.0

EXECUTIVE SUMMARY $\ldots \ldots \ldots \ldots \ldots \ldots \ldots \ldots \ldots$ 


\subsection{EXECUTIVE SUMMARY}

Problem and Research Objectives: The purpose of the current proposed study will be to develop a reliable in vitro assay to directly compare selected commercially available microbial inocula for enhancing biodegradation of mixed organic wastes by soil composting. The products to be initially will be taken from compilations provided by the EPA National Contingency Plan Product Schedule (NCPPS) or the EPRI Advanced Bioremediation Assessment and Technology Evaluation Program (ABATE). While both programs have identified microbial products, neither has directly compared efficacy for inoculation in soil composting. Inocula identified from the lists will be obtained directly from the remediation firms which produce them and used in replicated laboratory experiments to determine treatment effects on the rate and extent of biodegradation and formation of characteristic degradation byproducts. The products will be tested in both a surface and subsurface soil which have been excavated and amended for waste composting. Soils will be contaminated with a model mixed waste containing hydrocarbons, solvents, and polynuclear aromatic hydrocarbons. Biodegradation of the pollutants will be monitored in the presence and absence of inoculation. A specific objective of the work will be to compare the efficacy of bioaugmentation in a surface and subsurface soil. The working hypothesis to be tested is whether positive effects of bioaugmentation are greatest in the subsurface soil due to reduced microbial competition in this environment. Results of the initial one year study will generate specific comparisons of bioaugmentation products in a model test system. Our intention is to develop a more generalized testing procedure and expertise, which we can employ to examine bioaugmentation / biodegradation of a variety of wastes and / or waste sites. During the course of the year we will expand our contacts within the field of bioremediation, making specific expertise in this field available locally to WVU / NRCCE / METC.

Project Status: This is the first quarter of our study. We are currently devoting our efforts to reviewing the literature, identifying an appropriate research technician to assist with the project, and contacting remediation firms to obtain inoculum. Project Director Dr. Ray Lovett has contacted the firm ARCTECH to discuss possible collaborative work. We have contacted researchers at Texas A\&M University who have demonstrated positive effects of commercial inocula in soil to discuss their experience. Funds for the project have recently been put in place so that we now can acquire necessary chemicals and supplies, and set up our analytical procedures. We will concentrate our efforts on perfecting extraction and analysis of the model waste oil during the coming quarter and initiating our bioremediation experiments. 
Soil Decontamination with a Packed Flotation Column (Project MC-19)

\author{
Quarterly Report for Period \\ for Period October 1 through December 31, 1993
}

Work Performed Under Contract

No.: DE-FC21-92MC29467

\author{
For \\ U.S. Department of Energy \\ Office of Fossil Energy \\ Morgantown Energy Technology Center \\ Morgantown, West Virginia
}

\author{
By \\ Eung Ha Cho, Ph.D. \\ Felicia F. Peng, Ph.D. \\ David C. Yang, Ph.D. \\ Department of Mineral Processing Engineering \\ West Virginia University \\ Morgantown, West Virginia
}

February 1994 


\begin{abstract}
The general objective of this research is to evaluate a new technology for remediation of plutonium contaminated soil at the Nevada Test Site. The major effort of the research will be to optimize a flotation process in which a packed column is used to float fine particles of cerium oxide that will be selected as a surrogate because of their similarities in flotation chemistry.

Laboratory tests will be carried out with a Hallimond flotation cell to determine the best flotation reagent scheme. Laboratory tests will be conducted with a $3^{\prime \prime}$ packed column aiming at identifying key factors that will affect the operation of the process. The reagent scheme determined with a Hallimond cell will be utilized in these tests. The results of the tests will be analyzed to determine a set of operating variables with which the most effective separation can be achieved.
\end{abstract}

A team of one graduate research assistant, a P.I. and two Co-P.I.'s have been formed for this research. One of the Co-P.I.'s is Dr. David C. Yang, the inventor of the packed flotation column. He will help control the performance of the packed flotation column and thus will direct this research towards success. We are currently directing our efforts in setting up a 3 " packed column and finding an effective analytical method for cerium. 
TABLE OF CONTENTS

$1.0 \quad$ EXECUTIVE SUMMARY $\ldots \ldots \ldots \ldots \ldots \ldots \ldots \ldots \ldots \ldots \ldots$

ii 


\subsection{EXECUTIVE SUMMARY}

The major objective of this research is to evaluate a new technology for remediation of plutonium contaminated soil at the Nevada Test Site. The major effort of the research will be to optimize a flotation process in which a packed column is used to float fine particles of cerium oxide that will be selected as a surrogate because of their similarities in flotation chemistry.

A similar project is underway elsewhere using a Campbell centrifugal jig and an air-sparged hydrocyclone. However, there is a strong indication in the literature that the packed flotation column can outperform those devices in separating fine mineral particles. This is because the packed column has some excellent features in its operation. The main feature is that casual attachment between a non-floatable particle and air bubbles will be minimized due to repeated foam scrubbing against the packing elements inside the column. This is the basis upon which the research is justified.

Laboratory tests will be carried out with a Hallimond flotation cell to determine the best flotation reagent scheme. Laboratory tests will be conducted with a $3^{\prime \prime}$ packed column aiming at identifying key factors that will affect the operation of the process. The reagent scheme determined with a Hallimond cell will be utilized in these tests. The results of the tests will be analyzed to determine a set of operating variables with which the most effective separation can be achieved.

A team of one graduate research assistant, a P.I. and two Co-P.I.'s have been formed for this research. One of the Co-P.I.'s is Dr. David C. Yang, the inventor of the packed flotation column. He will help control the performance of the packed flotation column and thus will direct this research towards success. We are currently directing our efforts in setting up a 3" packed column and finding an effective analytical method for cerium. 
Treatment of Volatile Organic Compounds (VOCs) Using Biofilters (Project MC-20)

\author{
Quarterly Report for Period \\ for Period October 1 through December 31, 1993
}

Work Performed Under Contract

No.: DE-FC21-92MC29467

\author{
For \\ U.S. Department of Energy \\ Office of Fossil Energy \\ Morgantown Energy Technology Center \\ Morgantown, West Virginia
}

\begin{abstract}
By
Shahab D. Mohaghegh, Department of Petroleum \& Natural Gas Engineering

Patrick E. Carriere, Department of Civil Engineering

West Virginia University

Morgantown, West Virginia
\end{abstract}

February 1994 


\begin{abstract}
The principal objective of the current research is to determine the removal efficiency of volatile organic compounds using innovative biofilters. The risk posed by volatile organic compounds to public health due to their mobility in all the three sinks of pollutants i.e. air, soil and water prompts this research group to obtain the essential technical details, which otherwise are meager in literature. The removal efficiency of these contaminants will be studied and compared with those obtained by existing technologies.
\end{abstract}

A Gas Chromatograph will be used to analyze the highly precise data. 
TABLE OF CONTENTS

1.0 EXECUTIVE SUMMARY $\ldots \ldots \ldots \ldots \ldots \ldots \ldots \ldots$ 


\subsection{EXECUTIVE SUMMARY}

The principal objective of this research is to investigate the removal efficiency of Volatile Organic Compounds (VOCs) using biofilters. The VOCs are known to be potentially hazardous to public health, which necessitates this research. The Biofiltration is an innovative Air Pollution control technology in which off gases containing biodegradable VOCs are passed through a biologically activated material. The results obtained in terms of removal efficiency and economics will be compared with those of conventional technologies.

We have carried out inventory search to find out the possibility of utilizing the available analytical instruments (GC). A Varian make GC (Model\#3410), was identified to suit our requirement which further requires a $\mathrm{PC}$, a suitable column and updating of the available software. We are currently in the process of procurement of the above requirements. We have contacted various commercial suppliers of equipment for research and experimental work. The detailed engineering drawing (following Figure) of the experimental setup is sent and we are awaiting their quotations. The literature has been reviewed to identify the most appropriate microorganisms for our current research study.

Our research team consisting of two Graduate students, is currently working on reviewing literature, the detailed engineering design of experimental setup and in the process of procuring necessary analytical instruments, equipment and chemicals.

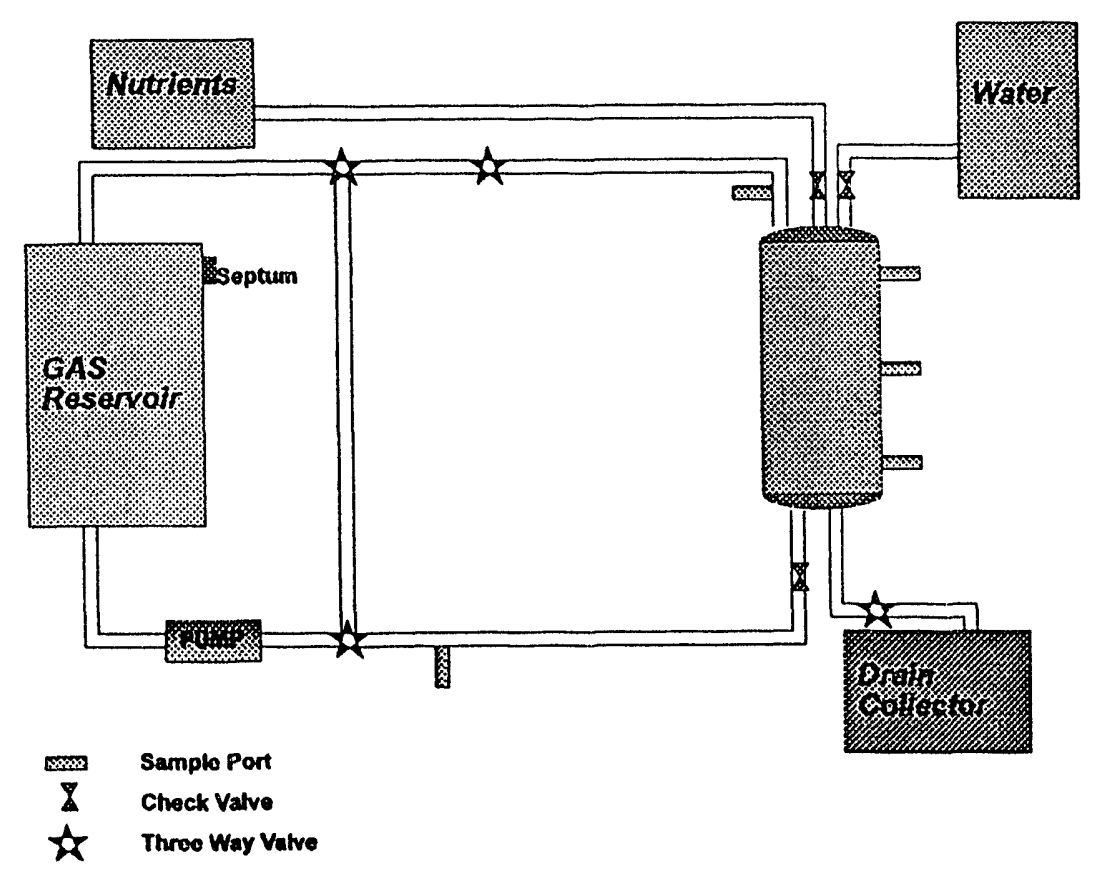


Use of Granular Activated Carbon Columns for the Simultaneous Removal of Organic, Heavy Metals, and Radionuclides

(Project MC-21)

\author{
Quarterly Report for Period \\ for Period October 1 through December 31, 1993
}

Work Performed Under Contract

No.: DE-FC21-92MC29467

For

U.S. Department of Energy

Office of Fossil Energy

Morgantown Energy Technology Center

Morgantown, West Virginia

By

Brian E. Reed, Ph.D.

Patrick E. Carriere, Ph.D.

Department of Civil Engineering

West Virginia University

Morgantown, West Virginia

February 1994 


\begin{abstract}
The goal of this research is to investigate the feasibility (technical and economic) of using granular activated carbon (GAC) columns to treat an aqueous waste stream containing organic contaminants, heavy metals, and radionuclides. If the GAC column process is demonstrated to be technically feasible and cost effective, it will represent a significant tool in the plan to remediate the nation's hazardous waste sites. Specific objectives of the research, signified as Tasks 1 through 3, are to investigate the following topics:
\end{abstract}

Task 1: The use of GAC columns to treat a waste containing organic compounds and heavy metals.

Task 2: The efficacy of using activated carbon to remove radionuclides.

Task 3: The use of GAC columns to treat a mixed-waste.

Norit Americas Inc. (formerly American Norit Co., Inc.), an activated carbon manufacturer, is the private sector partner onhis research. The following tasks have or are being conducted as of the writing of this report:

1. Three graduate students (1 Ph.D., 2 M.S.) have been hired.

2. Literature search on the use of GAC columns for aqueous waster treatment (on-going).

3. Development of analytical methods to measure the organic and inorganic contaminants (ongoing).

4. Set-up of the pilot-scale GAC column system that was delivered by Norit Americas, Inc. (completed).

5. Initial testing of the pilot-scale GAC column system using $1 \mathrm{mg} / \mathrm{L} \mathrm{Pb}$ (on-going). 


\section{TABLE OF CONTENTS}

Section No.

Page No.

1. EXECUTIVE SUMMARY 


\section{EXECUTIVE SUMMARY}

The goal of this research is to investigate the feasibility (technical and economic) of using granular activated carbon (GAC) columns to treat an aqueous waste stream containing organic contaminants, heavy metals, and radionuclides. If the GAC column process is demonstrated to be technically feasible and cost effective, it will represent a significa.: tool in the plan to remediate the nation's hazardous waste sites. Specific objectives of the research, signified as Tasks 1 through 3 , are to investigate the following topics:

Task 1: The use of GAC columns to treat a waste containing organic compounds and heavy metals.

Task 2: The efficacy of using activated carbon to remove radionuclides.

Task 3: The use of GAC columns to treat a mixer-waste.

Norit Americas Inc. (formerly American Norit Co., Inc.), an activated carbon manufacturer, is the private sector partner on this research. The following tasks have or are being conducted as of the writing of this report:

1. Three graduate students (1 Ph.D., 2 M.S.) have been hired.

2. Literature search on the use of GAC columns for aqueous waster treatment (on-going).

3. Development of analytical methods to measure the organic and inorganic contaminants (ongoing).

4. Set-up of the pilot-scale GAC column system that was delivered by Norit Americas, Inc. (completed).

5. Initial testing of the pilot-scale GAC column system using $1 \mathrm{mg} / \mathrm{L} \mathrm{Pb}$ (on-going). 
Compact Mercuric Iodide Detector Technology Development (Project MC-22)

Quarterly Report for Period

for Period October 1 through December 31, 1993

Work Performed Under Contract

No.: DE-FC21-92MC29467

\author{
For \\ U.S. Department of Energy \\ Office of Fossil Energy \\ Morgantown Energy Technology Center \\ Morgantown, West Virginia
}

\author{
By \\ Donald W. Lyons, Ph.D. \\ Gora C. Nandi, Ph.D. \\ Department of Mechanical and Aerospace Engineering \\ West Virginia University \\ Morgantown, West Virginia
}




\begin{abstract}
The general objective of this research is to explore the possibility of using the Mercuric lodide as a radiation detector, based on the already established characteristics of the compound. A thorough literature search has been done. One graduate student has already joined the research team. Since no Mercuric Iodide crystal is commercially available it is planned to prepare them. A development program will be undertaken to grow the crystal starting with Mercuric Iodide powder. It is expected that several months will be required to develop the technology and grow the crystals. In order to allow initial experimentation with Mercuric Iodide to begin, pallets of compressed powder will be prepared in the first months. Initial experimentation to study radiation detector characterstics will be undertaken using the pallets simultaneously with the work activity to grow crystals.
\end{abstract}

So far more than 15 commercial vendors who are involved in supplying materials in the related field had been contacted. One of them, Alpha-Aesar has been selected for placing an order.Their product seems to satisfy our purity requirement within a reasonable price range.

\title{
The future research plan includes:
}

1) Growing Crystal.

2) Making Pallets

3) Studying the effect of the concentration of etching solution Potassium Iodide and etching rates and recommending the concentration to achive the best detector.

4) Studying the effect of Potassium Iodide impurities on the behavior of the detector.

5) Evaluating an alternate procedure for developing electrodes to avoid diffusion of electrode vapors inside the crystal. 


\section{TABLE OF CONTENTS}

$1.0 \quad$ Executive Summary $\ldots \ldots \ldots \ldots \ldots \ldots \ldots \ldots$ 


\subsection{Executive Summary}

The objective of this research is to explore the possibility of developing the Mercuric lodide $\left(\mathrm{HgI}_{2}\right)$ technology to produce an improved radiation detector. The approach is based on previously established use of $\mathrm{HgI}_{2}$ for the detection of gamma- rays. We will start by procuring $\mathrm{HgI}_{2}$ powder and growing crystal and forming $\mathrm{HgI}_{2}$ pallet. The feasibility of using pallet for gamma - ray detection will be studied by carrying out the experiments with crystal as well as pallet and then comparing the results.

So far we have contacted 15 commercial vendors for $\mathrm{HgI}_{2}$ powder. The product from Alpha Aesar fits our requirements regarding purity with in a reasonable price range. $150 \mathrm{gms}$ of $\mathrm{HgI}_{2}$ with two grade purities had been ordered. Since it is highly poisonous some precautionary measures must be taken before handling during experimentation. Preparations are underway to procure the facilities and supplies necessary for safe handling.

A graduate student has joined the research team. A thorough literature search has been coompleted. The process of gathering chemicals, supplies and setting up of experimentation has already been started.

\section{Our future plans are to:}

- Accomplish crystal growing and pallet preparation

- Study the effect of varying the concentration of etching solution KI and etching rates and to recommend the best combination of the two.

- Study the effect of various impurities which are present in KI on the behavior of detector.

- Search for an alternate etching solution if possible.

- Study the effects of using various materials as contacts.

- Evaluate alternate procedure for developing electrodes to avoid the diffusion of electrode vapors in to the crystal.

- Develop recommendations to avoid polarization. 
Evaluation of IR and Mass Spectrometric Techniques for On-Site Monitoring of Volatile Organic Compounds

(Project MC-23)

Quarterly Report for Period

for Period October 1 through December 31, 1993

Work Performed Under Contract

No.: DE-FC21-92MC29467

For

U.S. Department of Energy

Office of Fossil Energy

Morgantown Energy Technology Center

Morgantown, West Virginia

By

Mohindar S. Seehra, Department of Physics

Fred L. King, Department of Chemistry

West Virginia University

Morgantown, West Virginia

February 1994 


\section{TABLE OF CONTENTS}

$1.0 \quad$ EXECUTIVE SUMMARY $\ldots \ldots \ldots \ldots \ldots \ldots \ldots \ldots \ldots \ldots$ 


\subsection{EXECUTIVE SUMMARY}

The objective of this project is the identification of optimal instrumental approaches to the field determination of Volatile Organic Compounds, such as chlorinated hydrocarbons and aromatics. IR and Mass Spectrometries were selected for evaluation because they are currently the strongest techniques available for this purpose. Professor Seehra will focus on a critical evaluation of the IR techniques based upon literature research, telephone conferences, and when necessary, site visits. Professor King will evaluate tandem mass spectrometry methods to be implemented with an ion trap mass spectrometry system for the characterization of a series of selected target compounds.

During the 1994 Spring Semester, one Physics graduate student will carry out a detailed survey of the literature regarding field IR spectrometry methods. Basic questions regarding: the relative merits of point vs. open path FT-IR sampling, various baseline correction methods, compound spectral fingerprints for unambiguous identification, and portability are under investigation. During the 1994 Summer Semester, Professor Seehra will carry out a critical evaluation of the literature. This will include telephone conferences with the principal researchers in the subject area and, site visits, if needed. This aspect of the project will be completed by Dec. 1994.

In the mass spectrometry project the effort to date has focussed on the acquisition of a suitable ion trap mass spectrometry system and a preliminary search of literature methods for the characterization of target compounds. The focus is on tandem mass spectrometry methods that will eliminate the need for chromatographic separation and therefore reduce the overall size and complexity of the system. A Chemistry graduate student will begin work on the project in the late spring to early summer, once the equipment is in place. By that time, proposed tandem mass spectrometry methods for the quantitative identification of components in a BTEX mixture will be ready for evaluation. Both existing literature methods and newly developed methods will be compared for sensitivity, accuracy, and precision. 
Improved Socio-Economic Assessment of Alternative Environmental Restoration Techniques

(Project MC-24)

\author{
Quarterly Report for Period \\ for Period October 1 through December 31, 1993
}

Work Performed Under Contract

No.: DE-FC21-92MC29467

\author{
For \\ U.S. Department of Energy \\ Office of Fossil Energy \\ Morgantown Energy Technology Center \\ Morgantown, West Virginia
}

\begin{abstract}
By
Andrew M. Isserman, Regional Research Institute

Jerald J. Fletcher, Department of Resource Management

William N. Trumbull, Department of Business and Economics

West Virginia University

Morgantown, West Virginia
\end{abstract}

February 1994 


\section{TABLE OF CONTENTS}

$1.0 \quad$ EXECUTIVE SUMMARY $\ldots \ldots \ldots \ldots \ldots \ldots \ldots \ldots \ldots \ldots \ldots \ldots$ 


\subsection{EXECUTIVE SUMMARY}

Product 1. A critical evaluation of the state of the art of assessment studies.

We are collecting and analyzing copies of assessment studies carried out by a variety of responsible parties, their consultants, and public agencies. To build our collection, a graduate student has made numerous phone calls and has arranged to visit the EPA to review and copy selected documents on file there. The next step is to evaluate these studies, paying particular attention to:

how (and whether) they incorporate issues likely to be of concern to citizens, how they assign weights to those concerns relative to other decision factors, and whether they discuss the citizen concerns in ways that are likely to be convincing to citizens.

Then we shall write a survey article that points out the shortcomings of present methodologies and outlines the properties of a better system. No such study presently exists, at least to the best of our knowledge and that of the people we have contacted.

Product 2. A report summarizing primary citizen concerns and describing ways that they ought to be incorporated correctly into assessment studies.

We shall use the prior studies and our calls to citizen organizations and public agencies to create a list of the main types of citizen concerns, e.g., health risks from incineration. We shall identify the ideal way and alternative second-best feasible ways for incorporating those concerns into a socio-economic assessment study. Toward that end, we are collecting the most recent, relevant economic literature. It focuses on valuing environmental damage, not restoration. We shall determine how each major concern should be valued consistent with economic theory and the information that would be necessary for doing so. Then we shall write an article on this research for journal publication.

Product 3. A report outlining how an improved socio-economic assessment could be carried out for the Winfield sit 2 or another site to be selected by NRCCE/METC.

We shall describe the data needs and, in collaboration with others, feasible strategies for collecting the data. We shall design and explain an interactive assessment methodology. The challenge is to build in citizen participation in a meaningful way that is likely to build consensus for a restoration technology. 

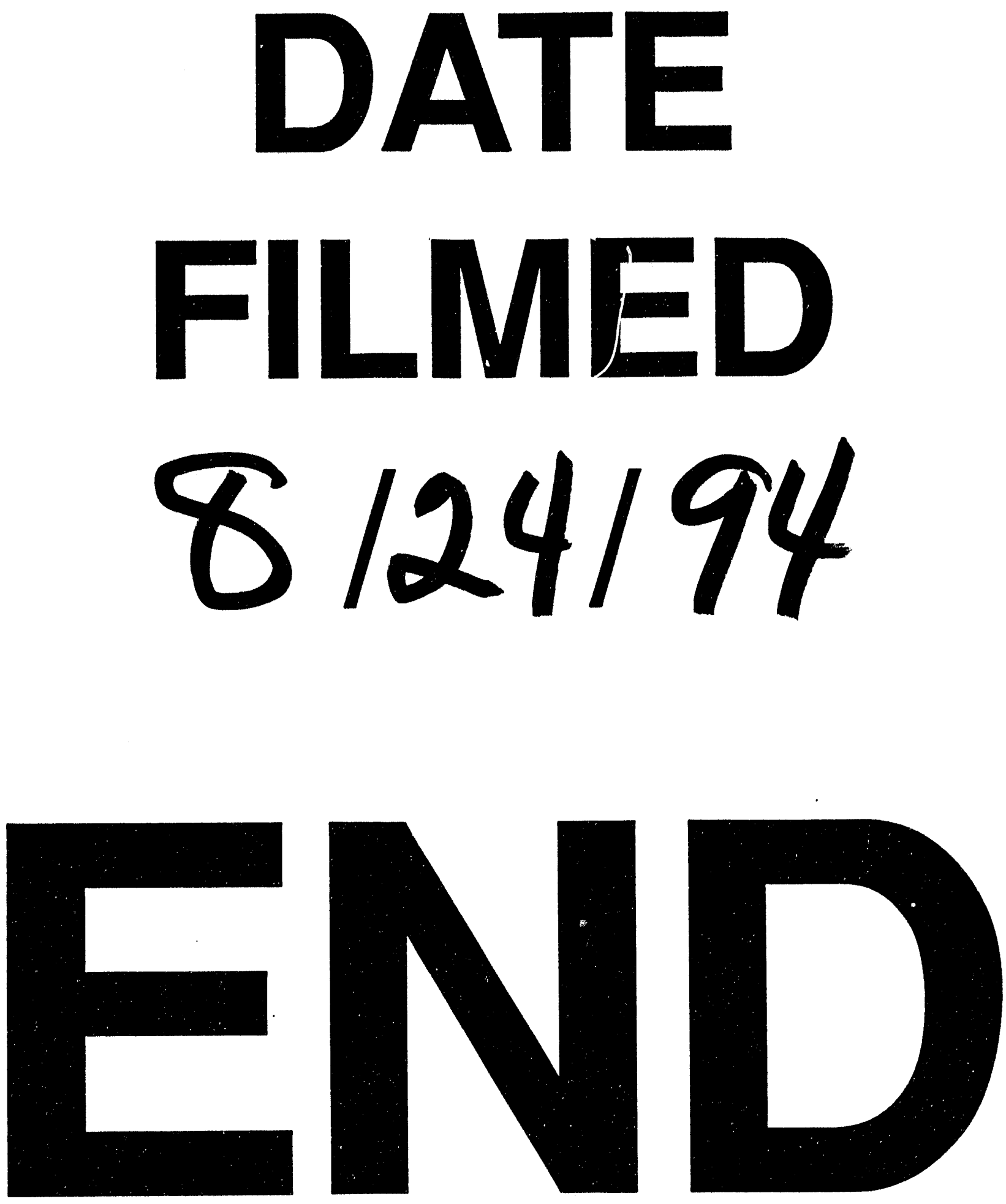


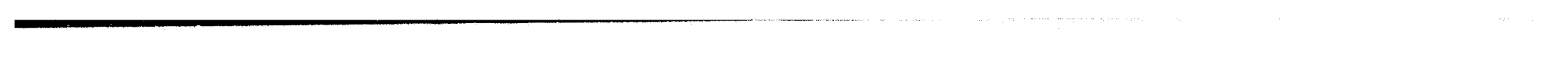$$
\text { . }
$$

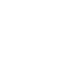$$
\text { - }
$$

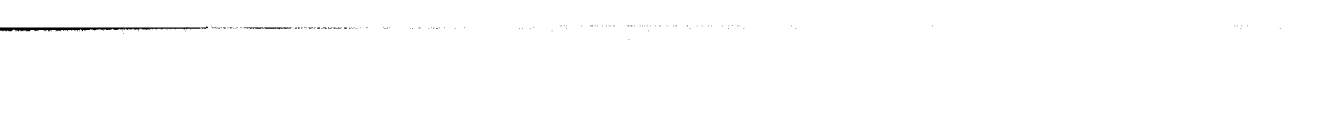

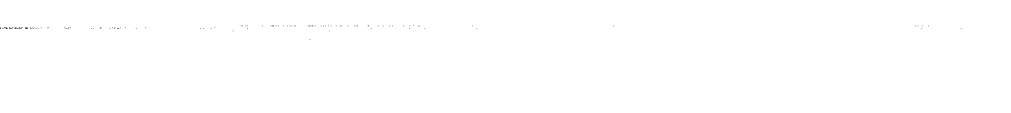

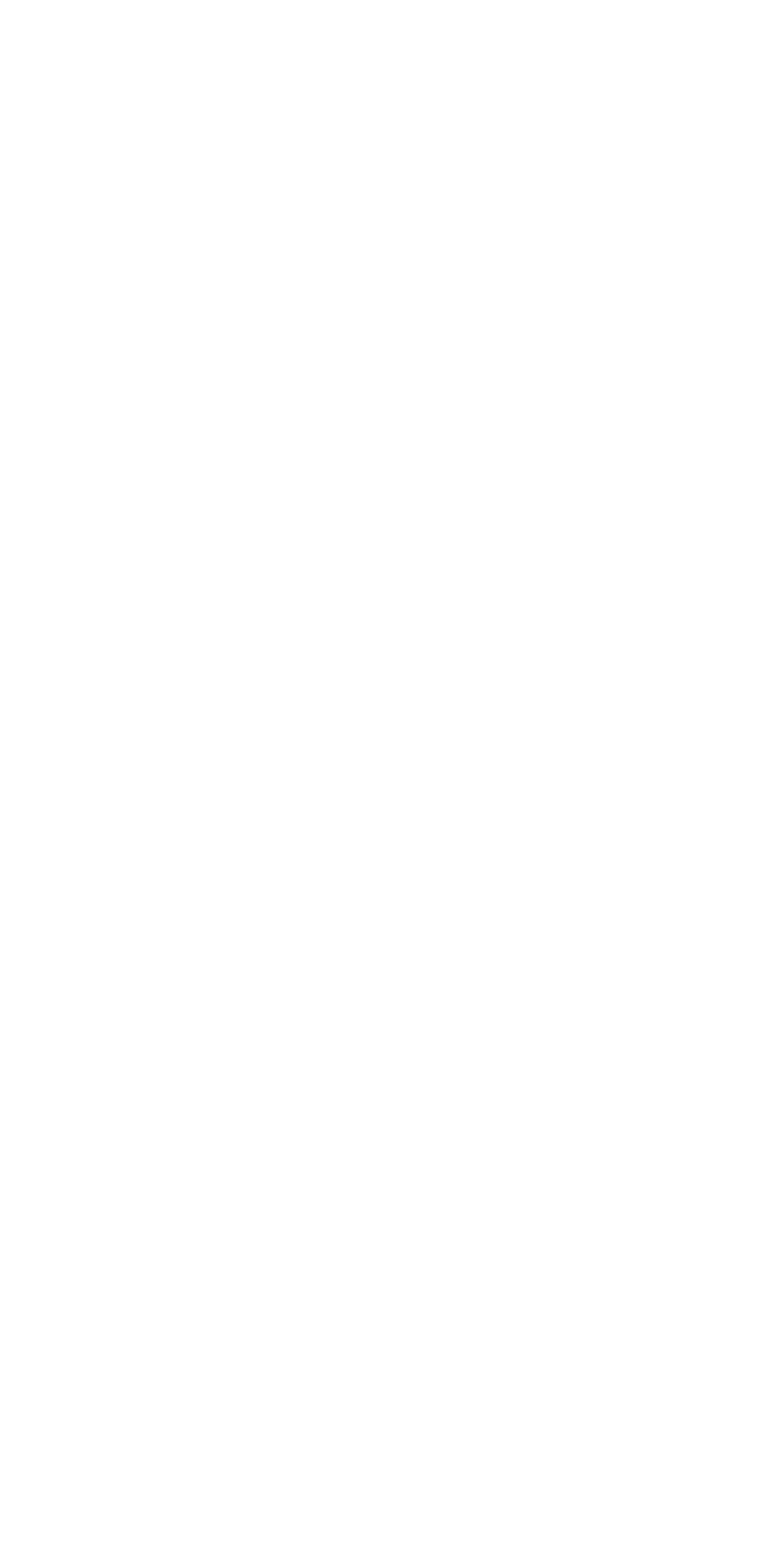

